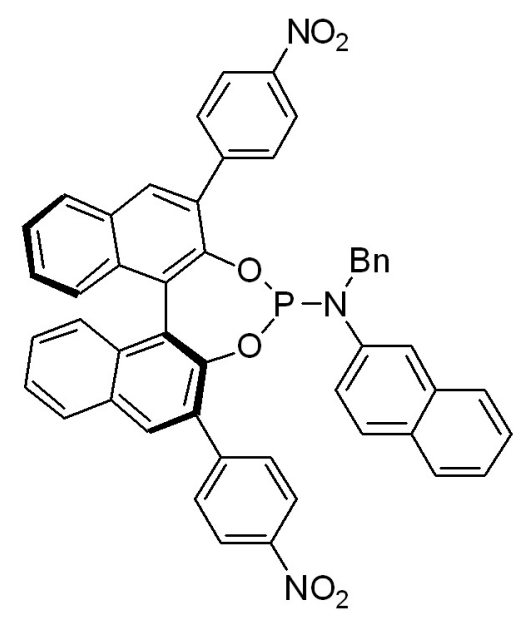

L2

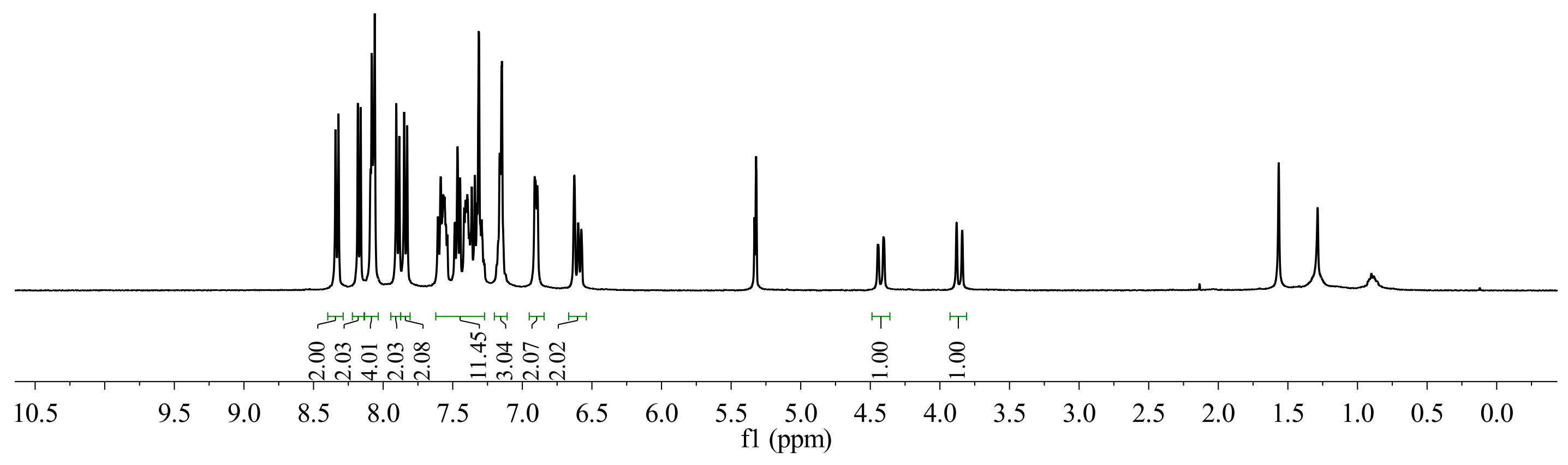




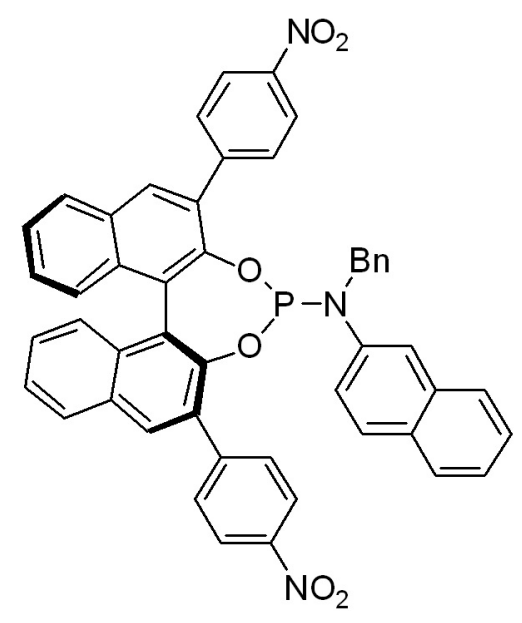

L2

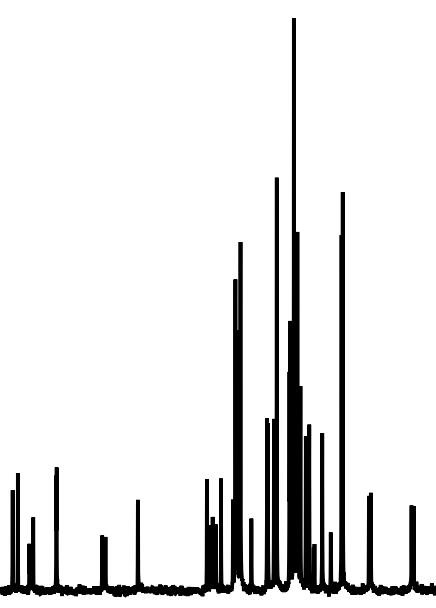

190

180

170

160

150

130

120

110

10090

80

70

60

f1 (ppm) 


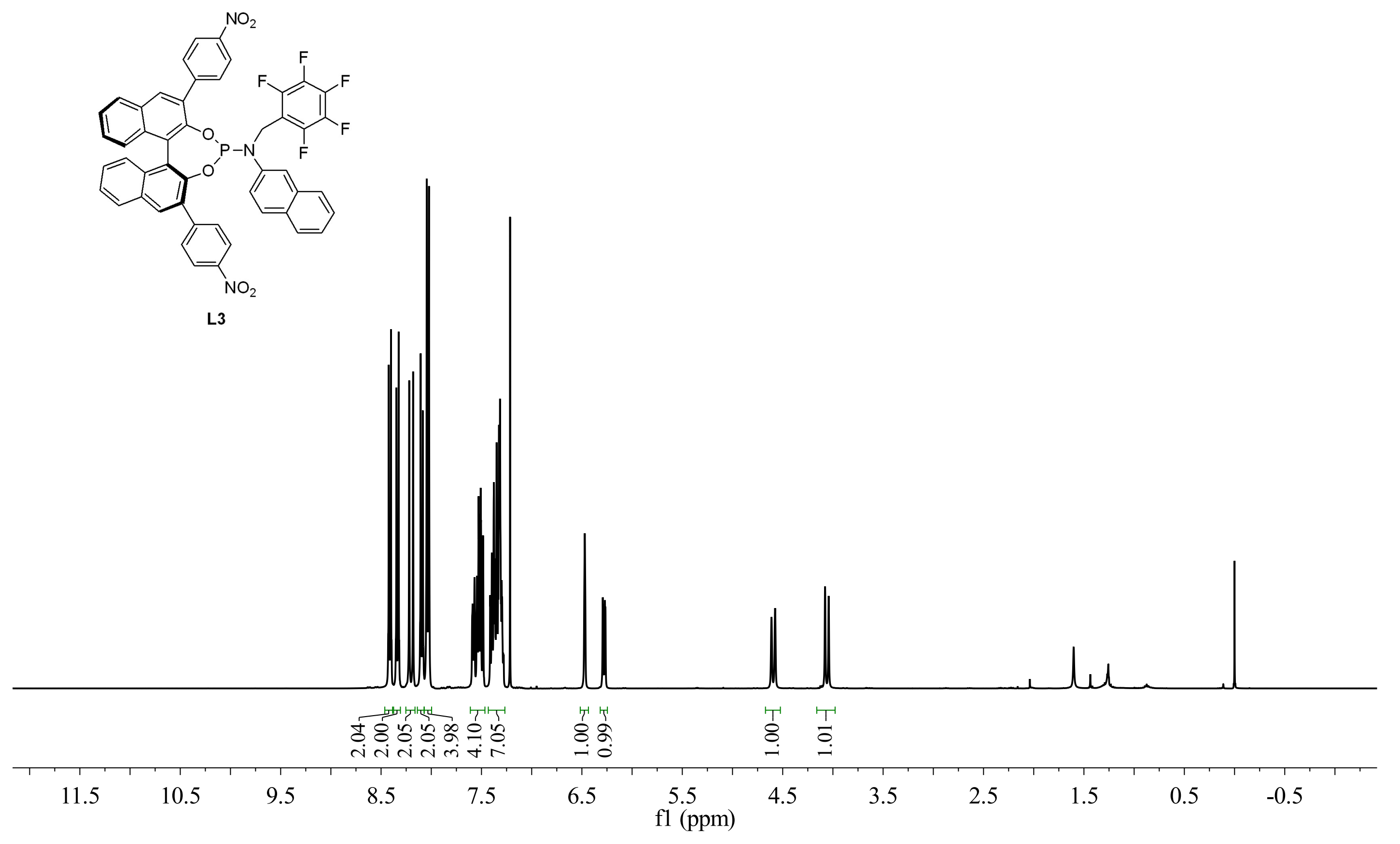




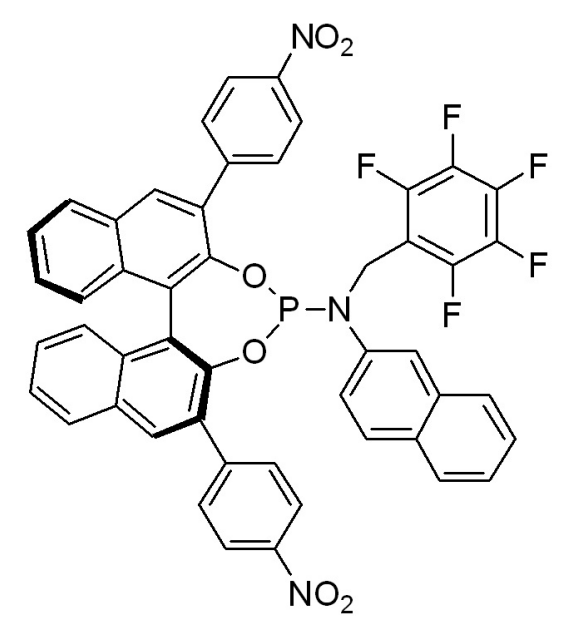

L3

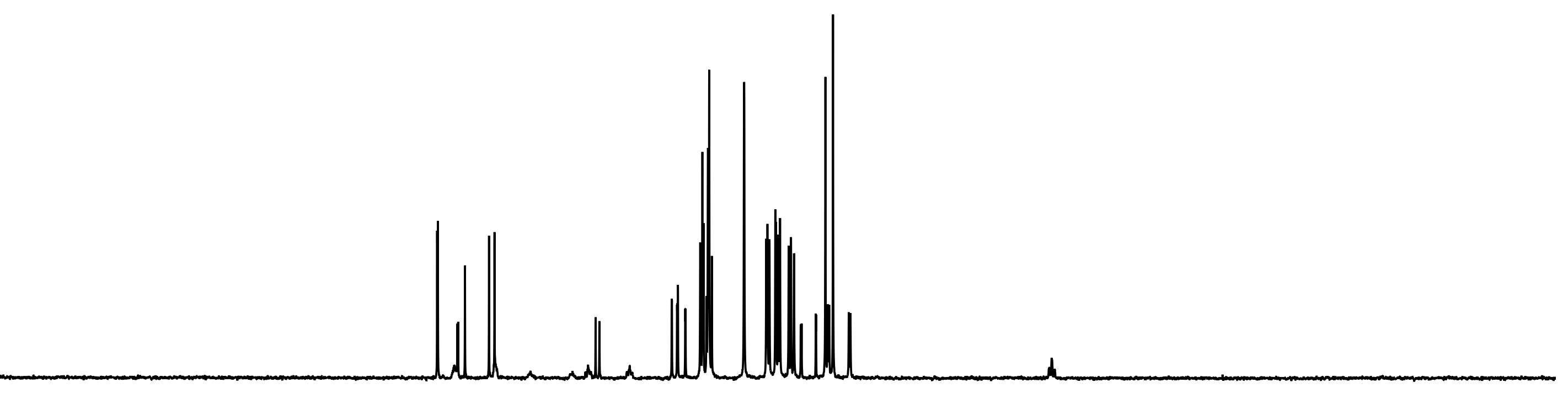




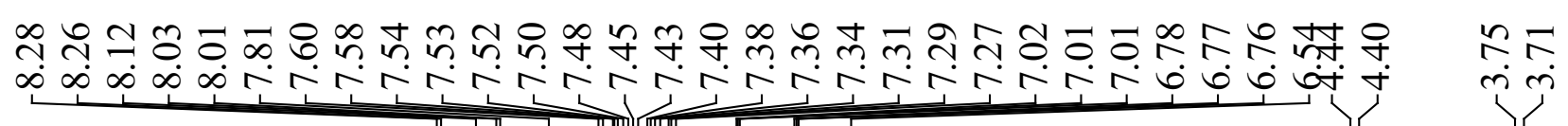
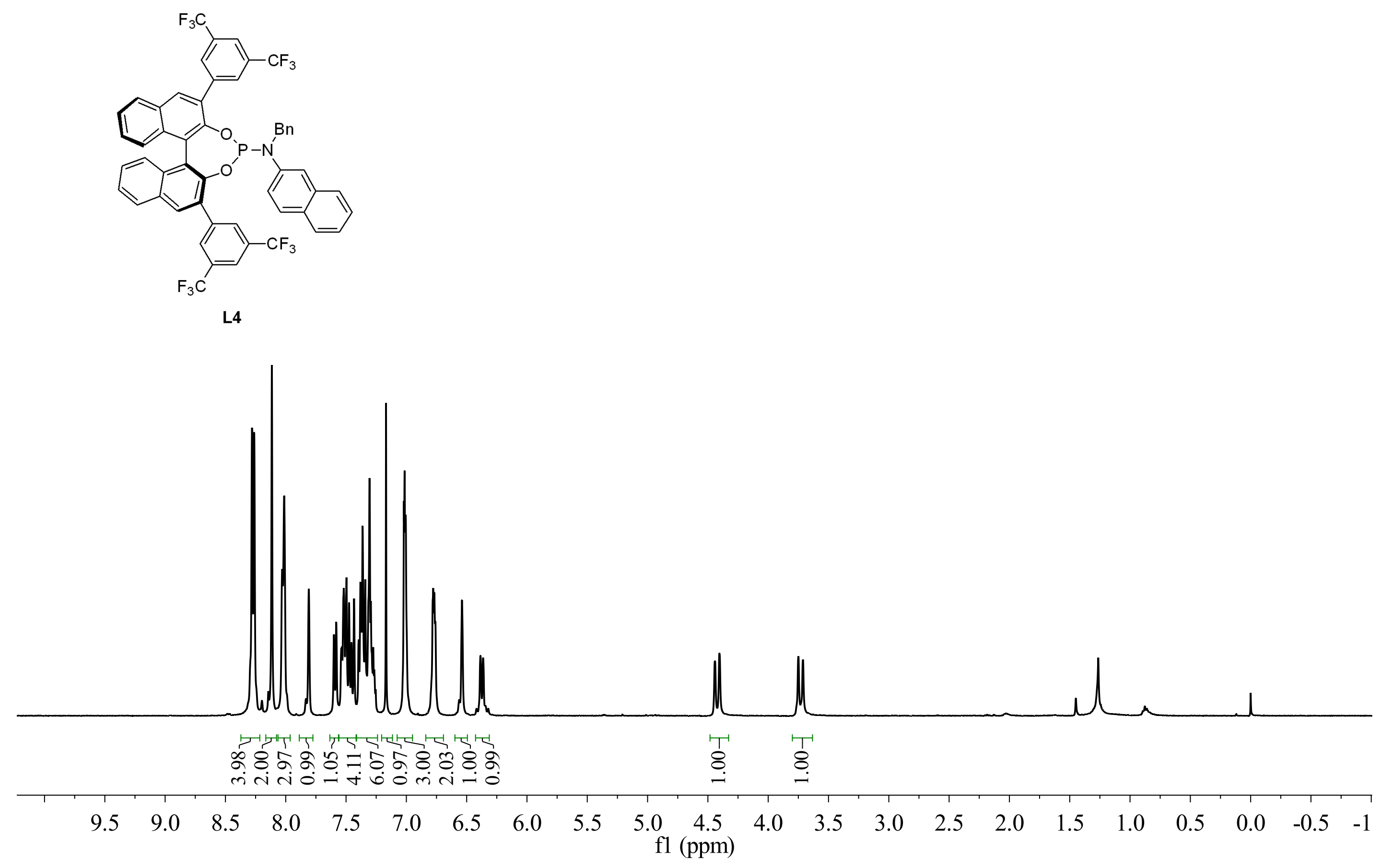


$$
4
$$





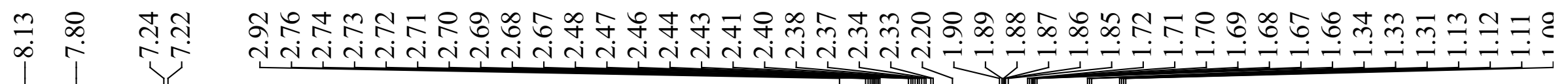

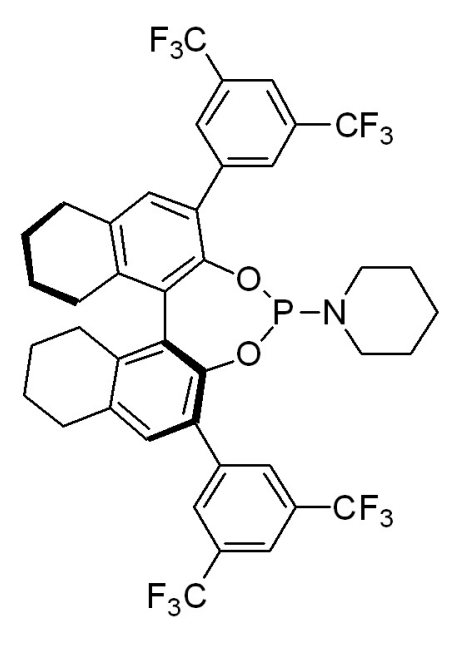

L6

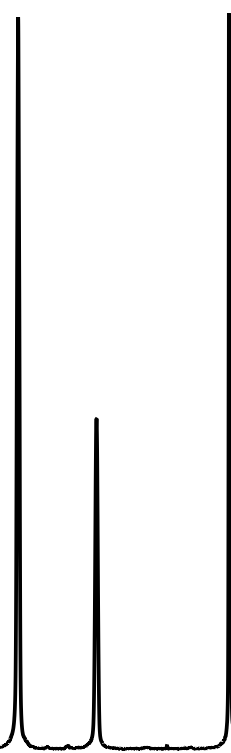

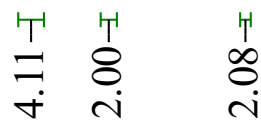

समस पात ?ा?

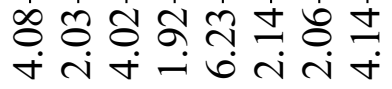

9.5

$\begin{array}{llll}9.0 & 8.5 & 8.0 & 7.5\end{array}$

$\begin{array}{ll}7.0 & 6.5\end{array}$

$6.0 \quad 5.5$

5.0

$\begin{array}{lll}4.5 & 4.0 & 3.5\end{array}$

fl (ppm)
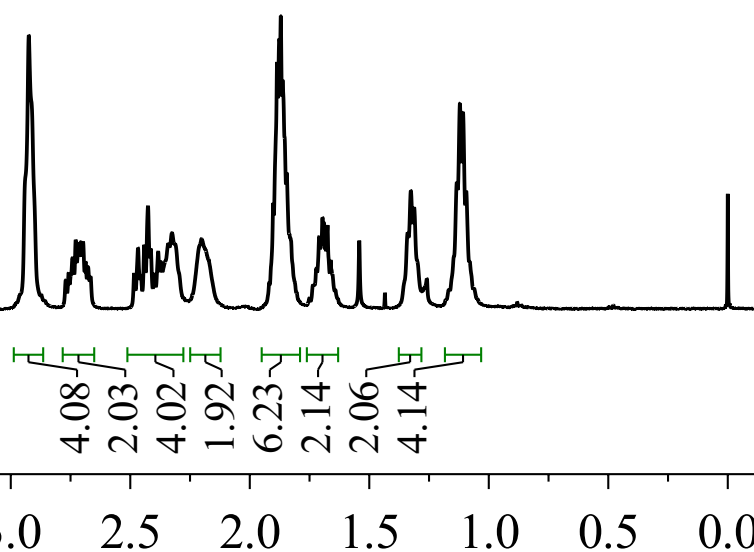

1.5

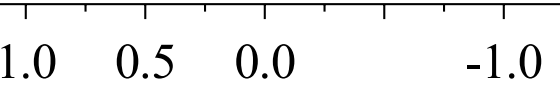




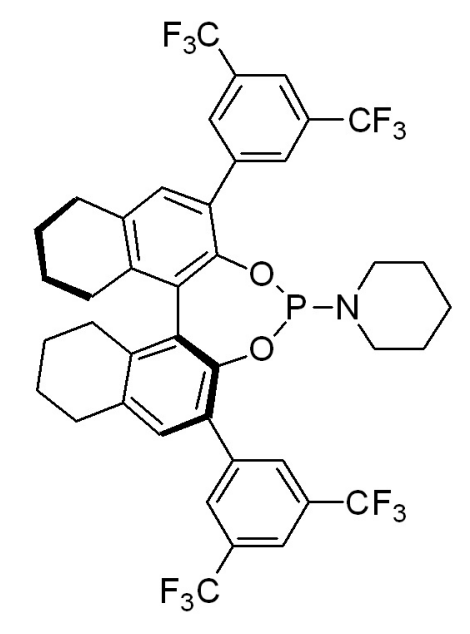

L6

$\begin{array}{lllllllll}80 & 70 & 60 & 50 & 40 & 30 & 20 & 10 & 0\end{array}$




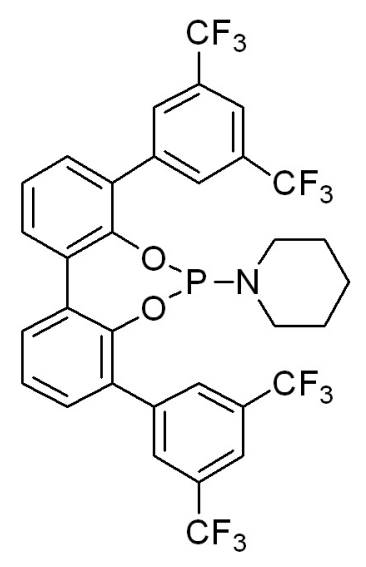

L7

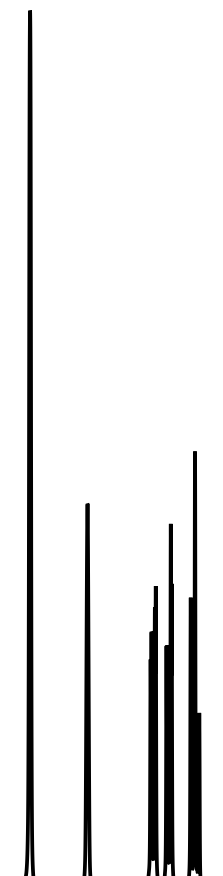




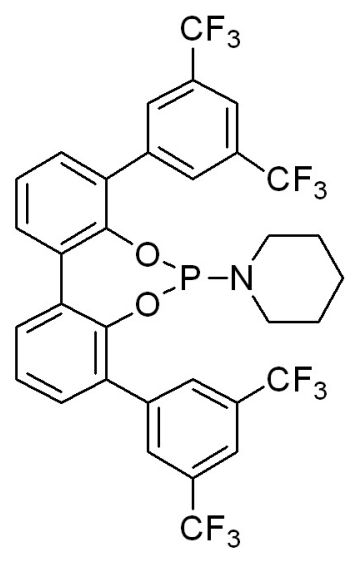

L7

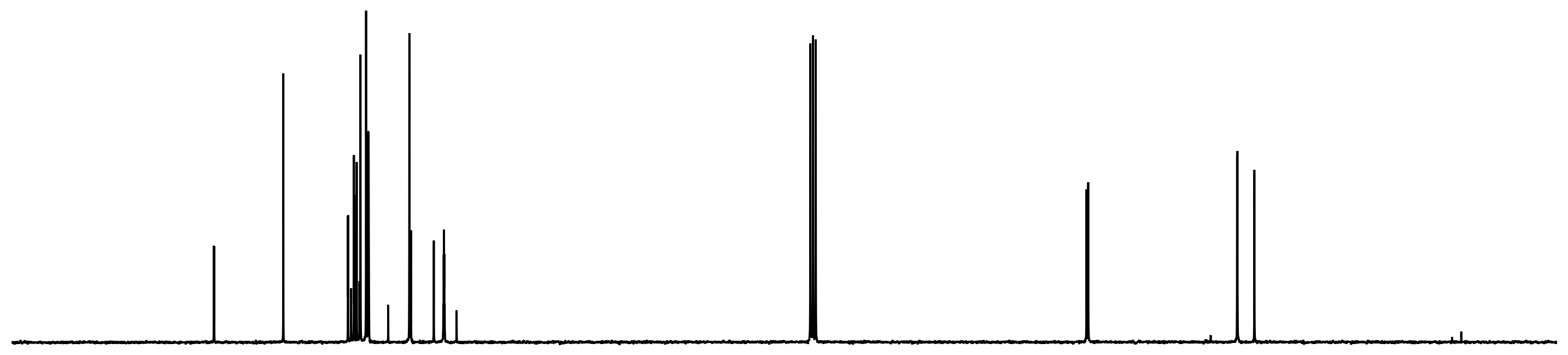



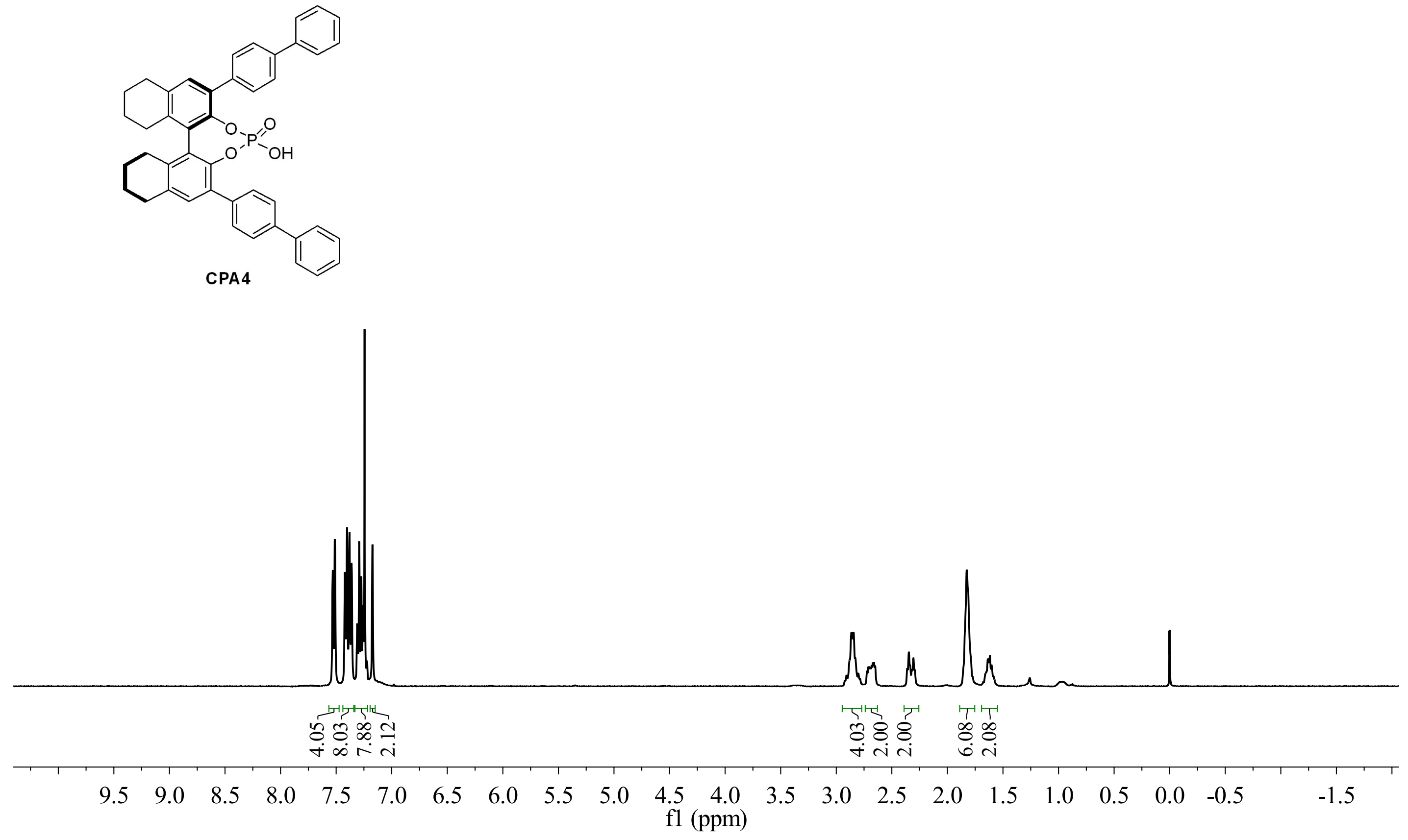


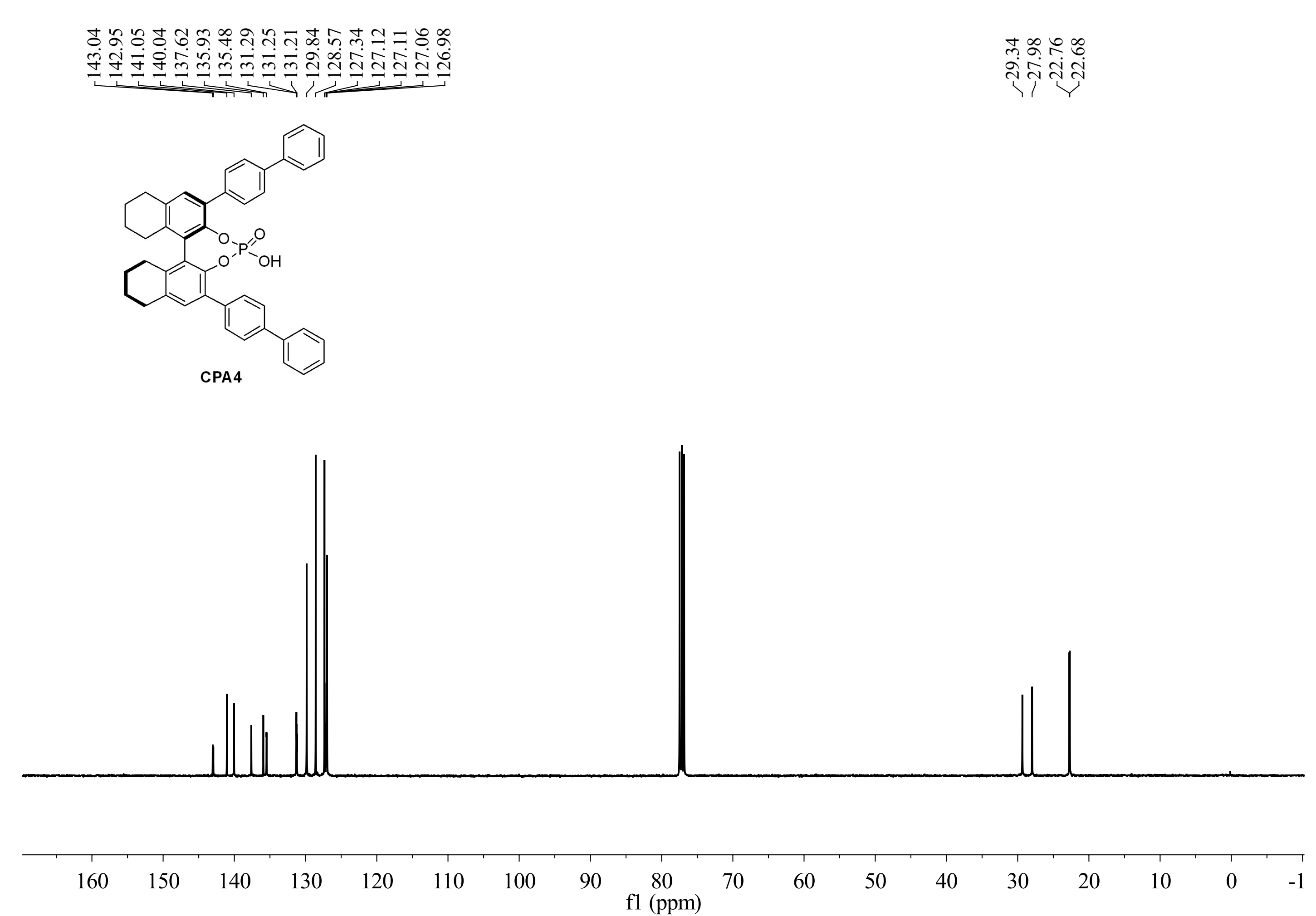



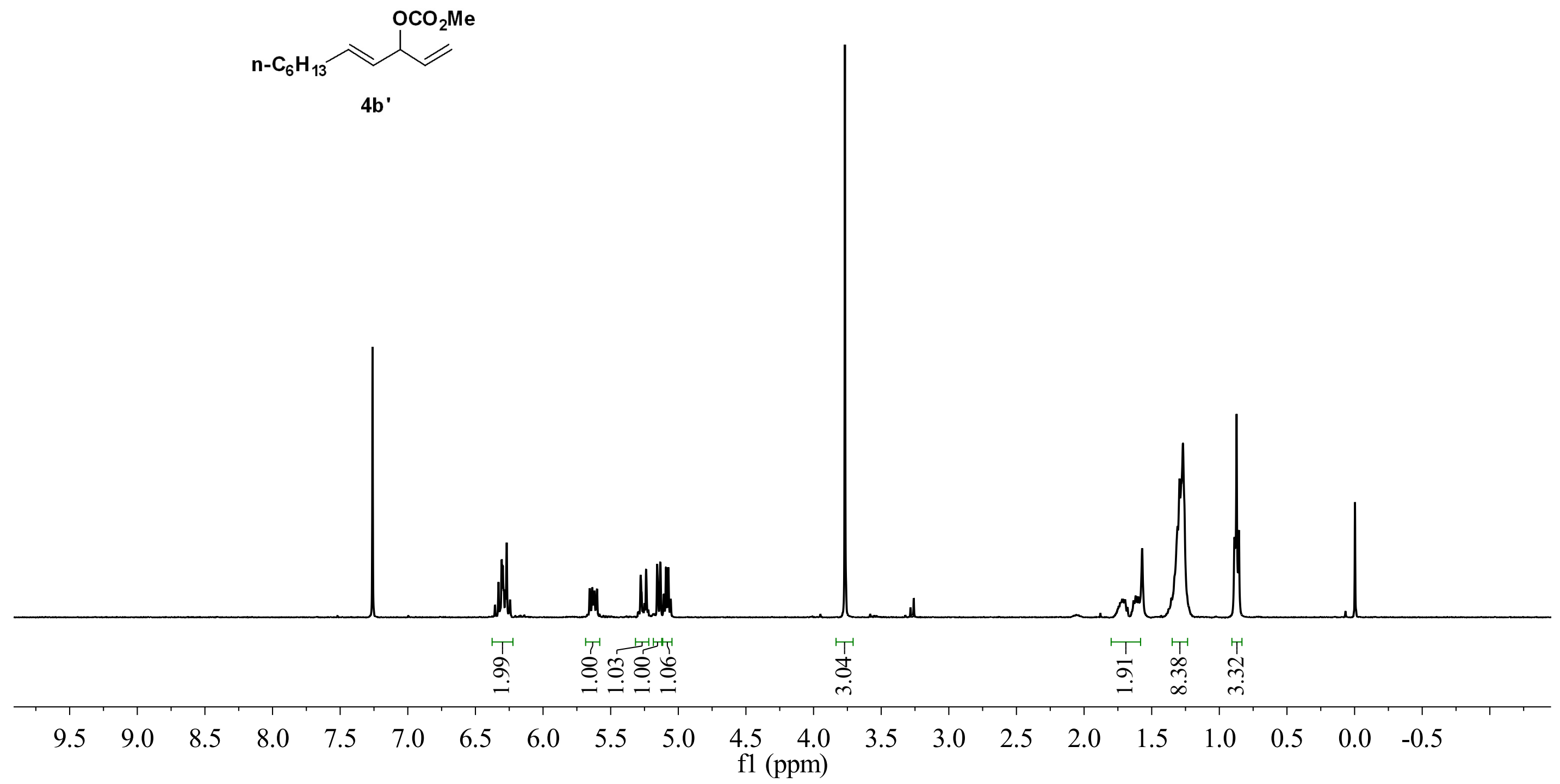

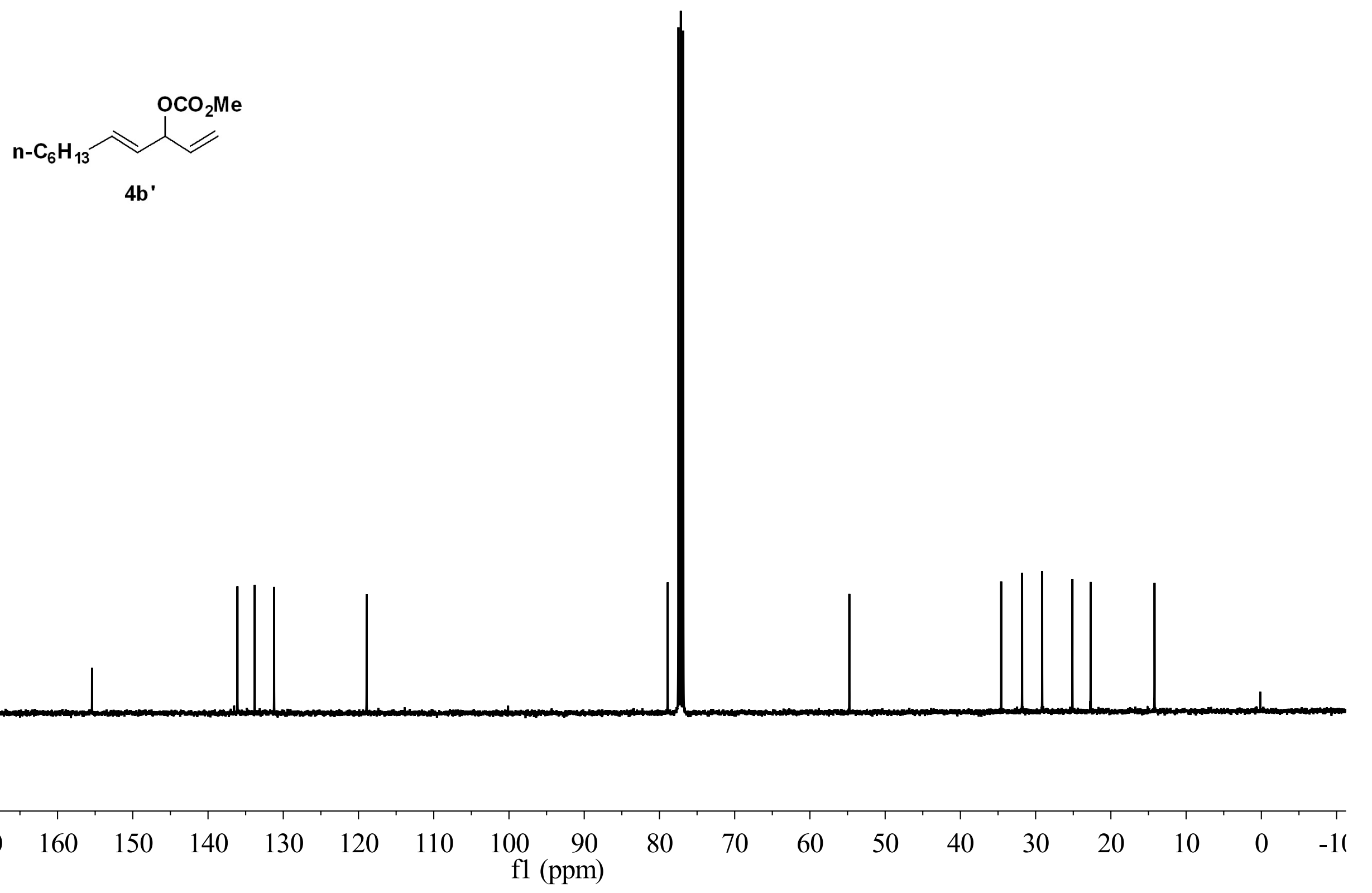


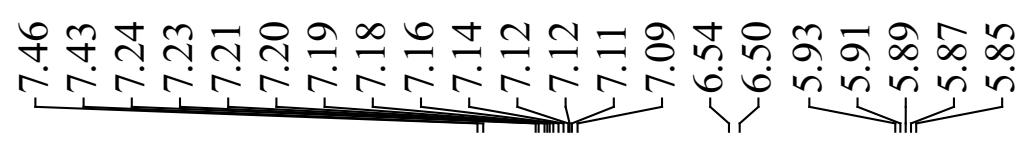

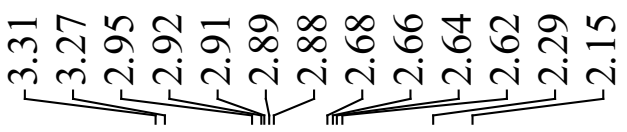
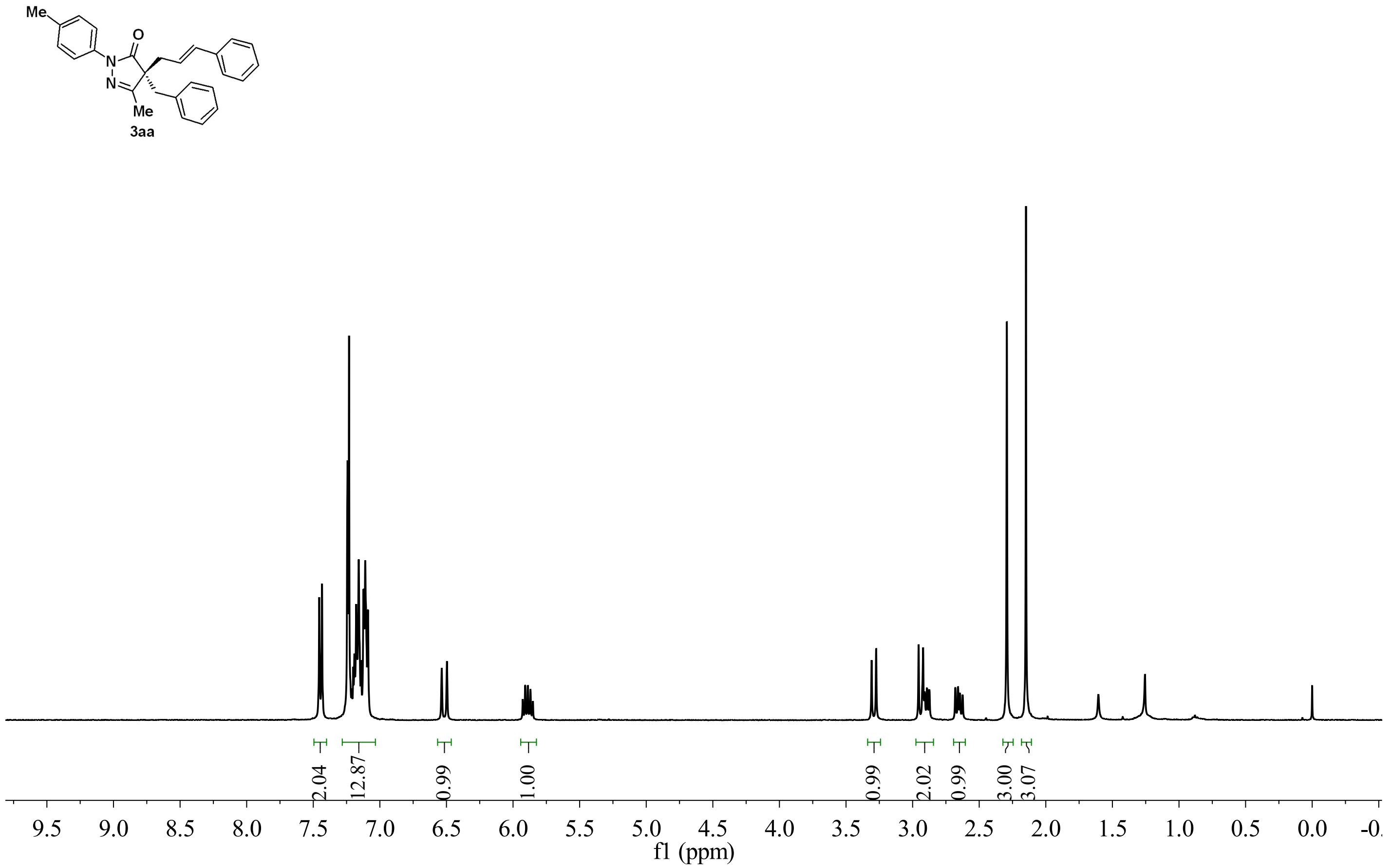

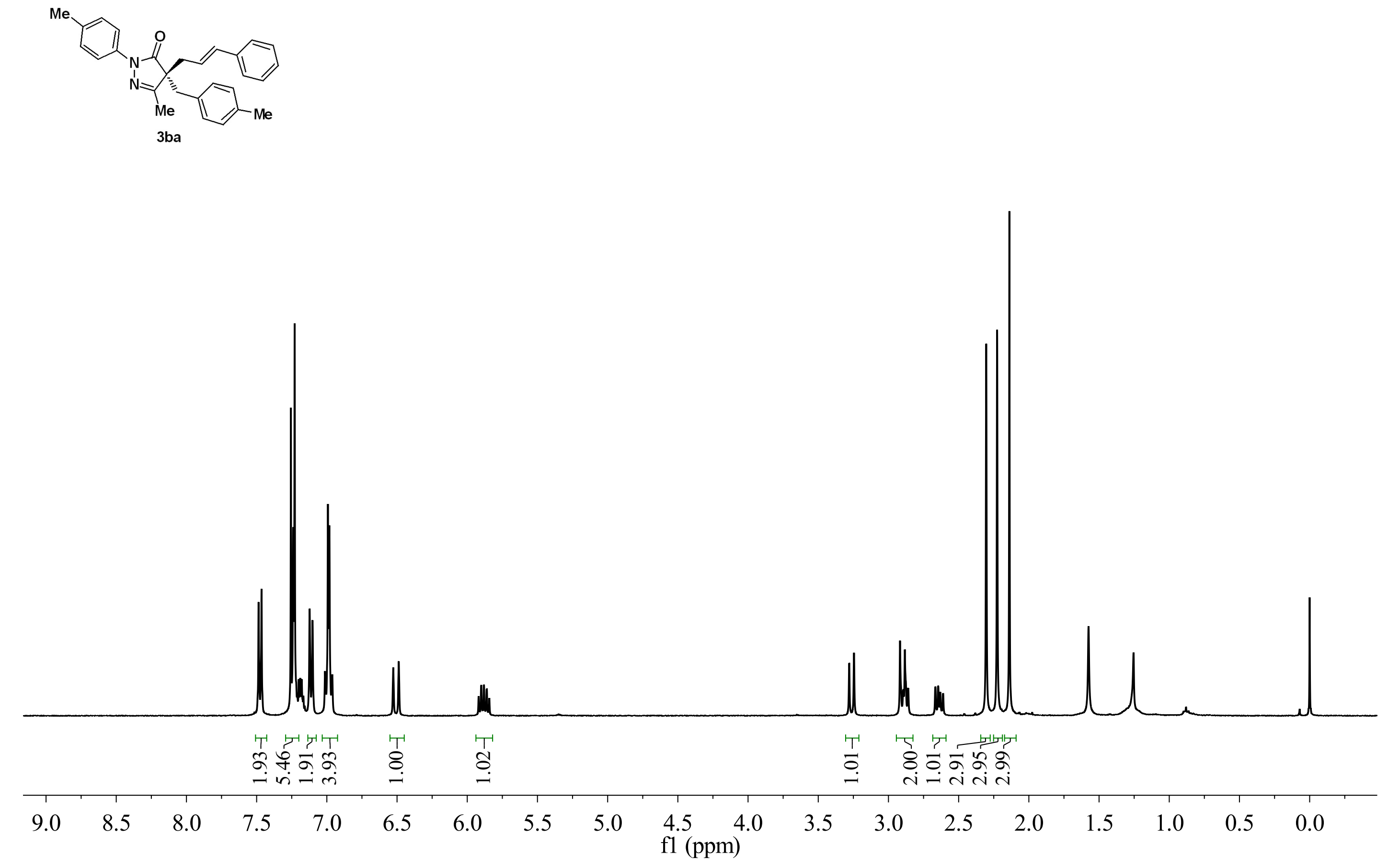

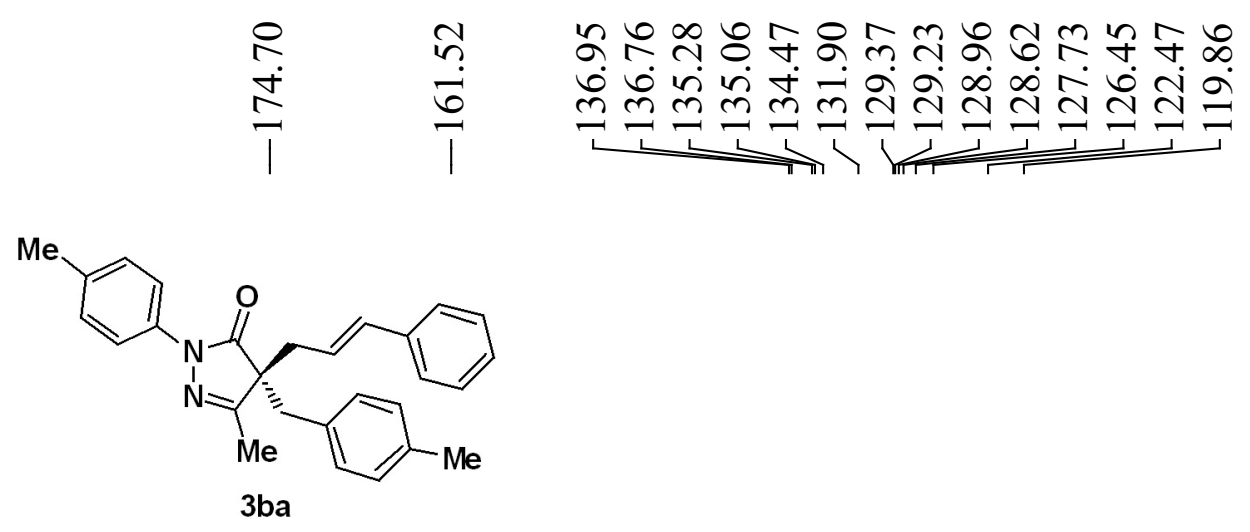

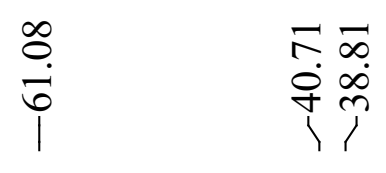

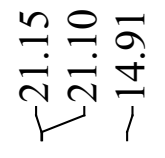
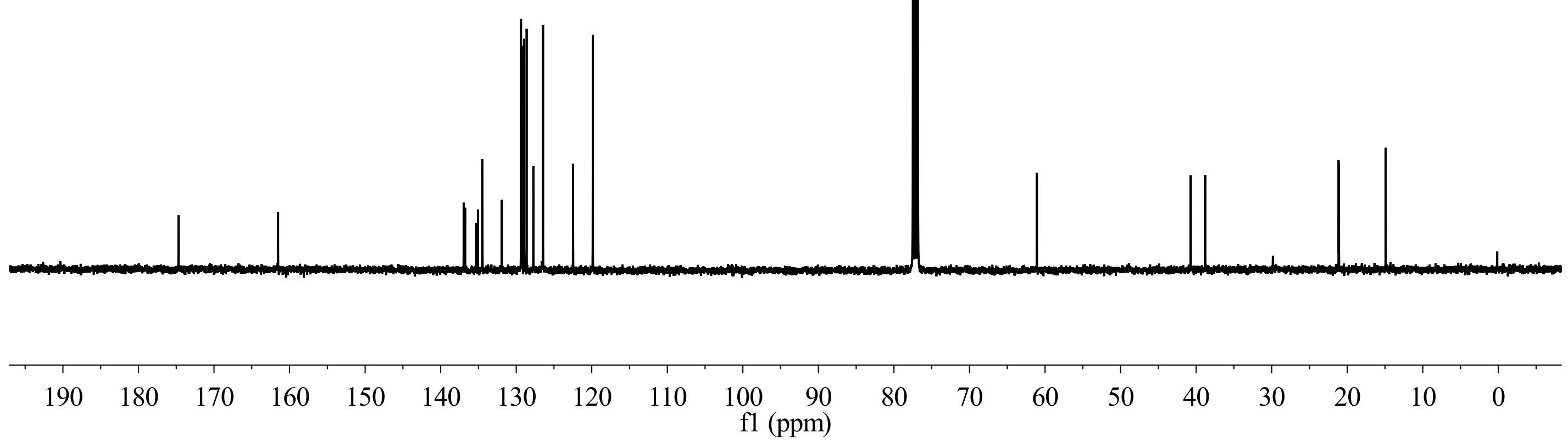


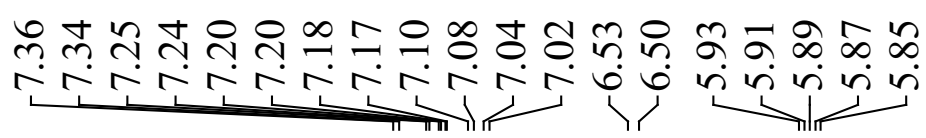

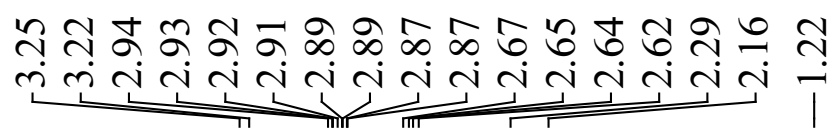
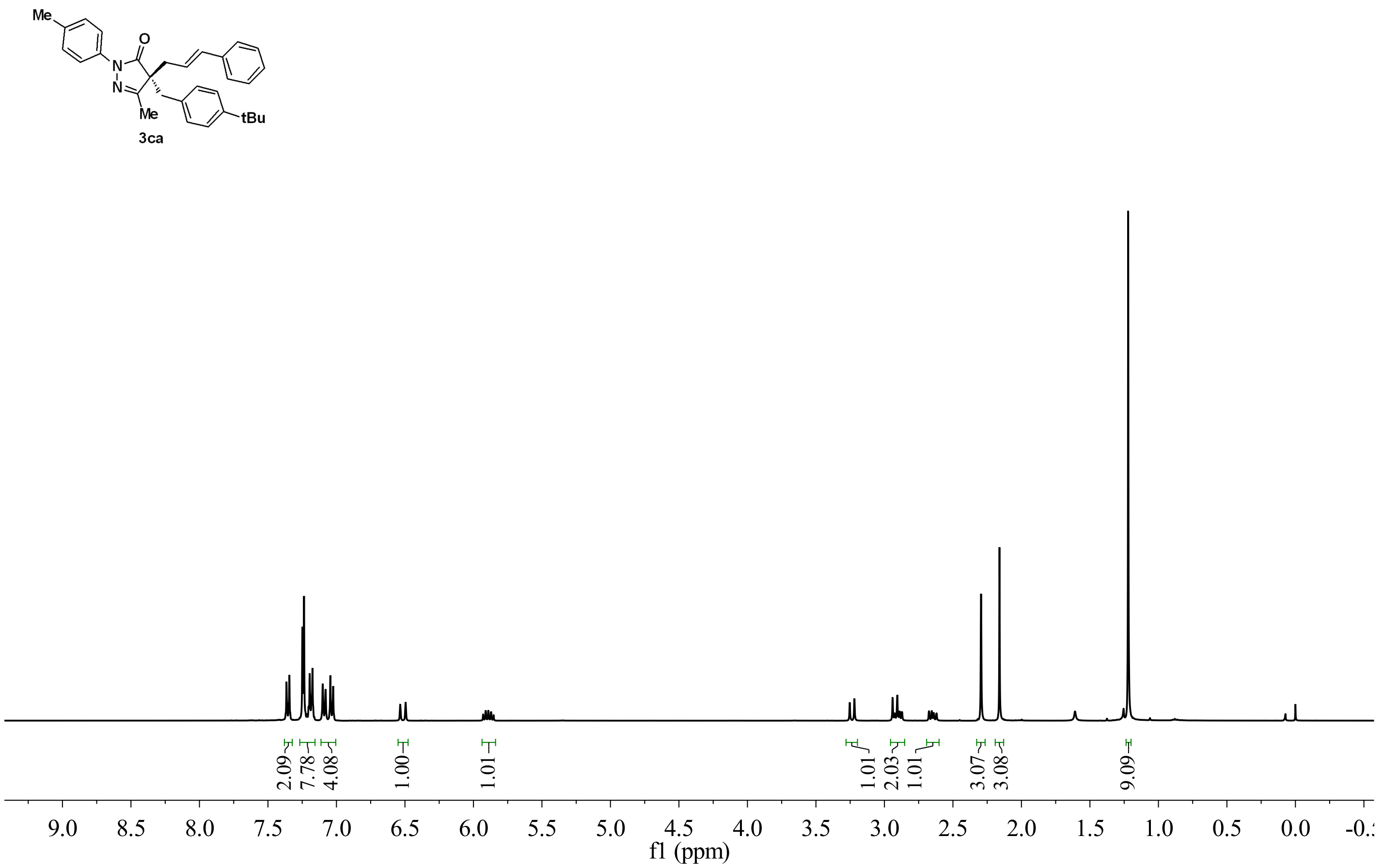


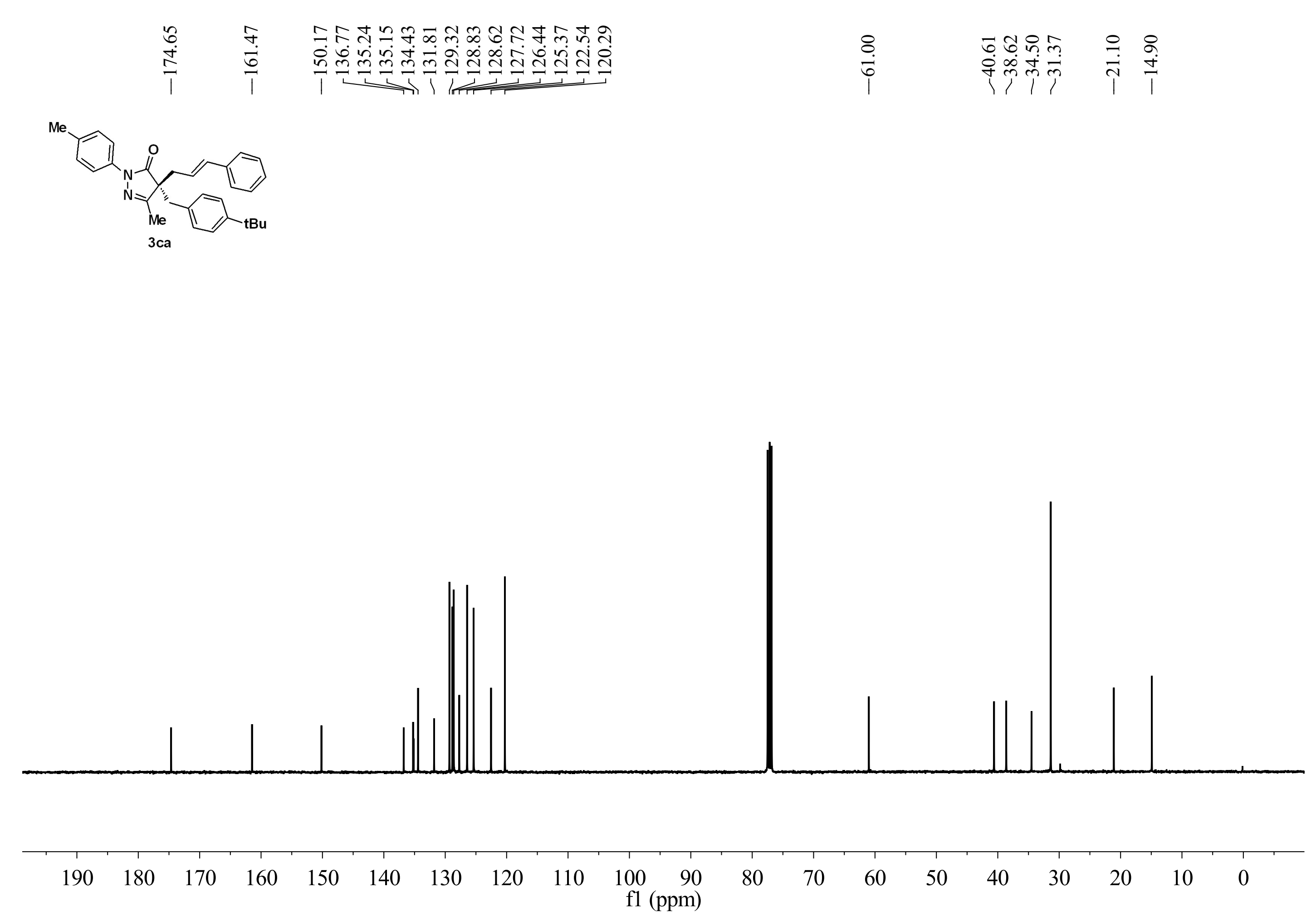




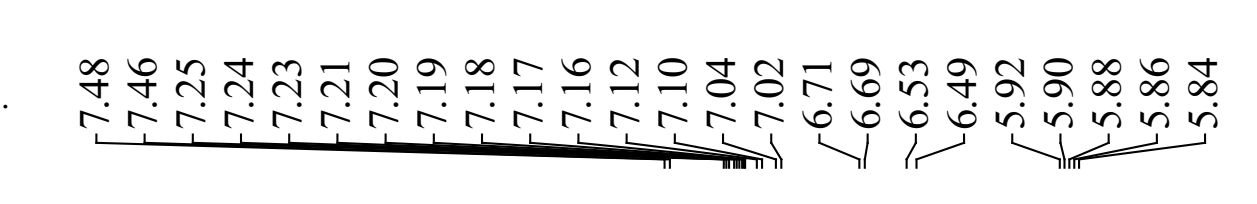

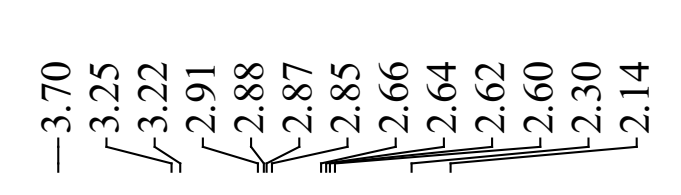
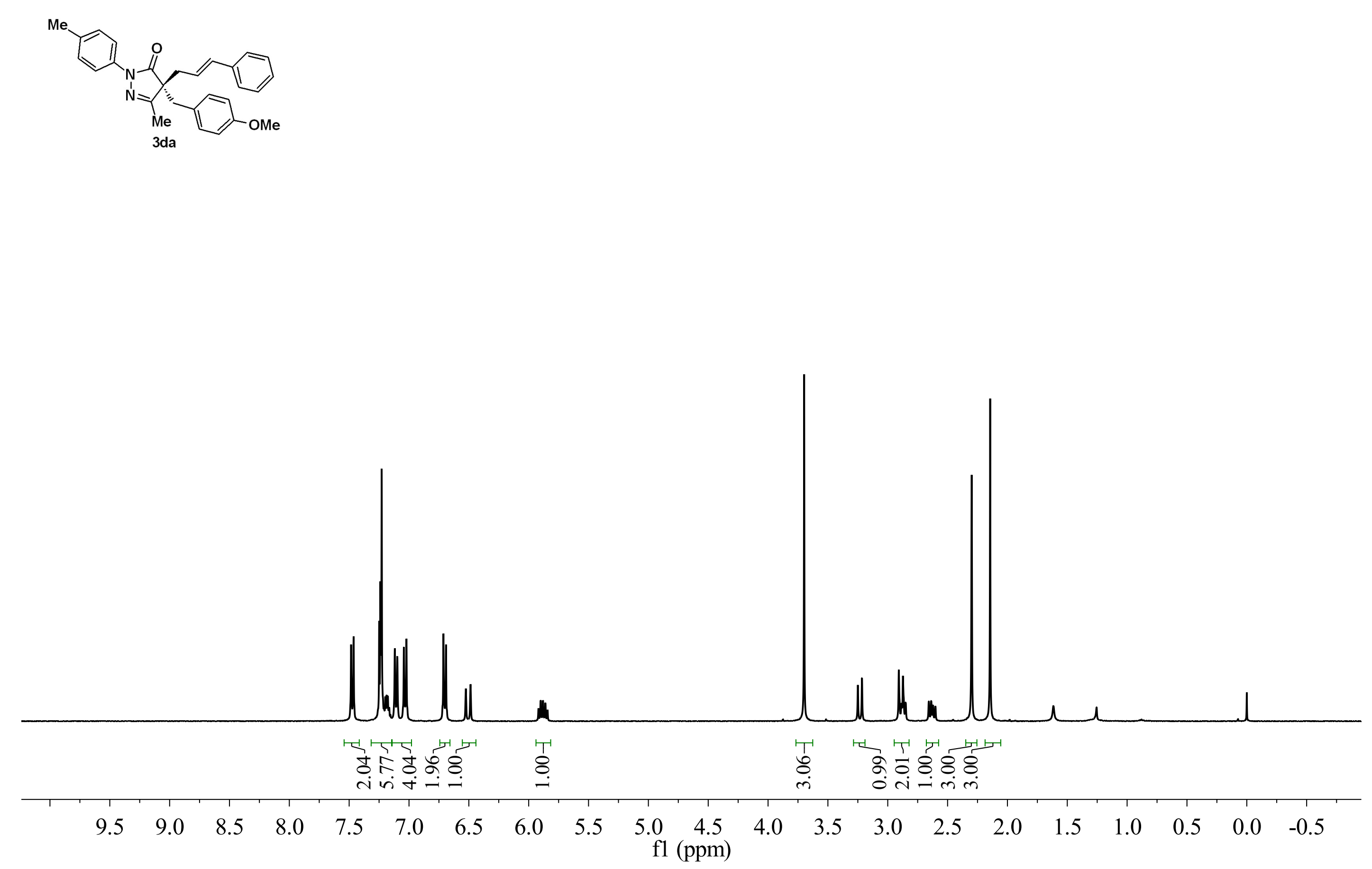


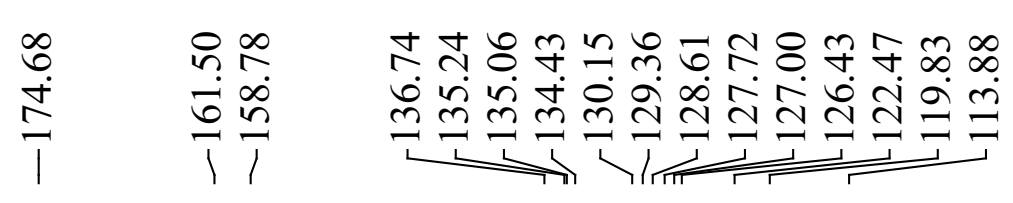

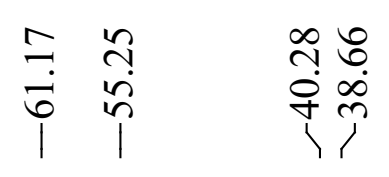

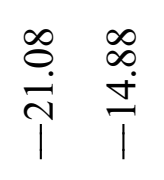
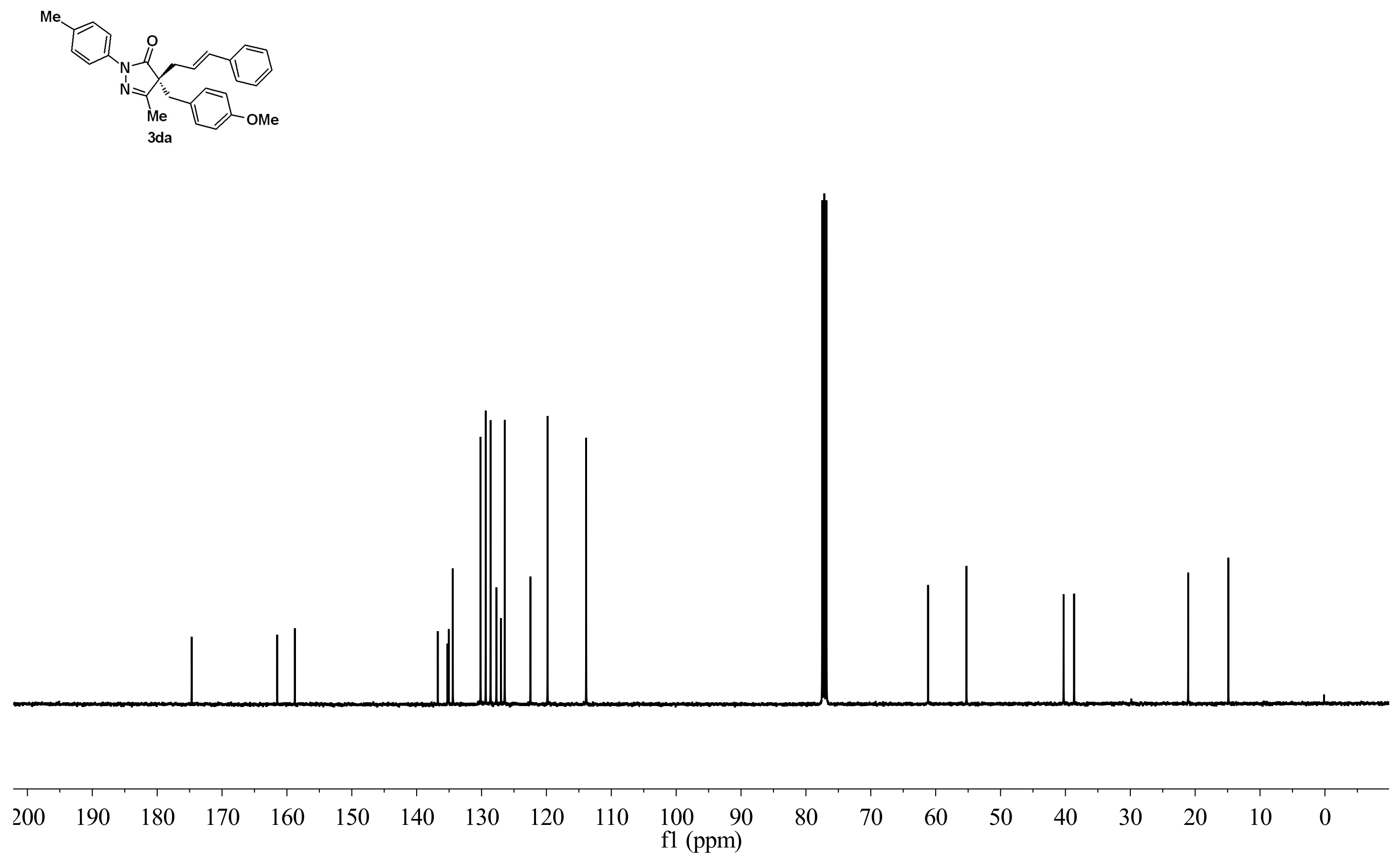

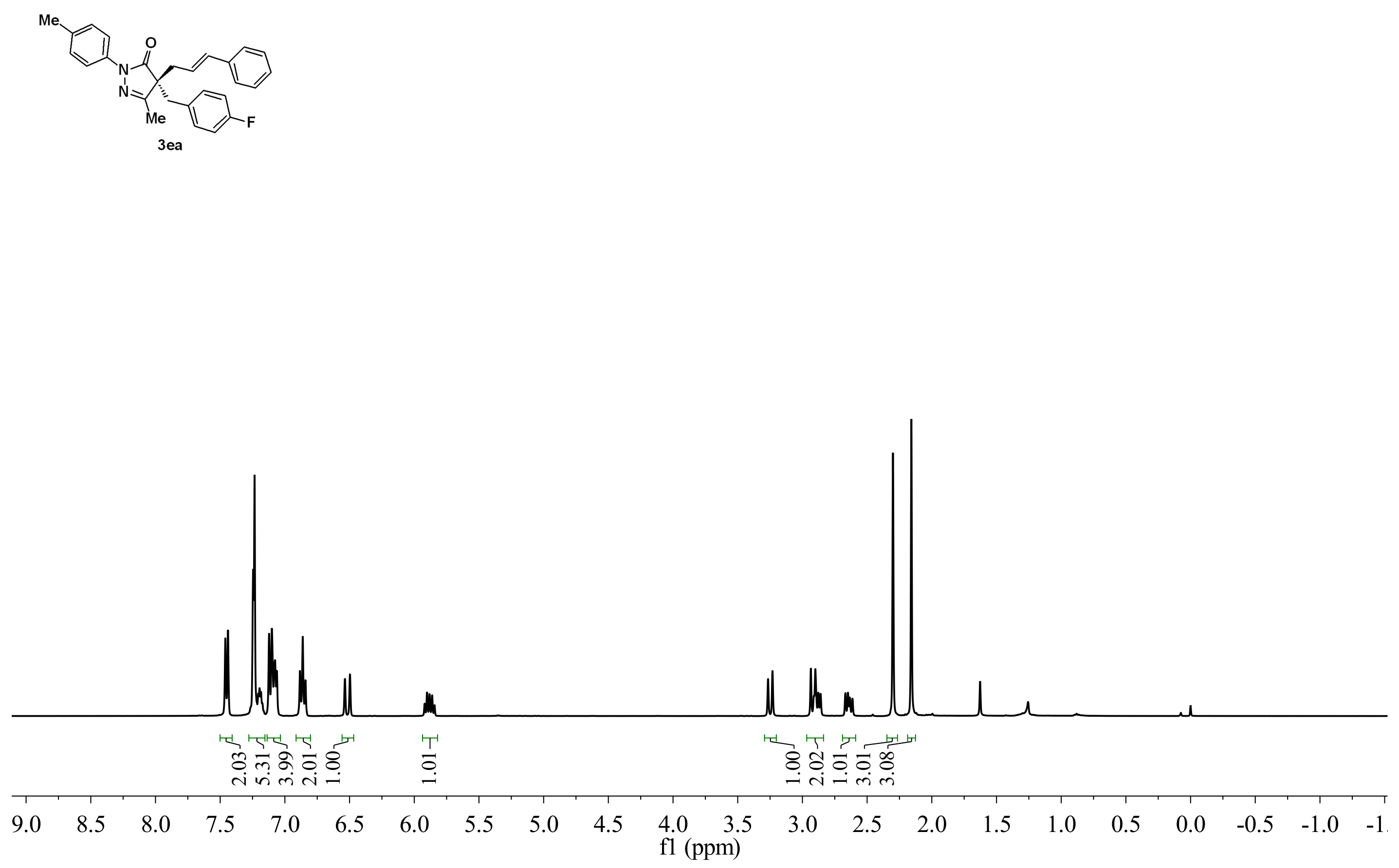


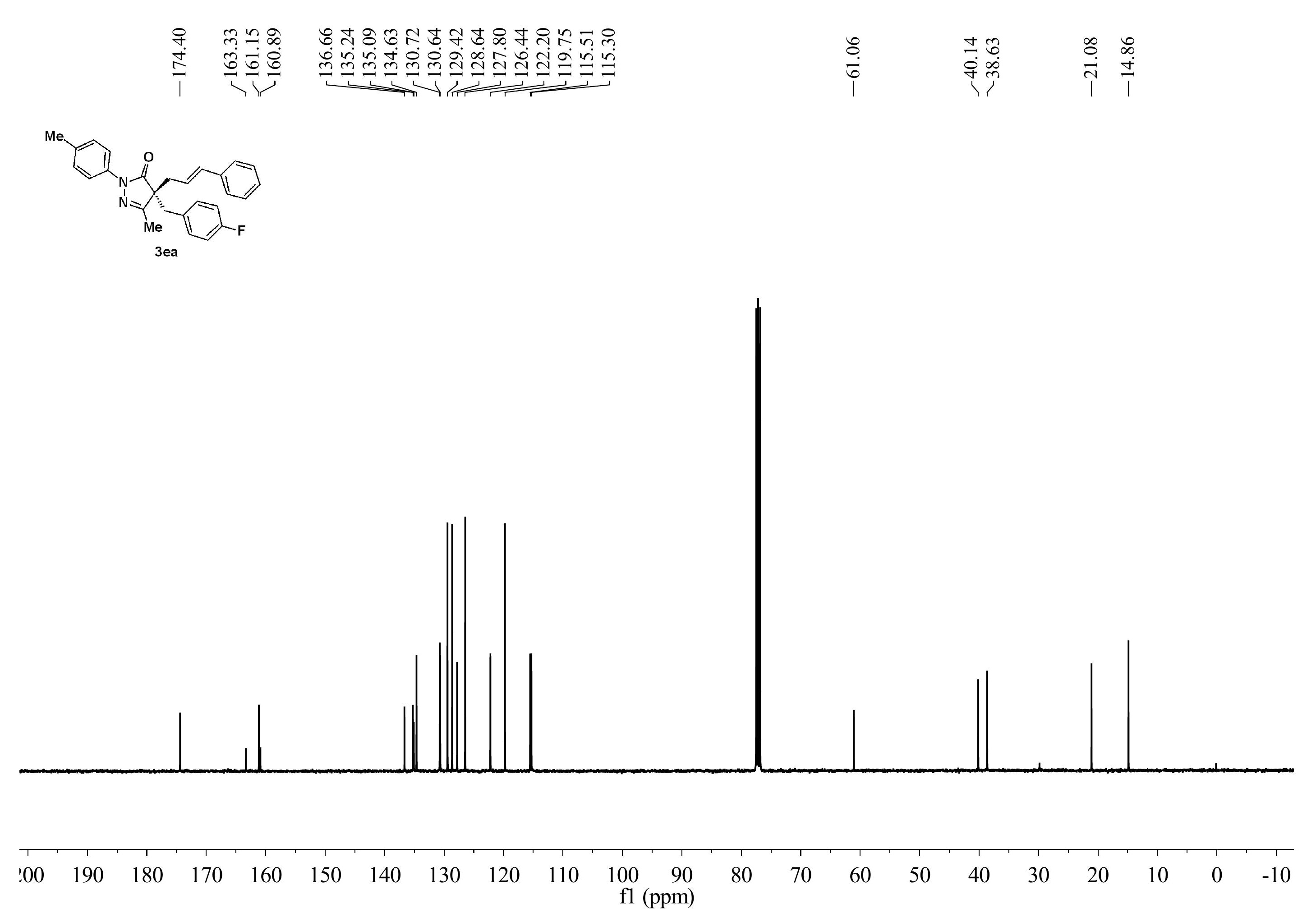



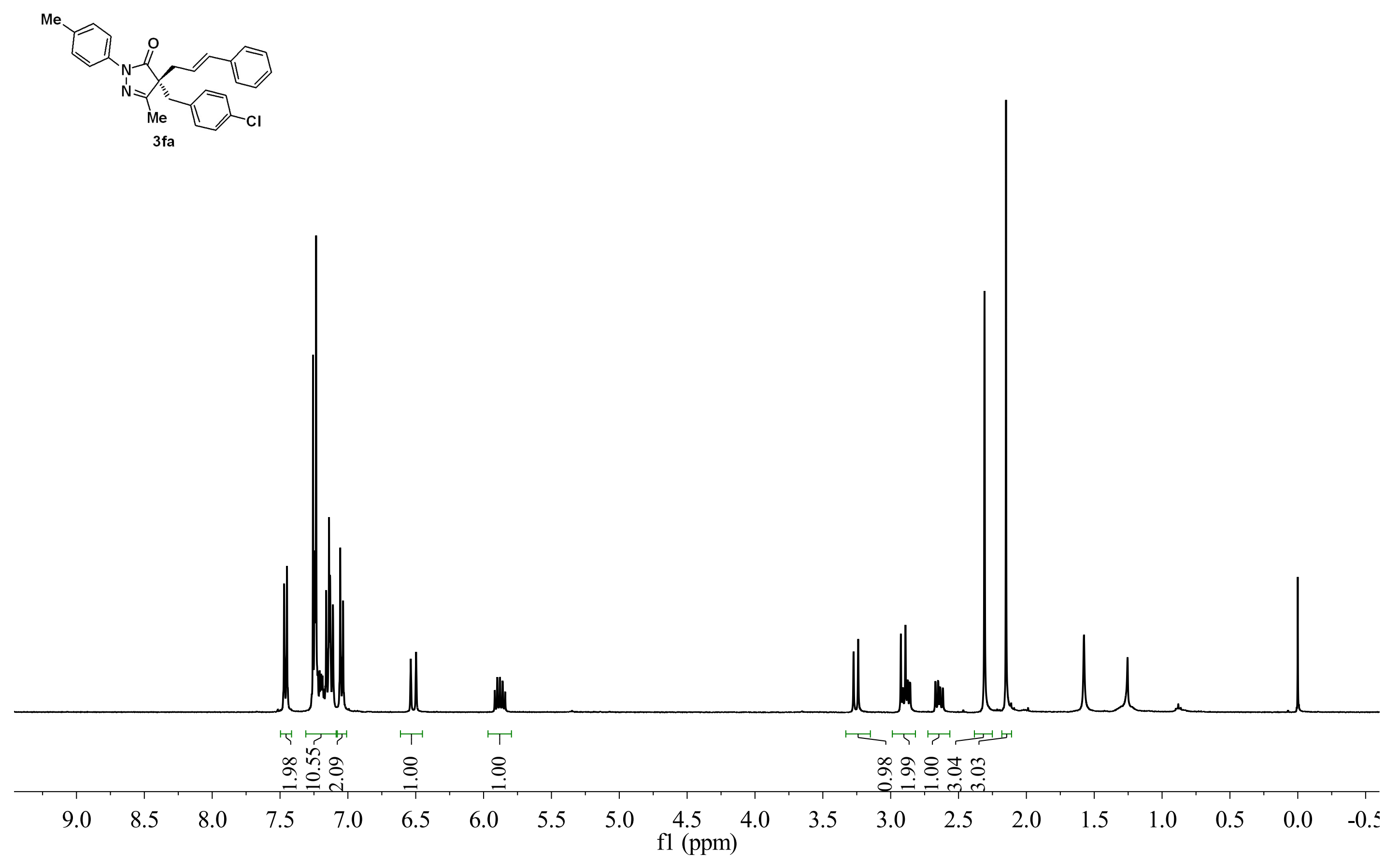
Me
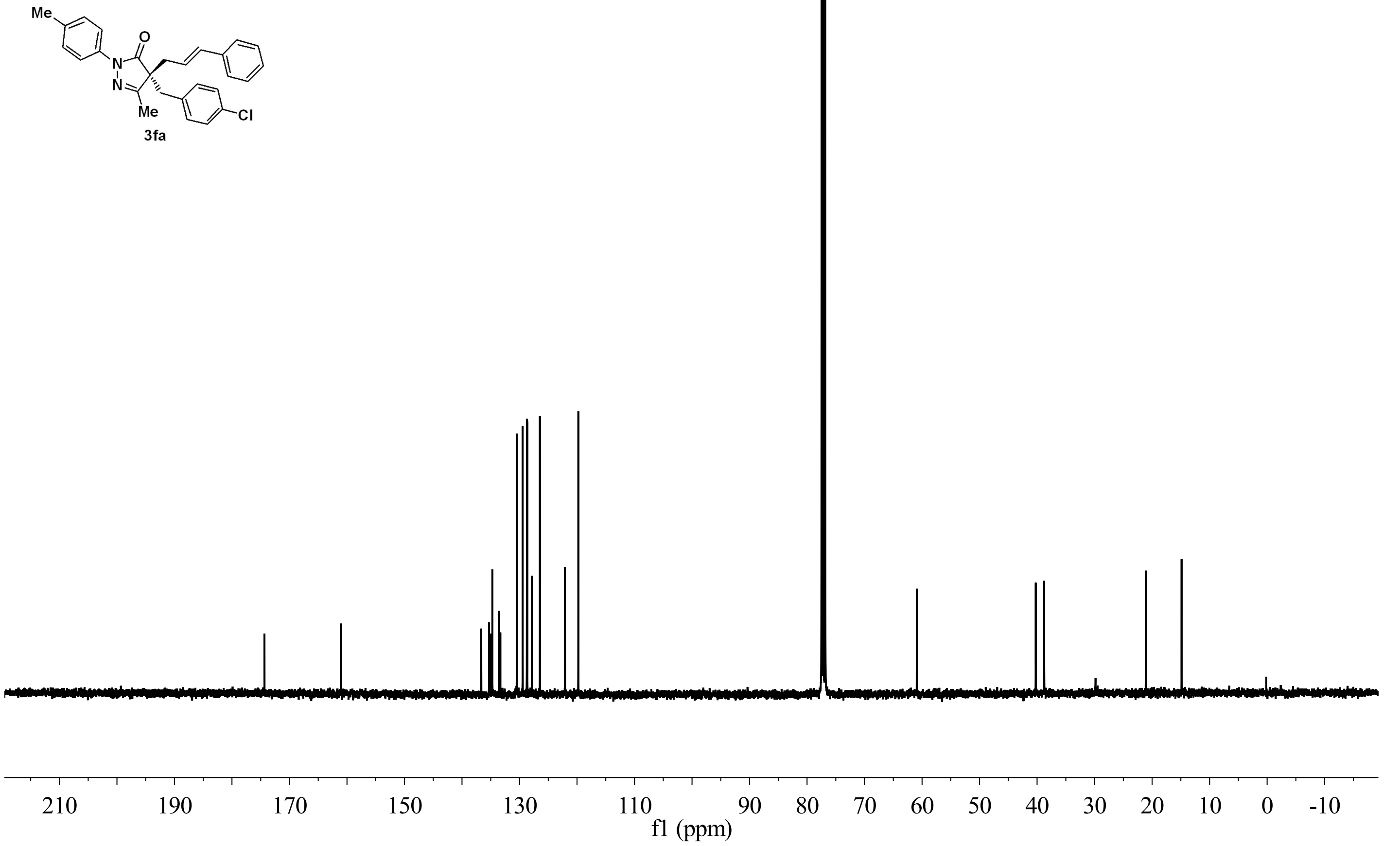


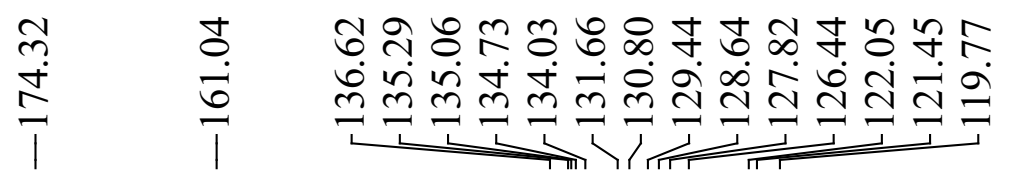

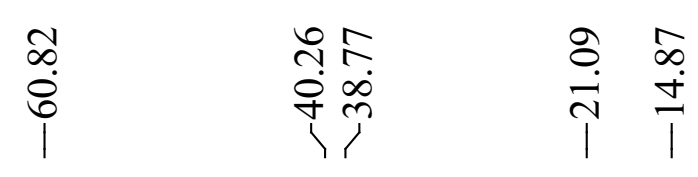
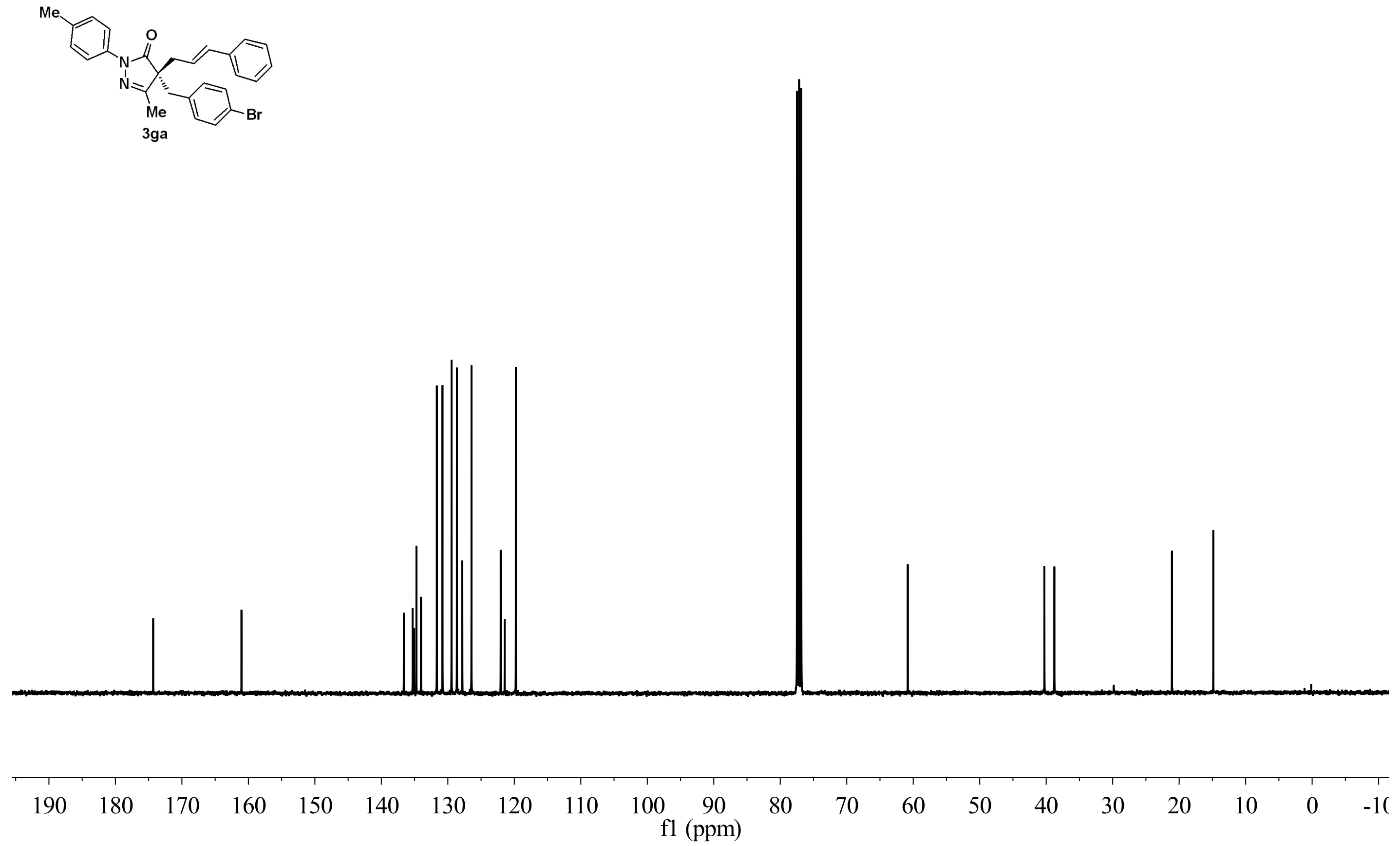

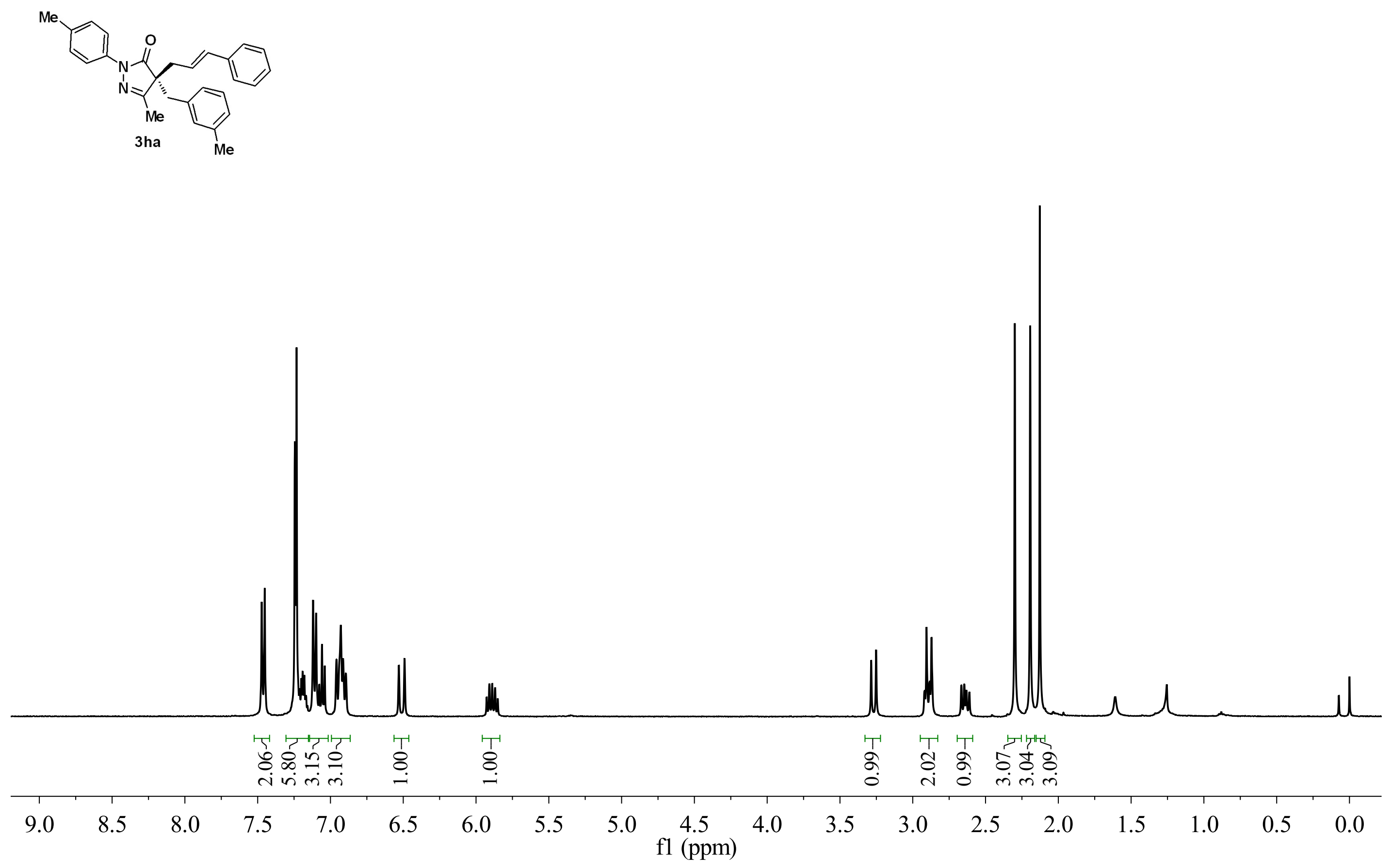
$\mathrm{Me}$
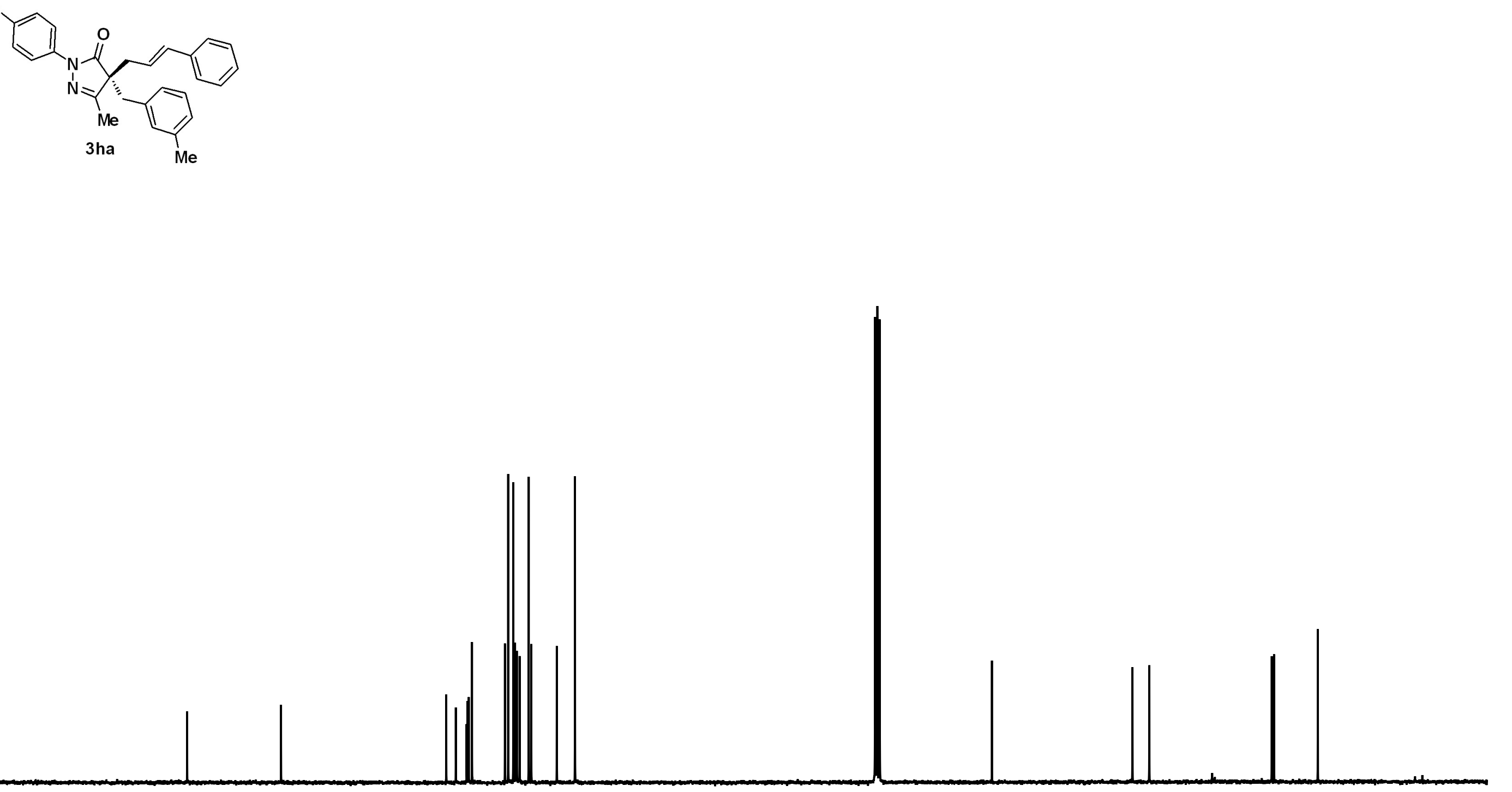

$\begin{array}{lllllllllllllllllllll}200 & 190 & 180 & 170 & 160 & 150 & 140 & 130 & 120 & 110 & 100 & 90 & 80 & 70 & 60 & 50 & 40 & 30 & 20 & 10 & 0\end{array}$




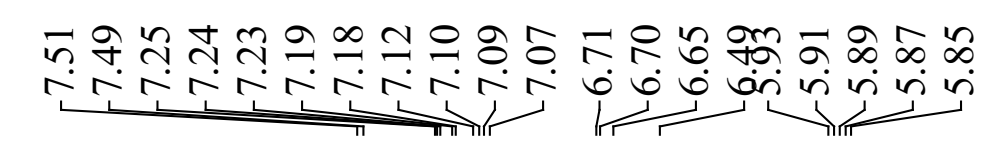

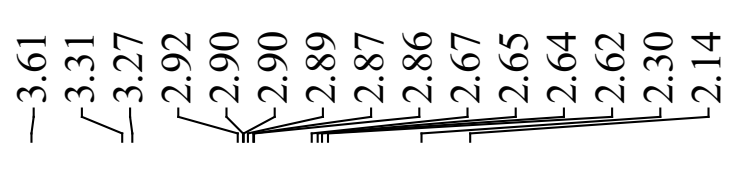
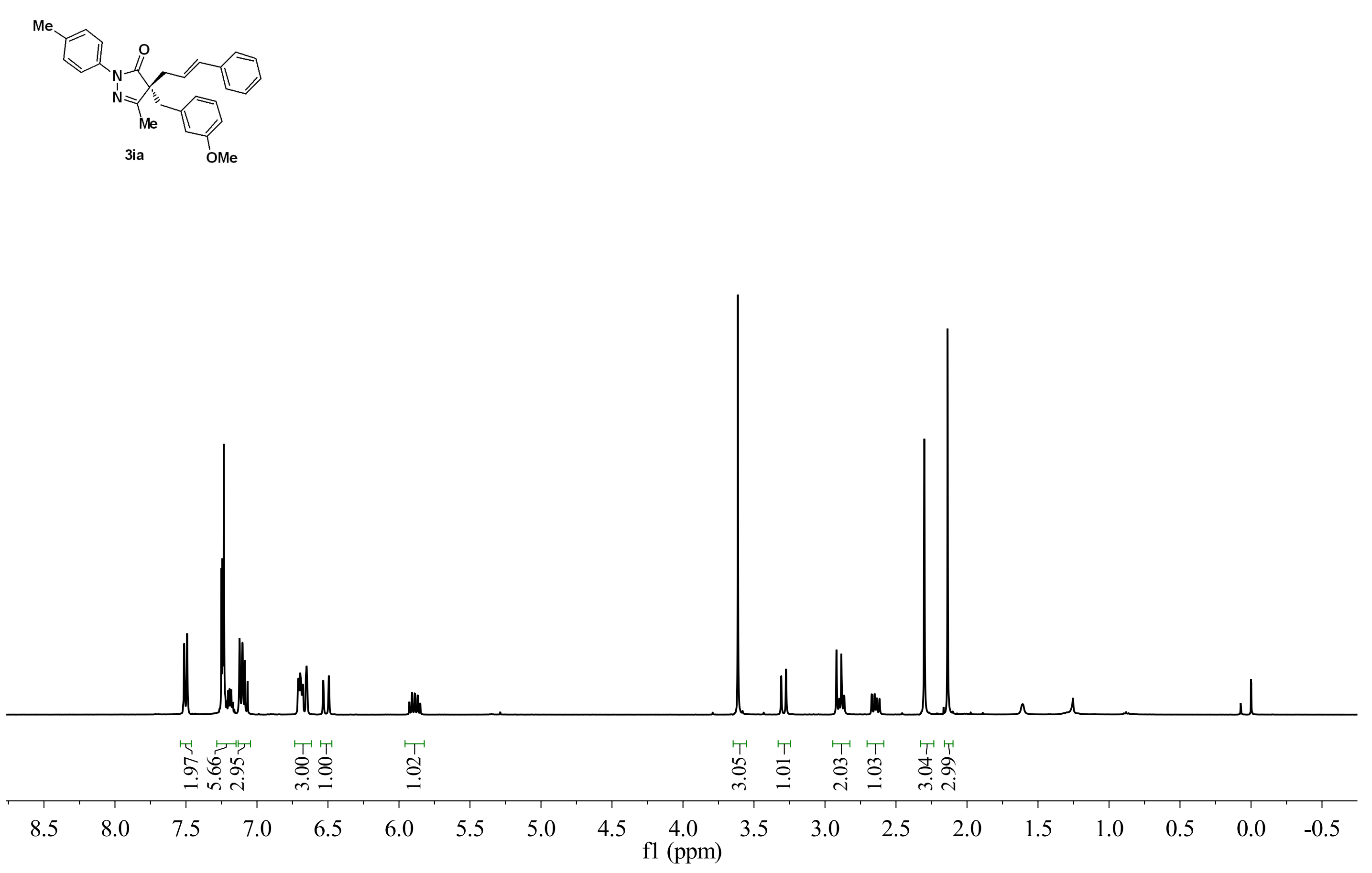


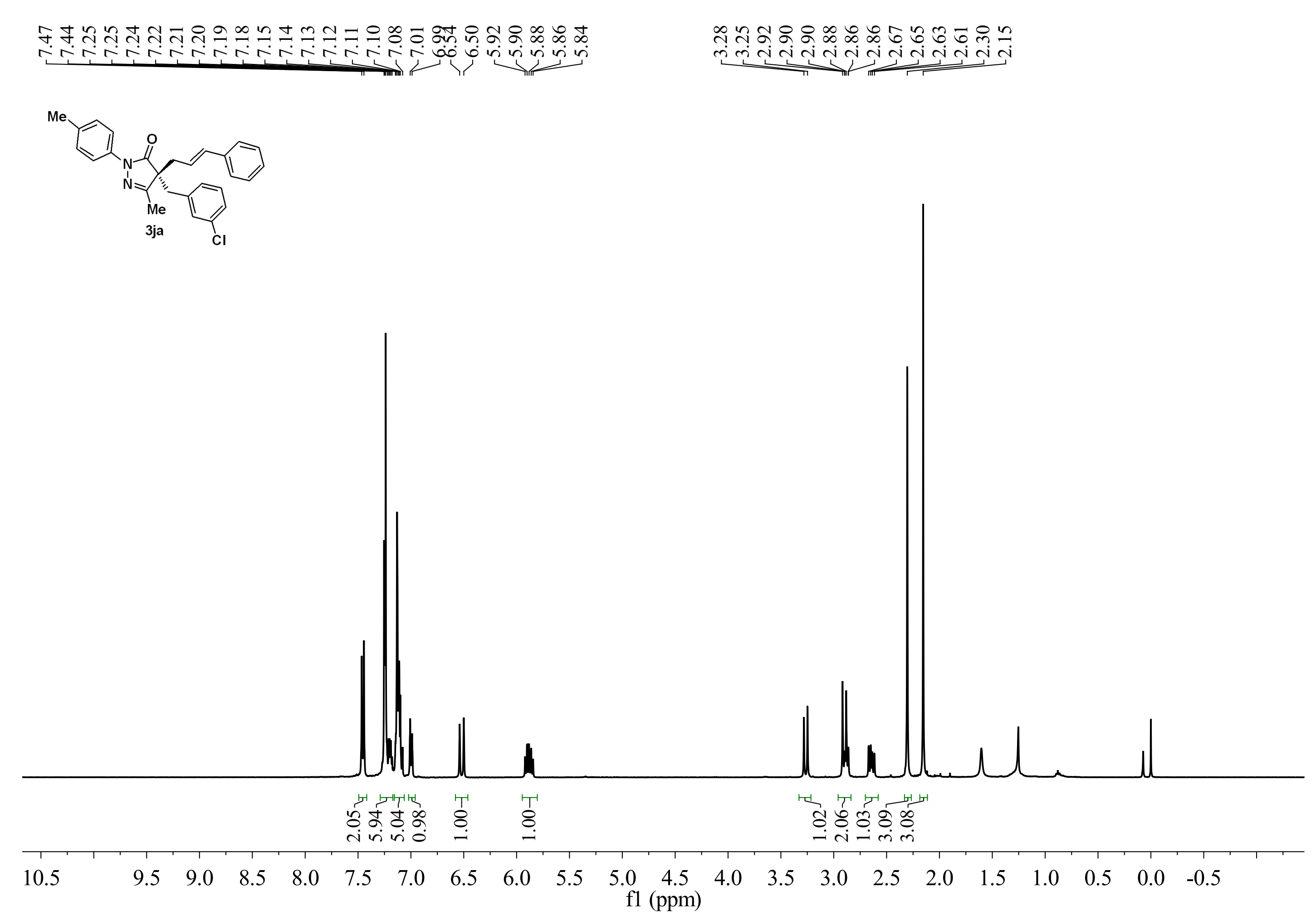




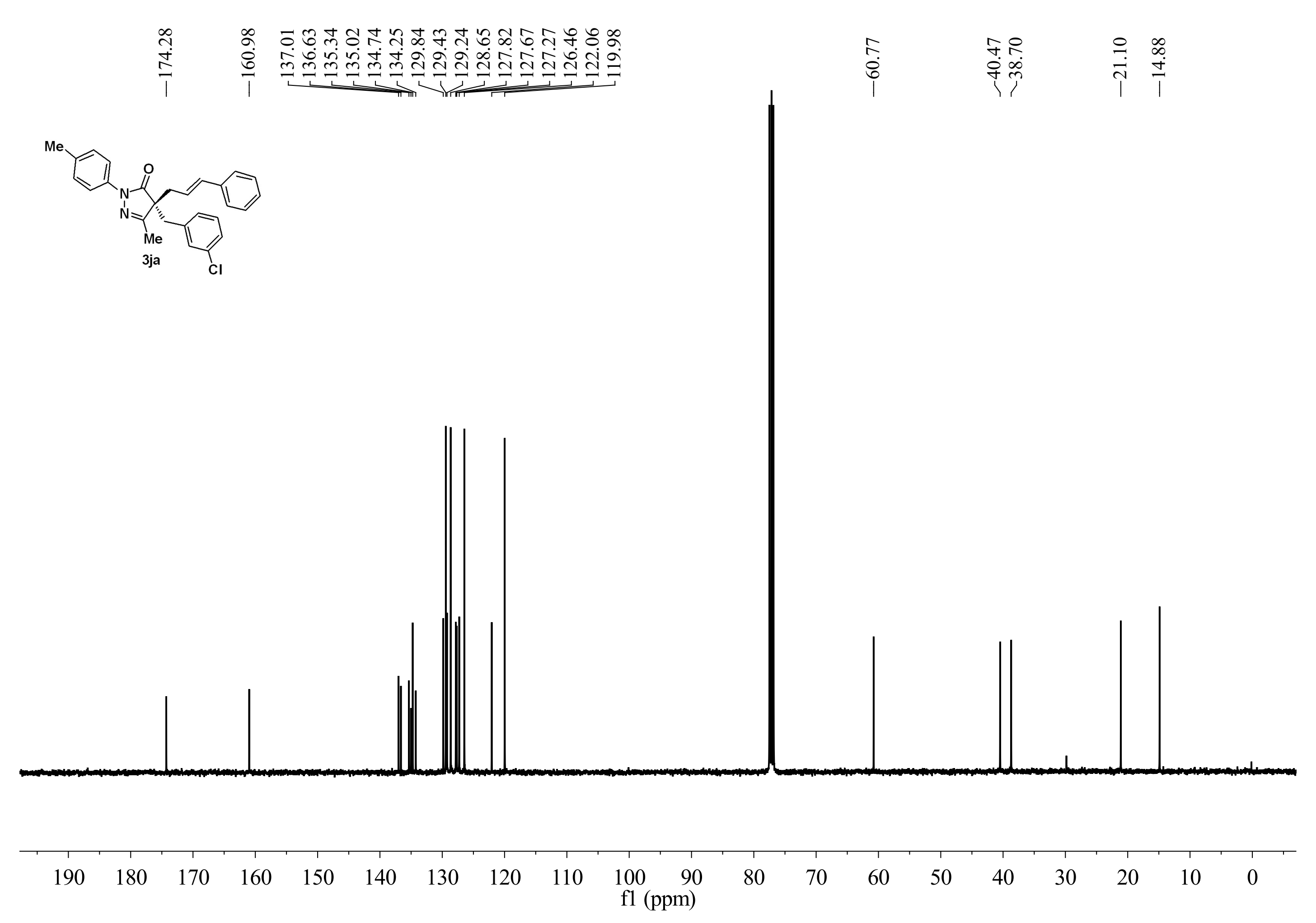



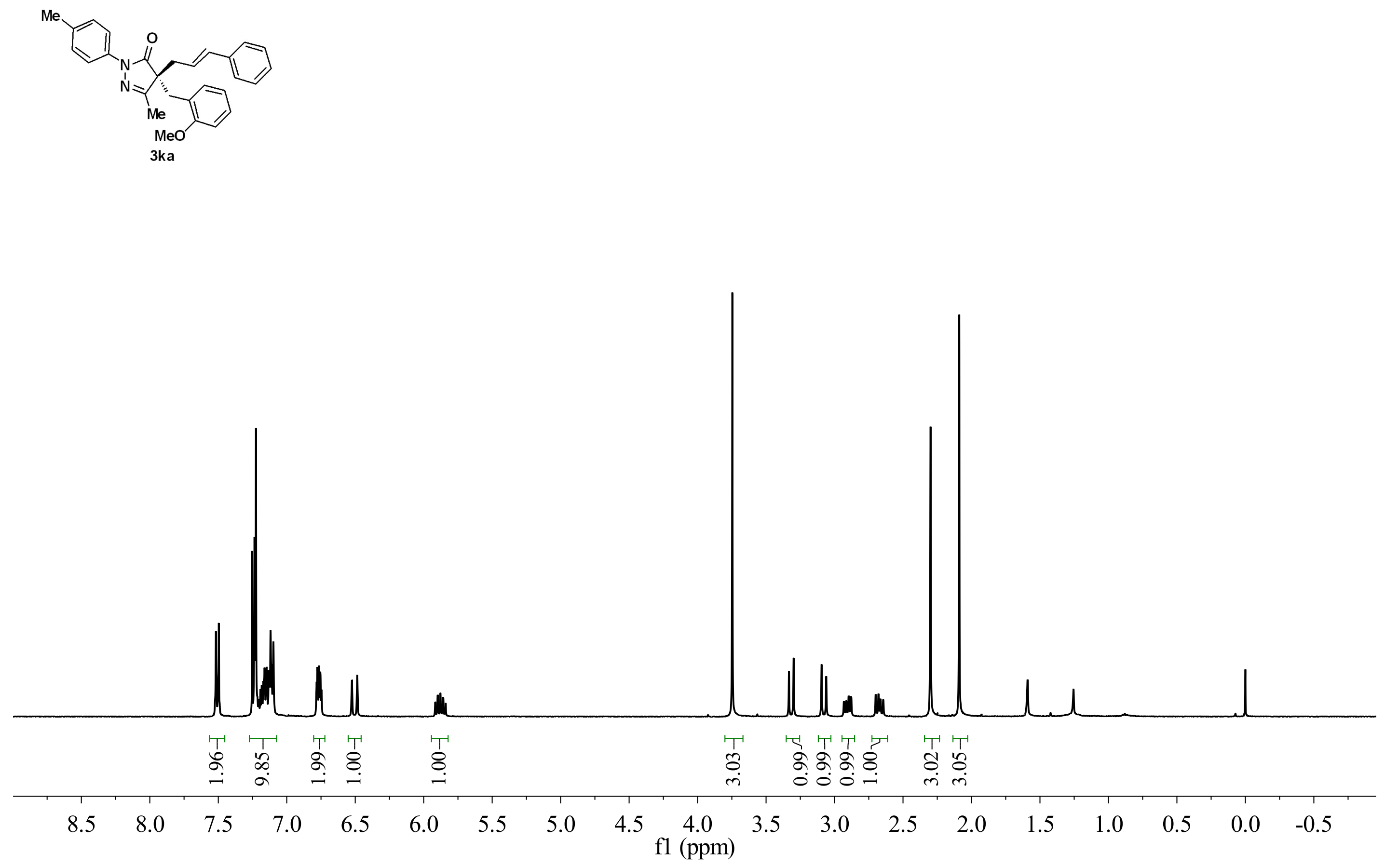
$\mathrm{Me}$
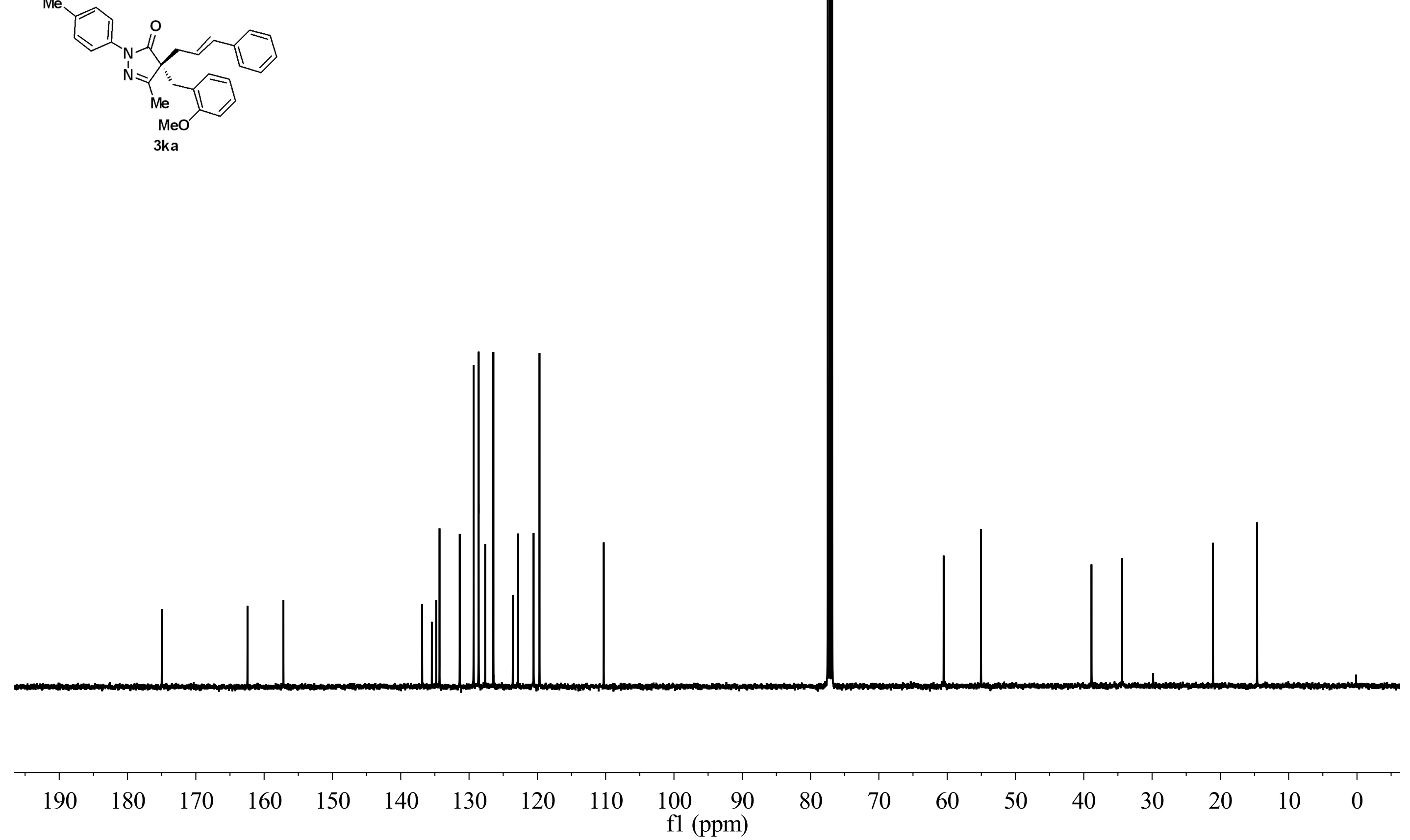
Me

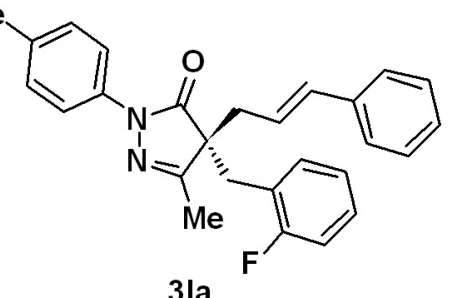

3la

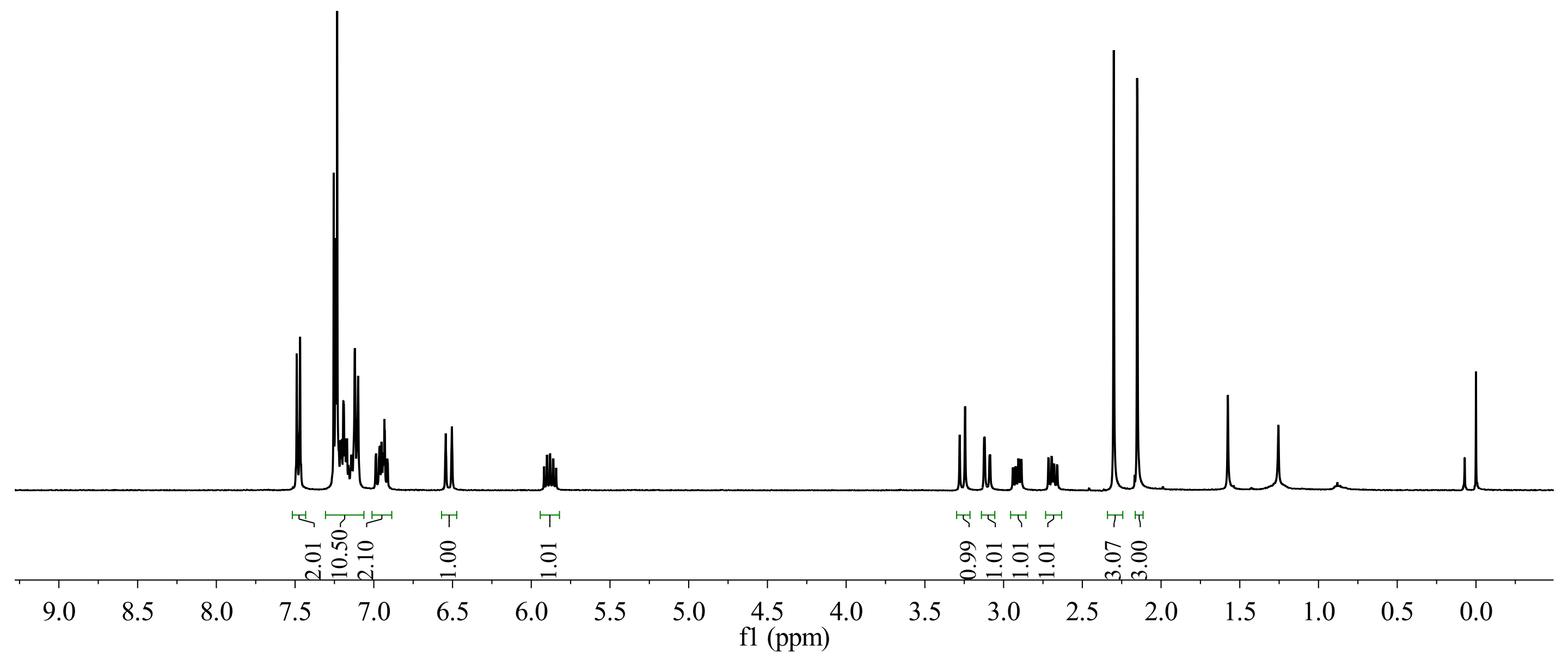




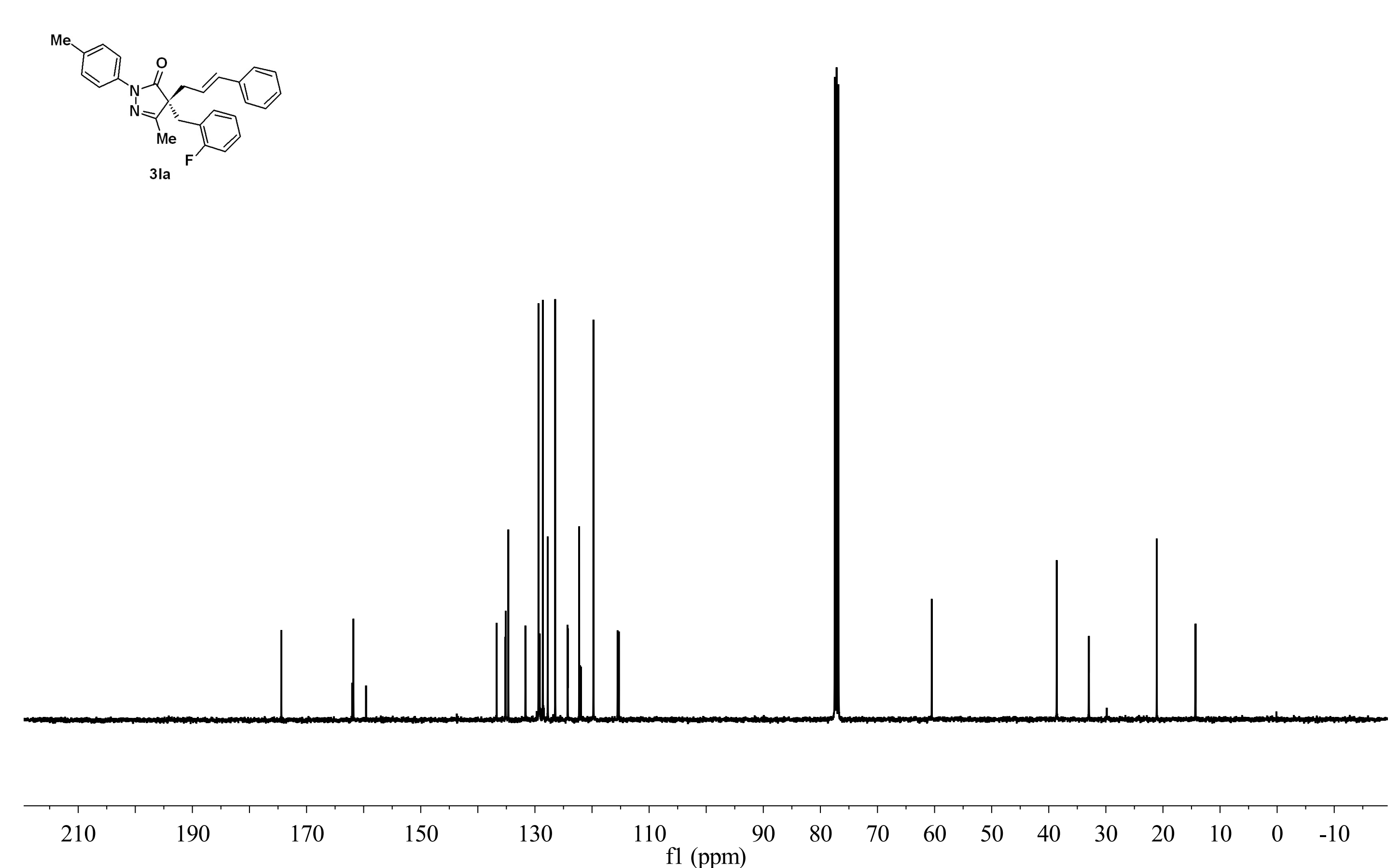




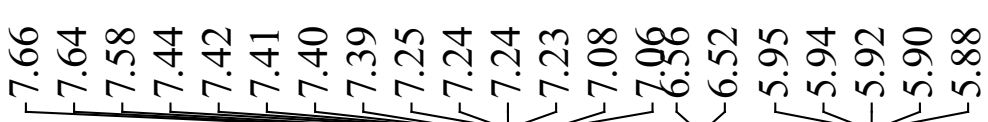

Me

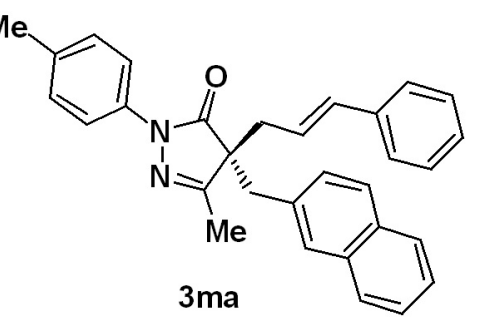

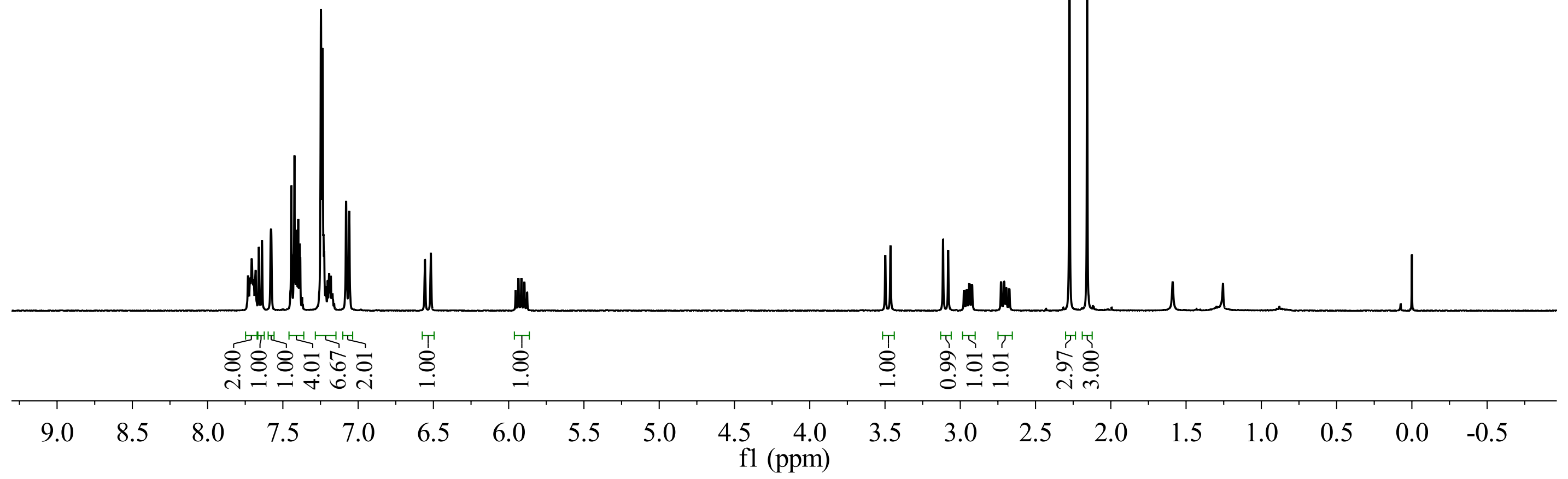




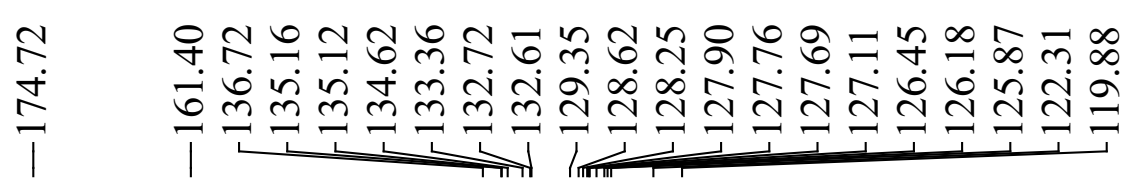

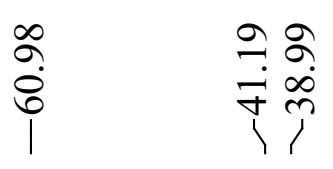

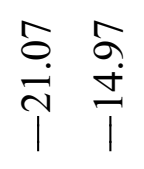
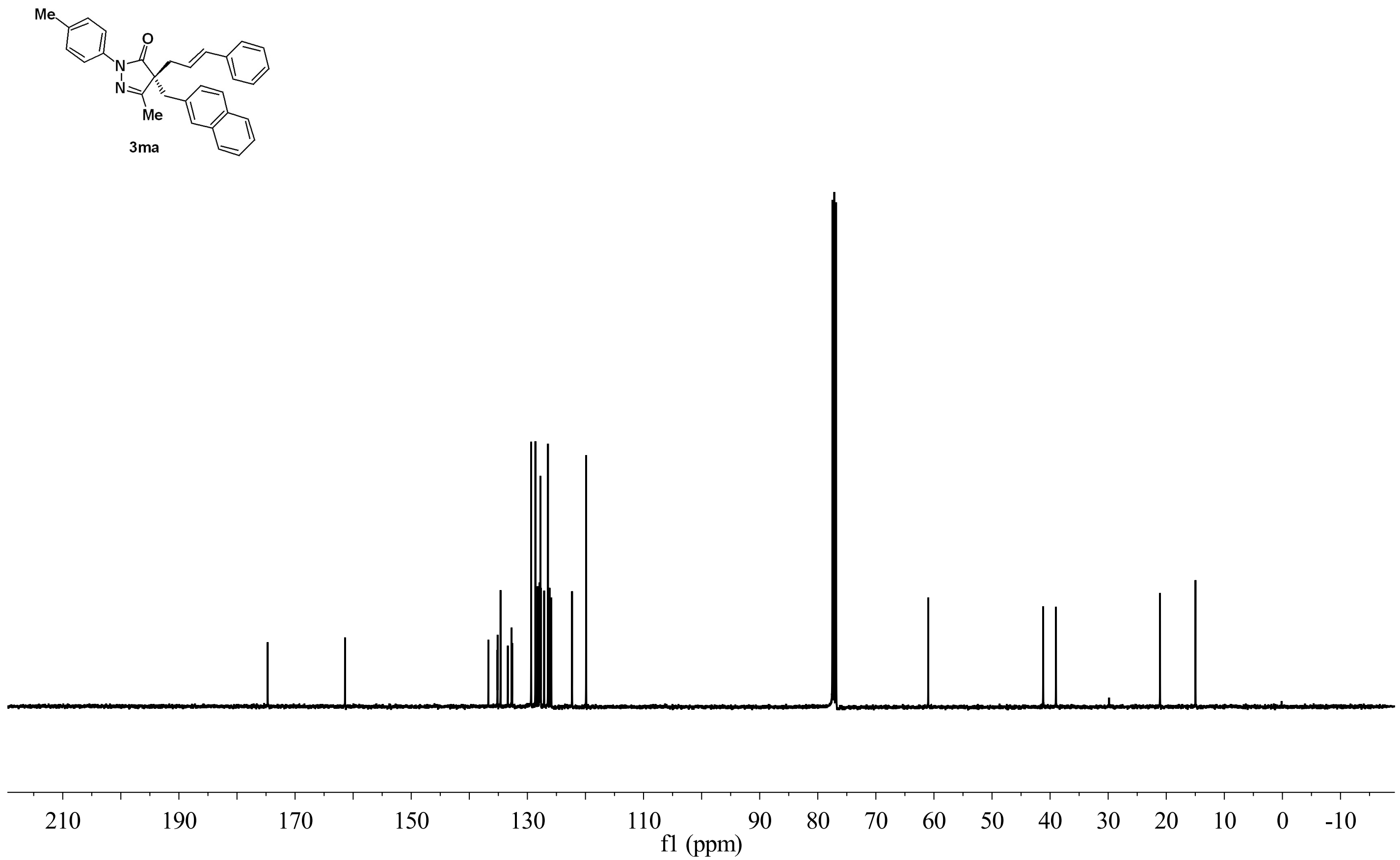
$\mathrm{Me}$

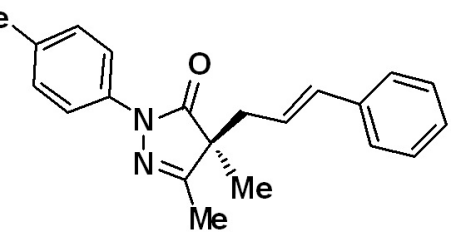

3na

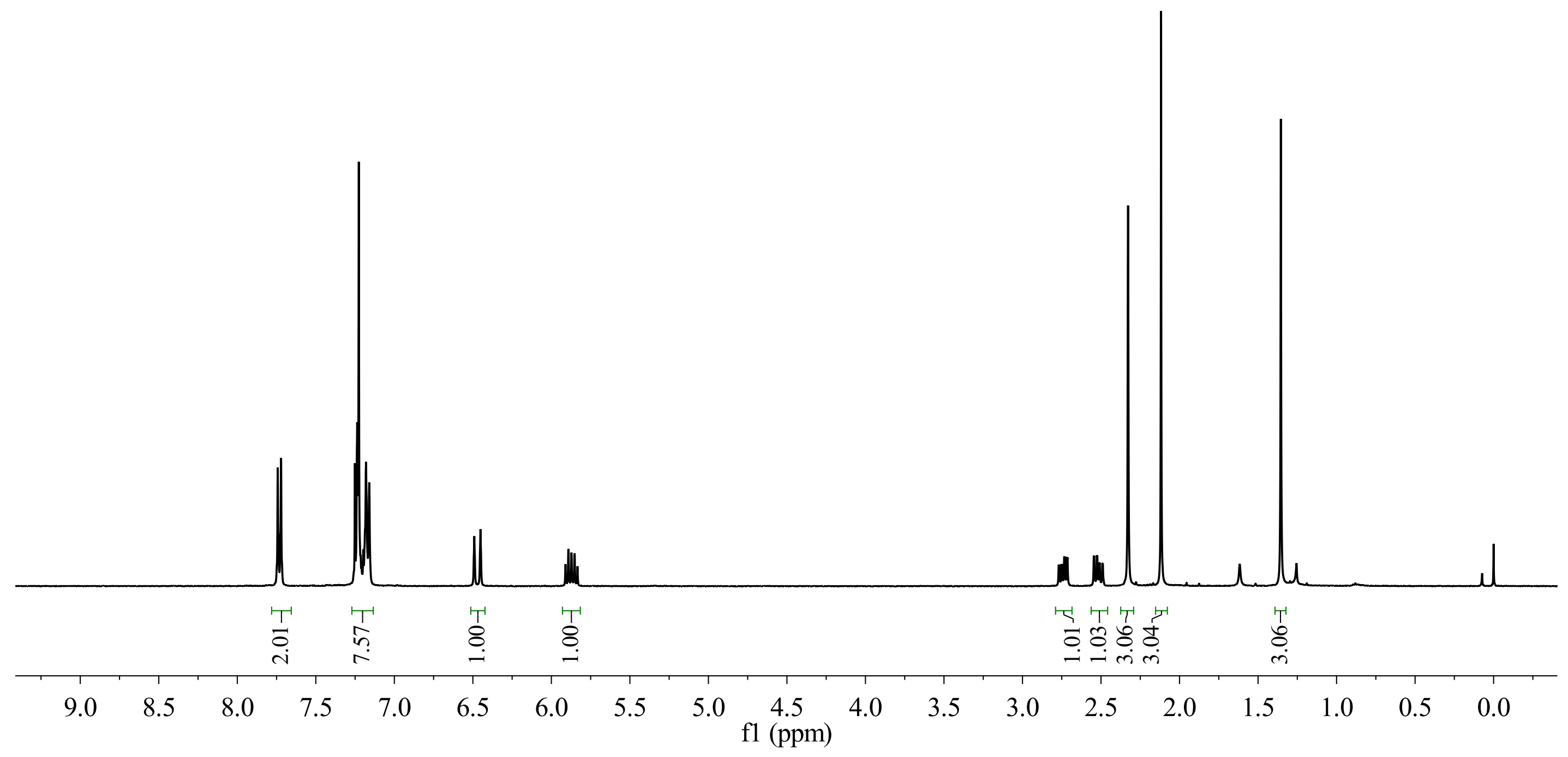



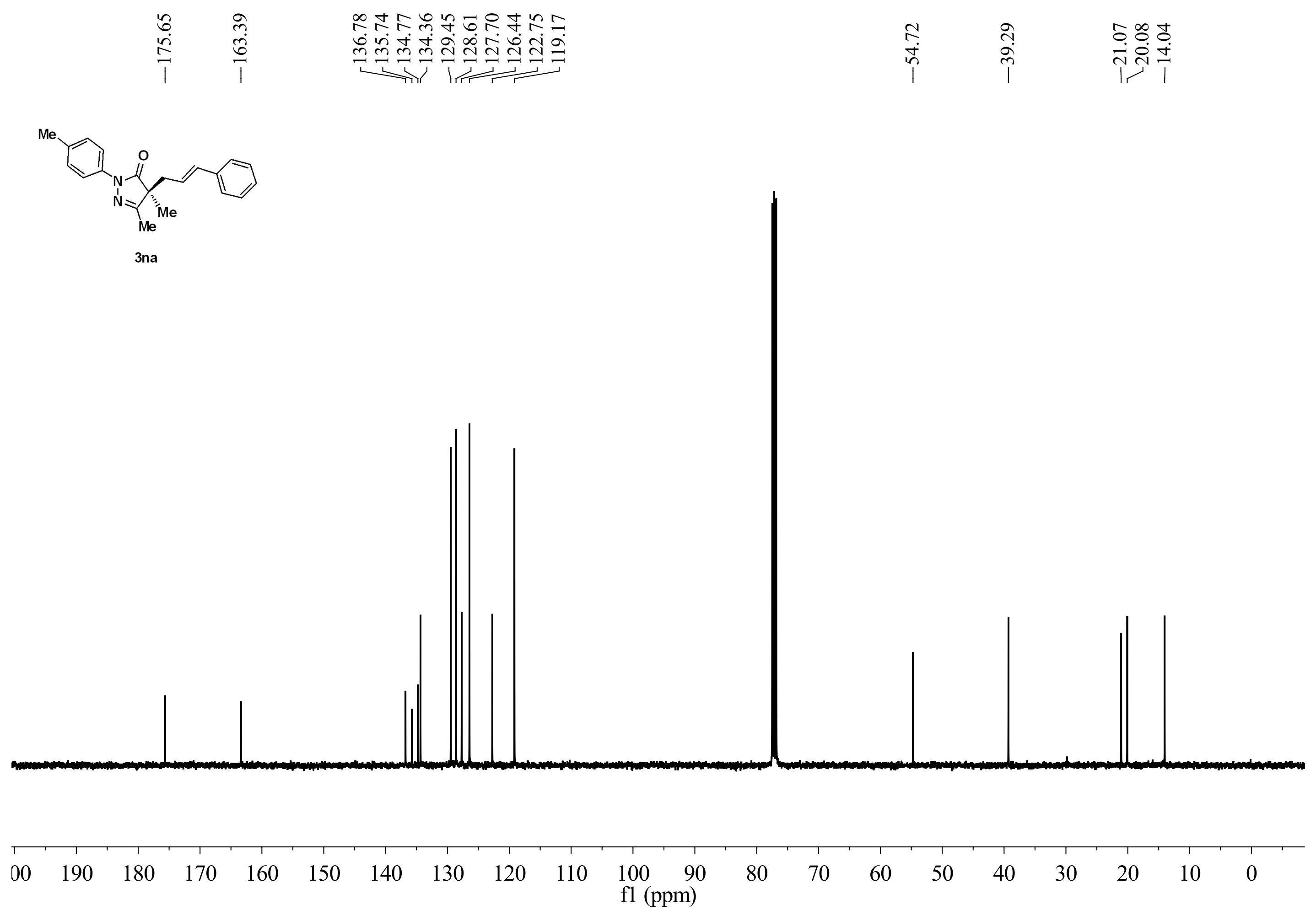


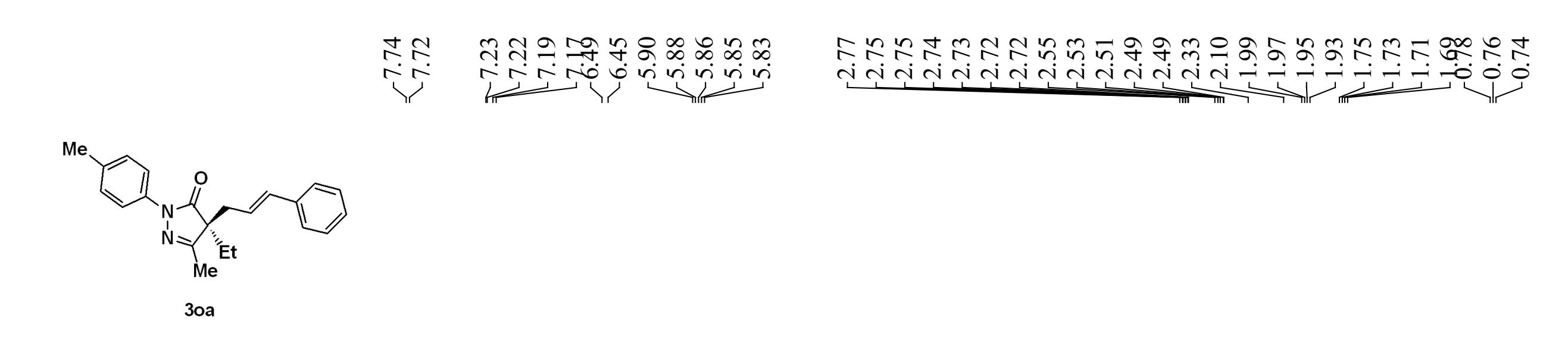



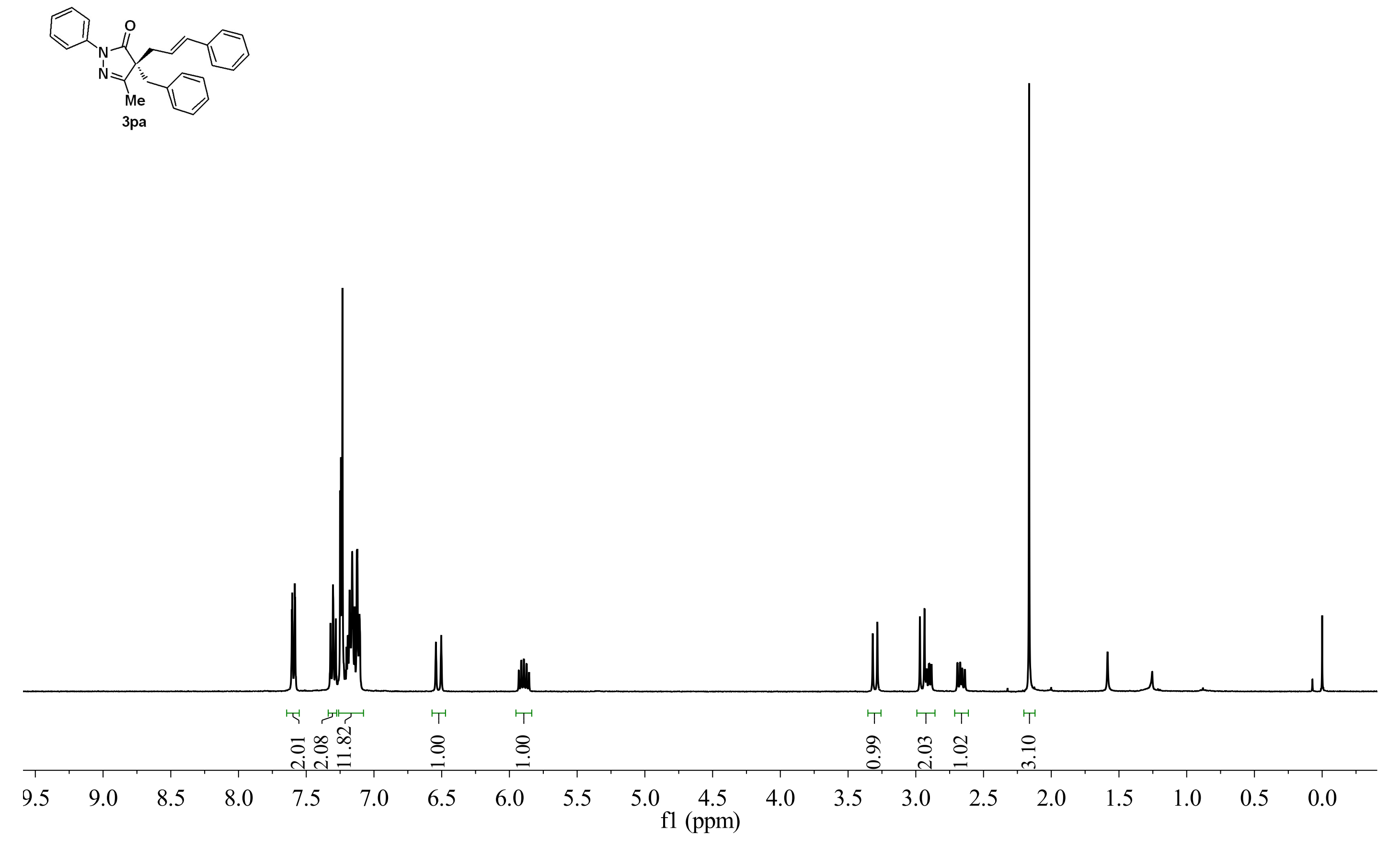


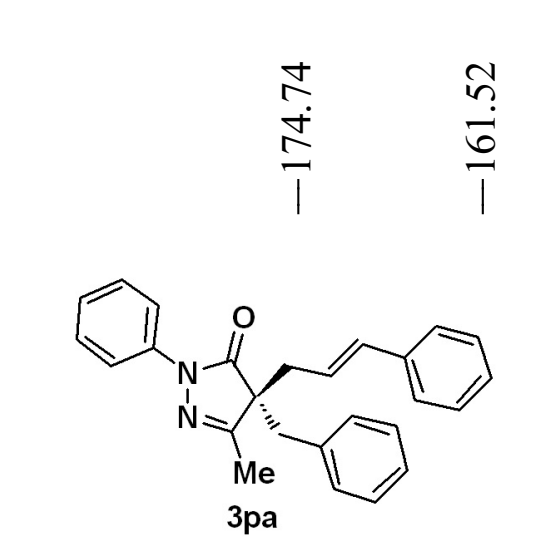




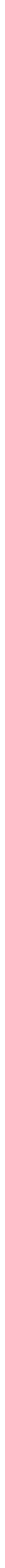



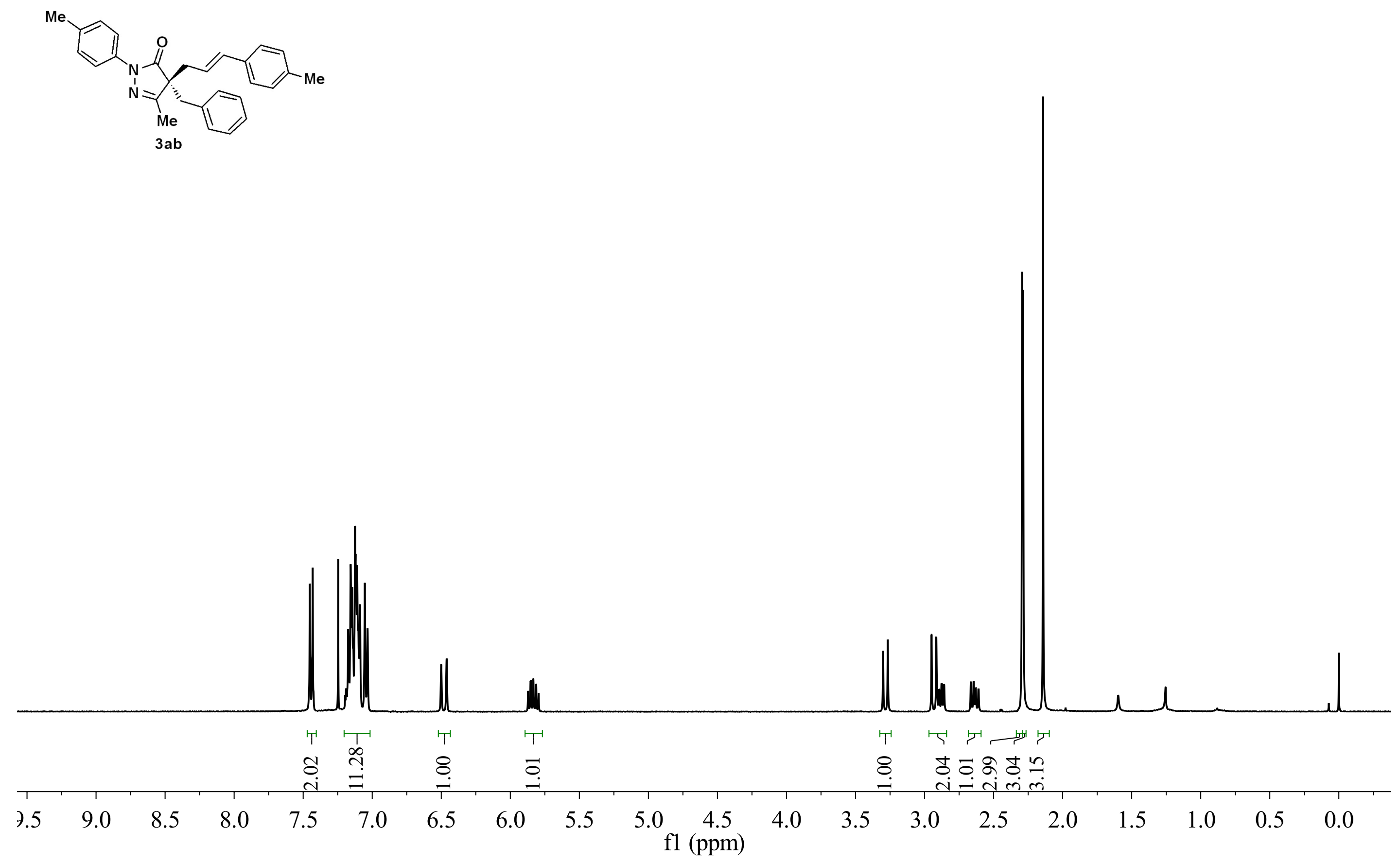


$$
\text { Ll }
$$




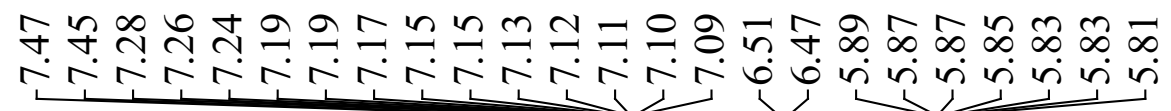

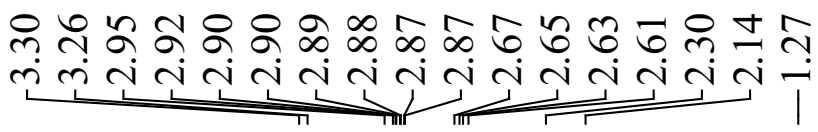
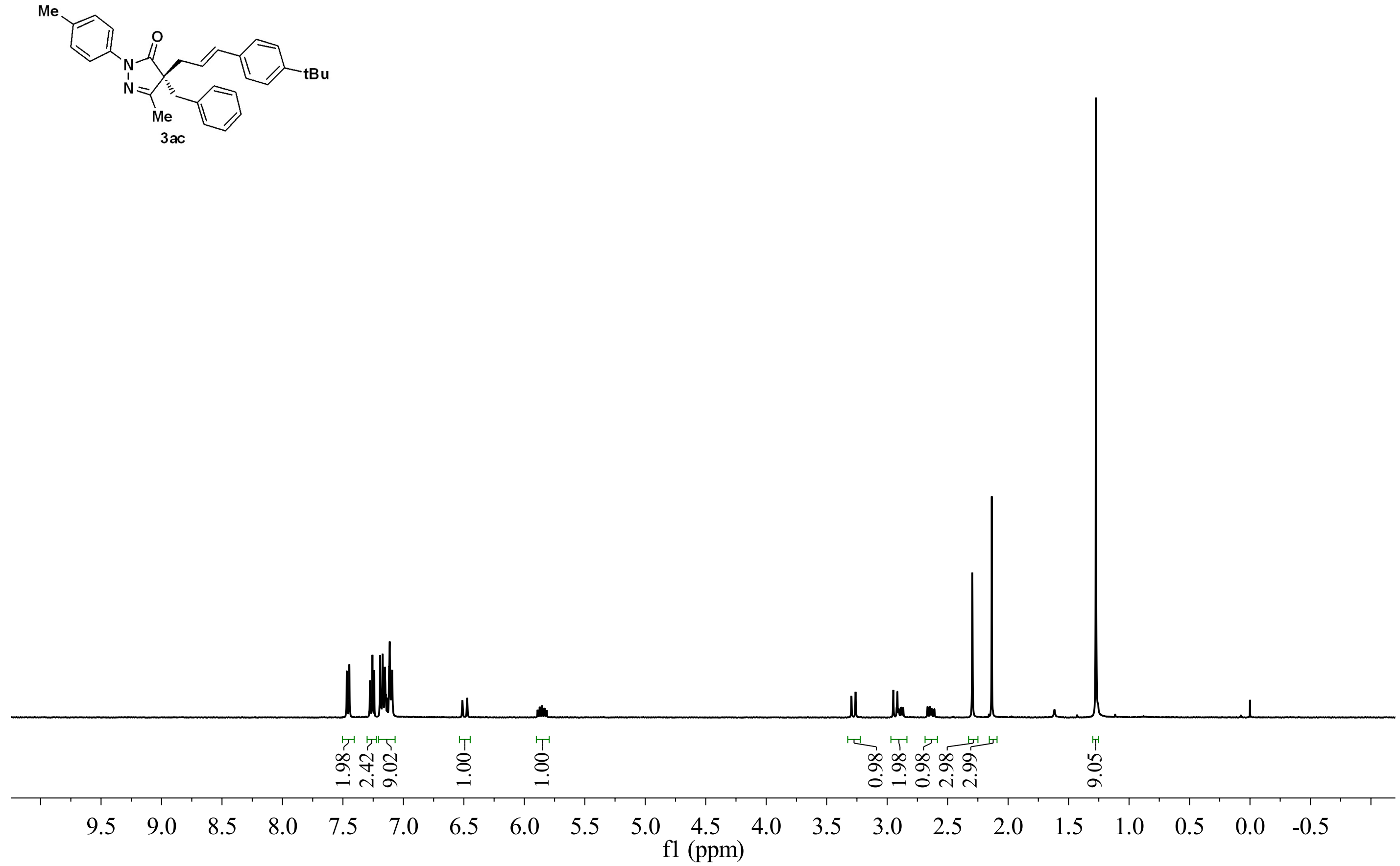


$$
\text { لـ }
$$



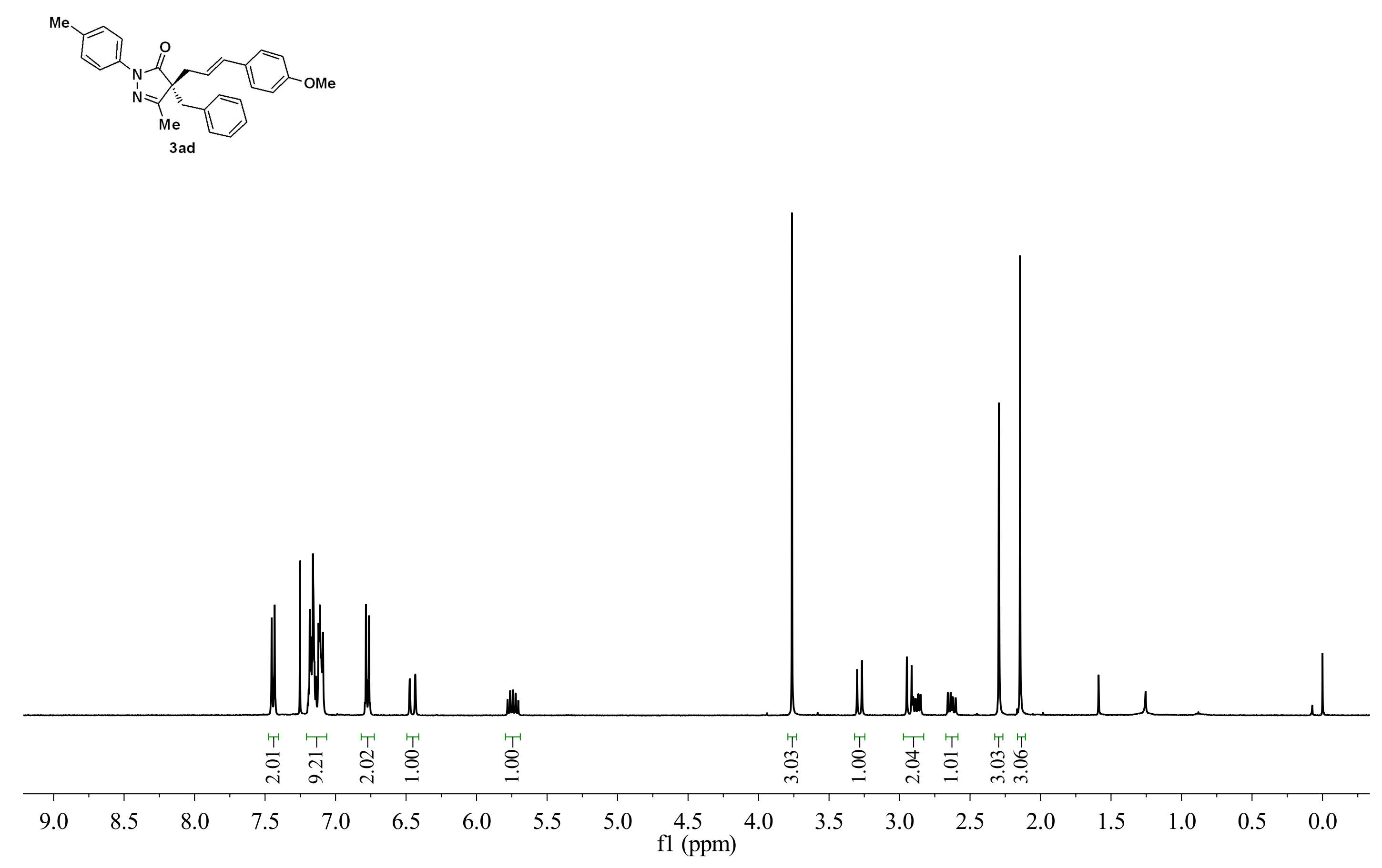


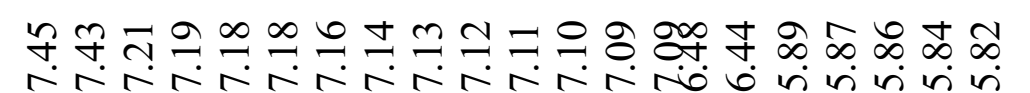

चत्र
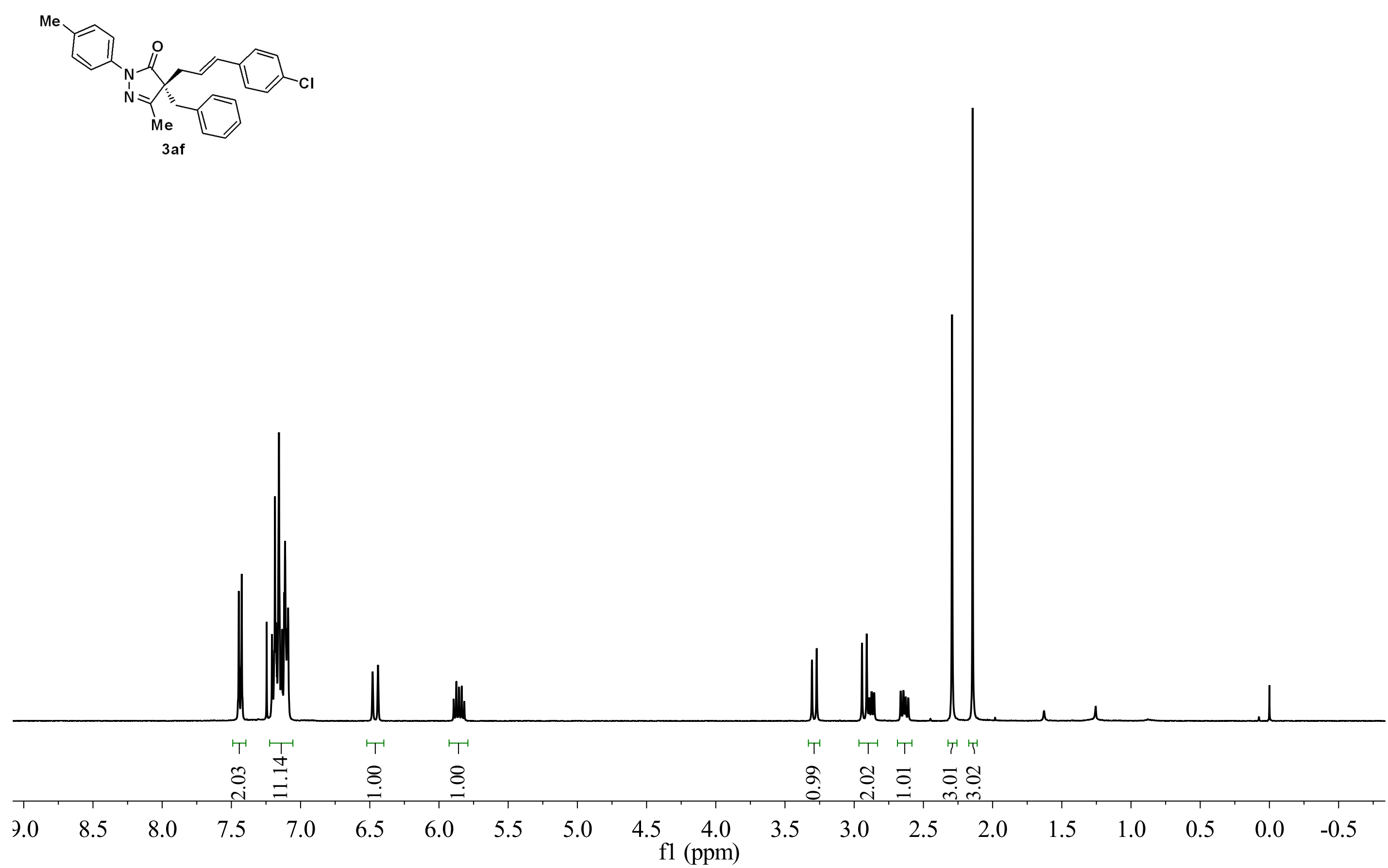
$\mathrm{Me}$
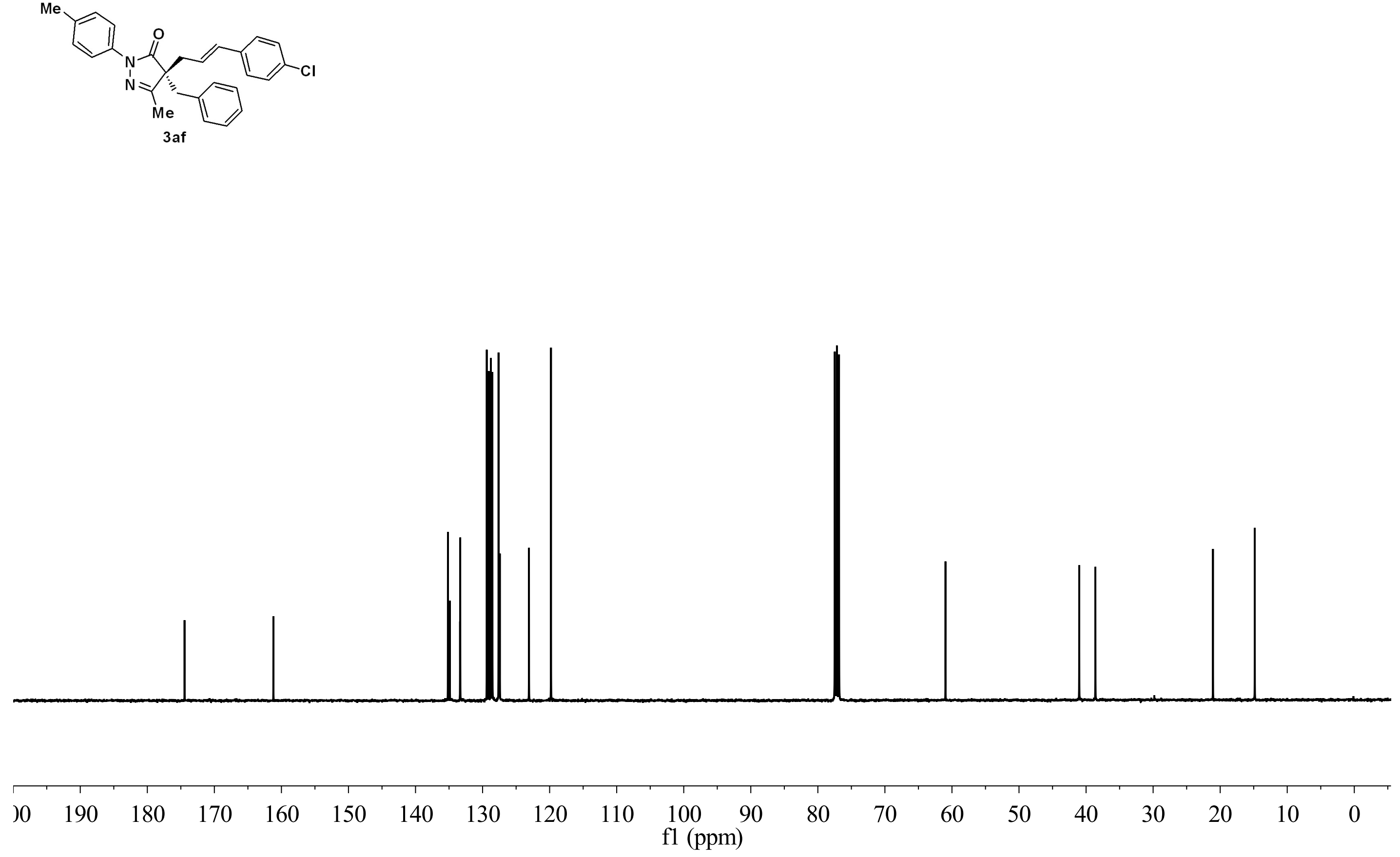


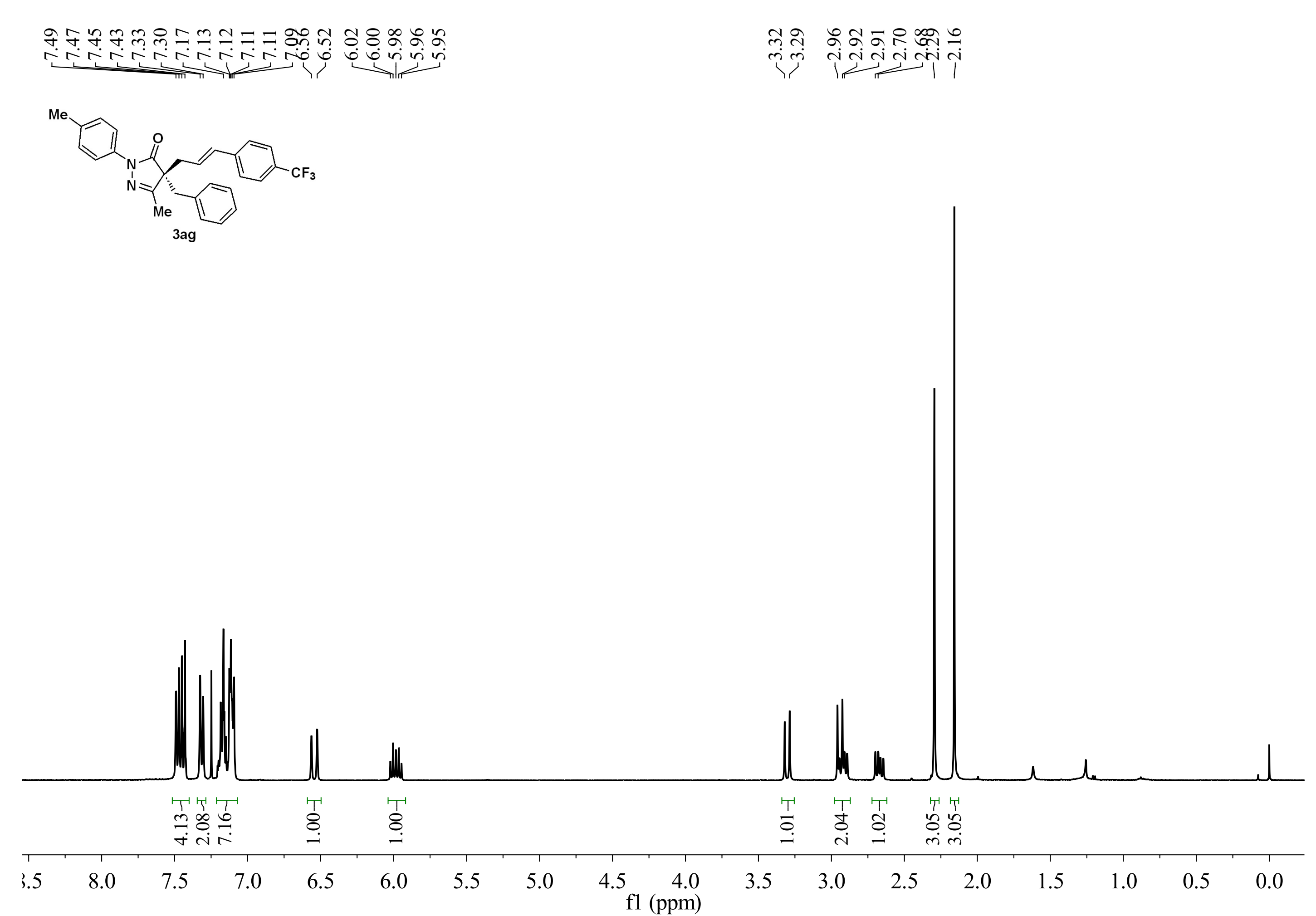




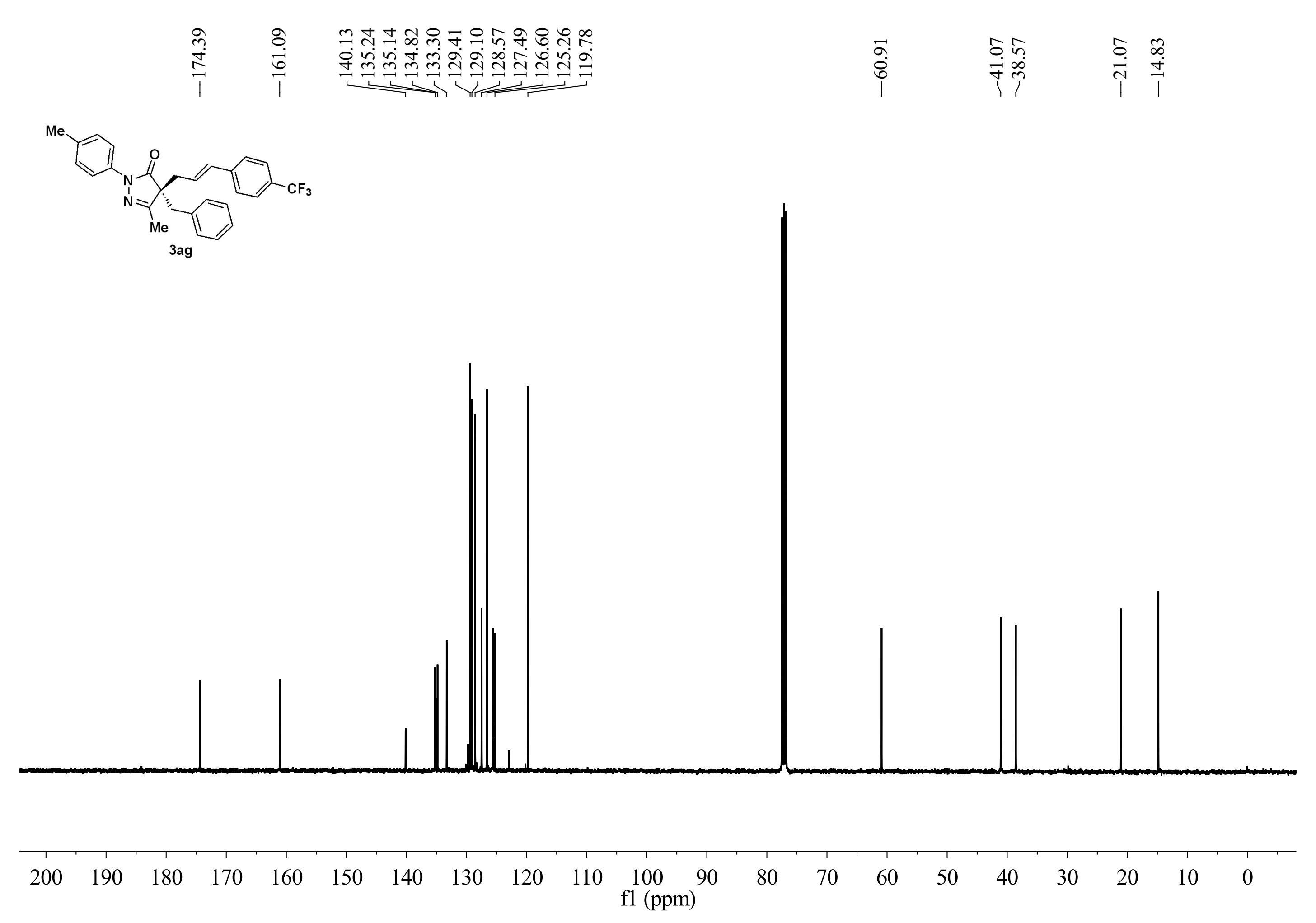



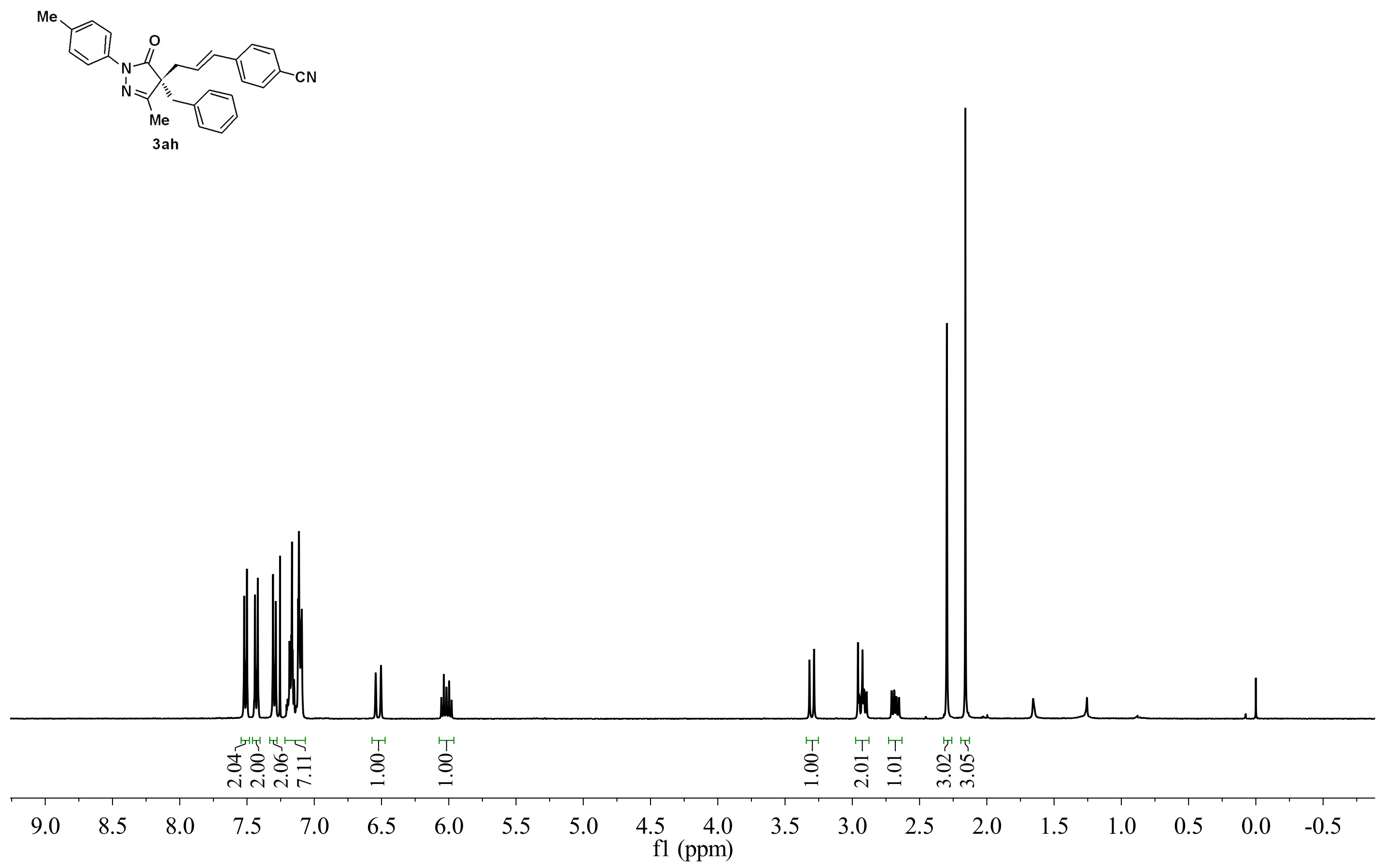


$$
\text { al }
$$




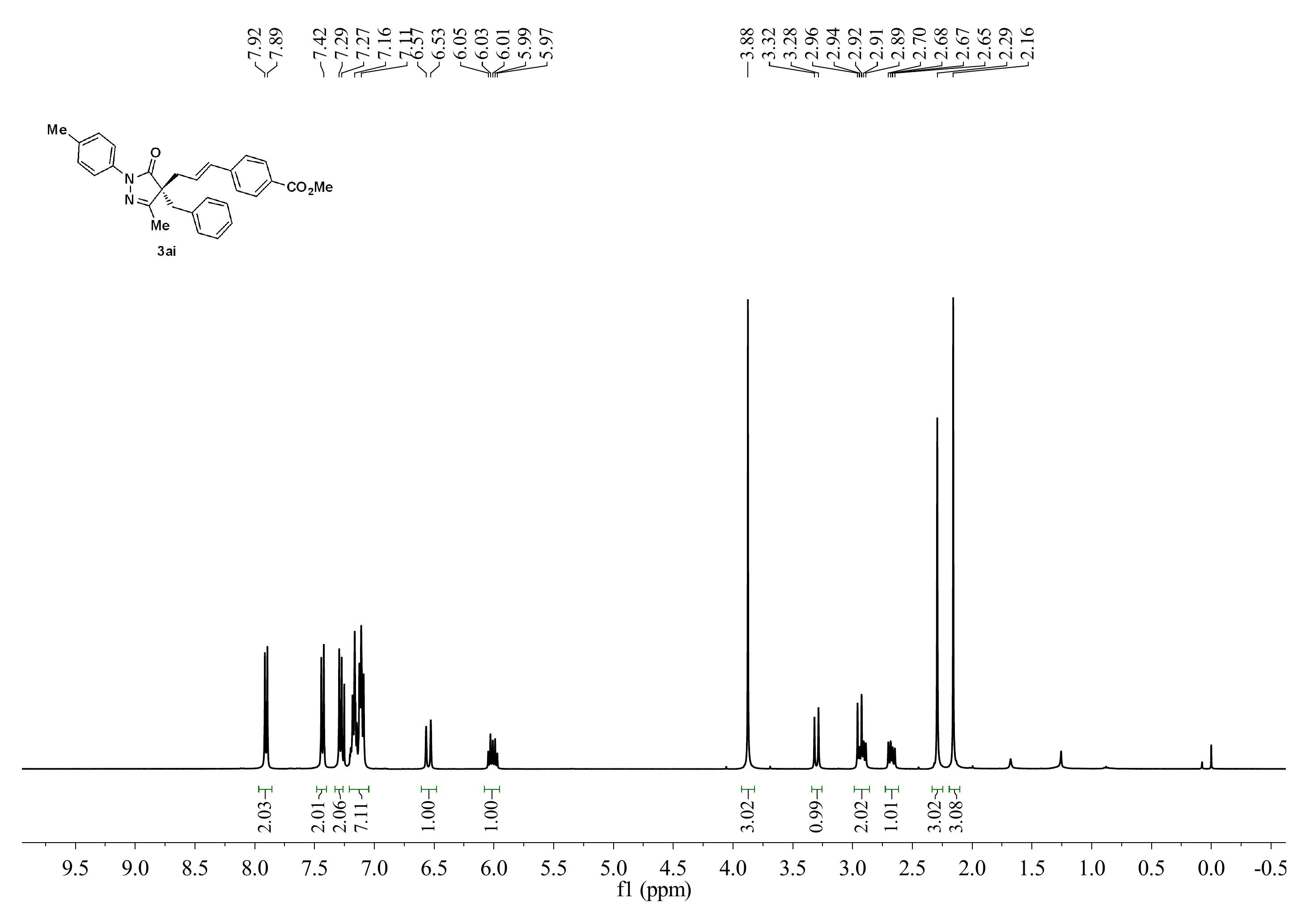




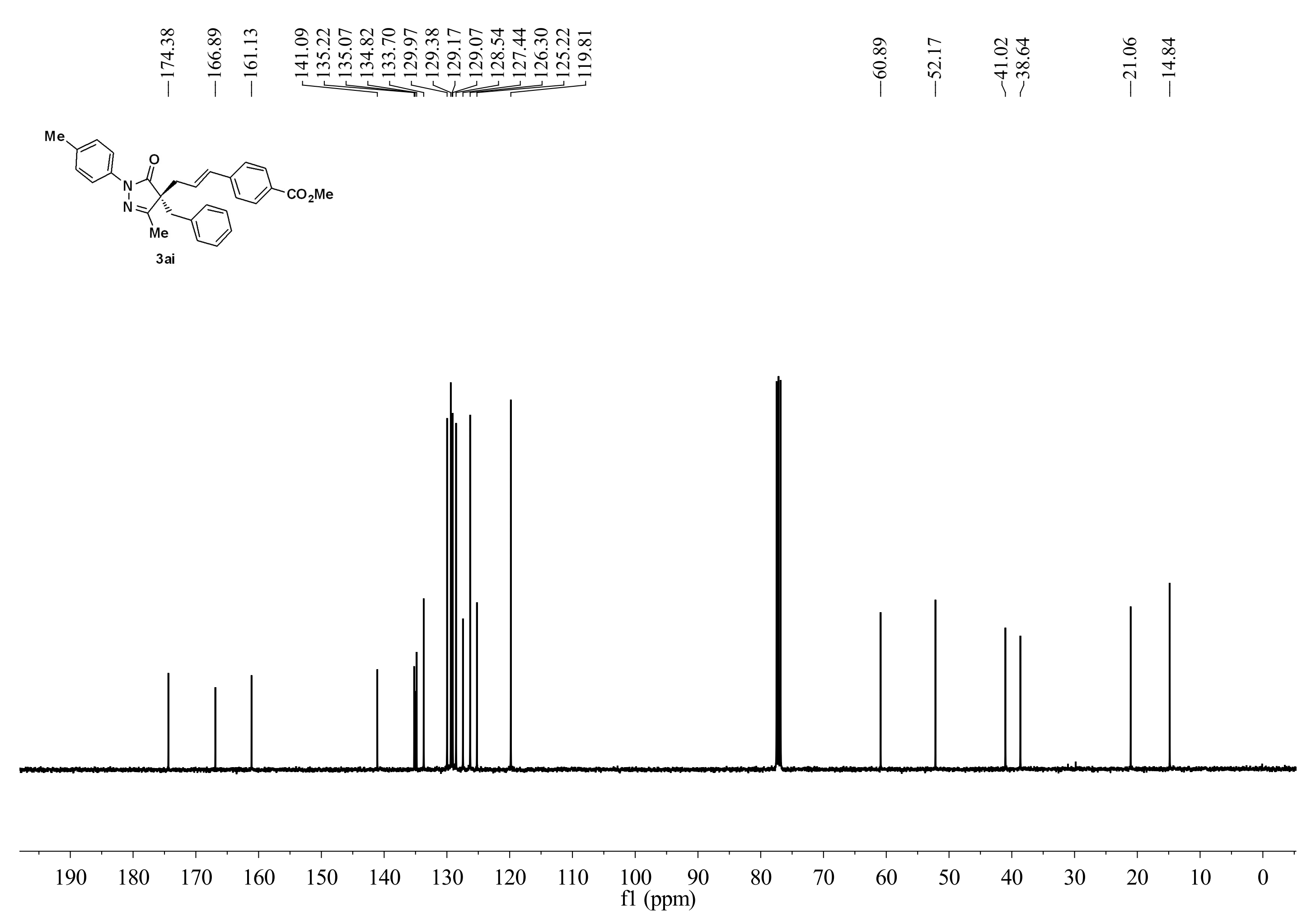



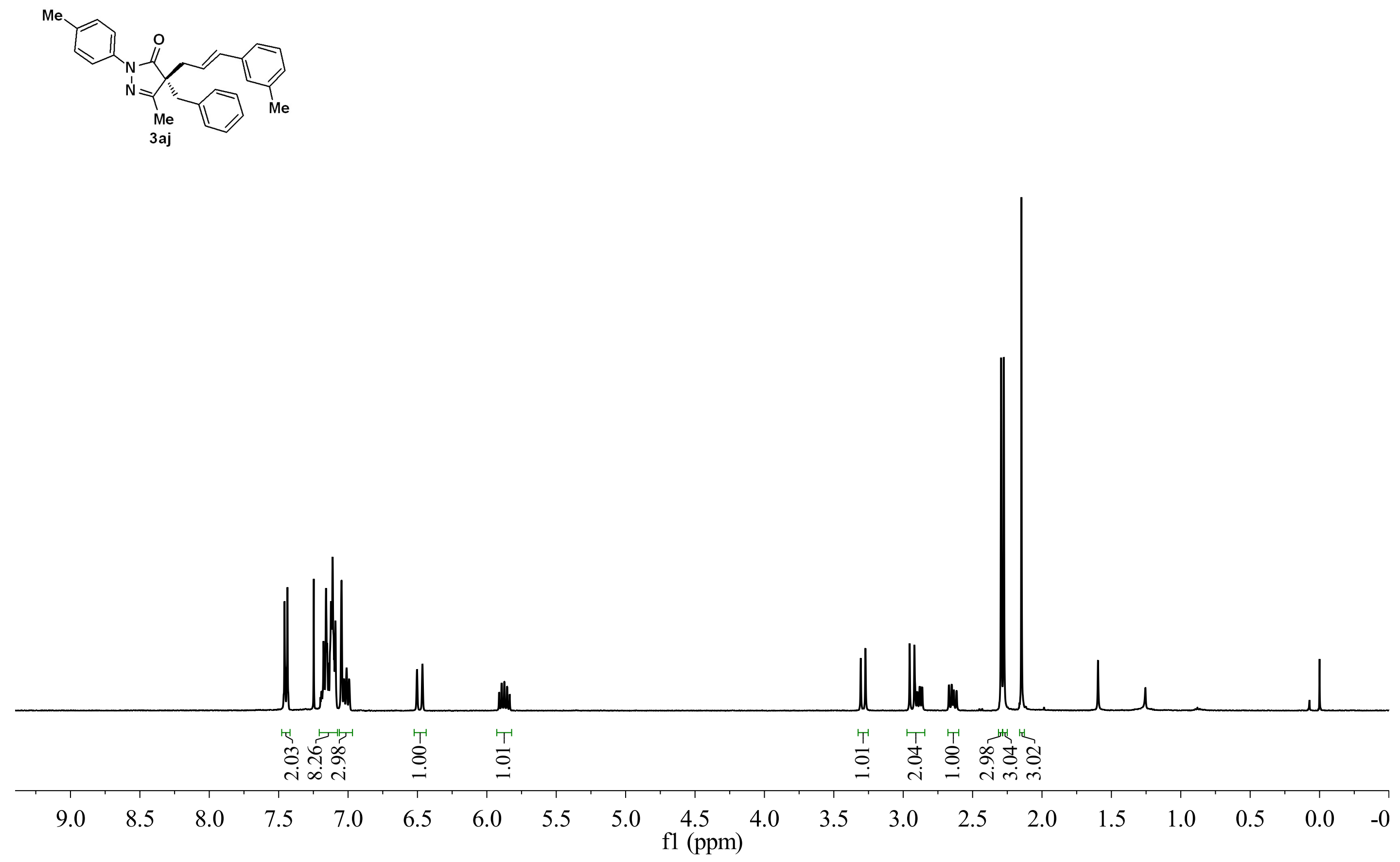


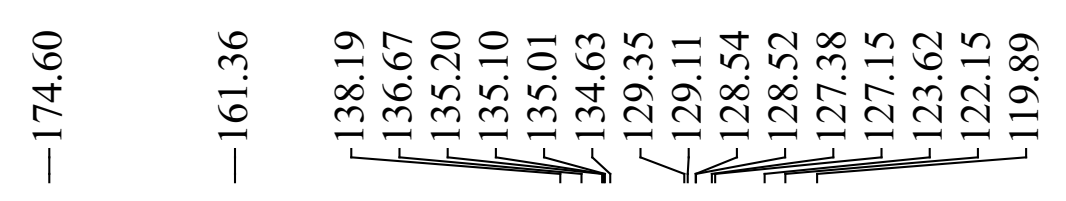

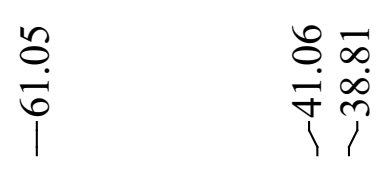

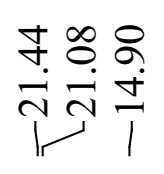
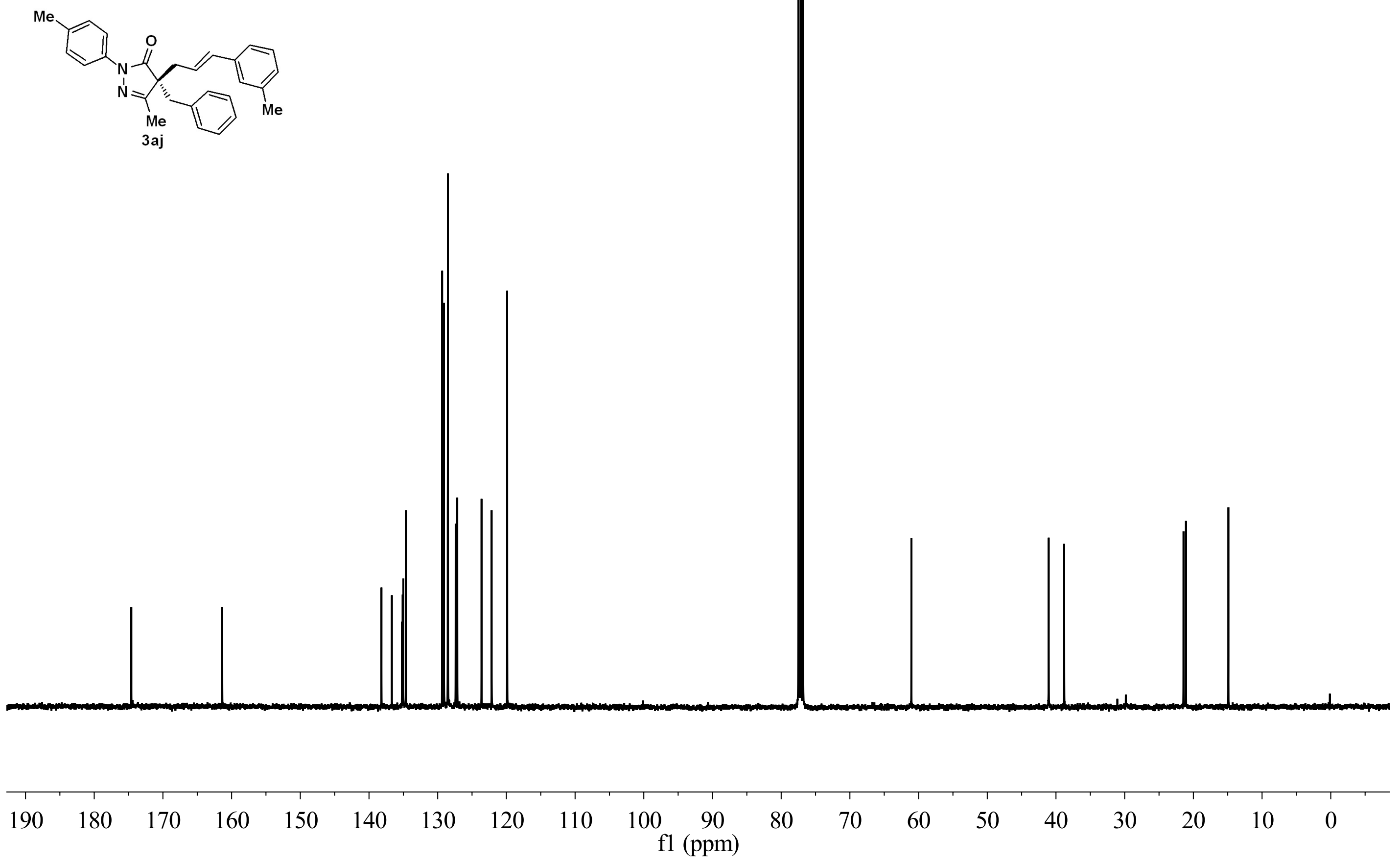


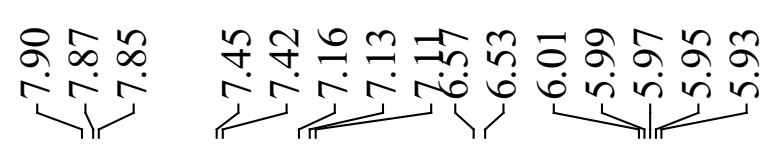
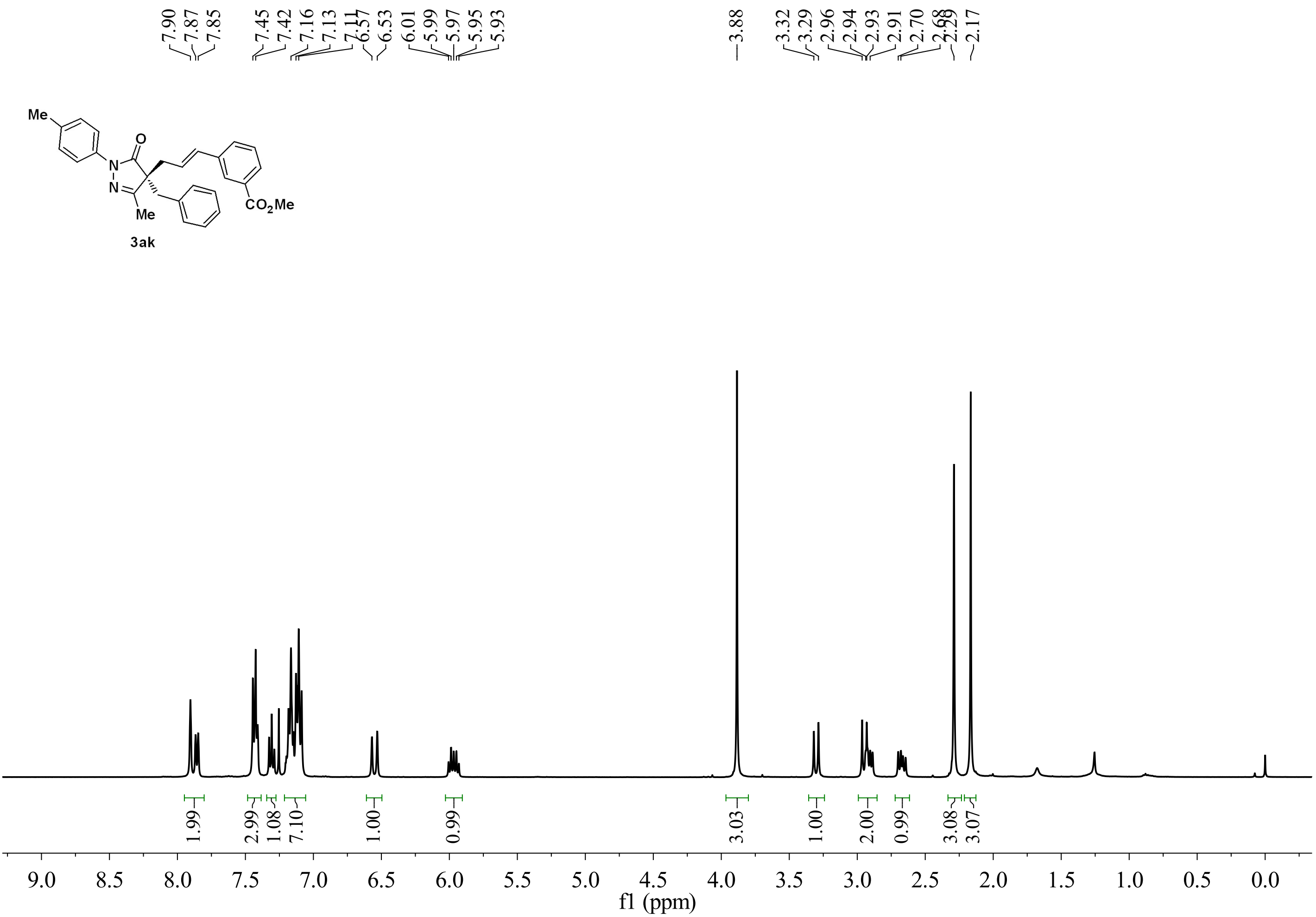


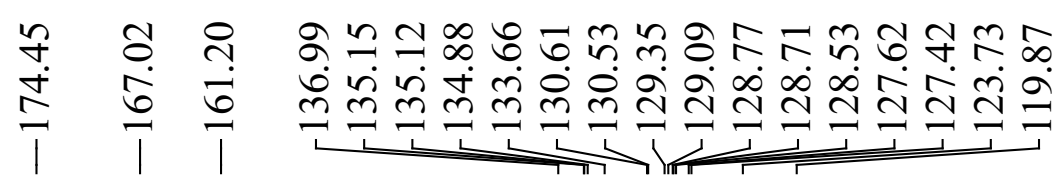

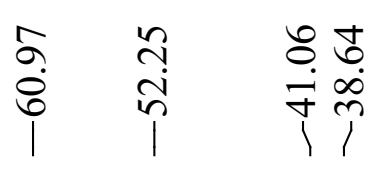

$\stackrel{8}{\stackrel{+}{i}} \stackrel{\infty}{\stackrel{+}{+}}$
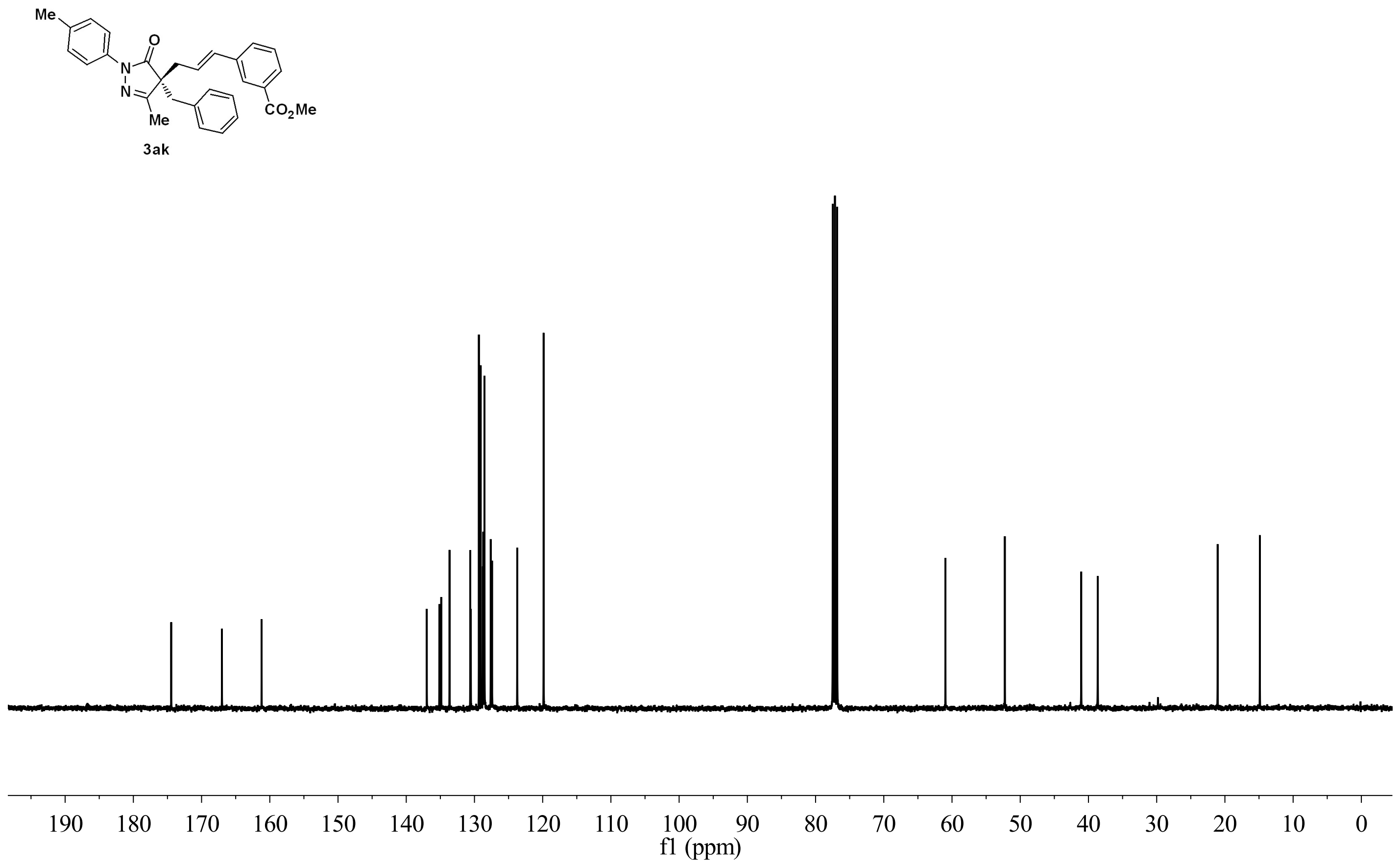


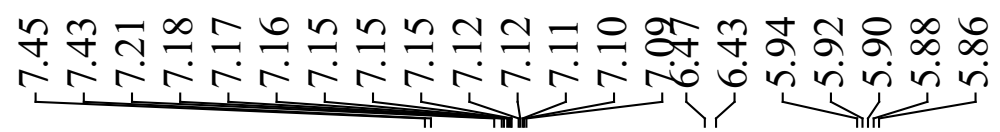

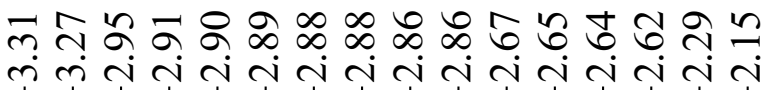
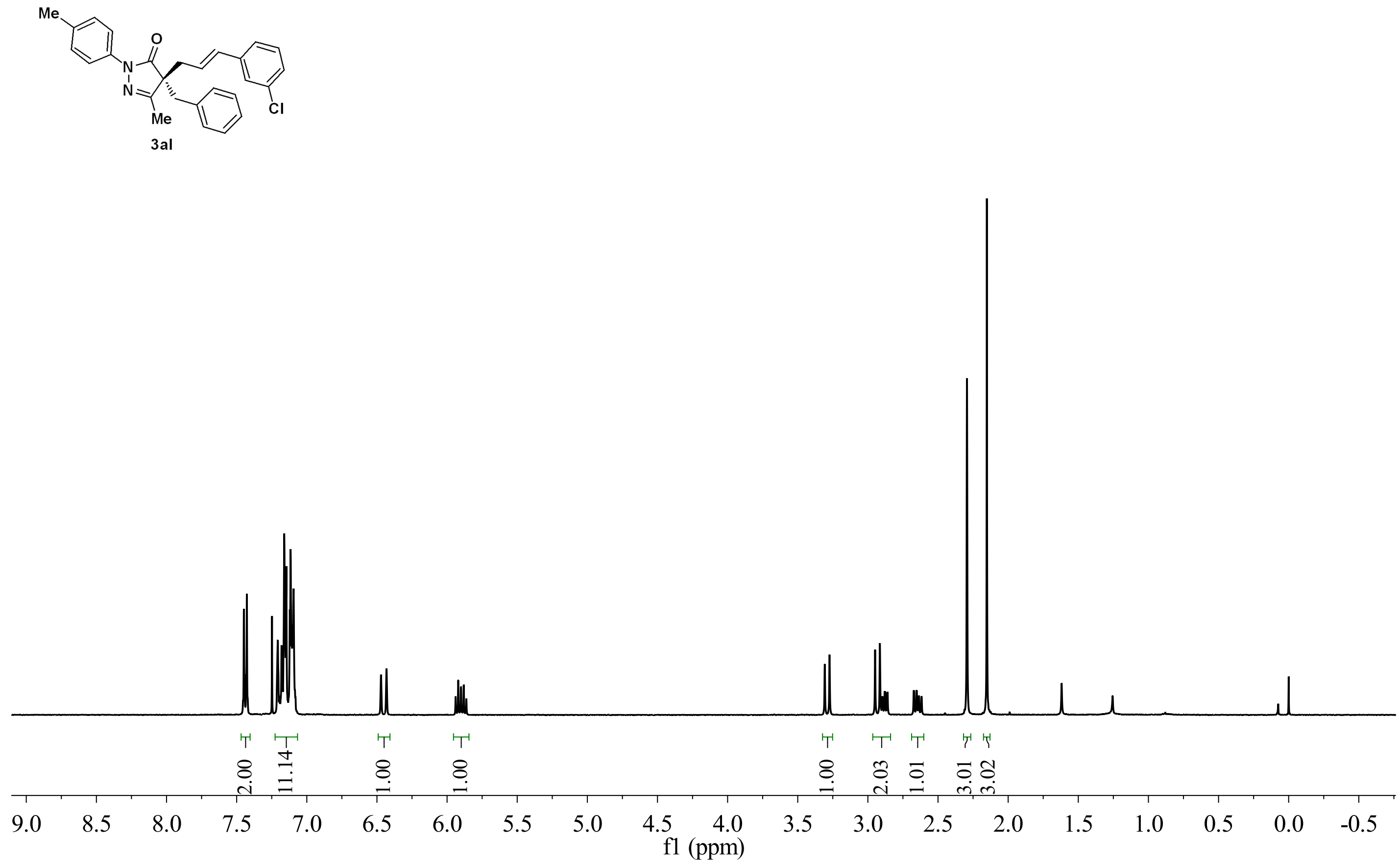


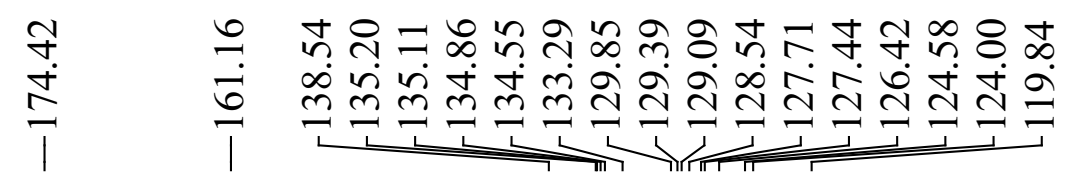

ลิ

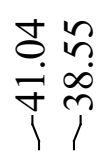

\begin{tabular}{ll}
$\infty$ & 0 \\
\hdashline & $\infty$ \\
$i$ & $\dot{ \pm}$
\end{tabular}

$\mathrm{Me}$

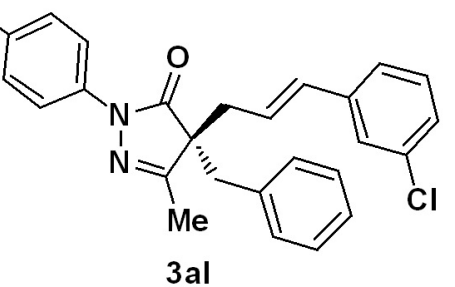

3al
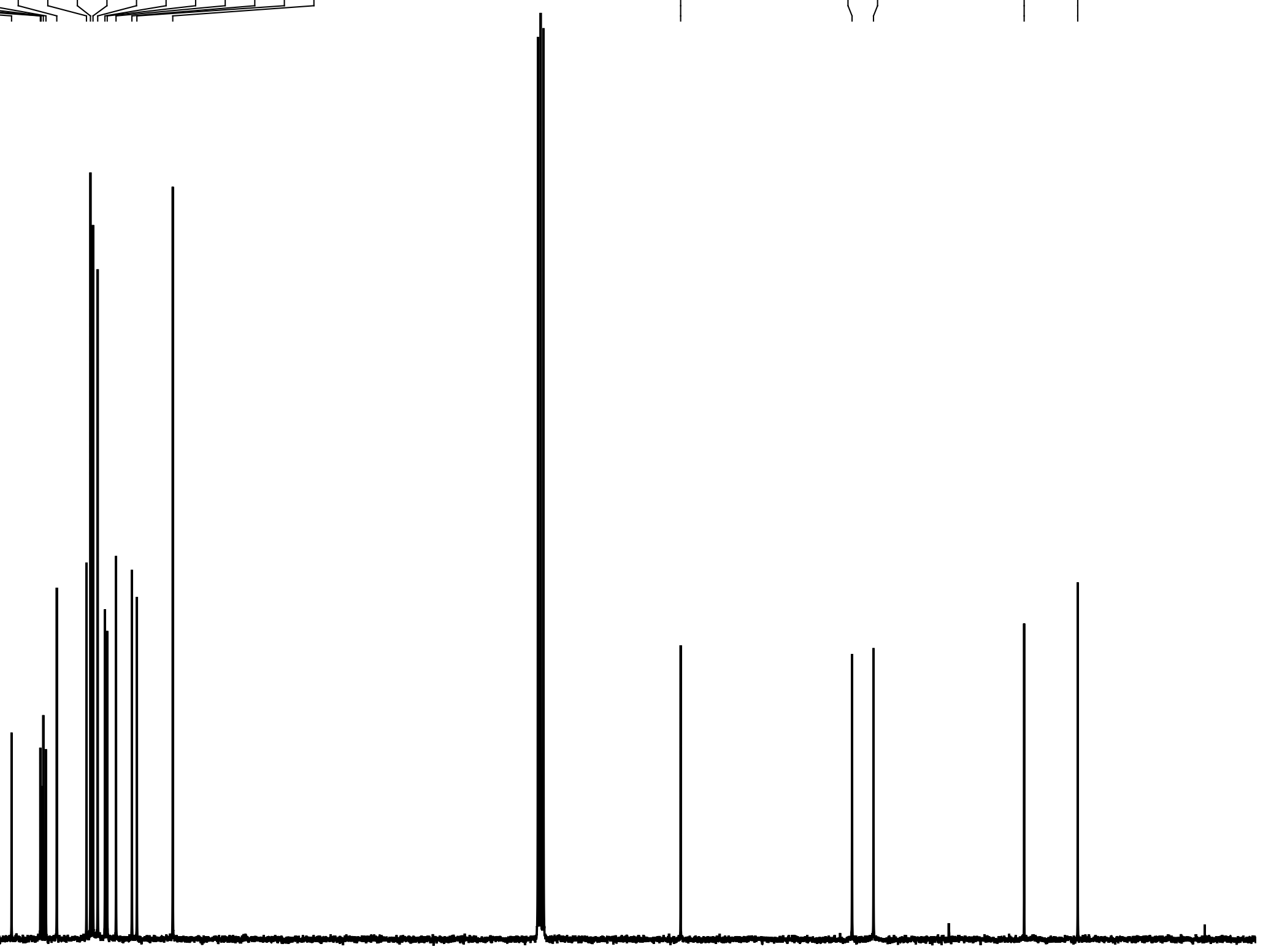

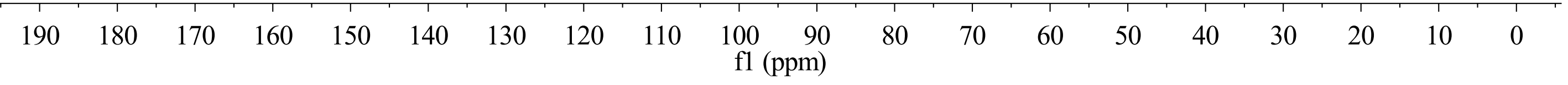




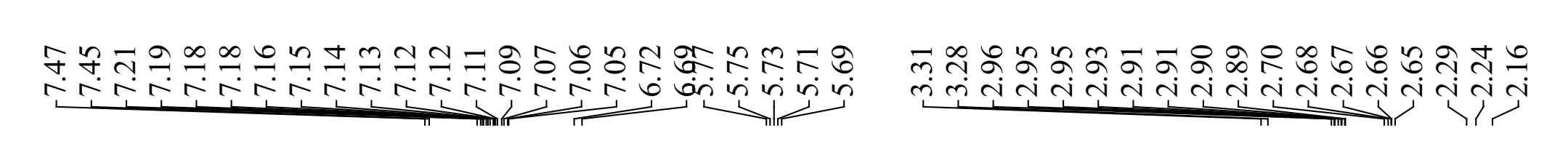

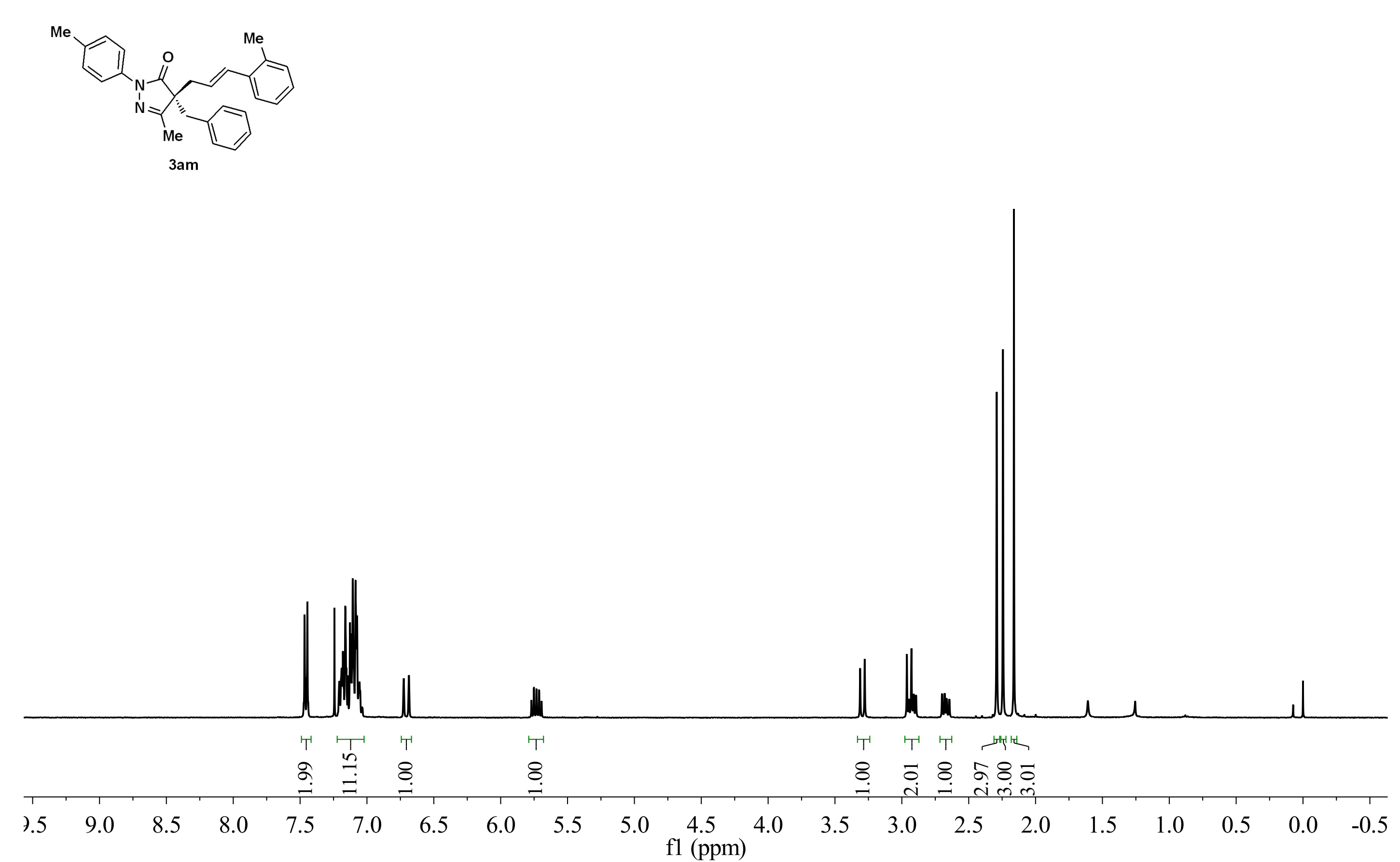




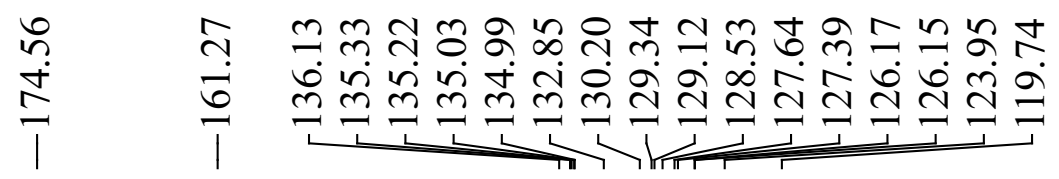

$\mathrm{Me}$
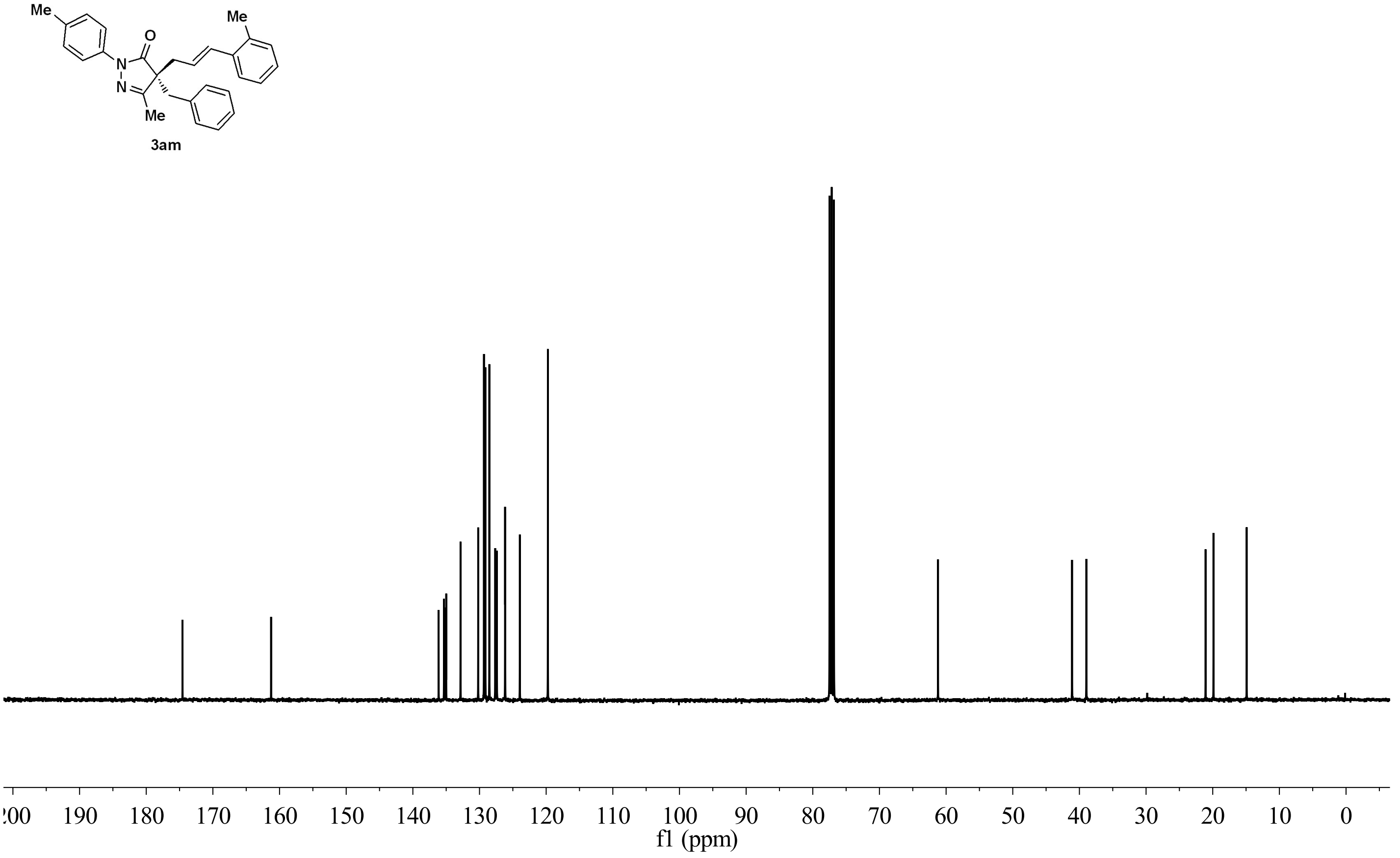


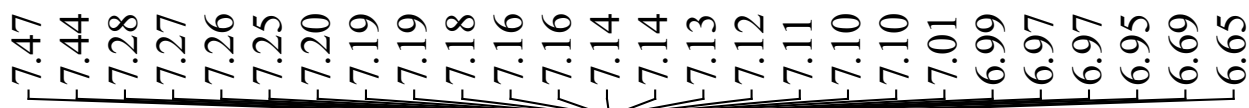

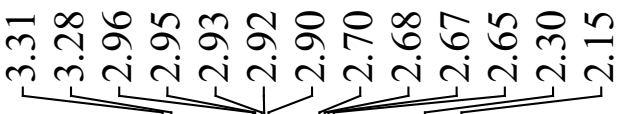
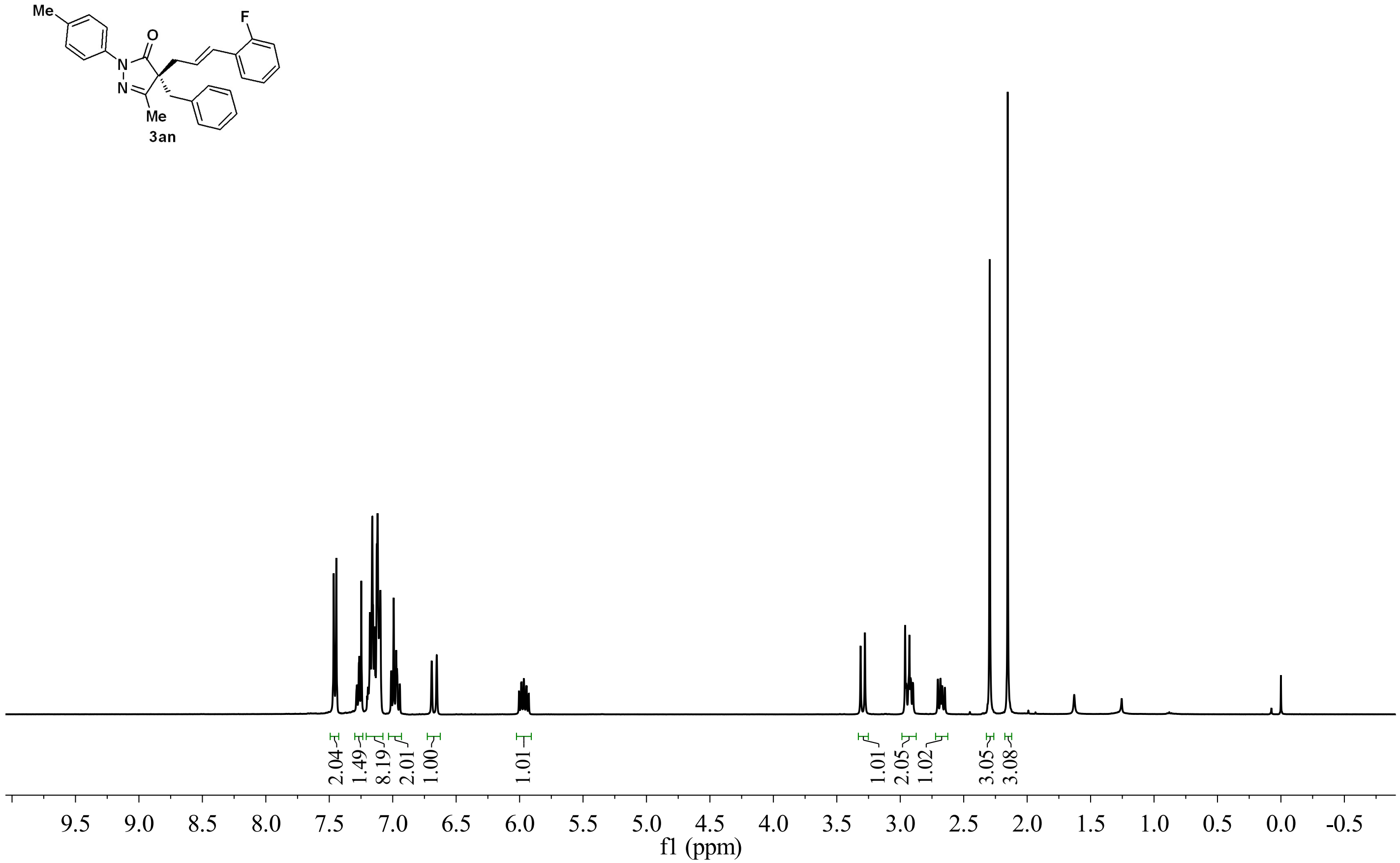


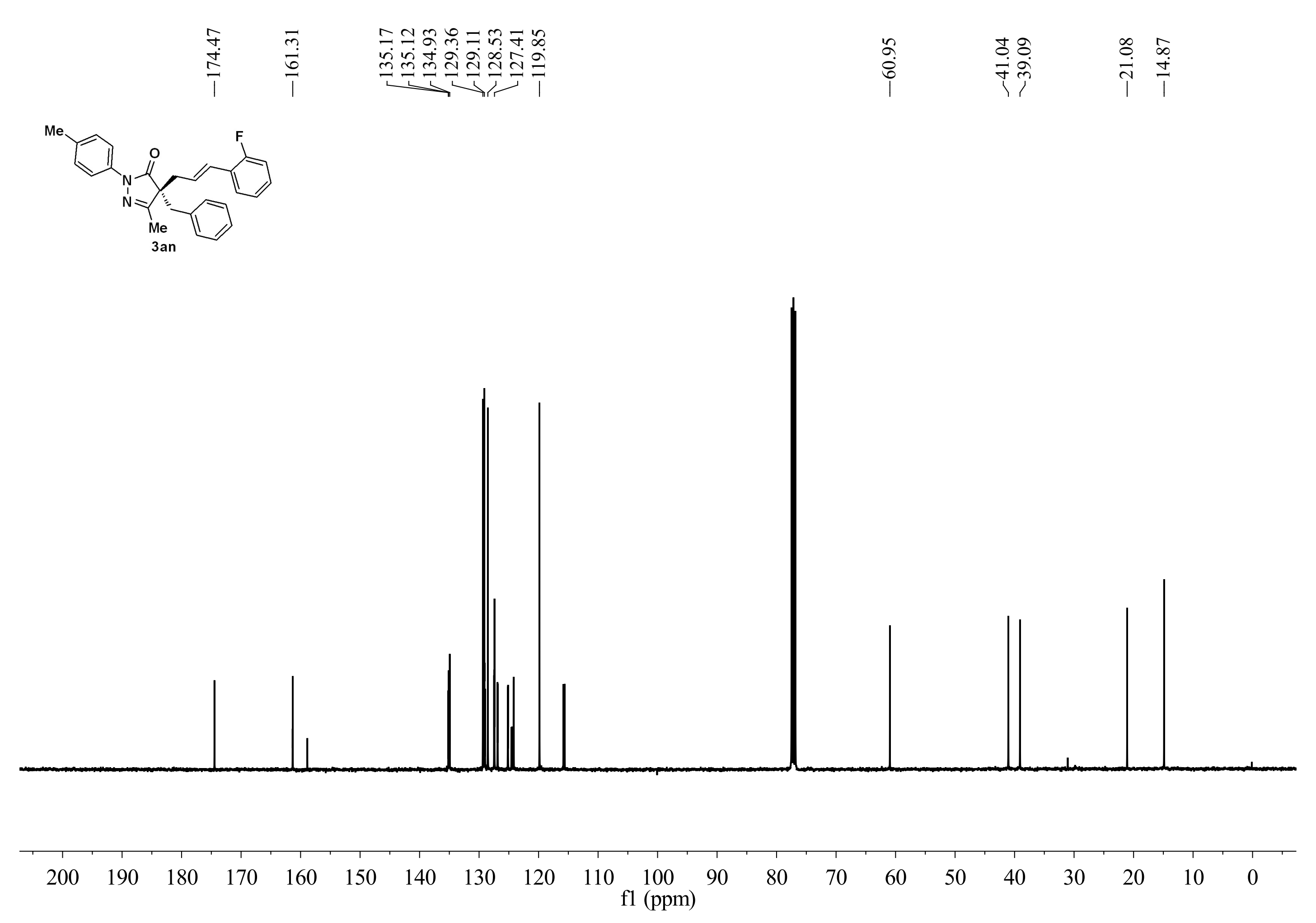



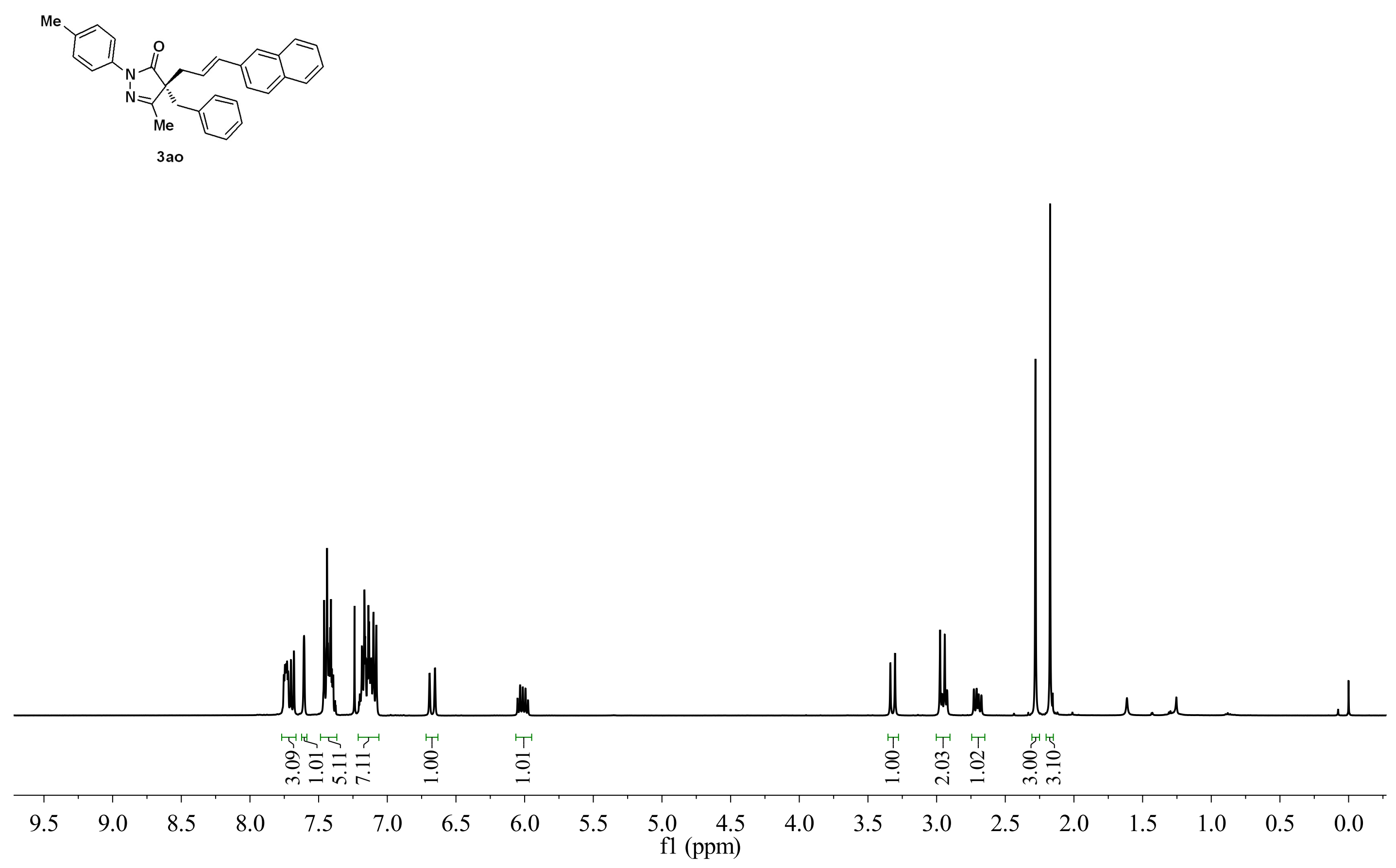


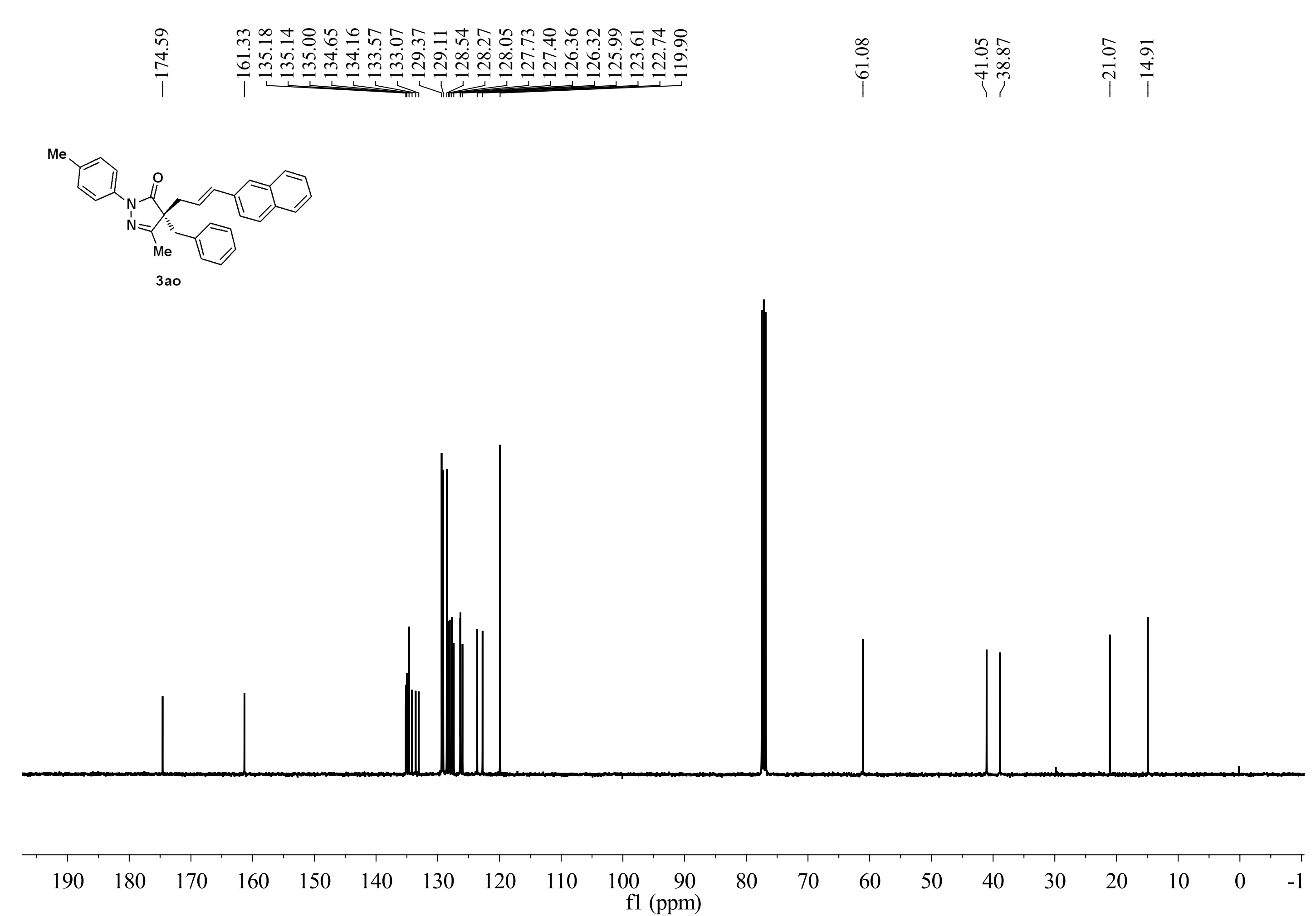




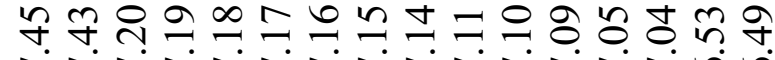

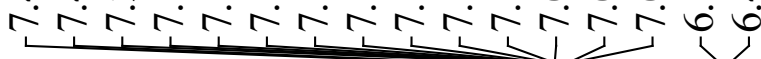
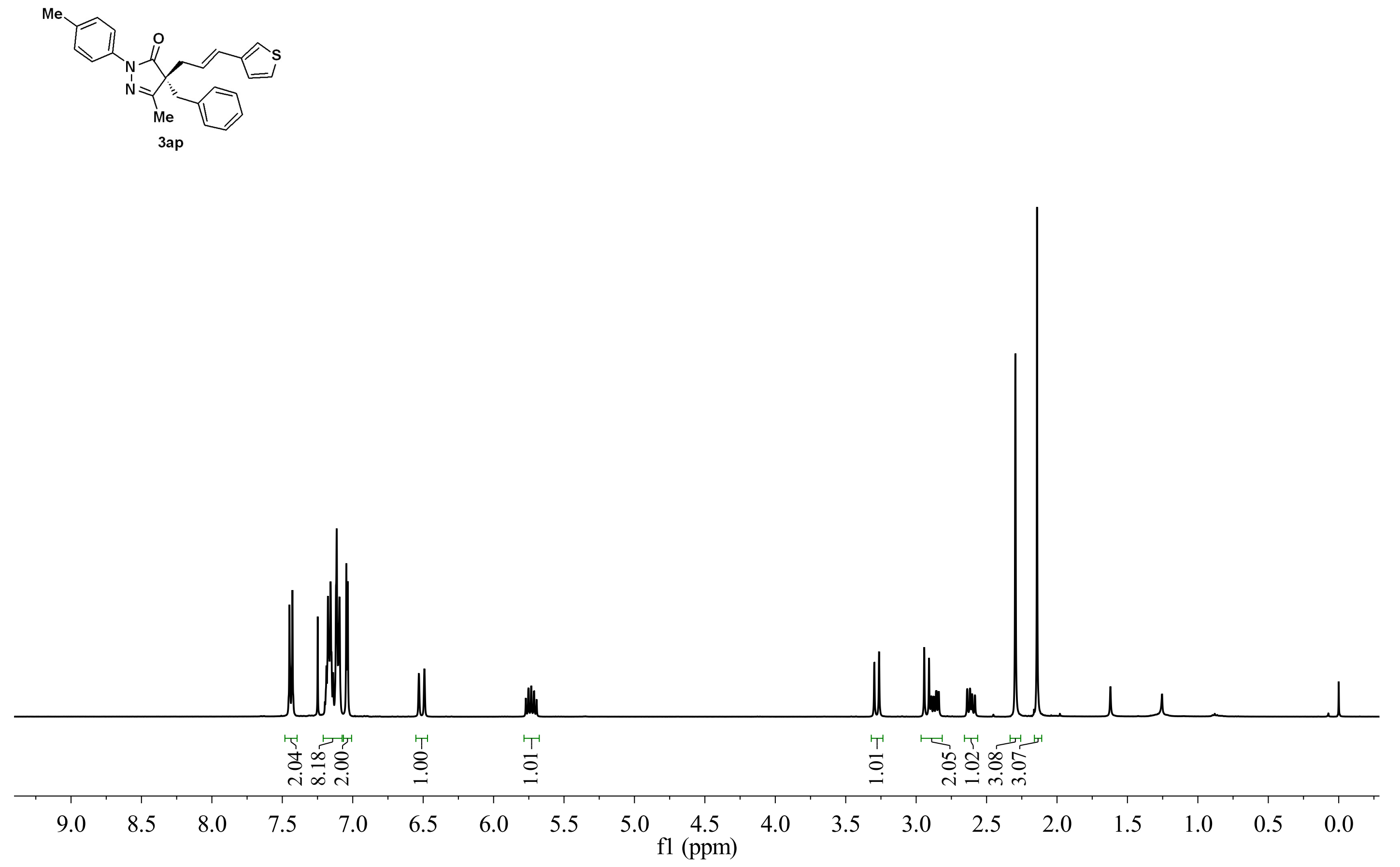


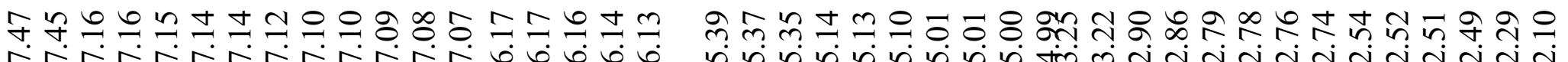

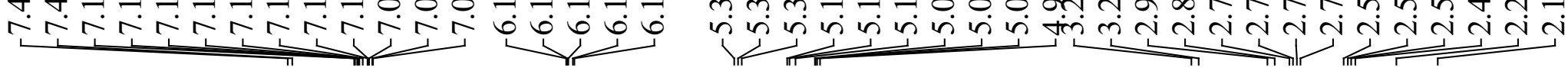<smiles>C=C/C=C/C[C@@]1(Cc2ccccc2)C(=O)N(c2ccc(C)cc2)N=C1C</smiles>

5 aa

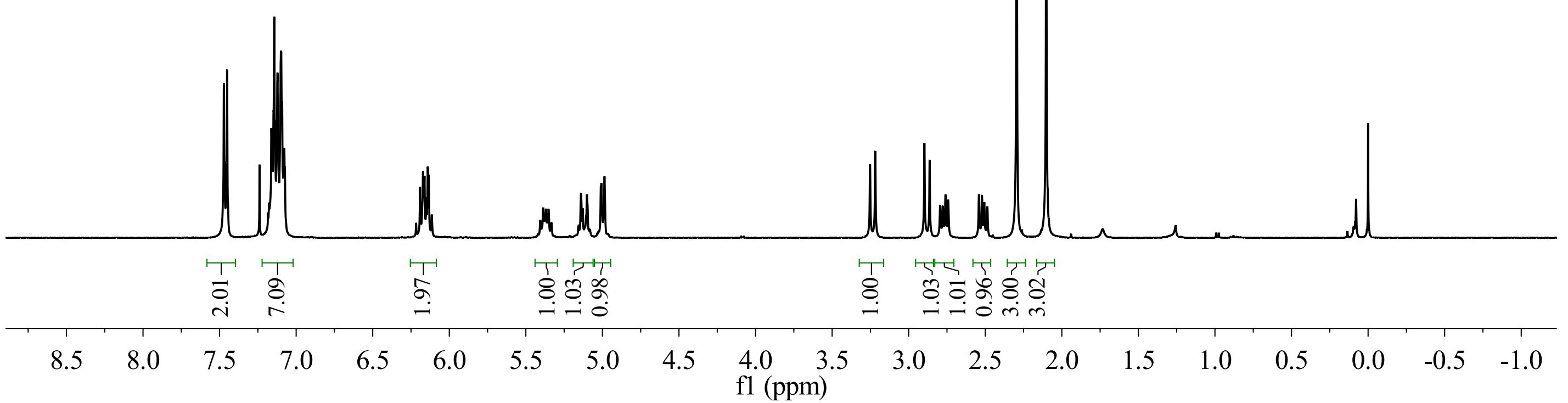


Me

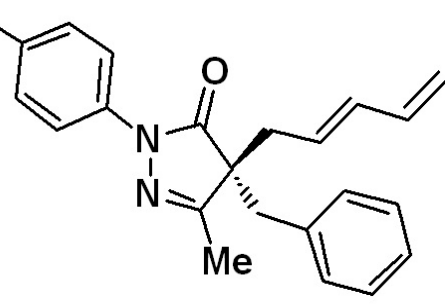

5 aa
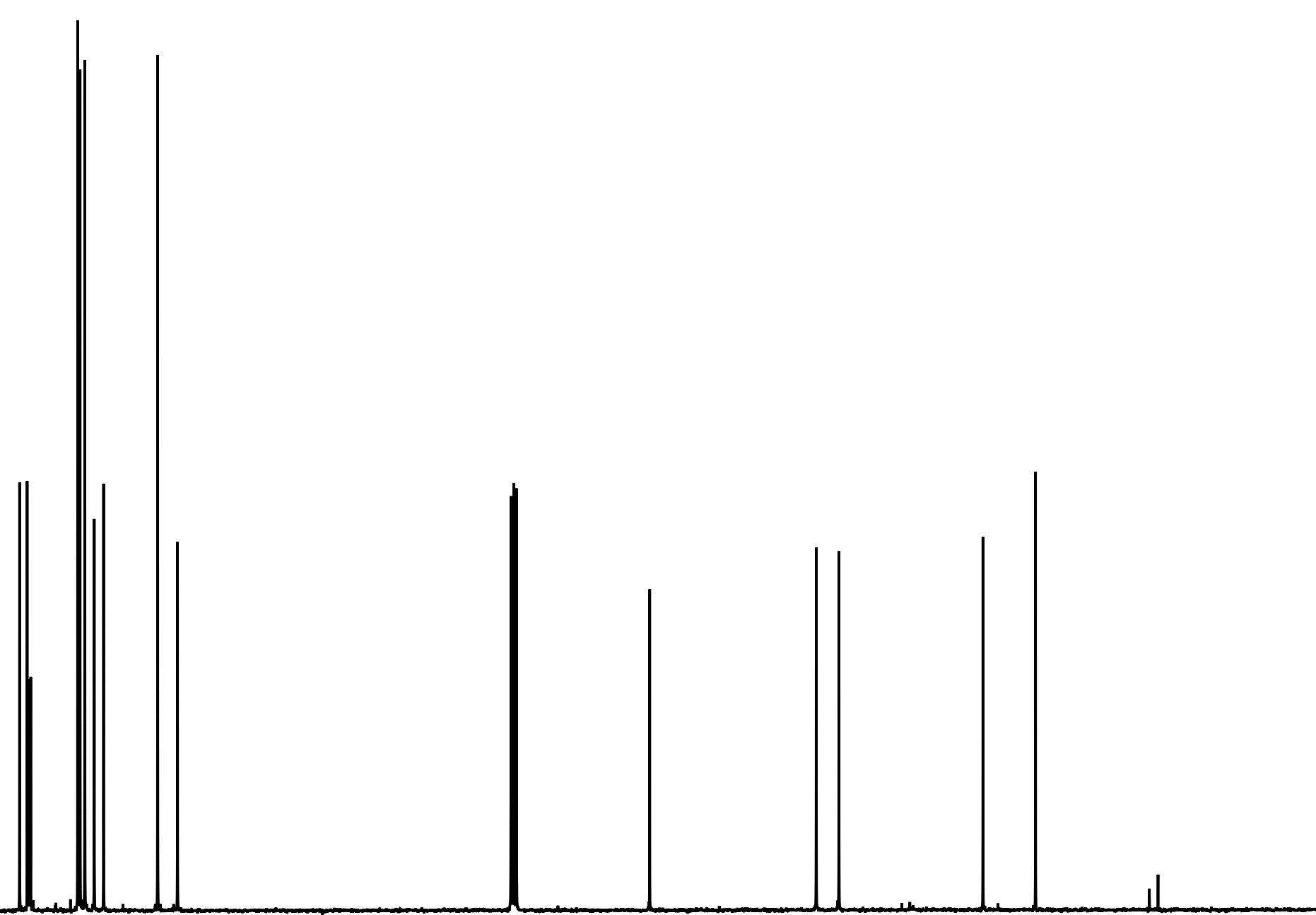
Me
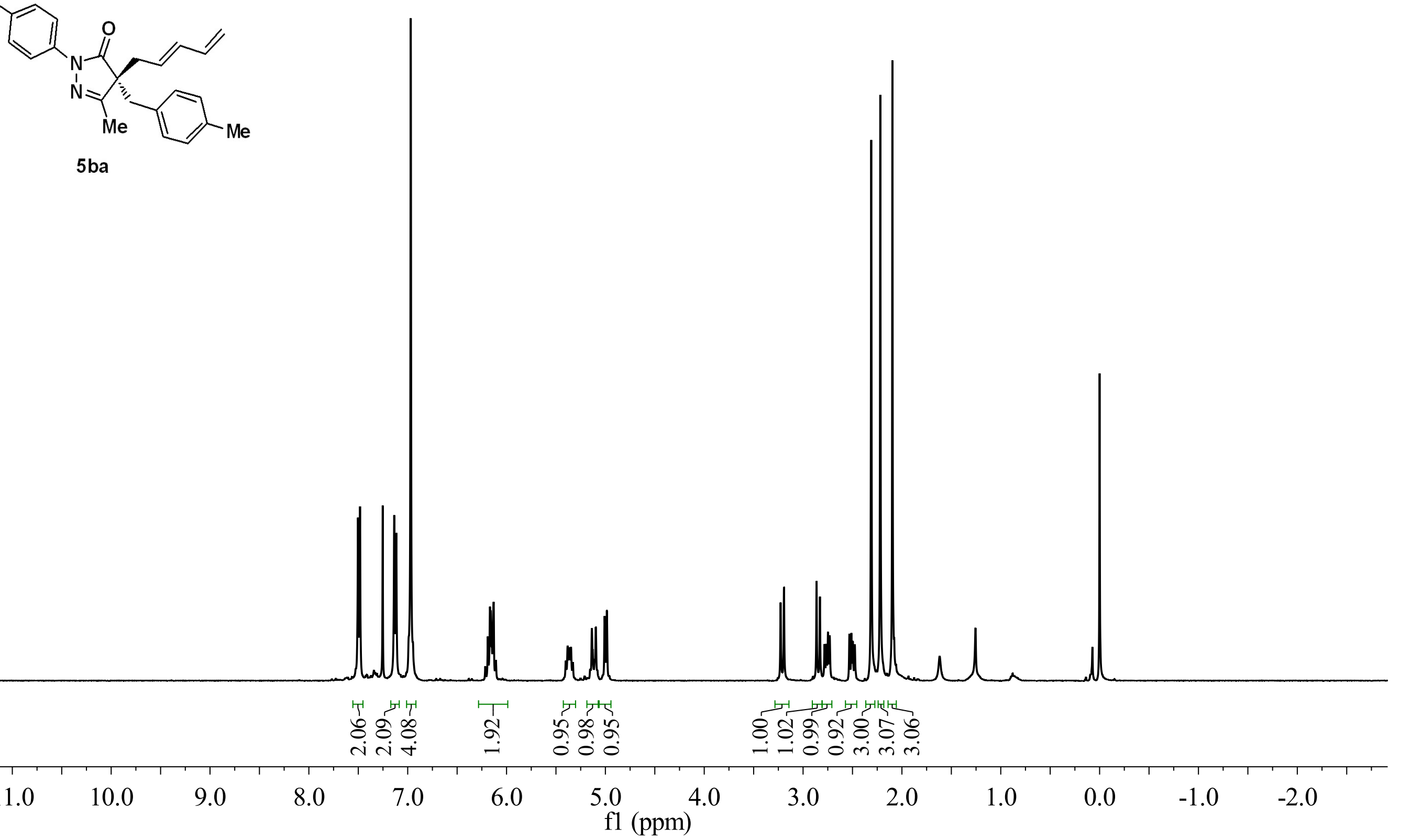


\section{昱 絜}

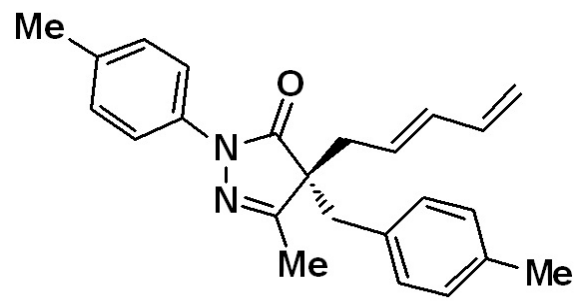

$5 \mathrm{ba}$

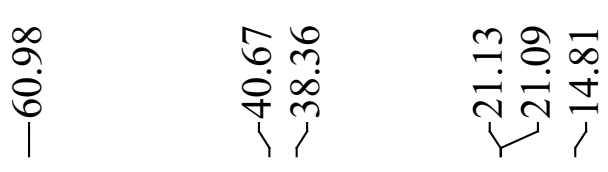

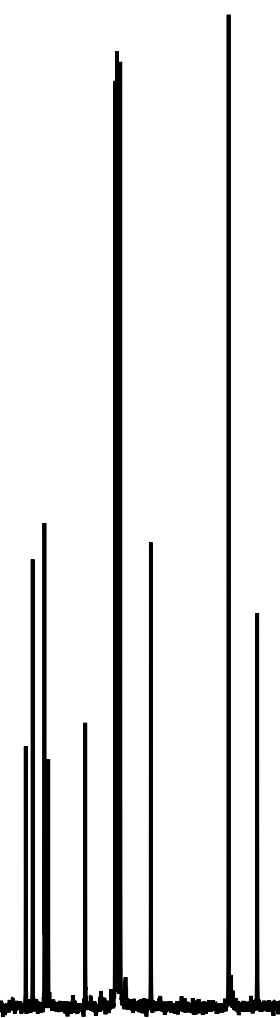




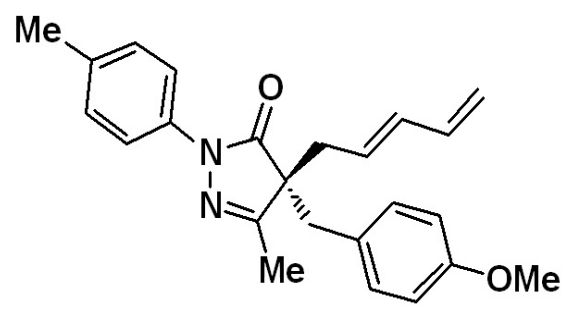

$5 d a$
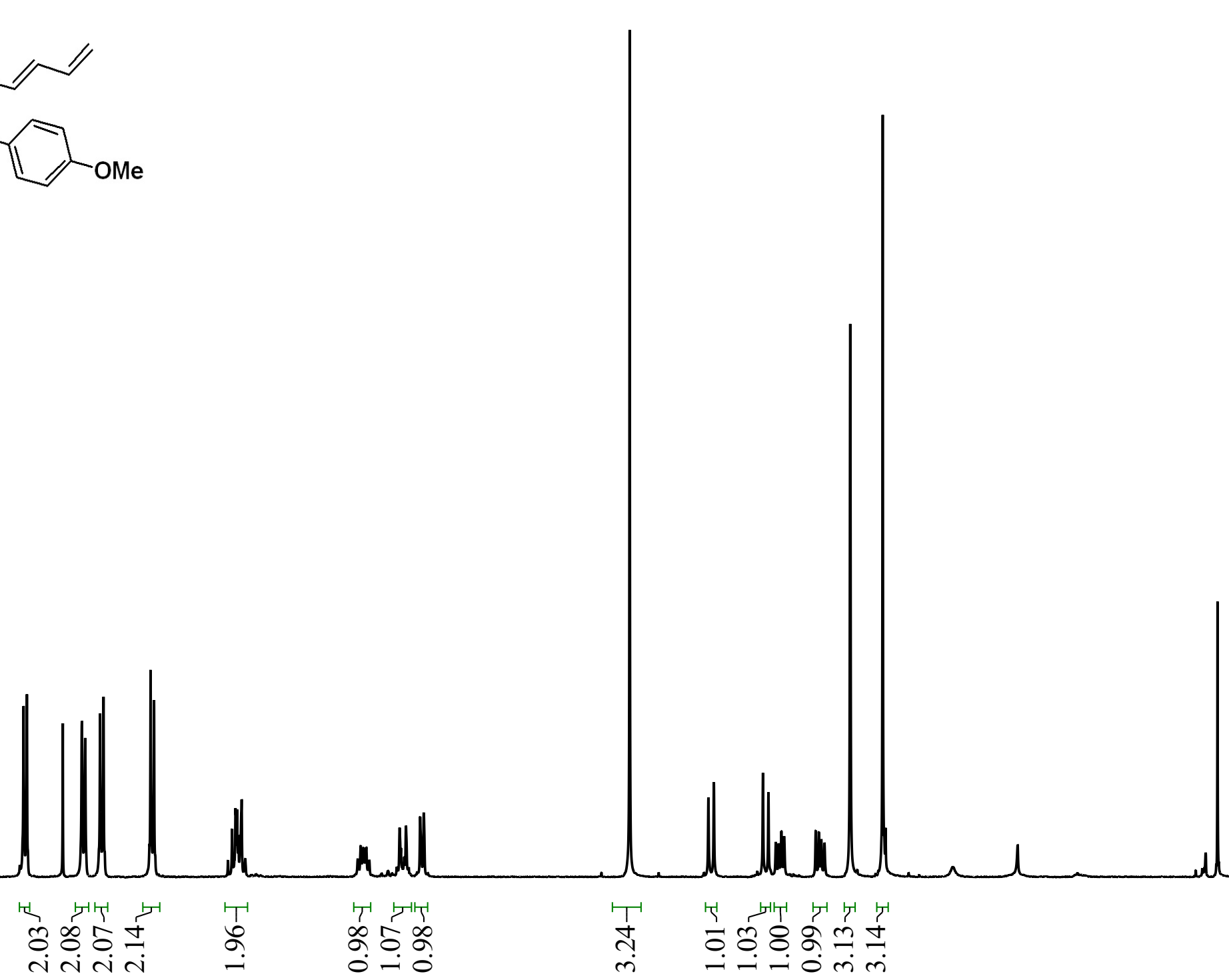

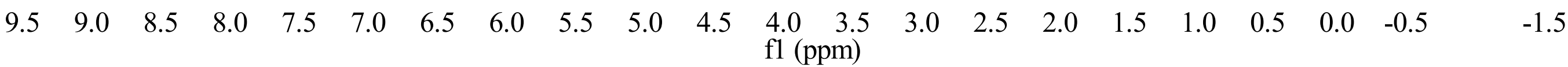




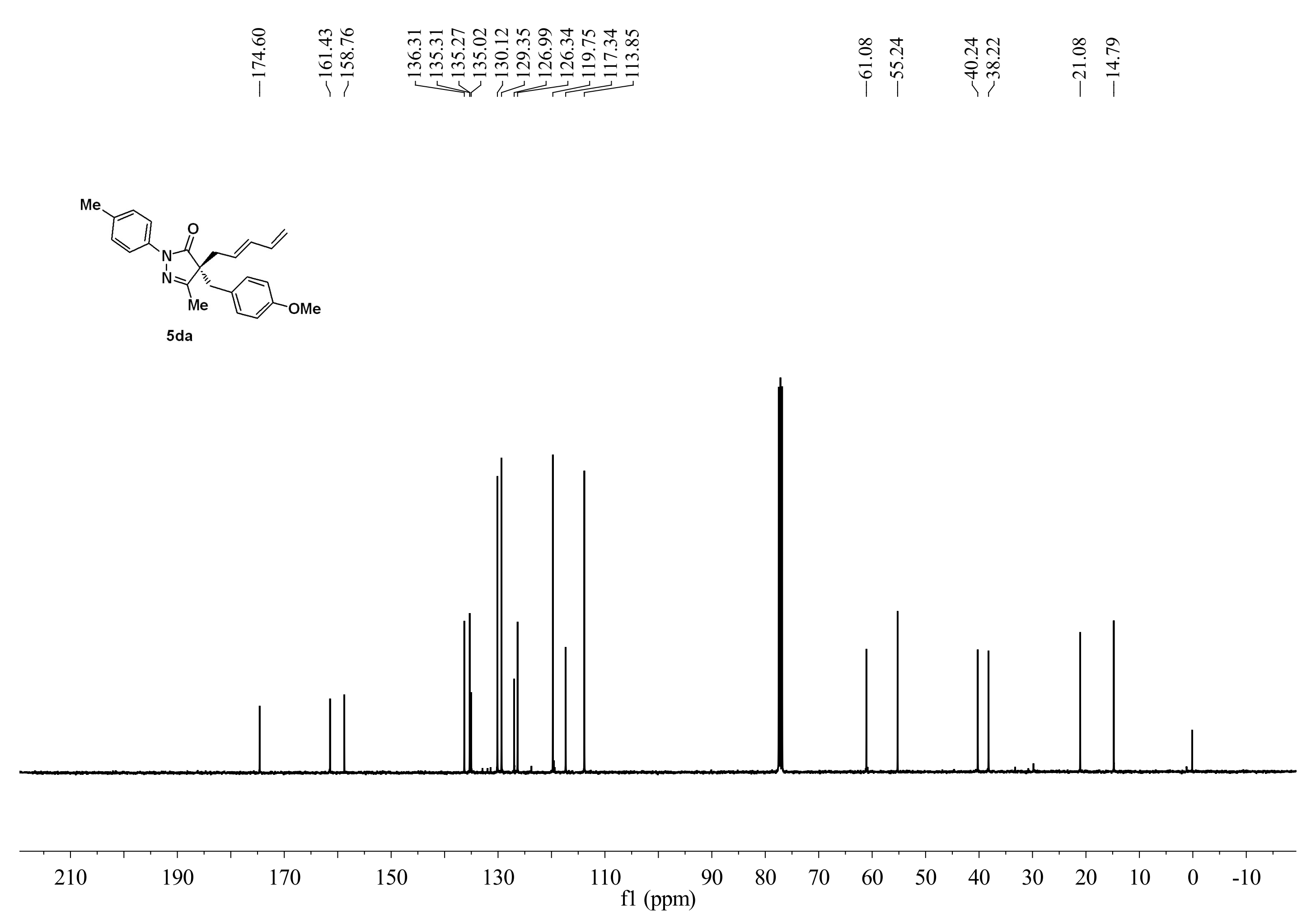


Me

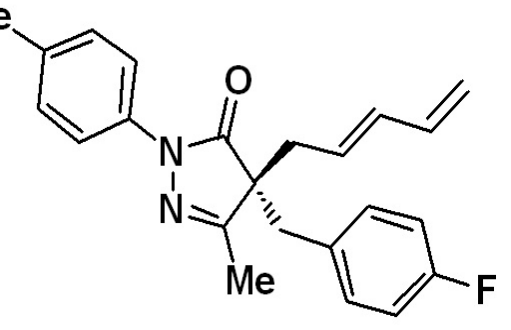

5 ea

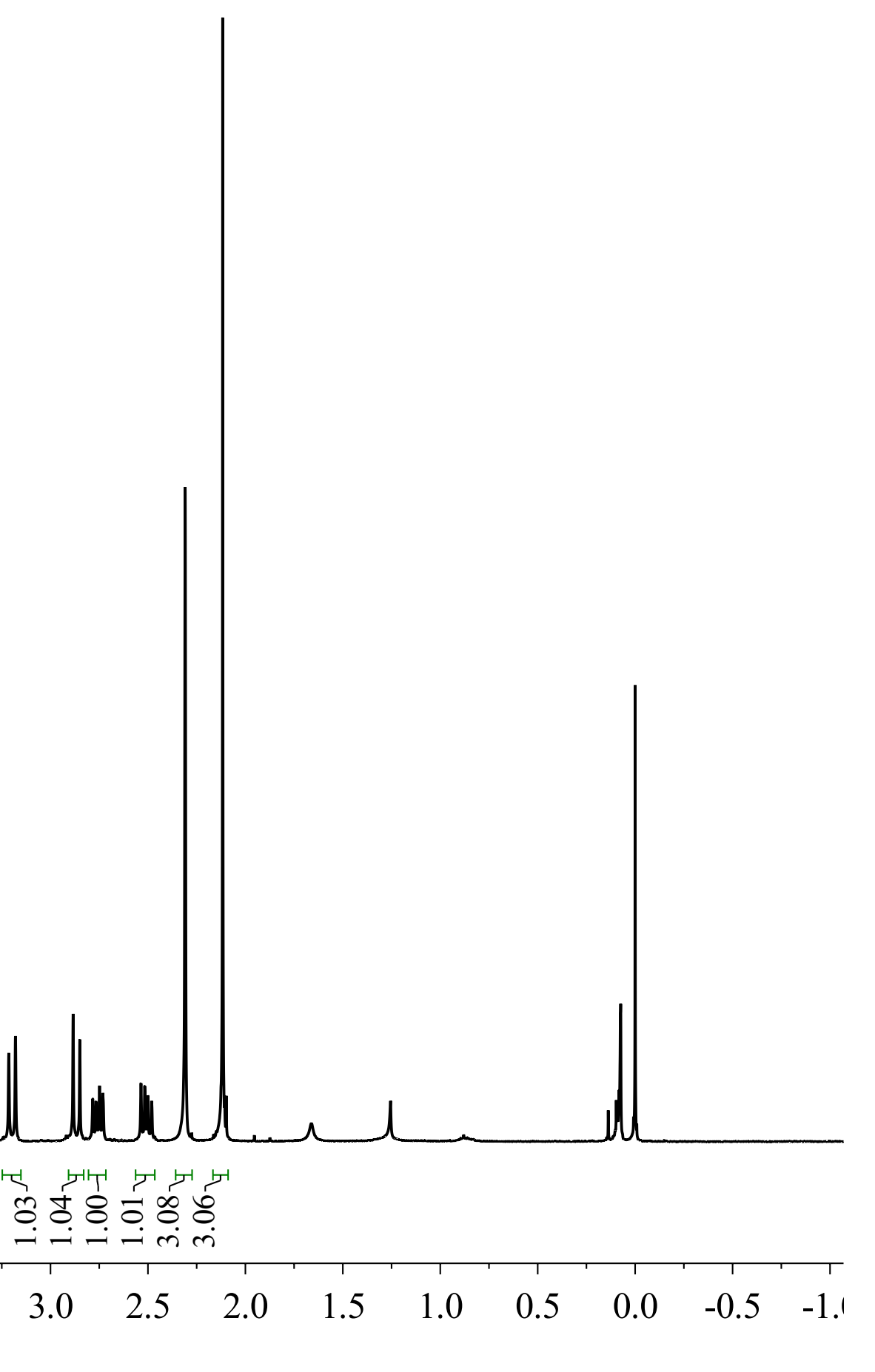




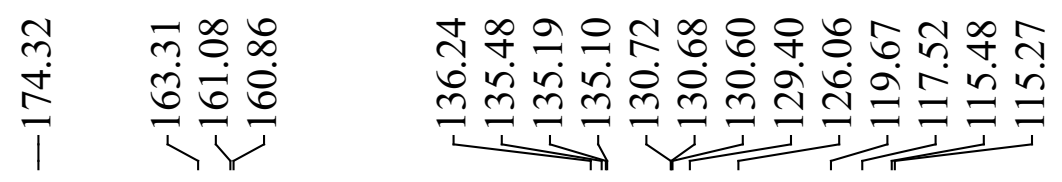

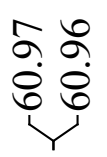

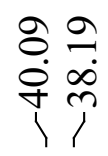

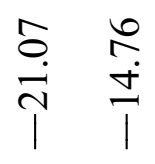<smiles>C=C/C=C/CC1(Cc2ccc(F)cc2)C(=O)N(c2ccc(C)cc2)N=C1C</smiles>

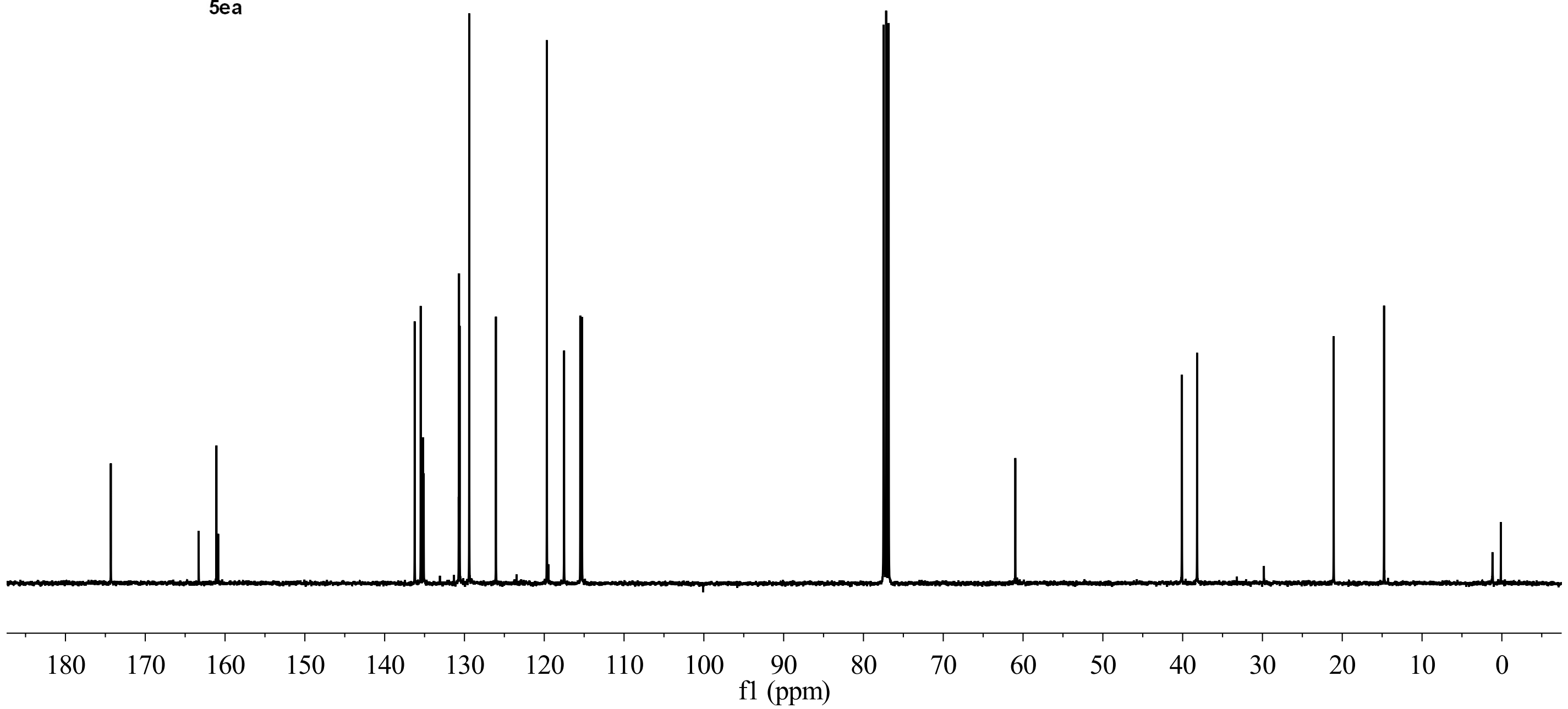




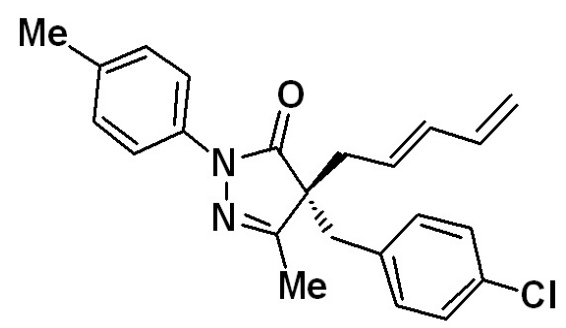

$5 f a$

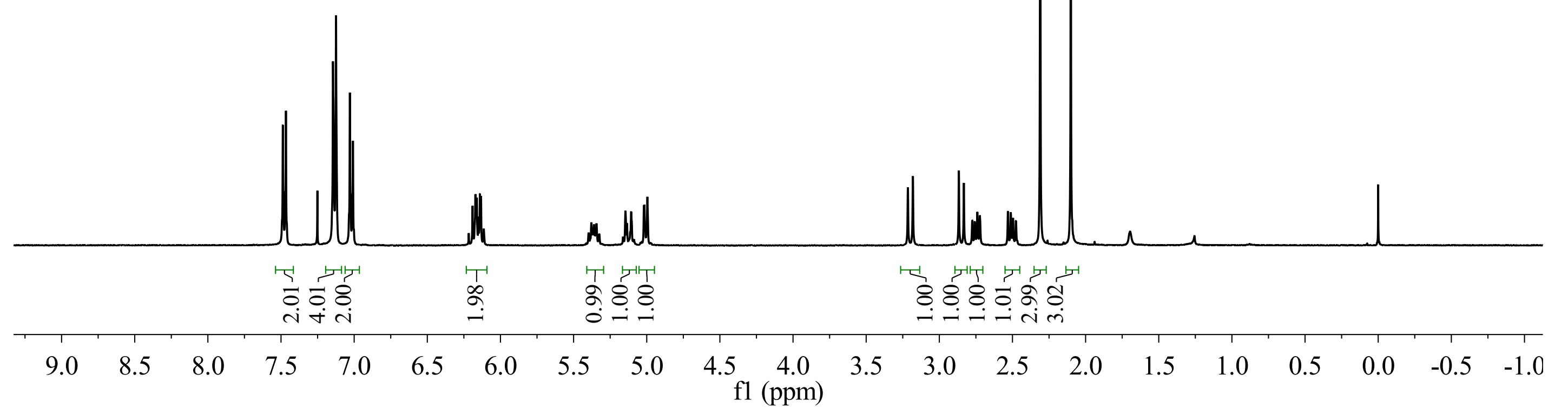


Me
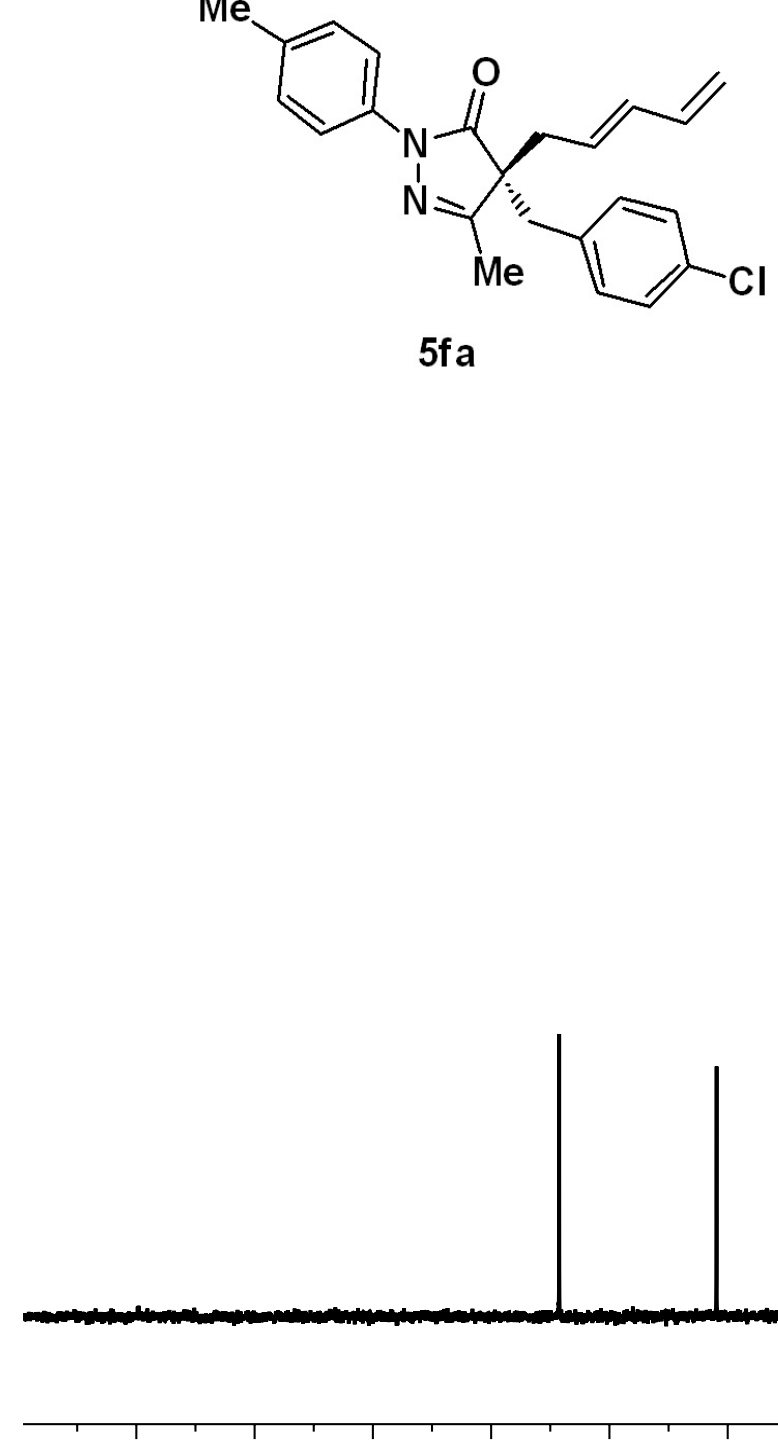

$90 \quad 80 \quad 70 \quad 60$

50

40 
负

$\mathrm{Me}$

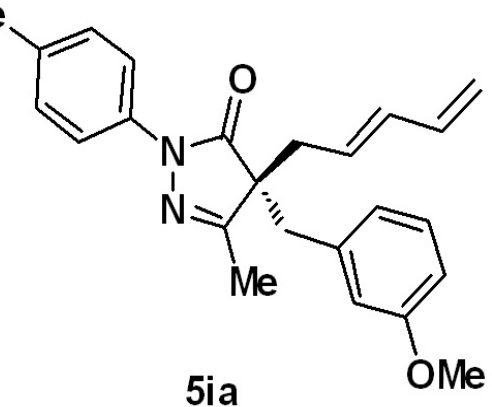$$
\text { . }
$$

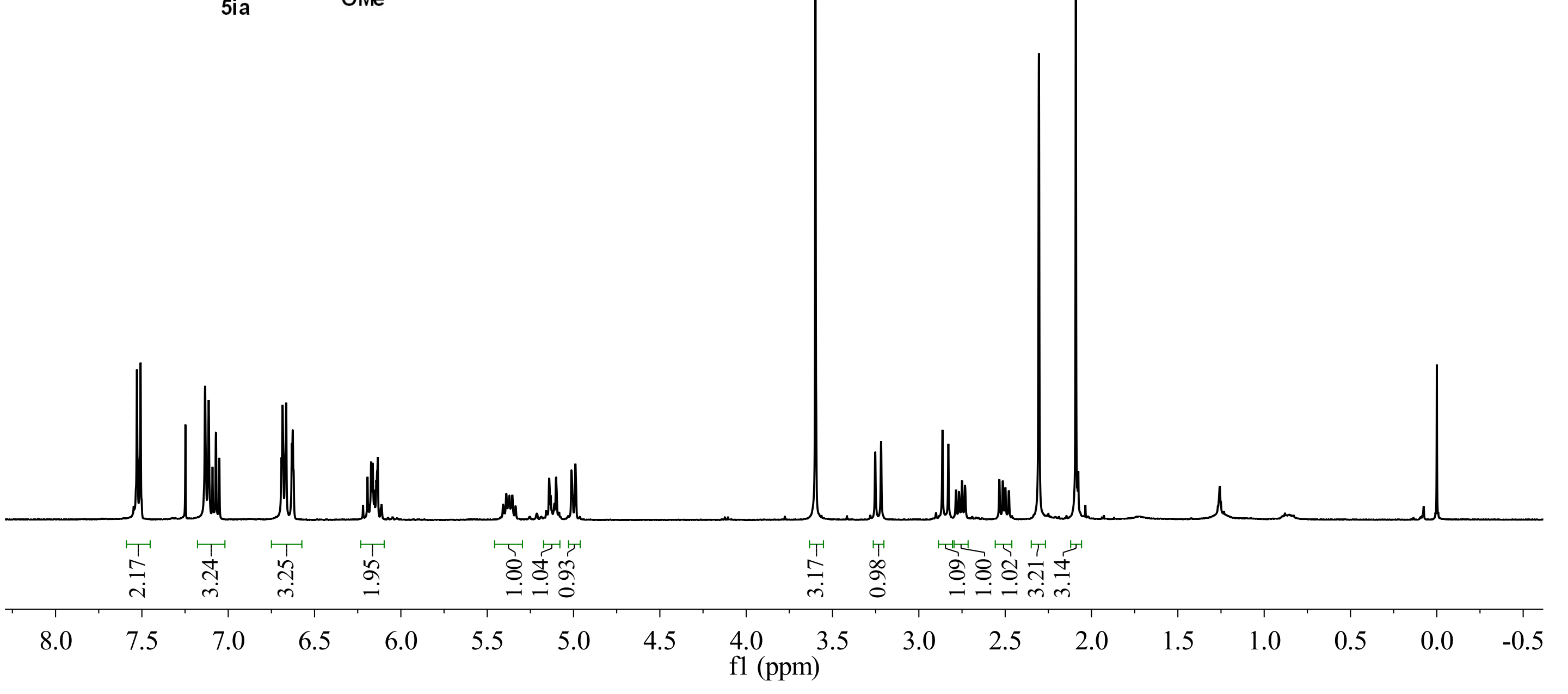



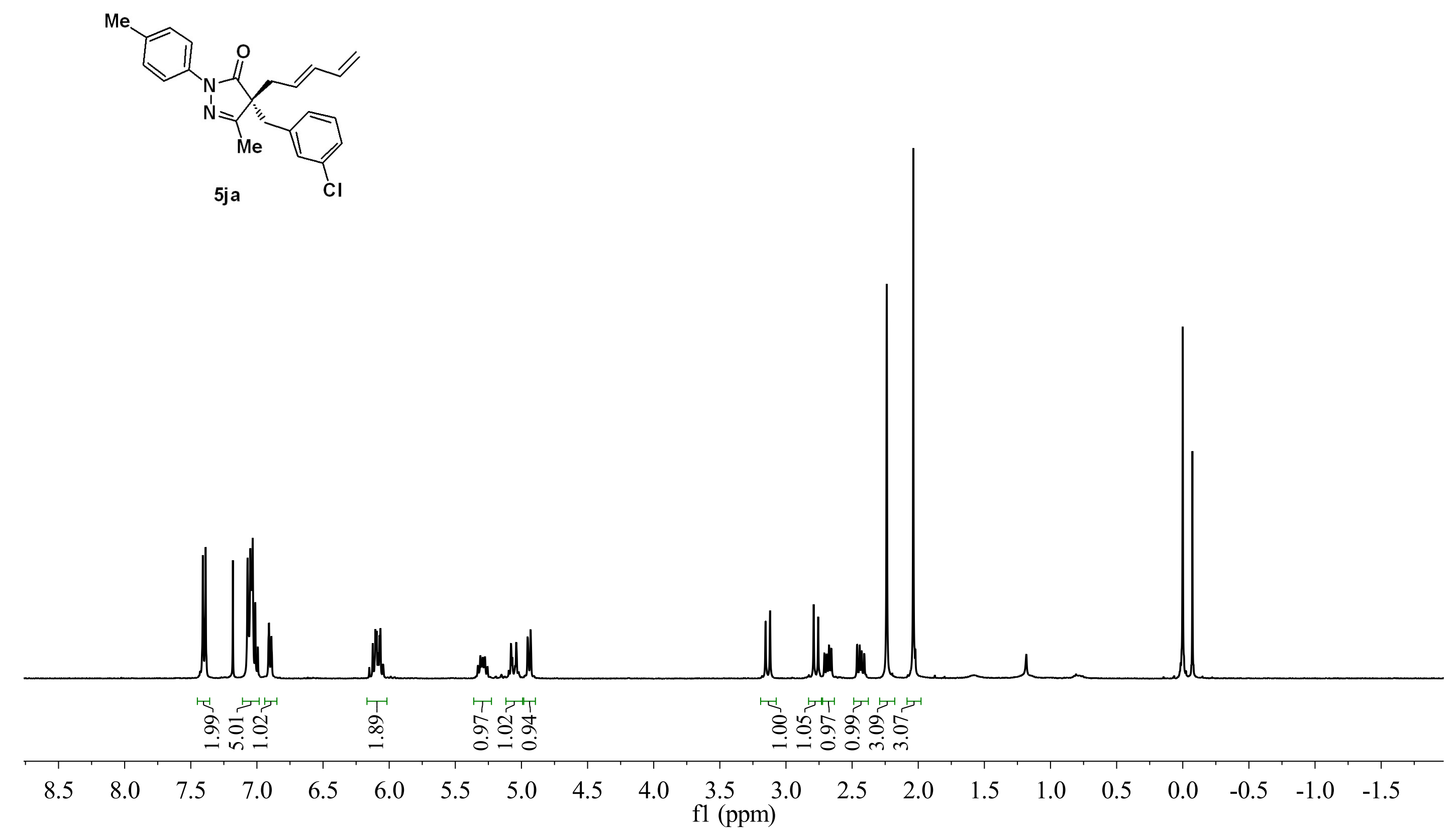


$$
\text { wl }
$$




\section{负}

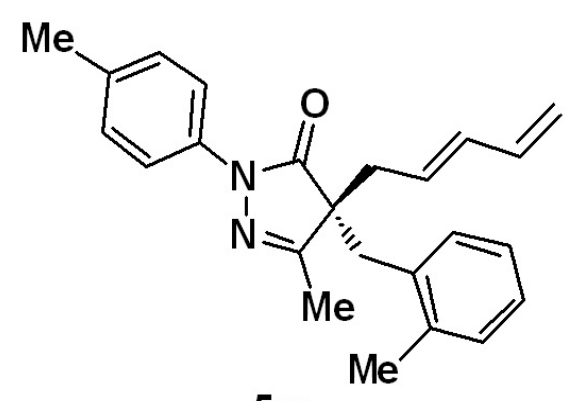

5ra

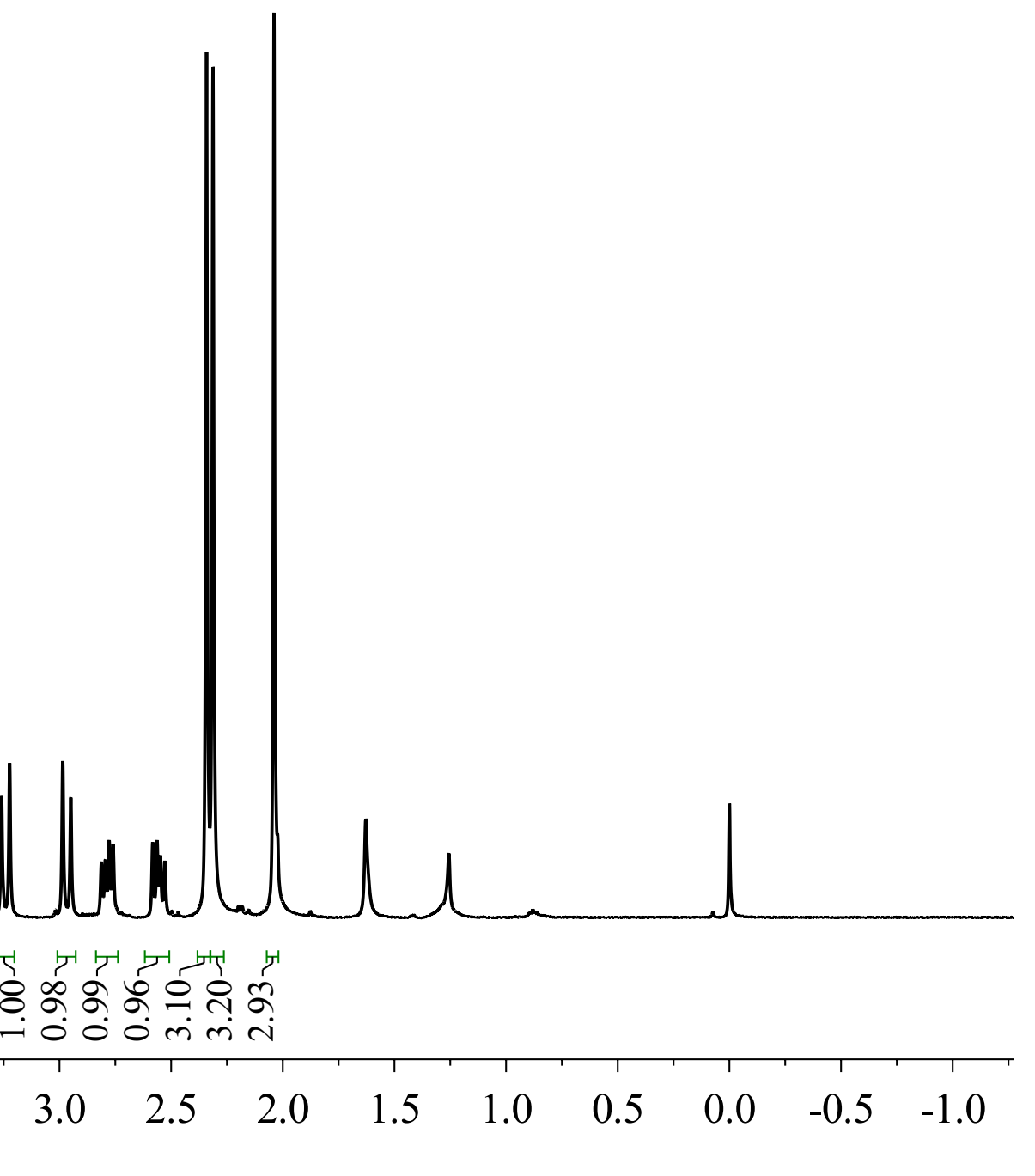


$\mathrm{Me}$

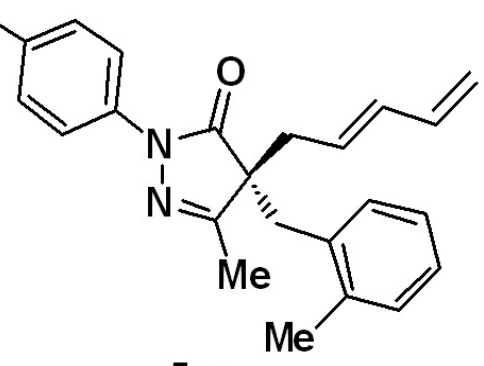

5ra

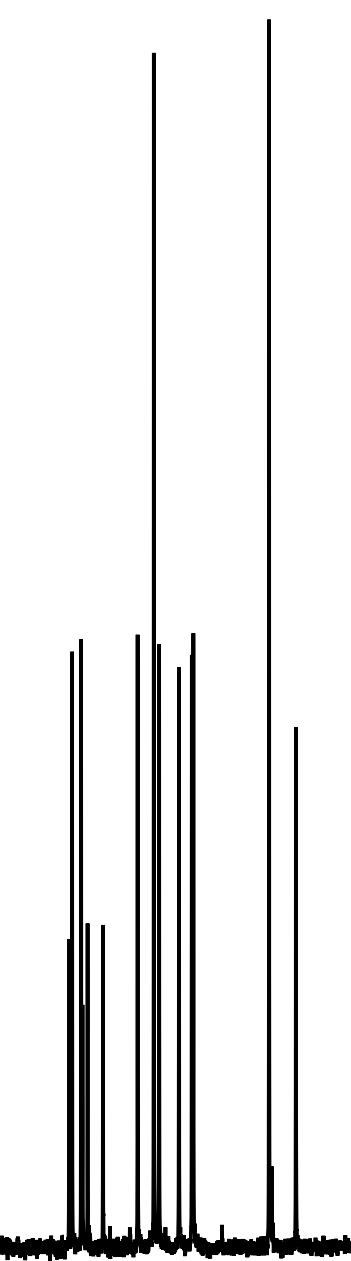

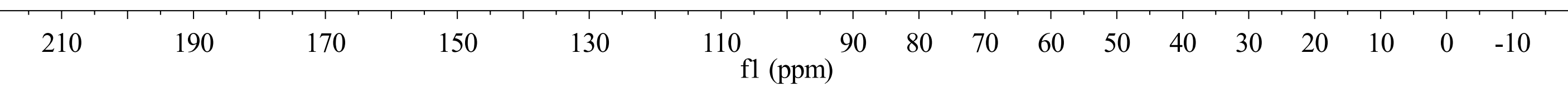




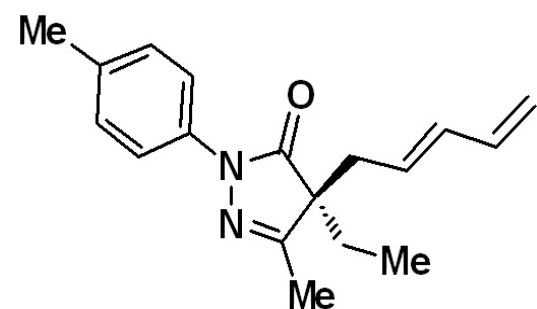

$50 a$

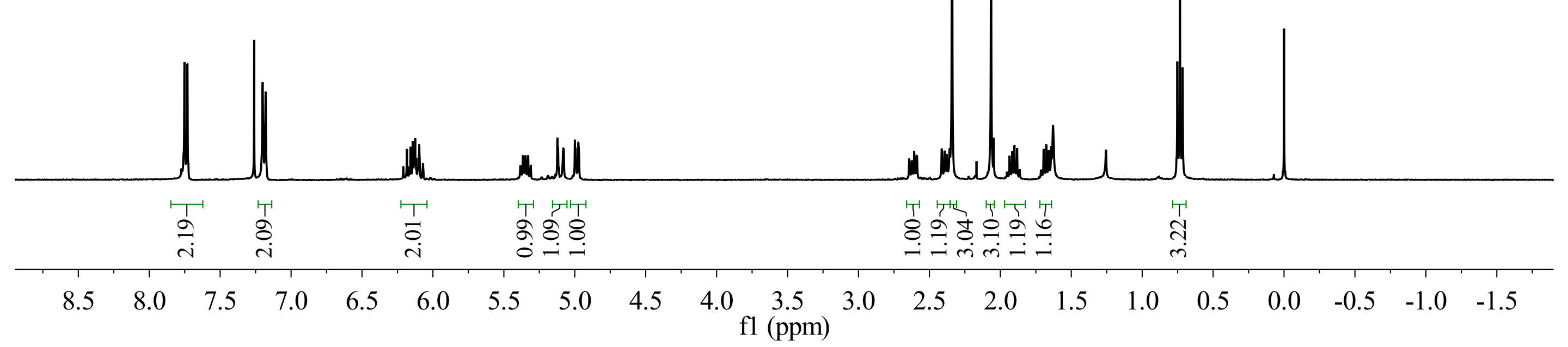




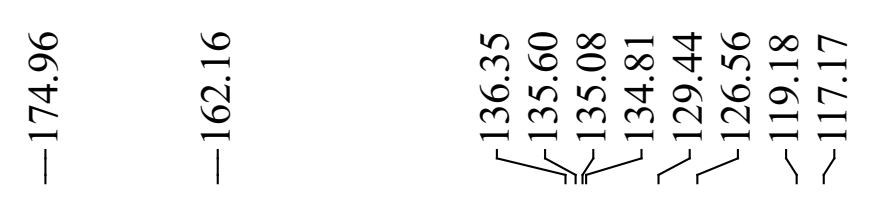

궁

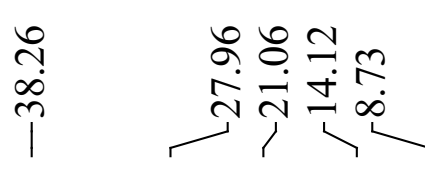

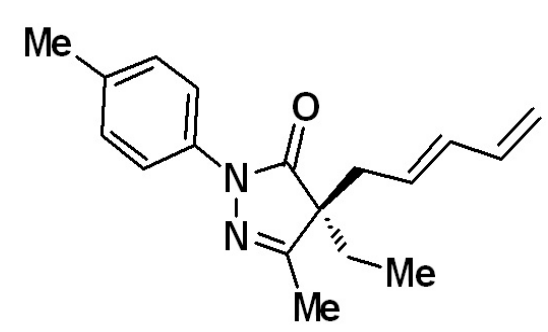

$50 a$
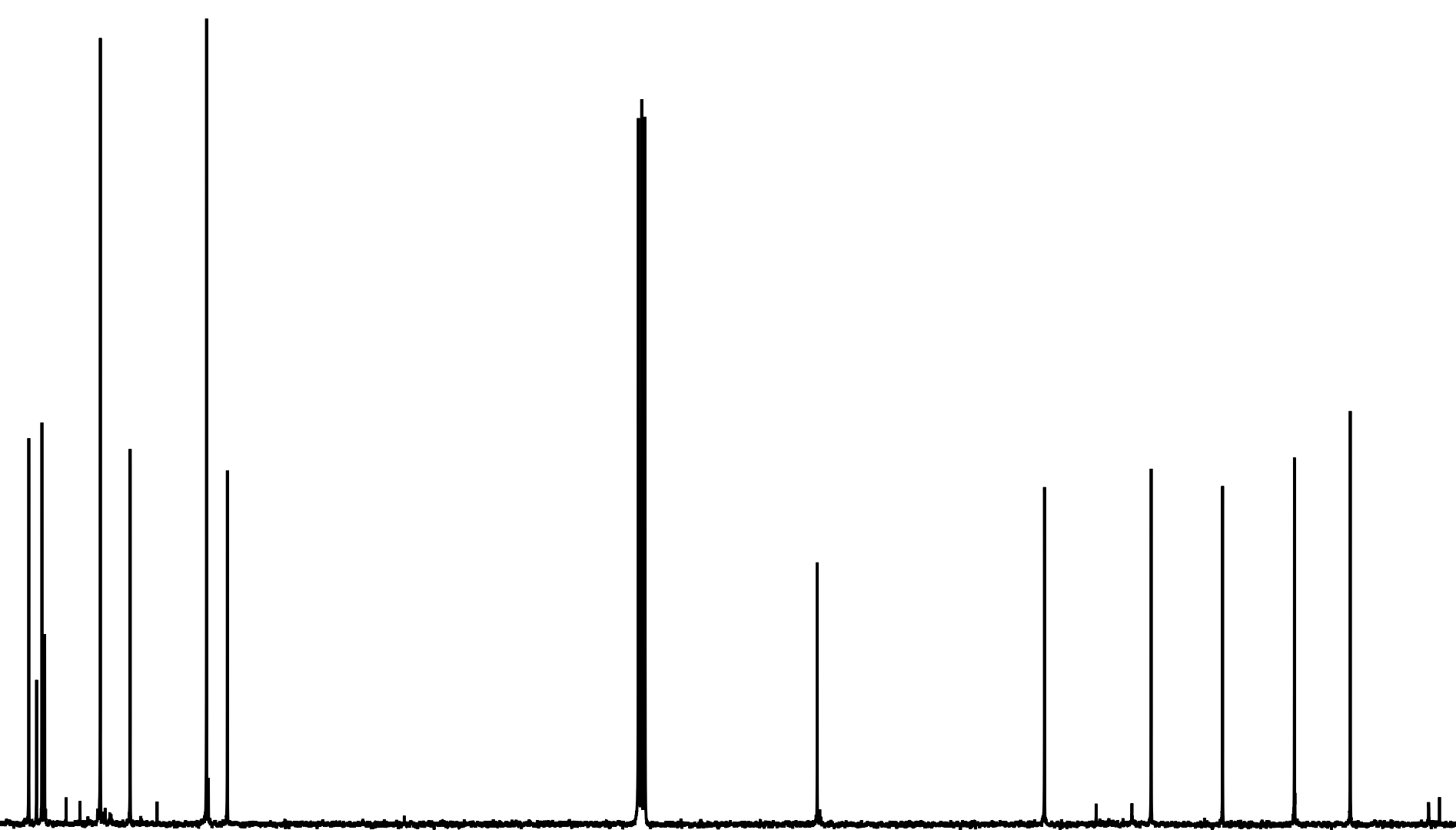

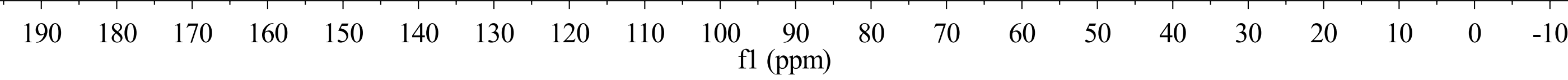




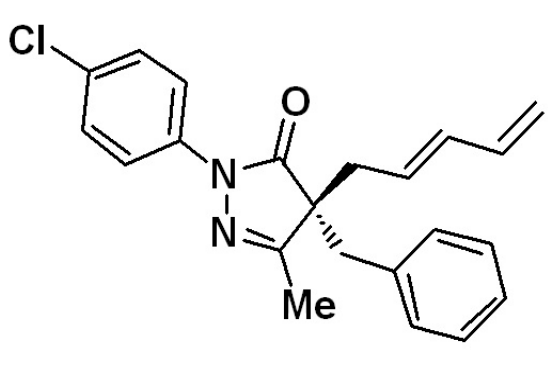

$5 \mathrm{pa}$

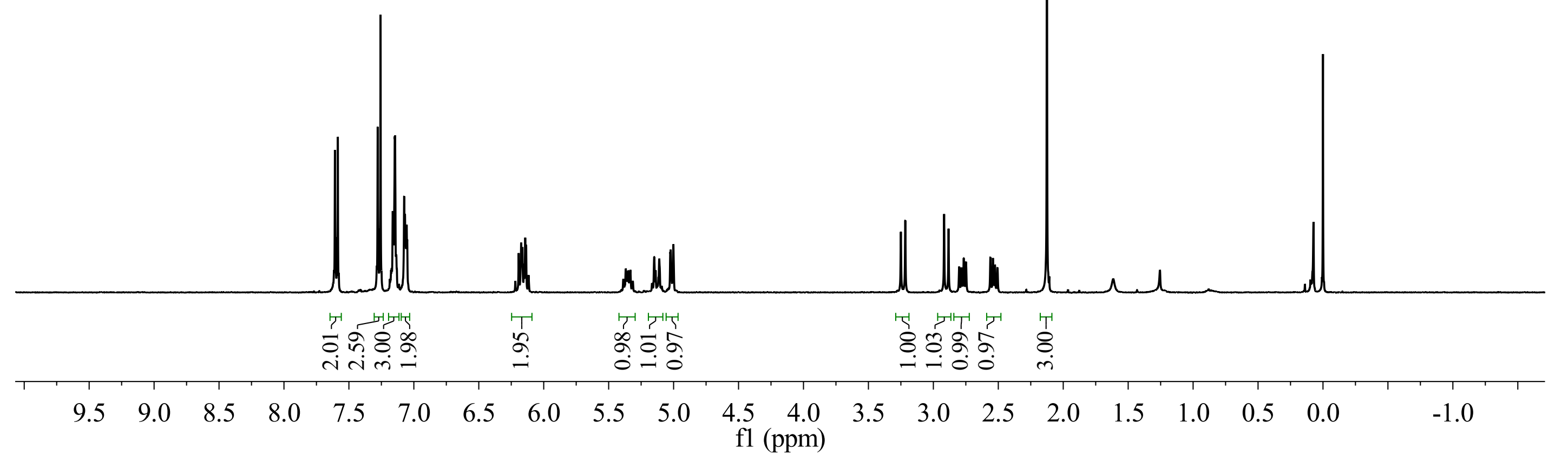




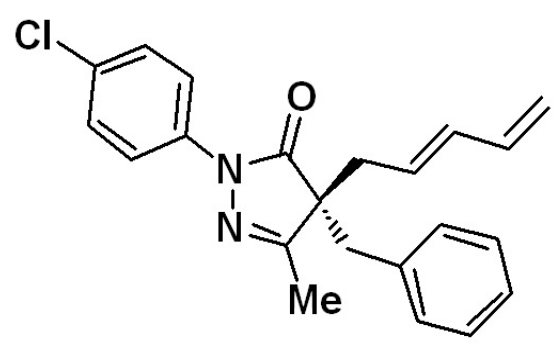

$5 p a$

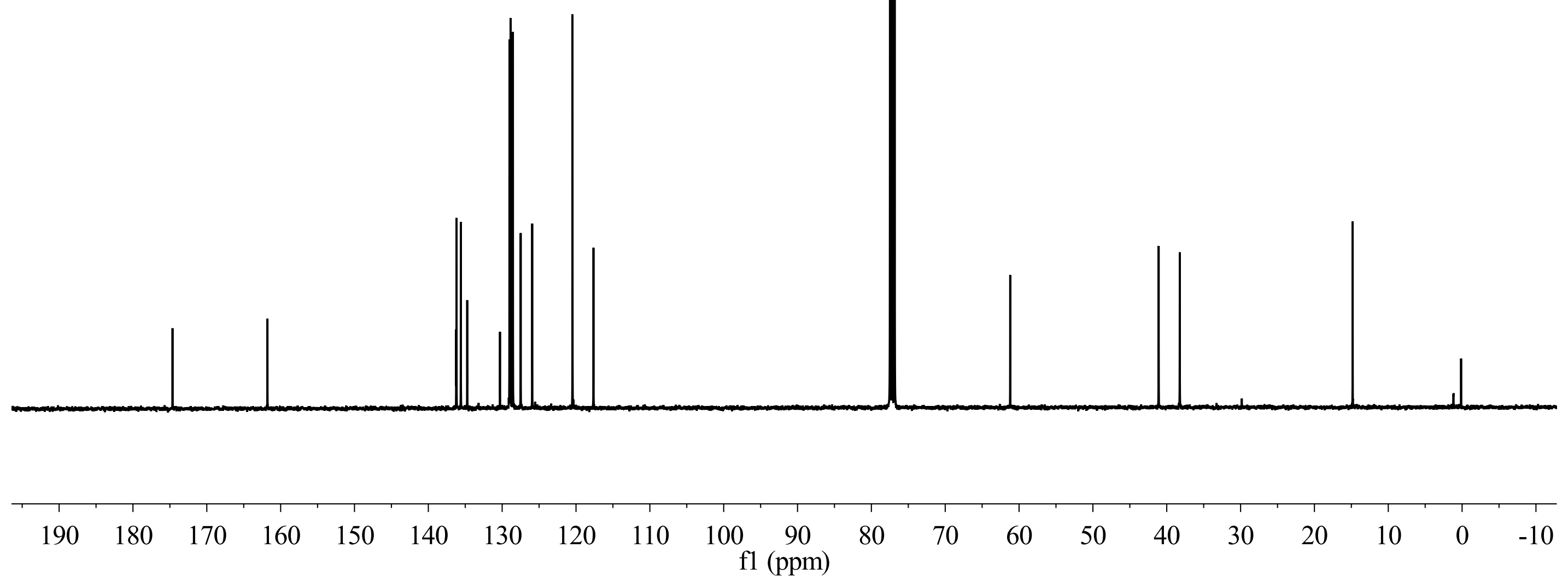




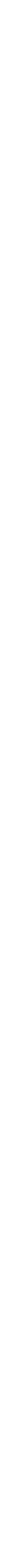




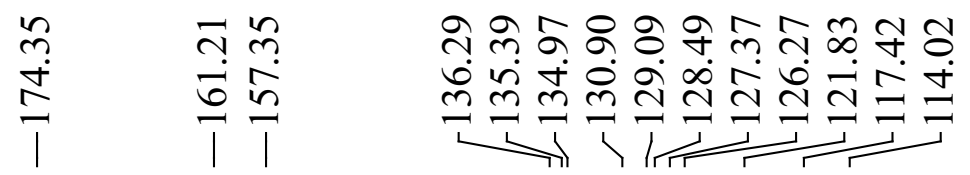

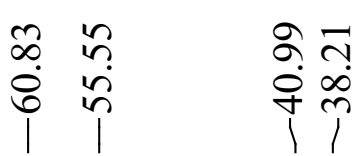

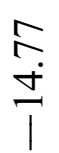

$\mathrm{MeO}$

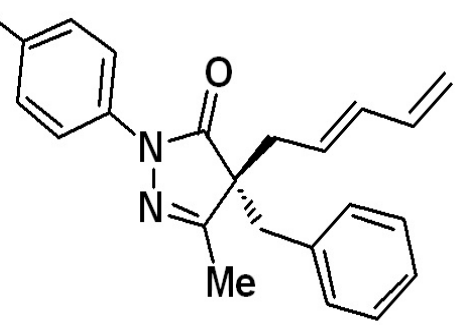

5sa
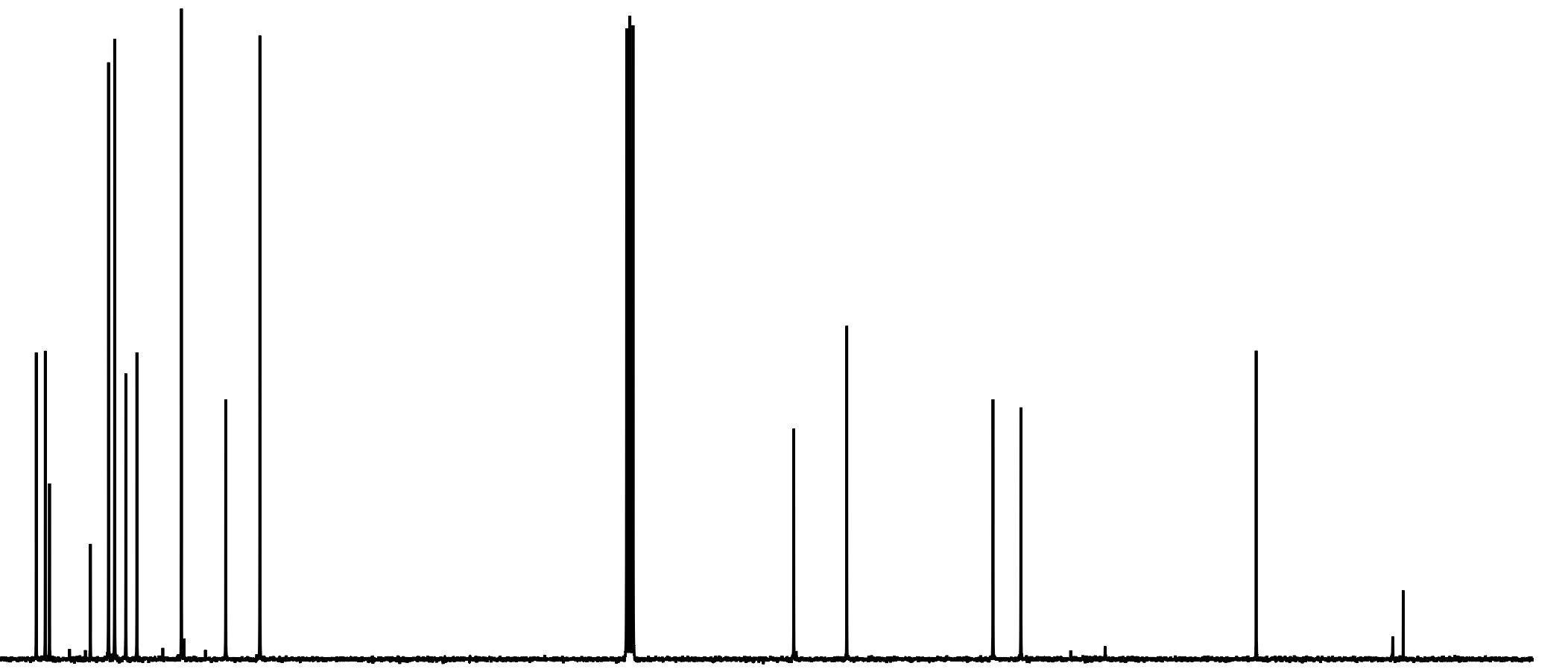

$\begin{array}{lllllllllllllllllllll}190 & 180 & 170 & 160 & 150 & 140 & 130 & 120 & 110 & 100 & 90 & 80 & 70 & 60 & 50 & 40 & 30 & 20 & 10 & 0 & -10\end{array}$ 


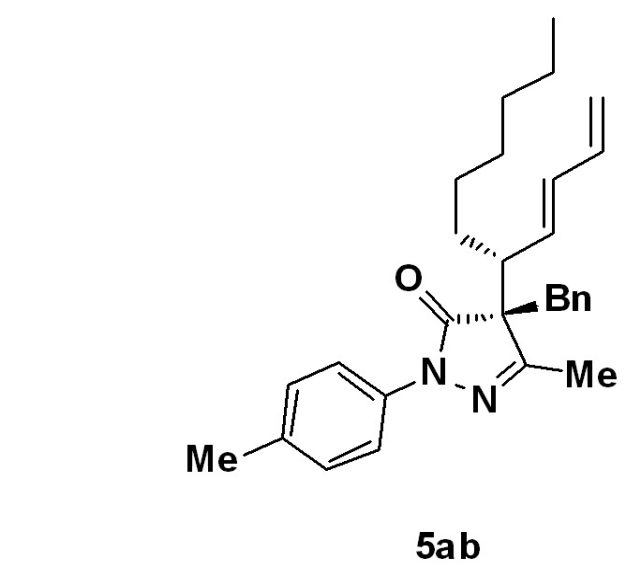

$5 a b$

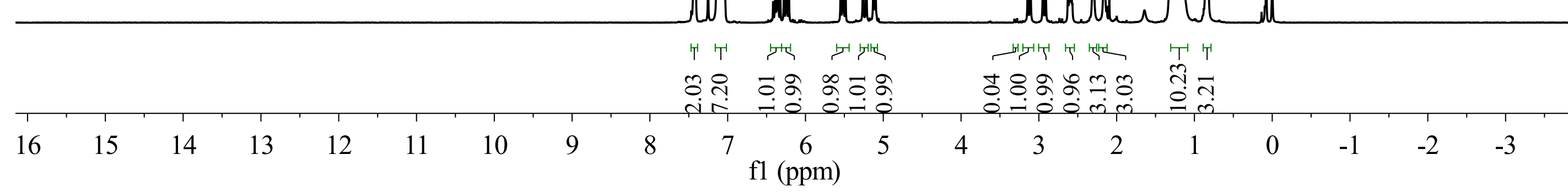




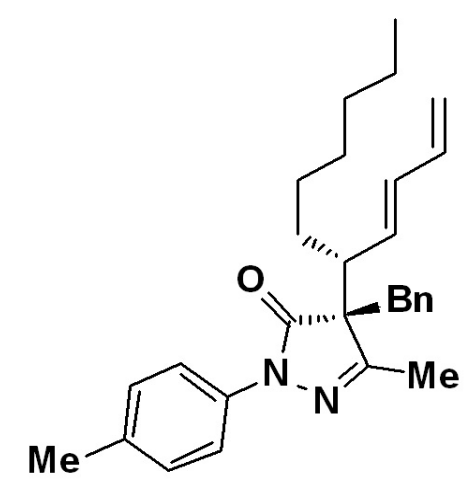

$5 \mathbf{a b}$

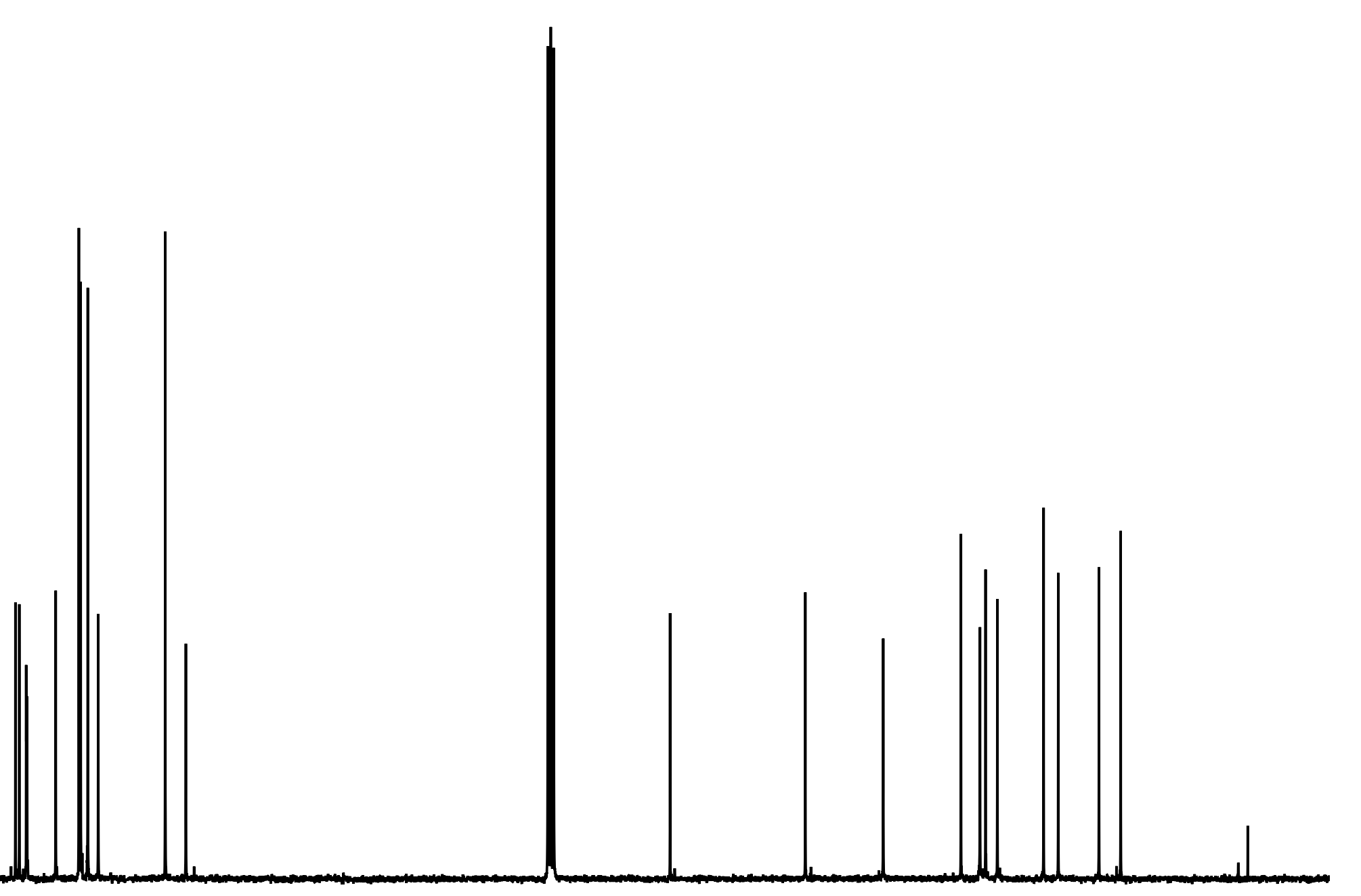




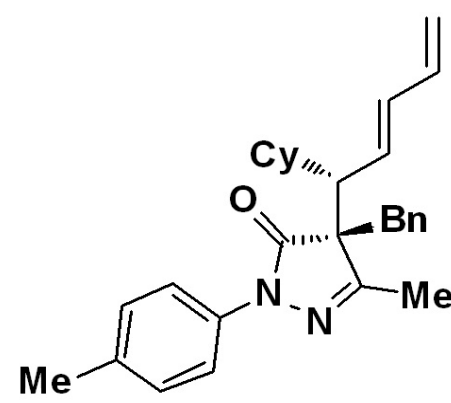

5 ac

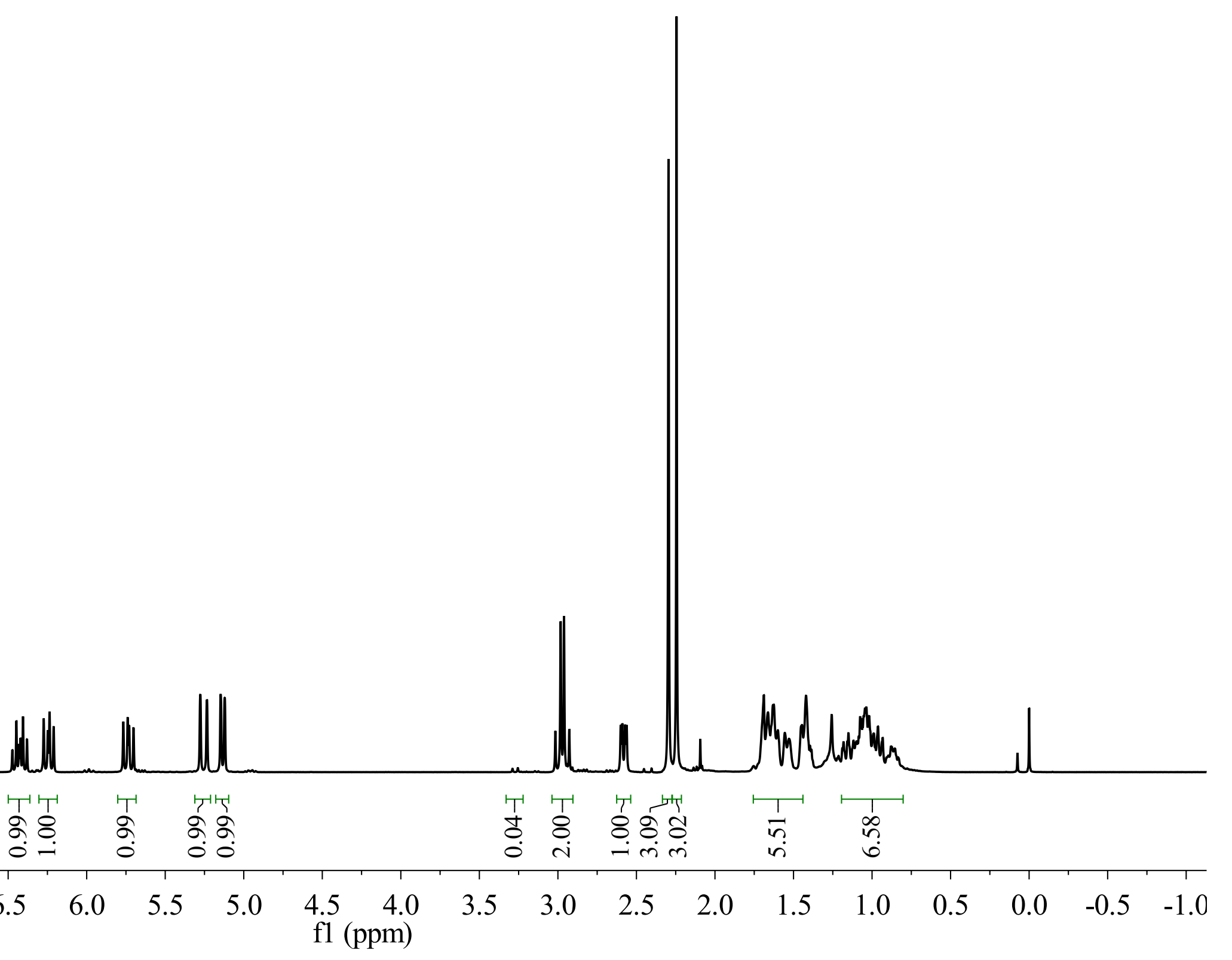




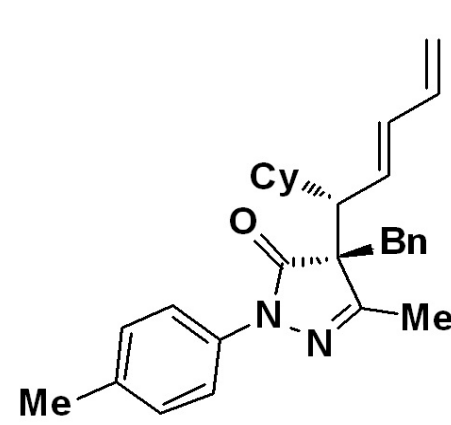

$5 a c$

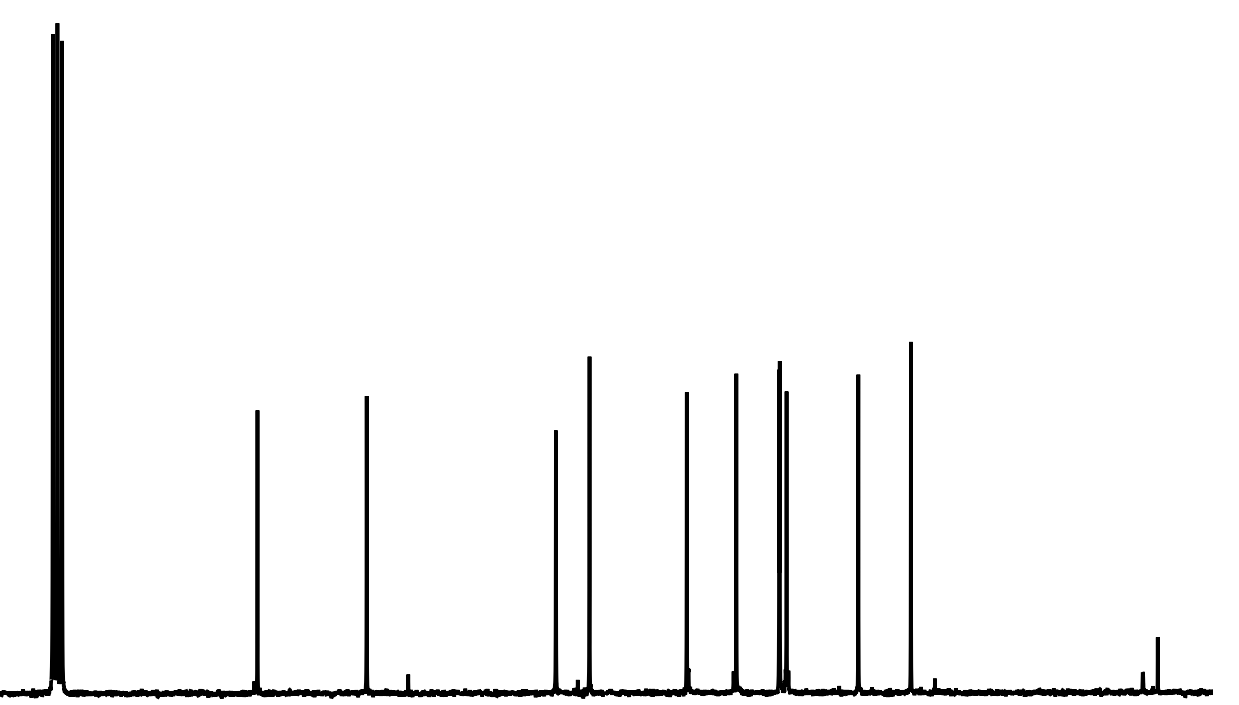

\begin{tabular}{|c|c|c|c|c|c|c|c|c|c|c|c|c|c|c|c|c|c|}
\hline & 180 & 170 & 160 & 150 & 140 & 130 & 120 & 110 & \begin{tabular}{c|c} 
& \\
100 & 90
\end{tabular} & 80 & 70 & 60 & 50 & 40 & 30 & 20 & 10 \\
\hline
\end{tabular}




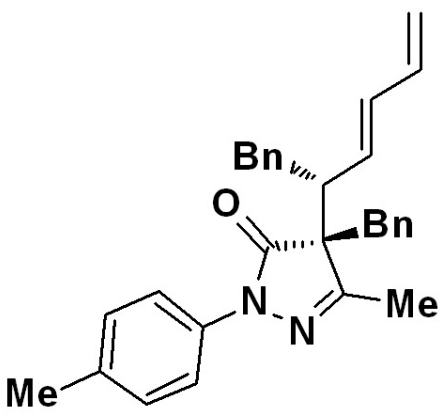

5ad

.
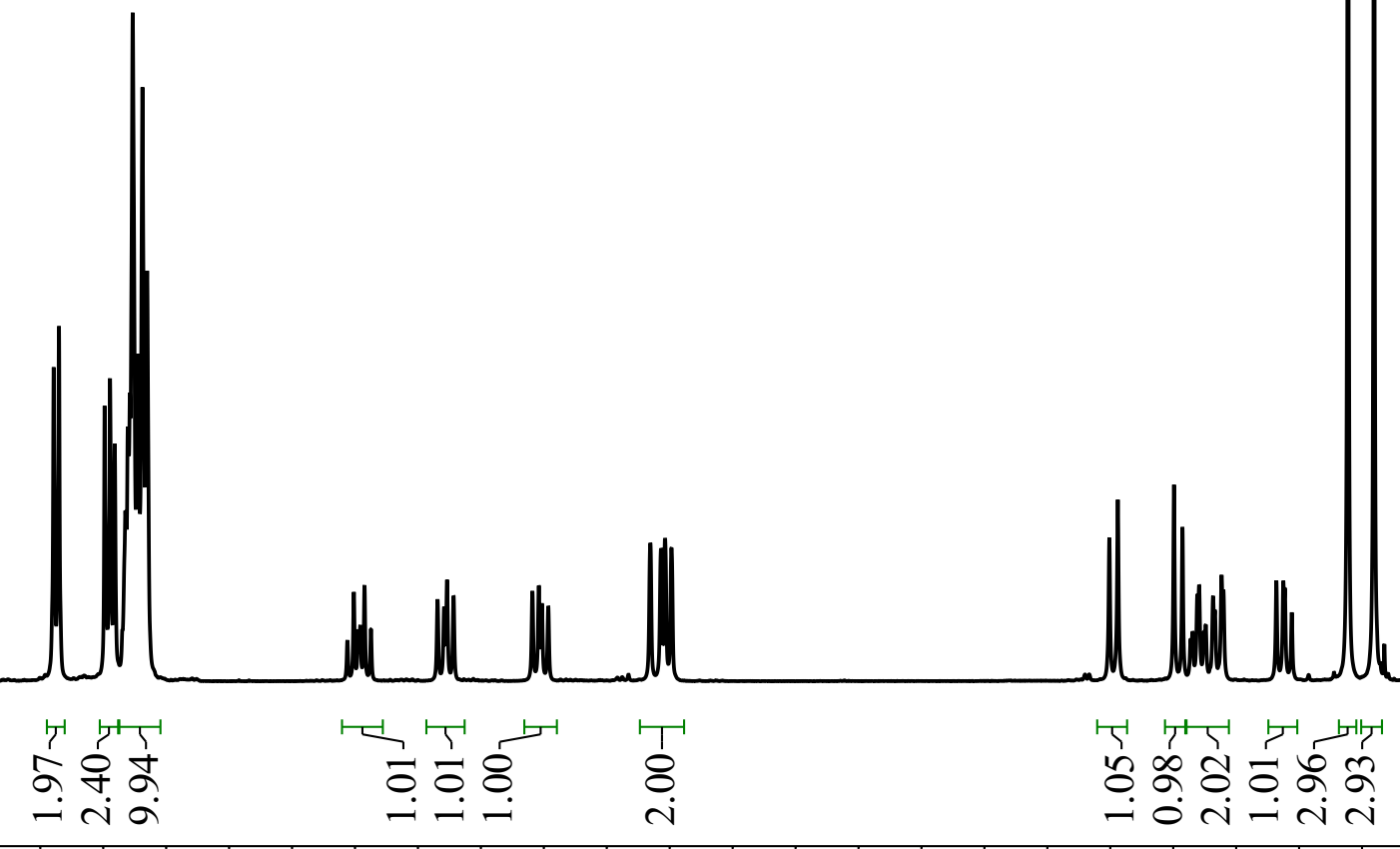

$\begin{array}{llllllllllll}9.0 & 8.5 & 8.0 & 7.5 & 7.0 & 6.5 & 6.0 & 5.5 & 5.0 & 4.5 & 4.0 & 3.5\end{array}$

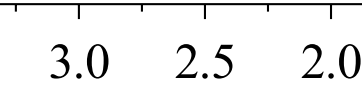




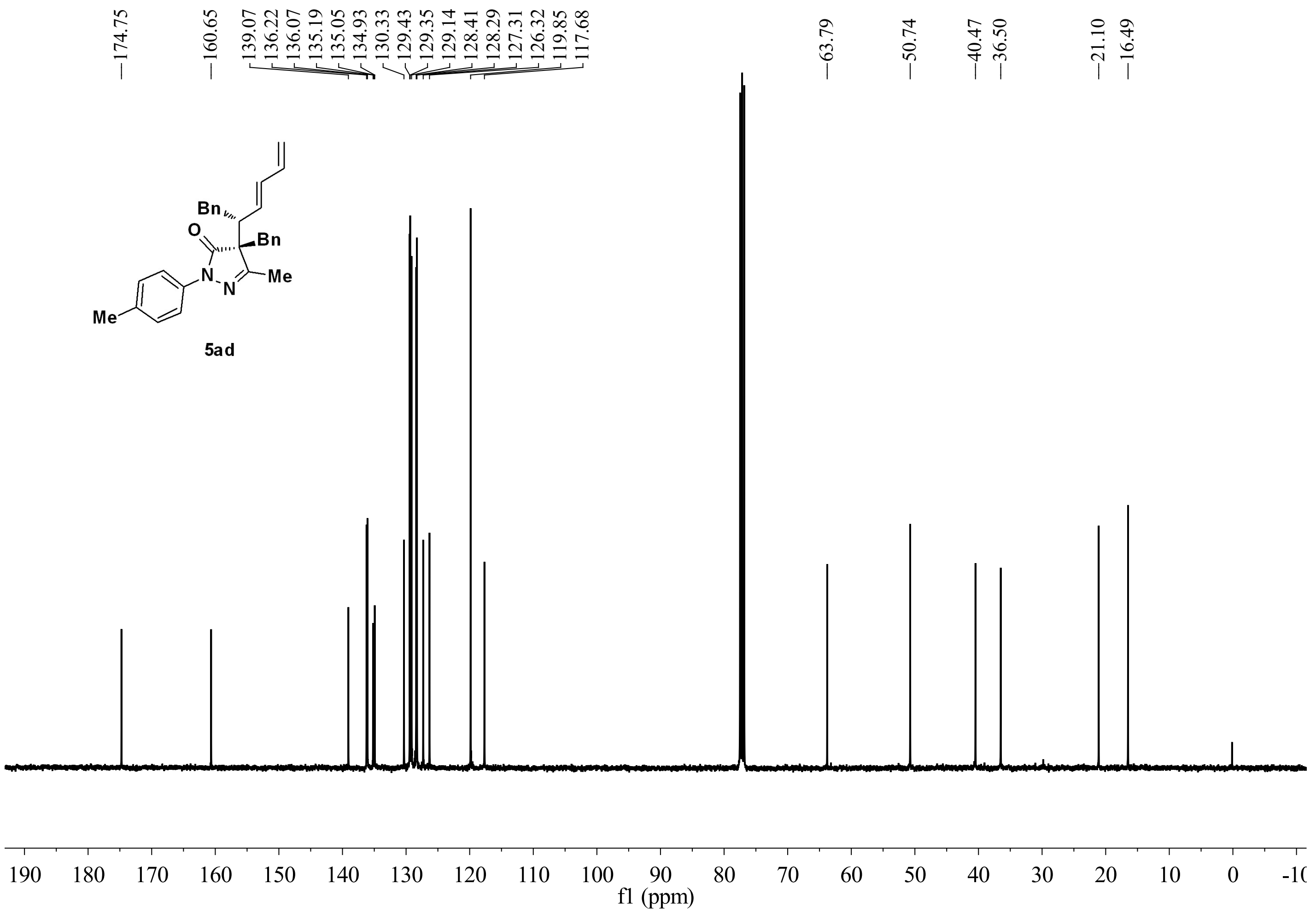



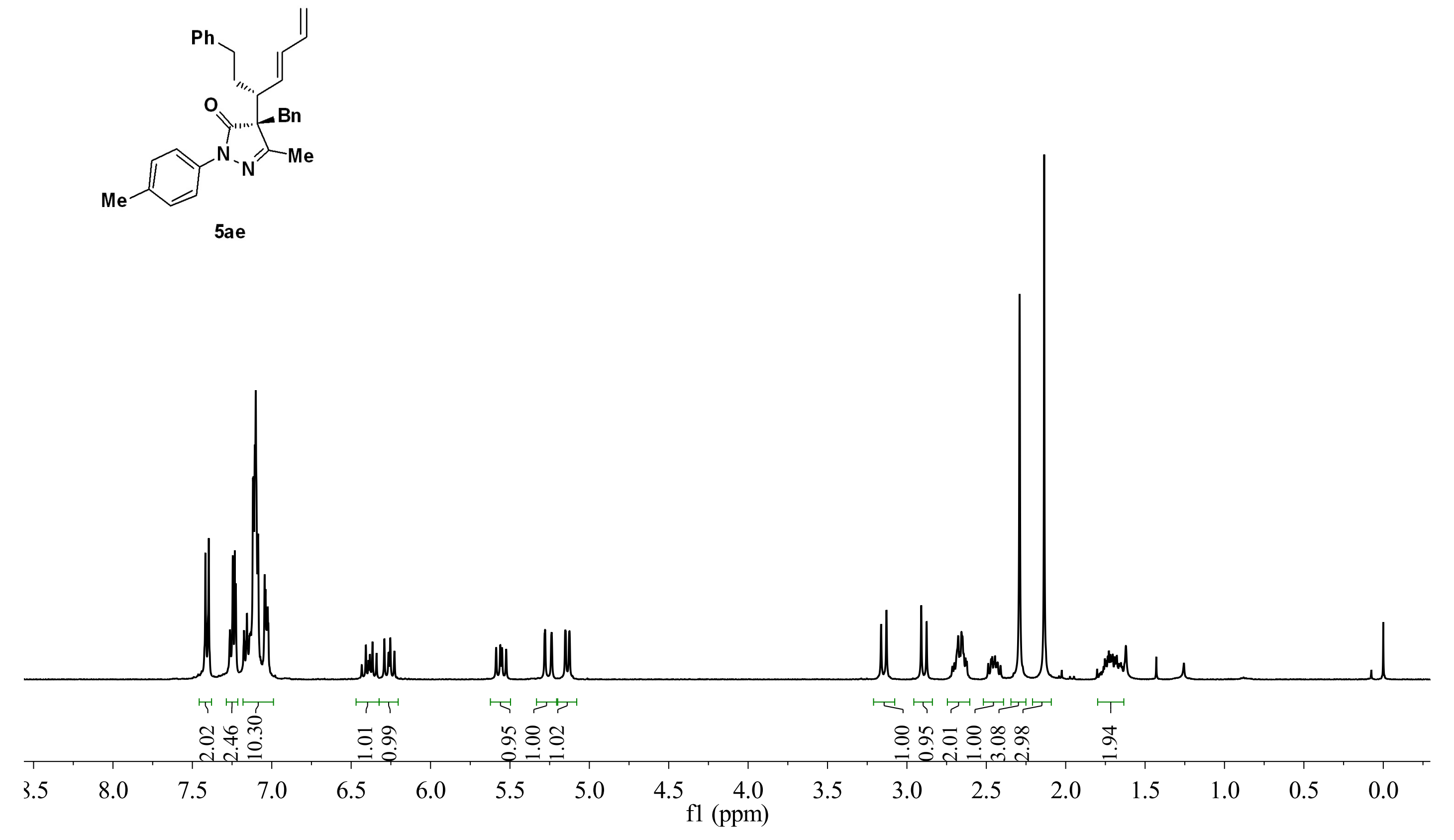


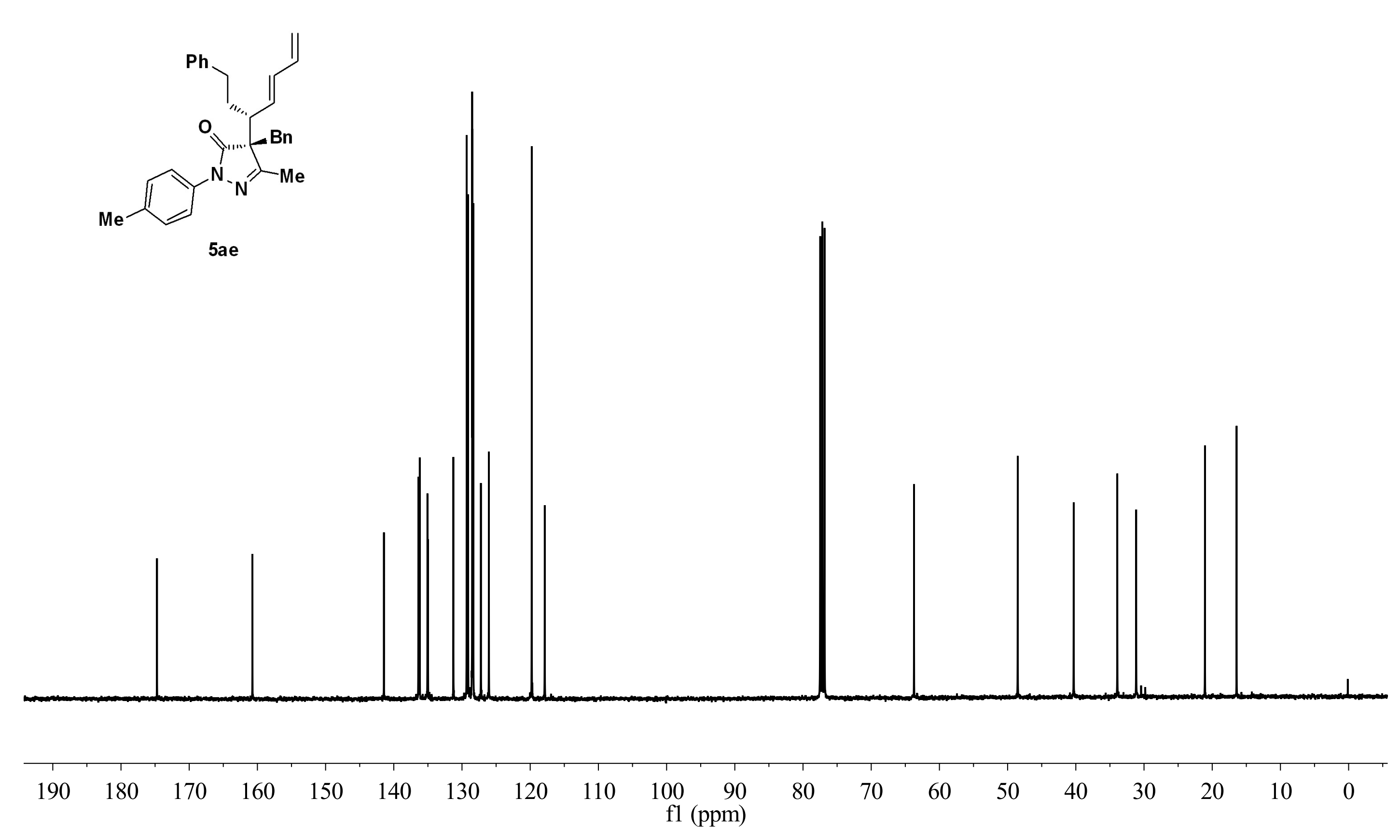



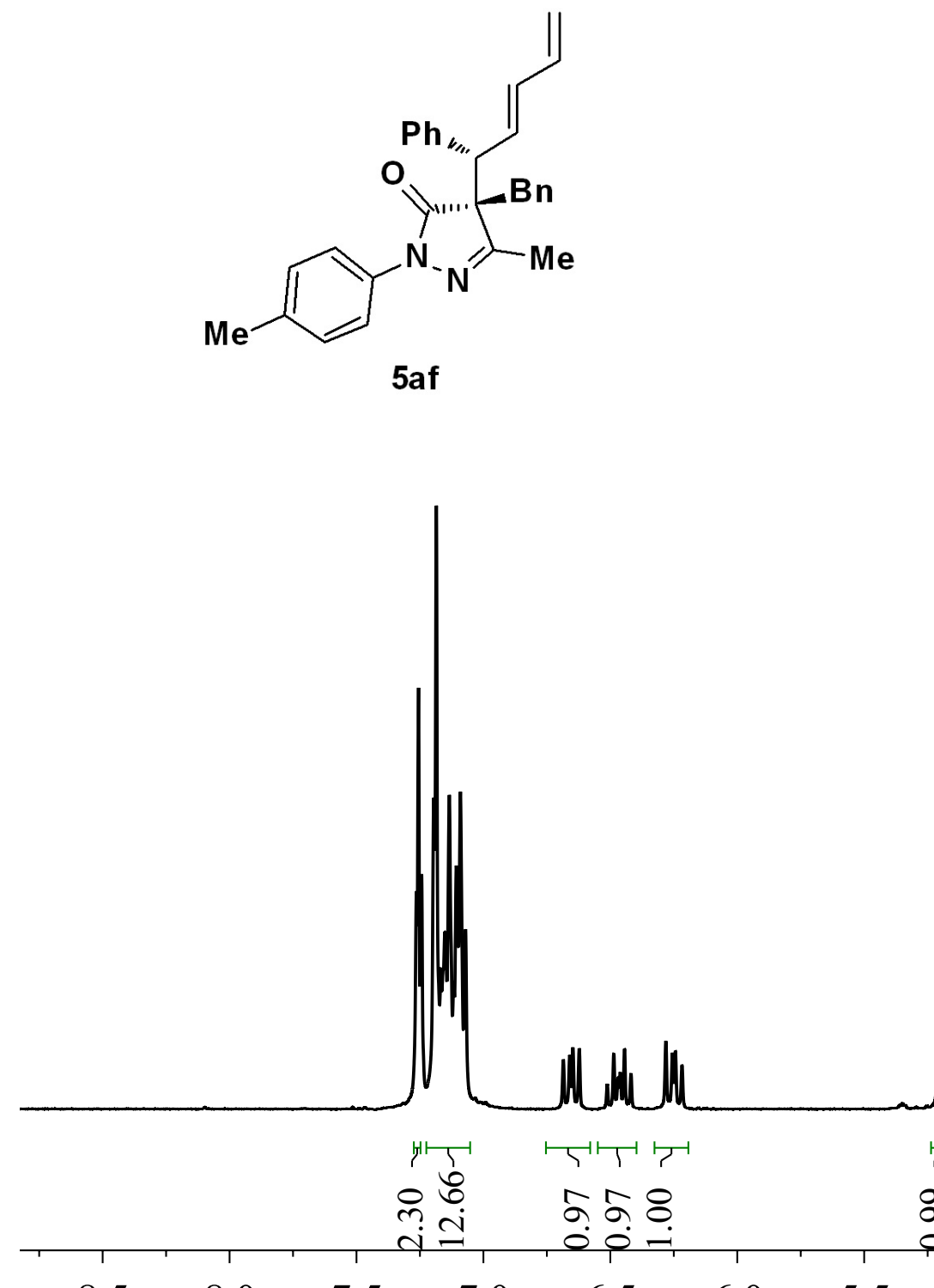

$\begin{array}{lllll}8.5 & 8.0 & 7.5 & 7.0 & 6.5\end{array}$

ริ8

5.0

$4.5 \quad 4.0 \quad 3.5$
fl (ppm)

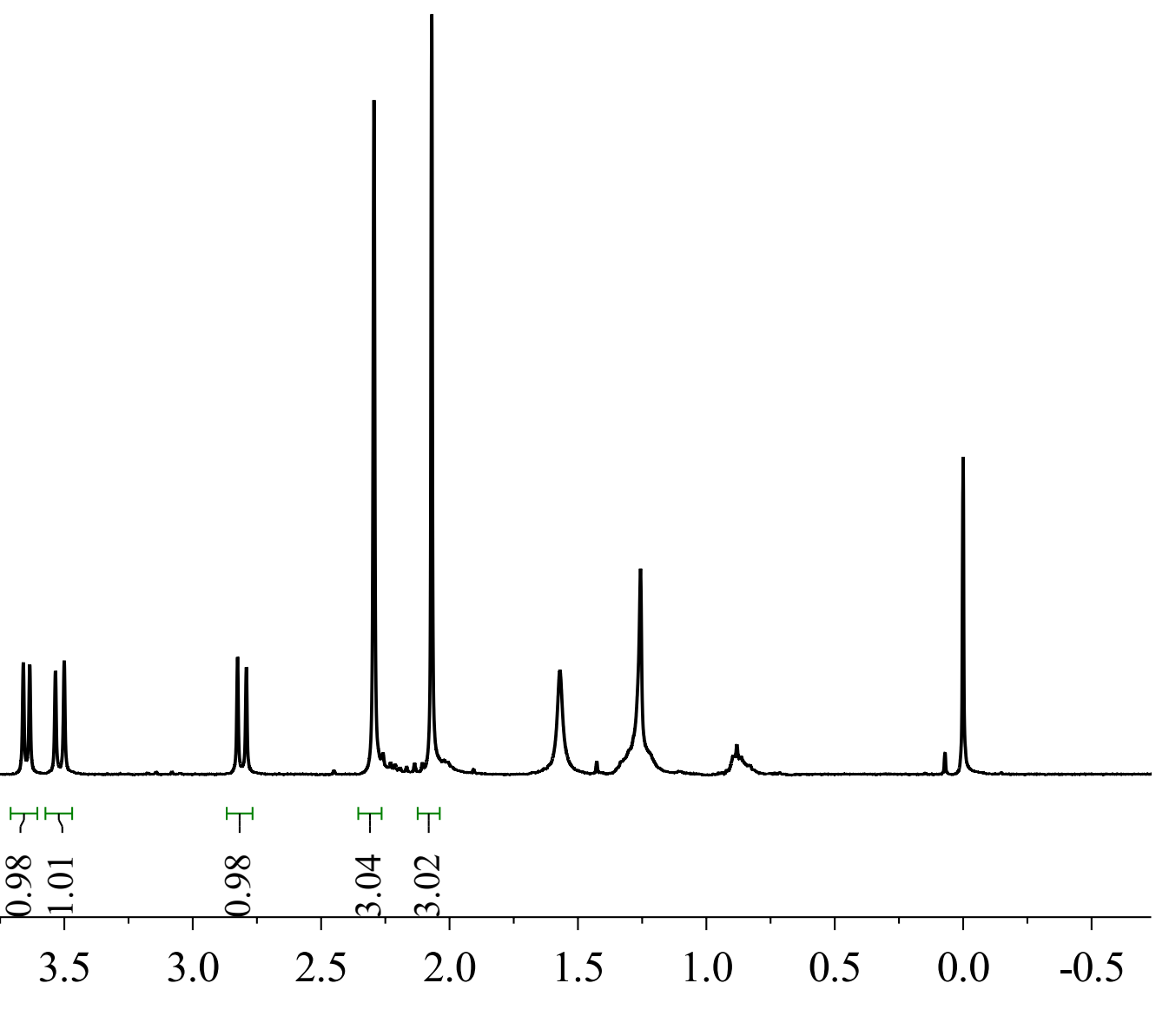



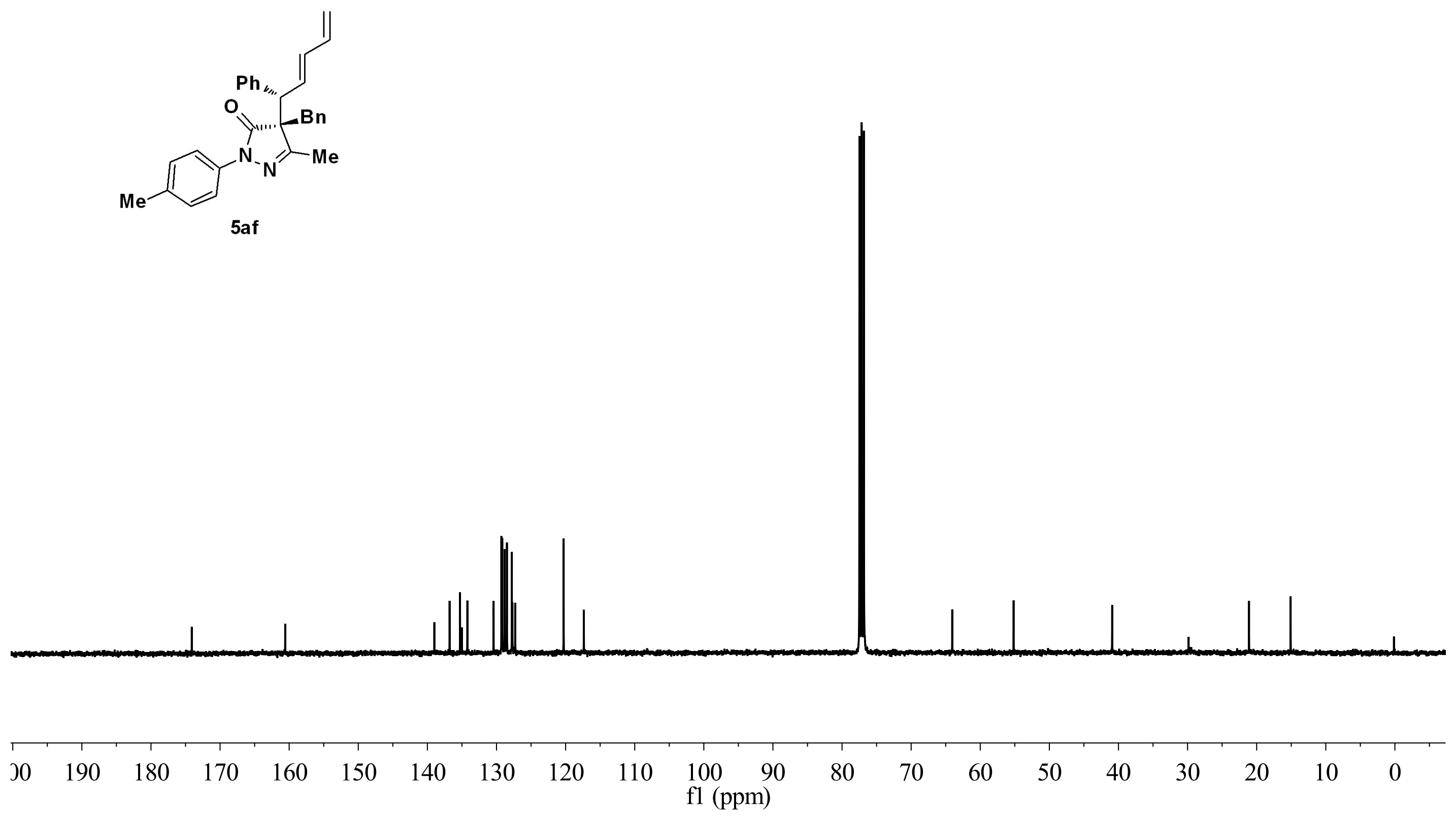

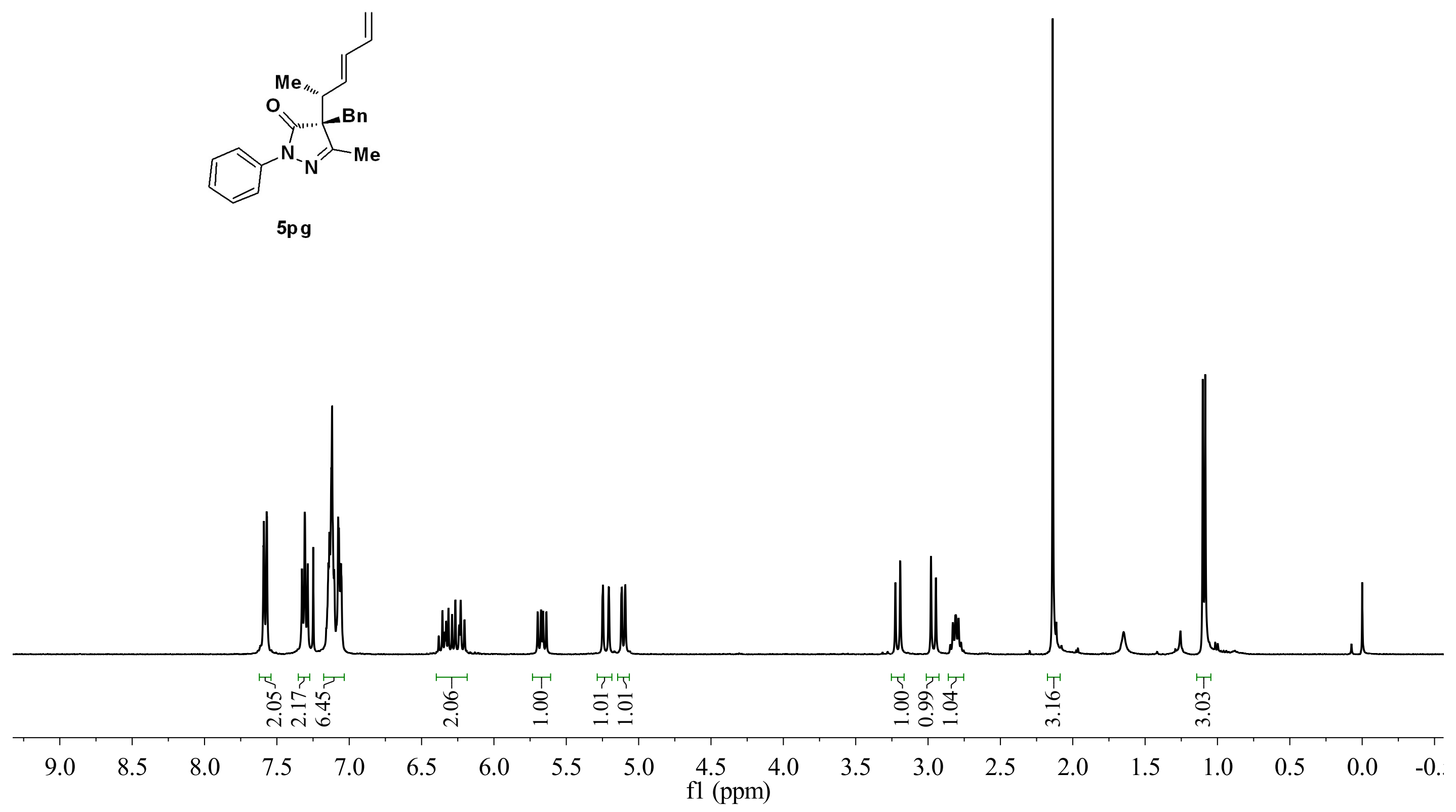


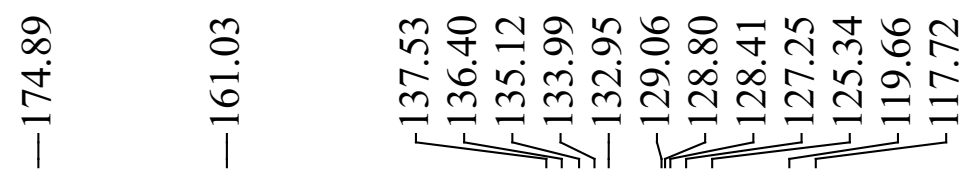

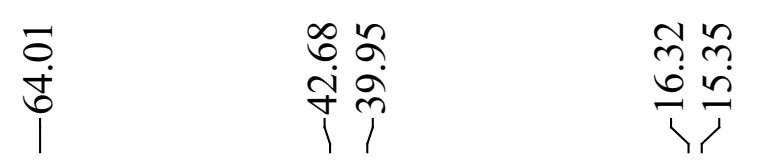
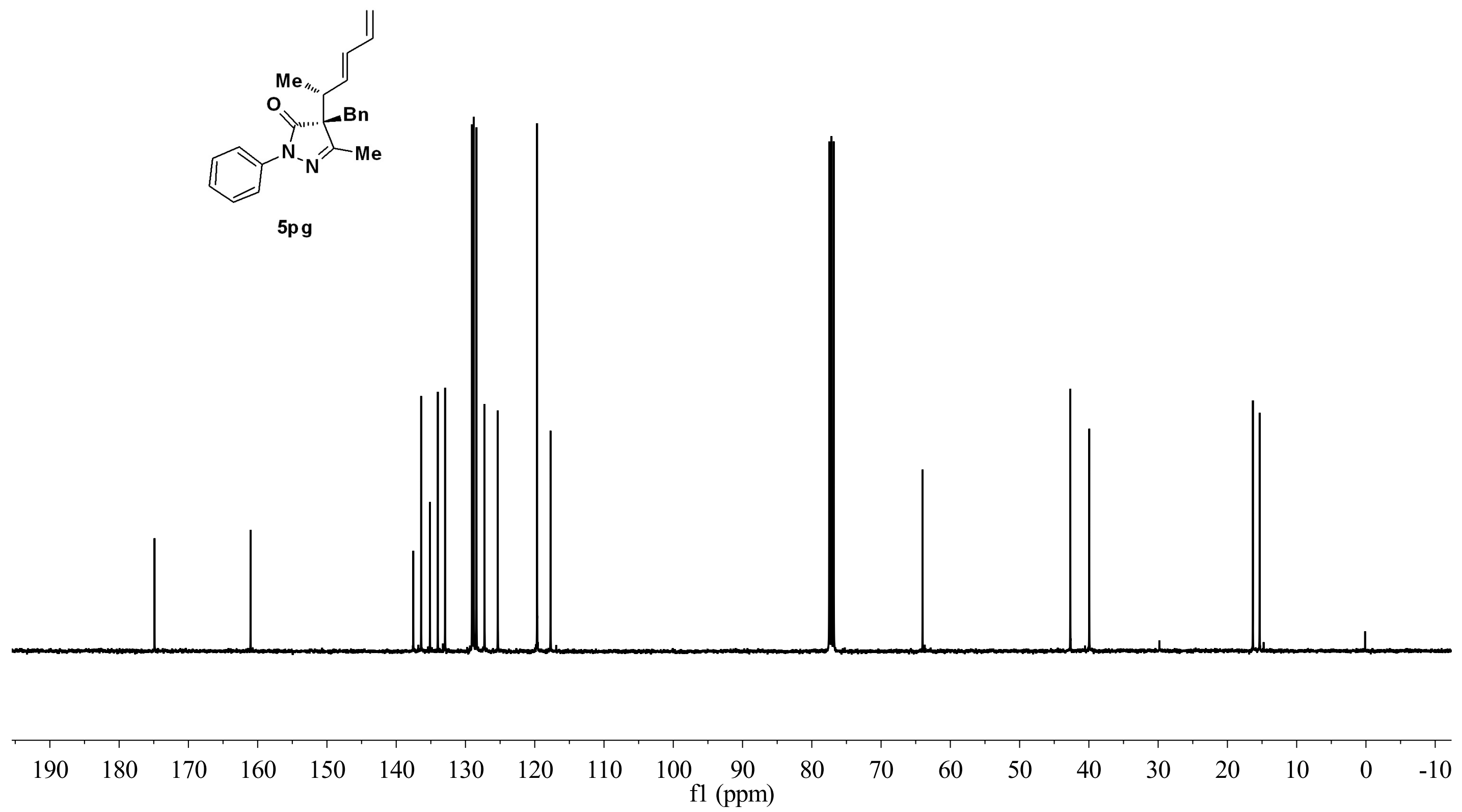


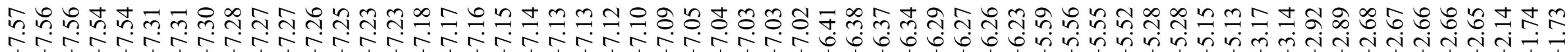<smiles>C=C/C=C/[C@H](CCc1ccccc1)[C@@]1(Cc2ccccc2)C(=O)N(c2ccccc2)N=C1C</smiles>

$5 p e$

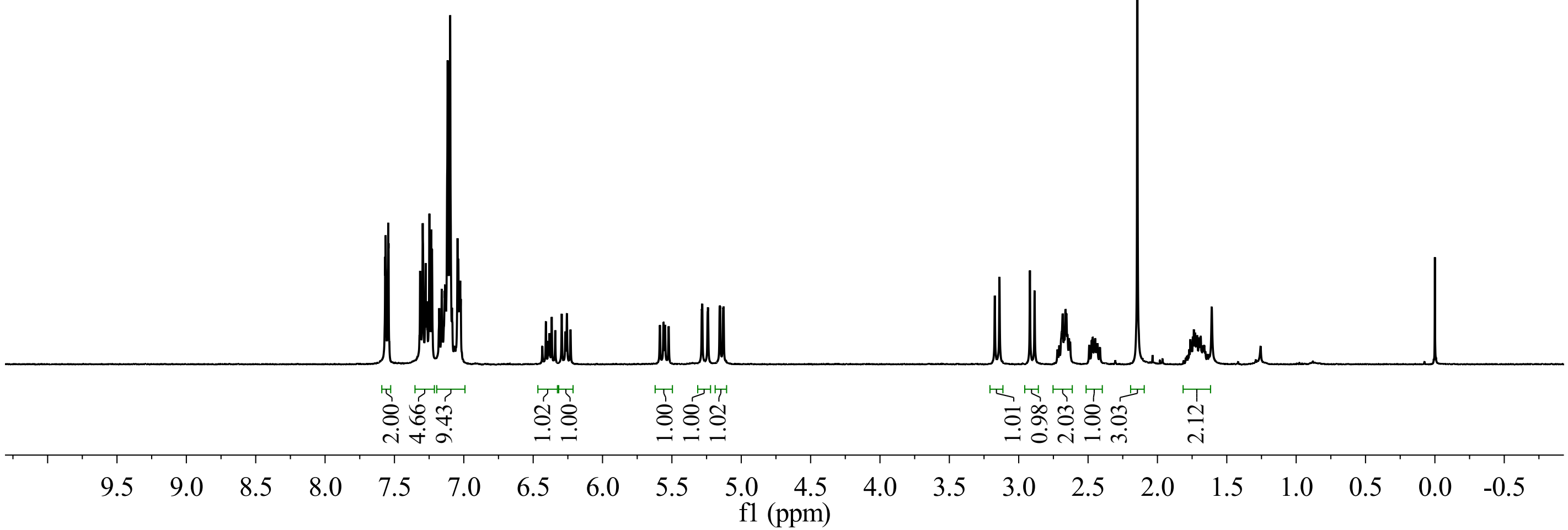



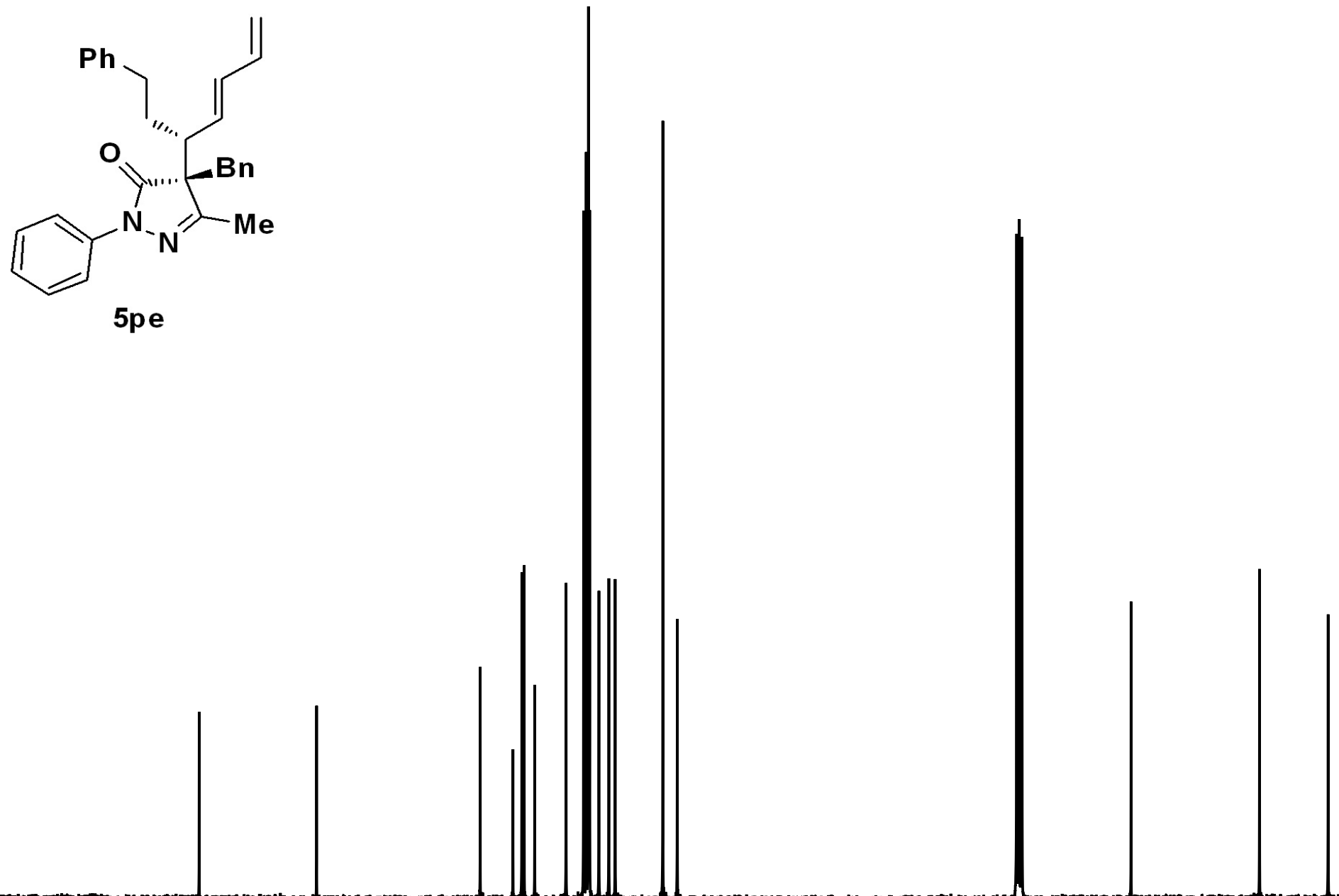

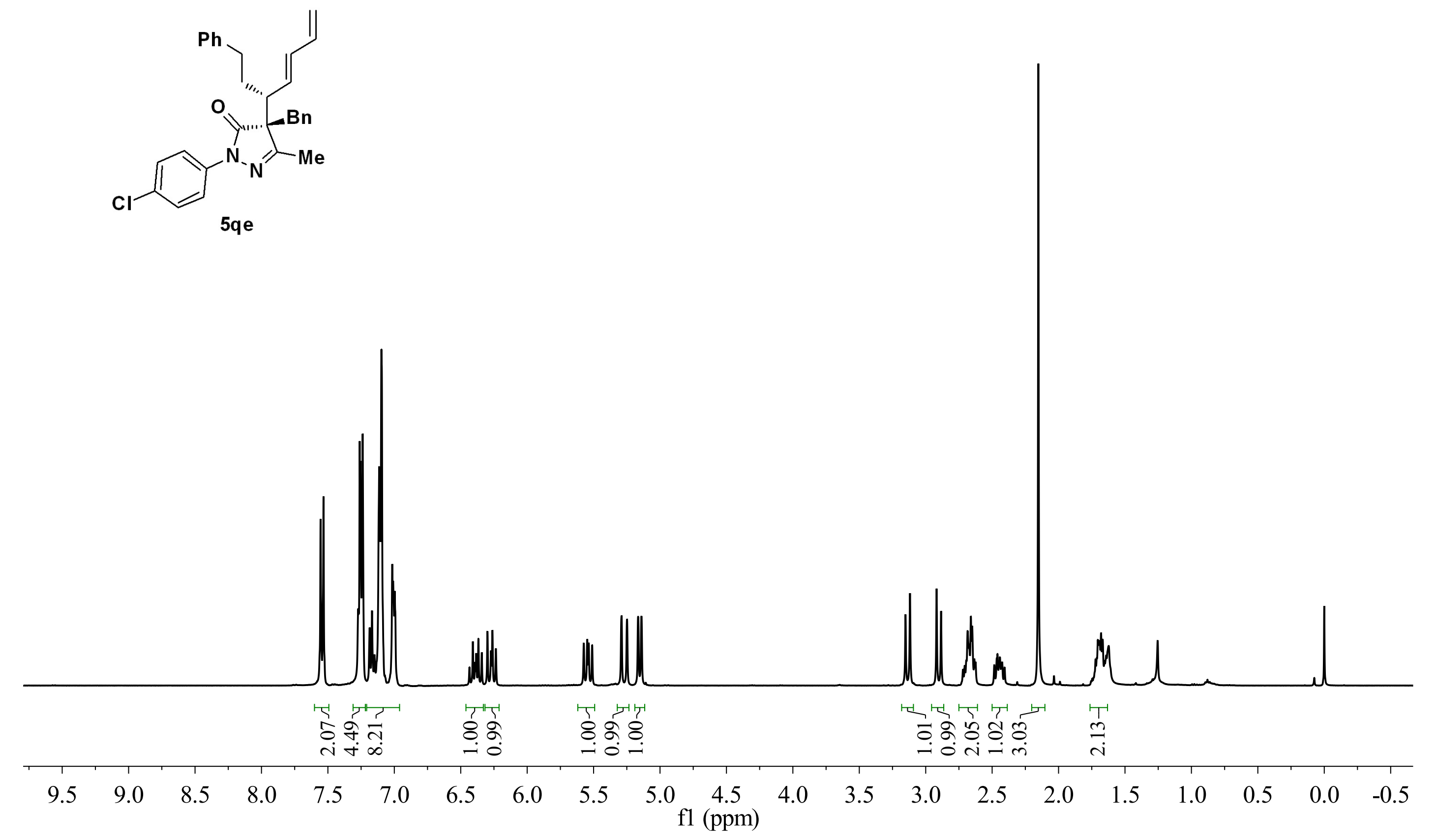


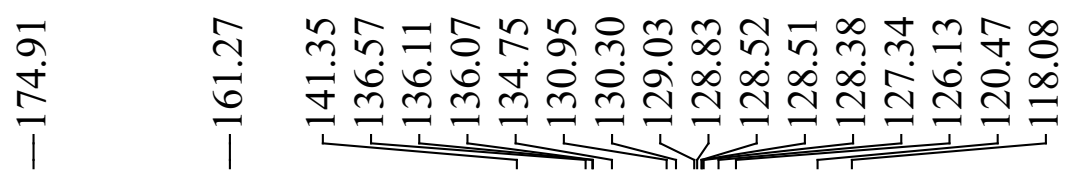

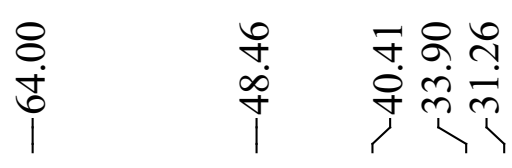

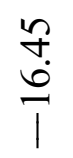
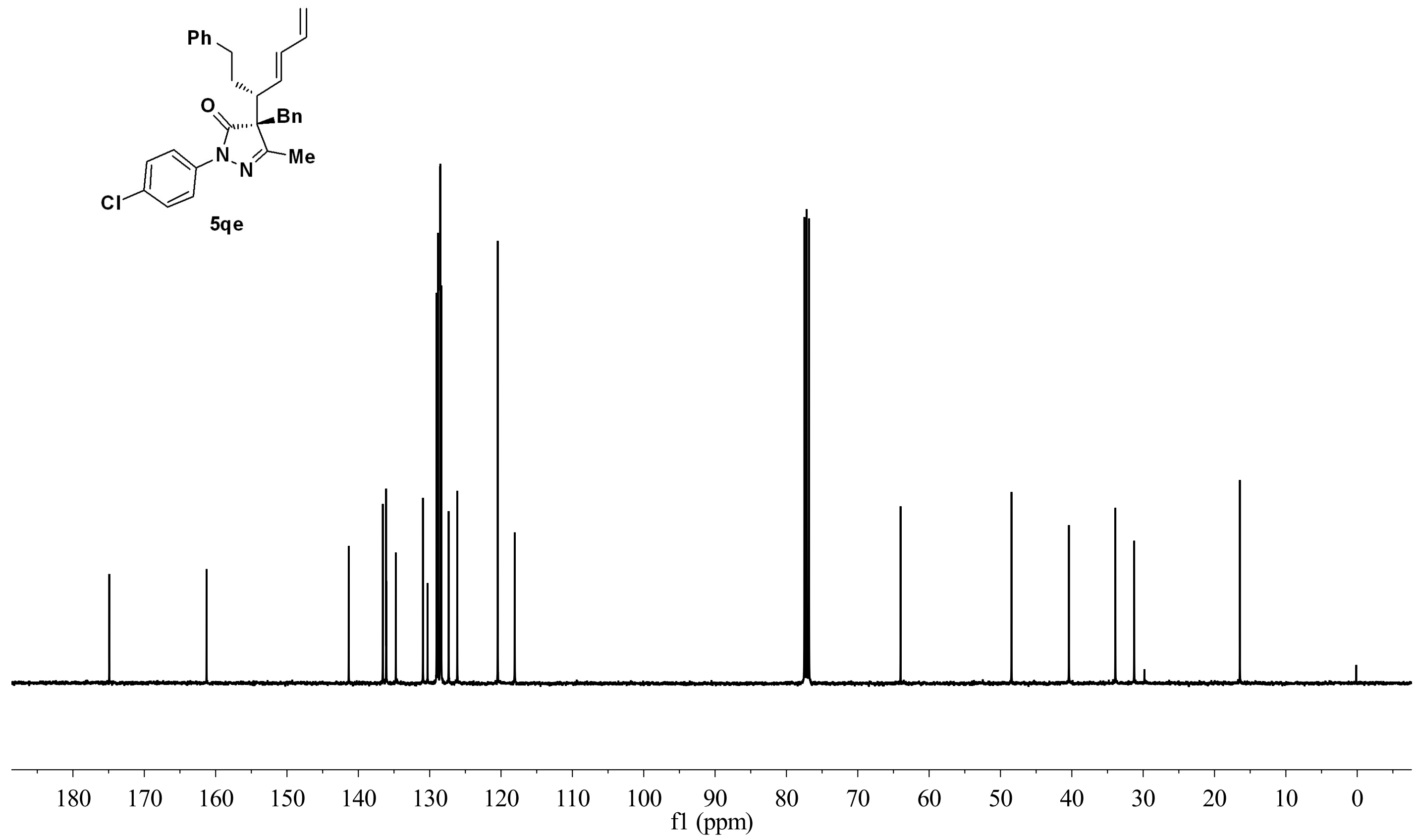


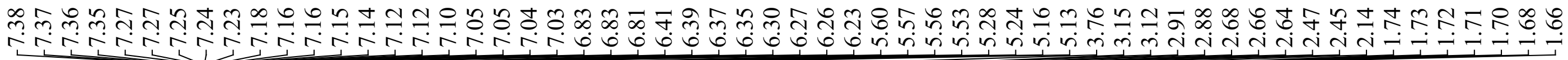<smiles>C=C/C=C/[C@H](CCc1ccccc1)[C@@]1(Cc2ccccc2)C(=O)N(c2ccc(OC)cc2)N=C1C</smiles>

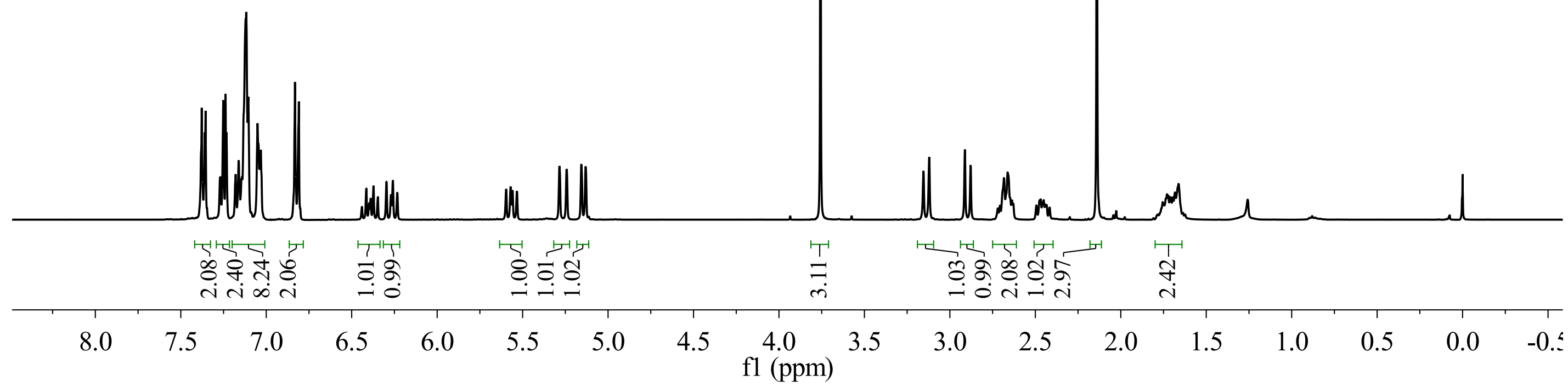




$$
\text { 等 }
$$




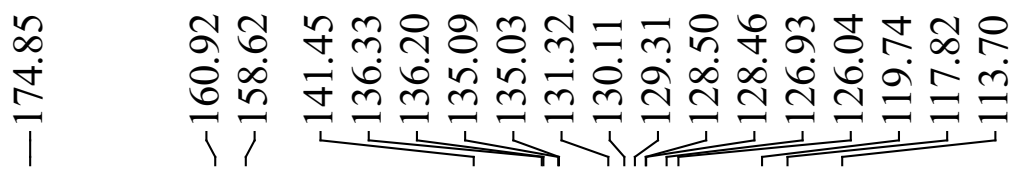

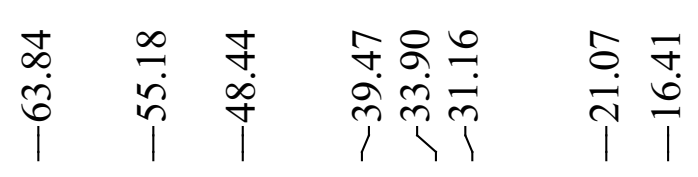
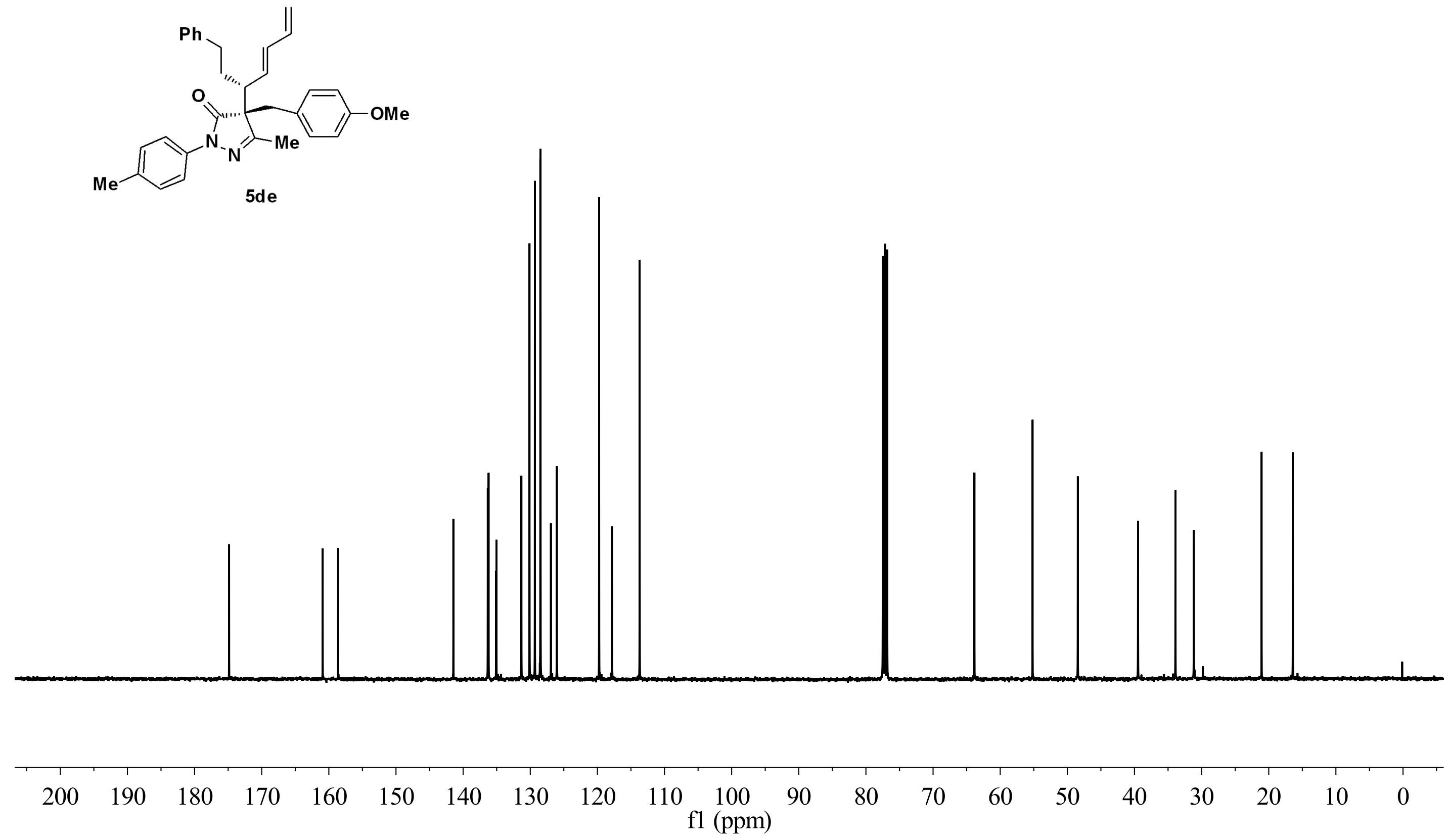

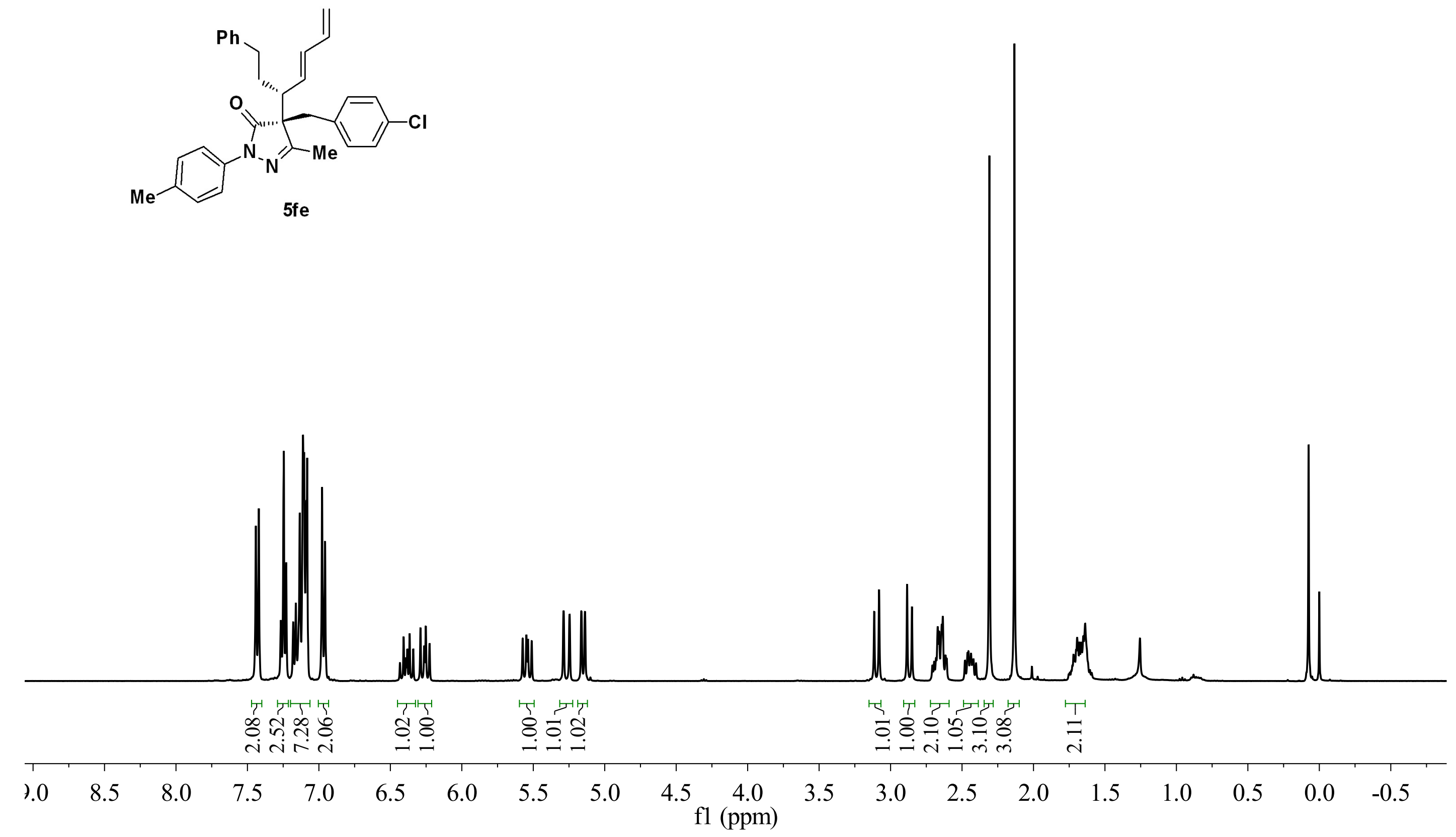


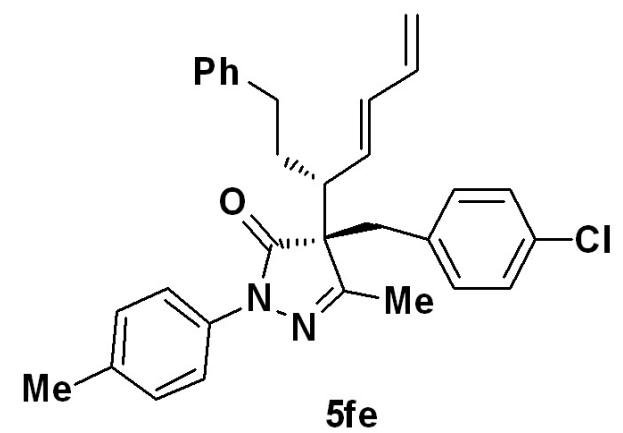



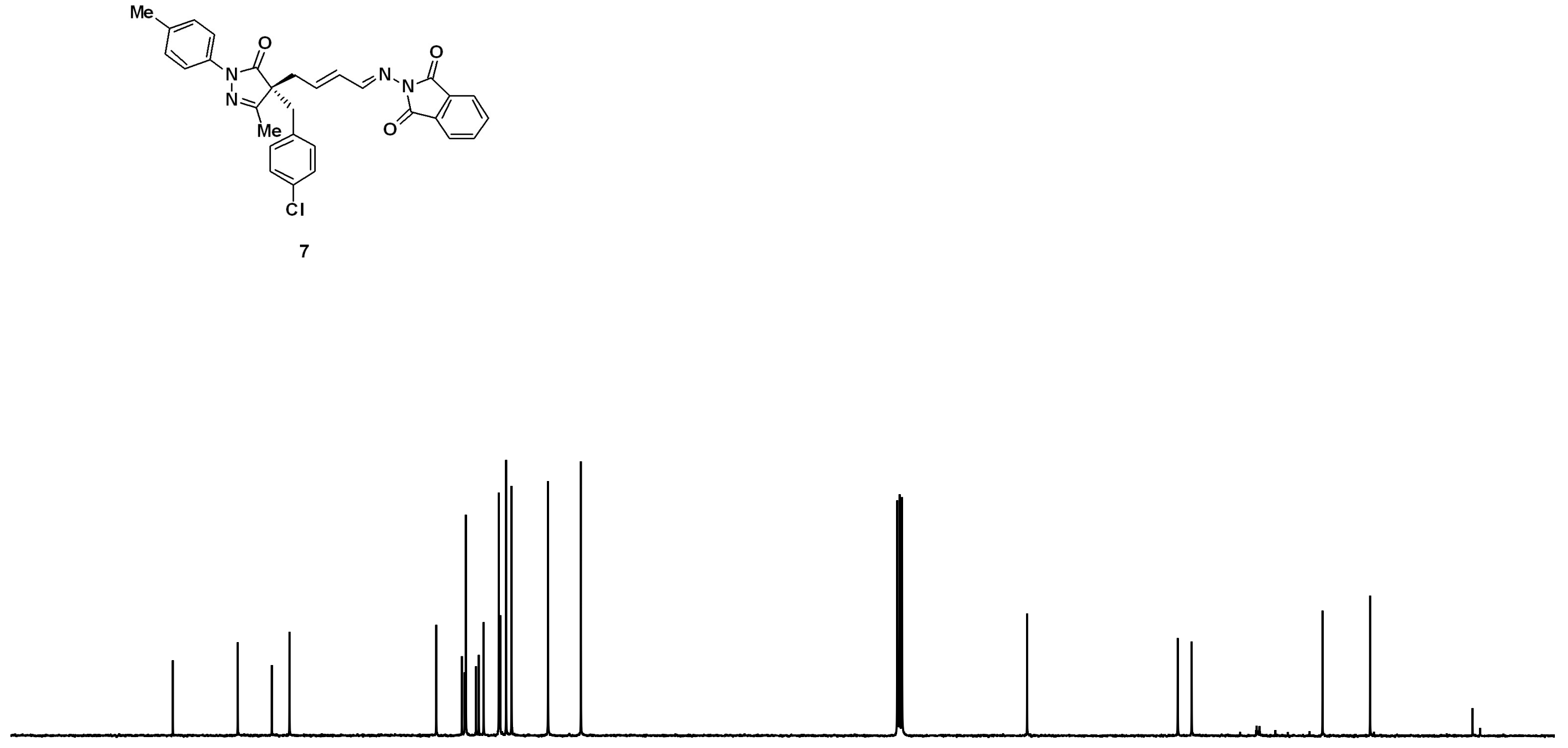

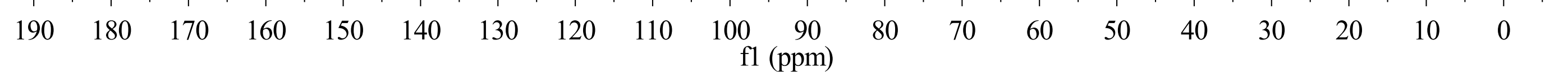


$\mathrm{Me}$

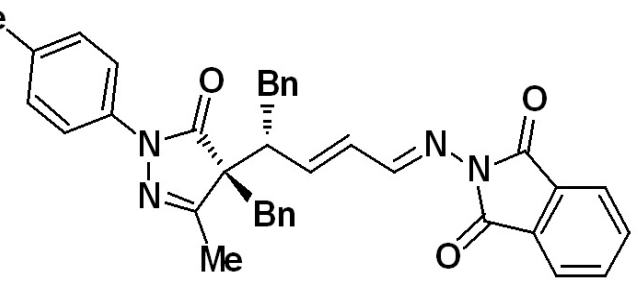

8

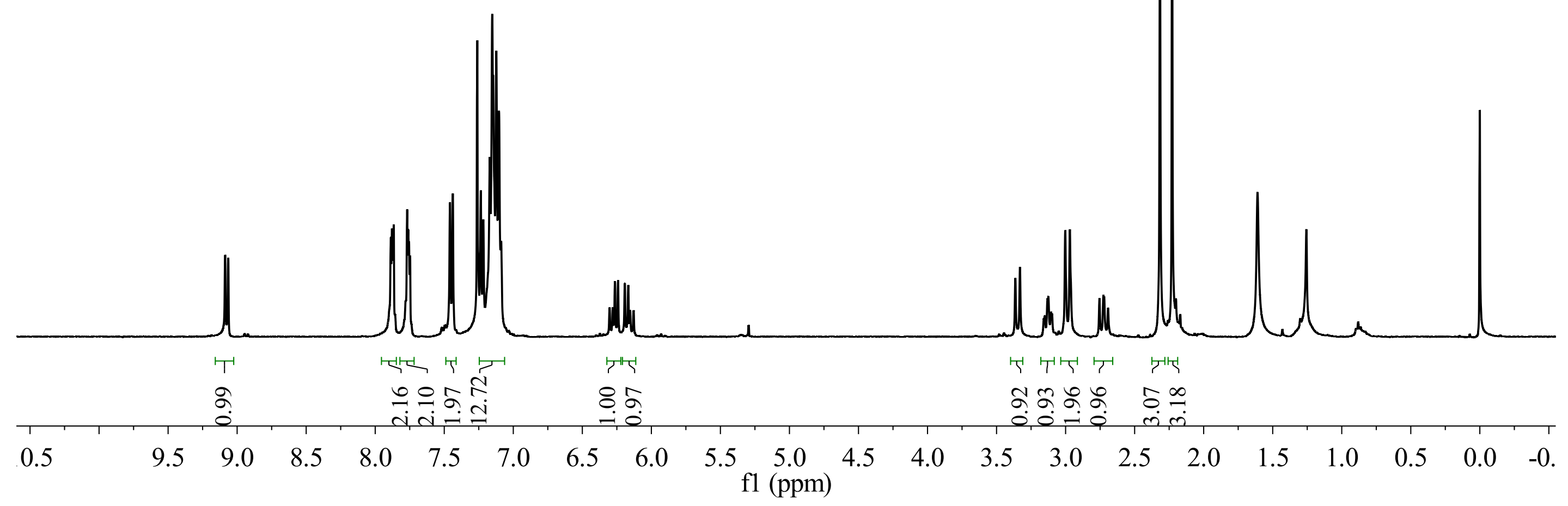




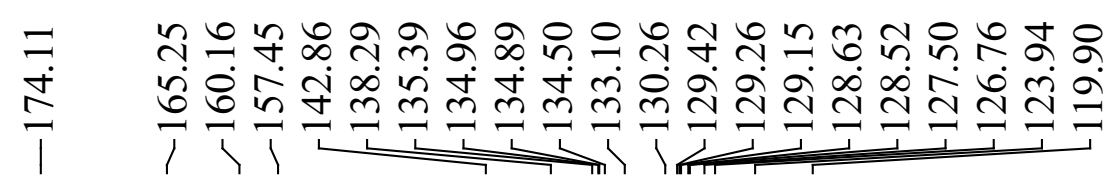

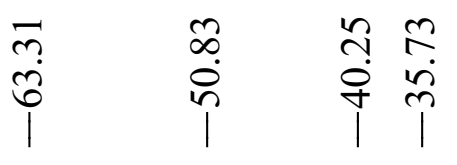

윌<smiles>CC1=NN(c2ccc(C)cc2)C(=O)[C@]1(Br)[C@@H](/C=C/C=N/N1C(=O)c2ccccc2C1=O)Cc1ccccc1</smiles>

8
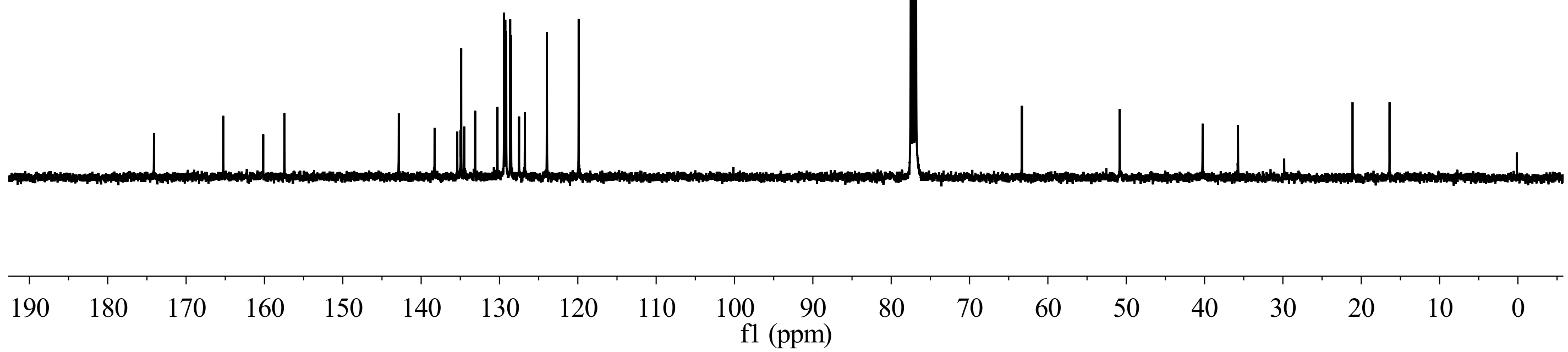
$\mathrm{Me}$<smiles>CC(=O)c1cccc(/C=C/C=C/C[C@@]2(Cc3ccccc3)C(=O)N(c3ccc(C)cc3)N=C2C)c1</smiles>

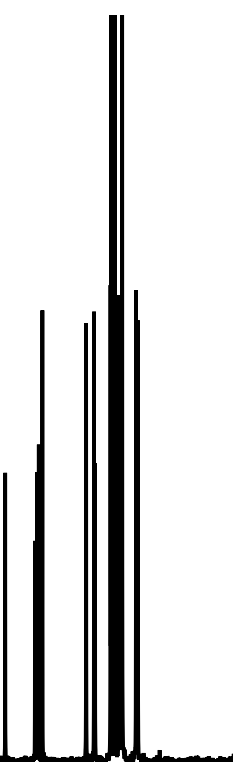


$\mathrm{Me}$
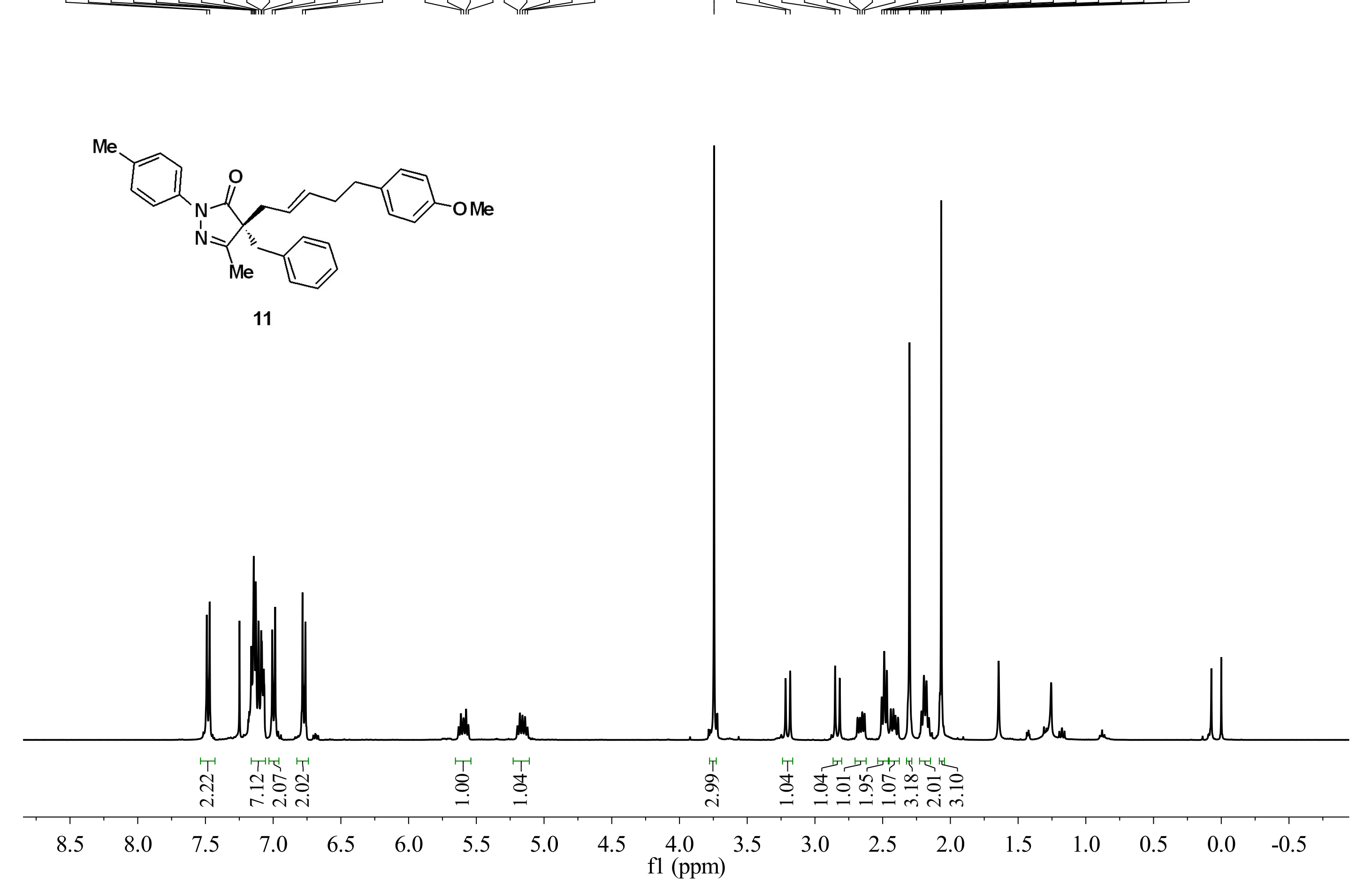
$\mathrm{Me}$<smiles>COc1ccc(CC/C=C/C[C@@]2(Cc3ccccc3)C(=O)N(c3ccc(C)cc3)N=C2C)cc1</smiles>

11

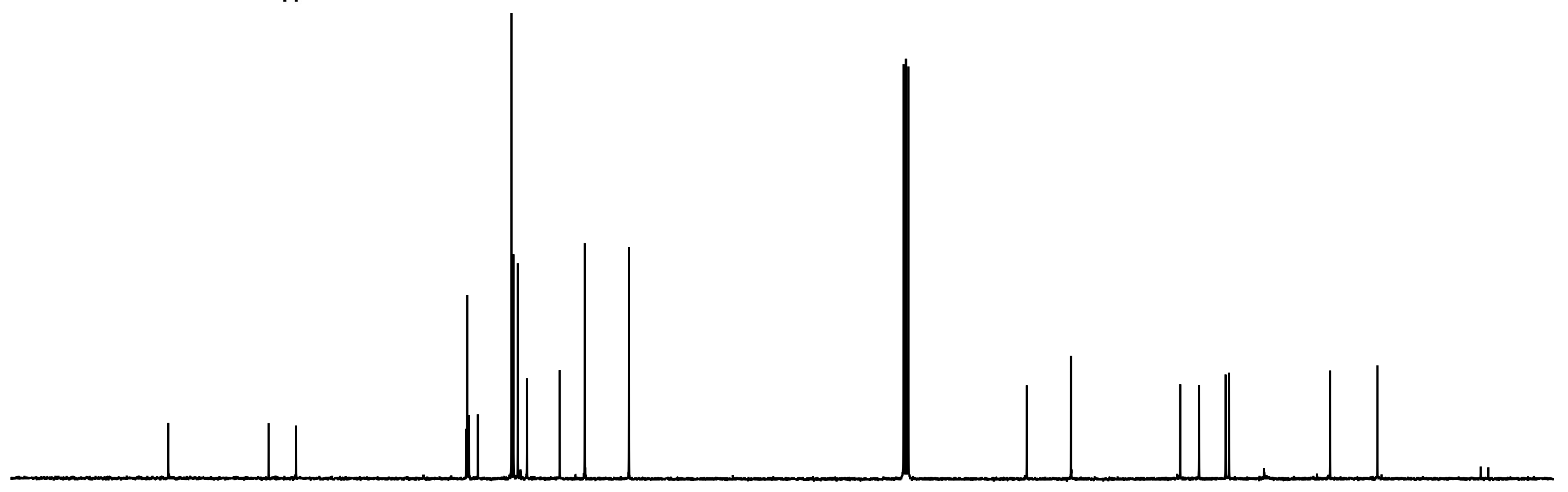




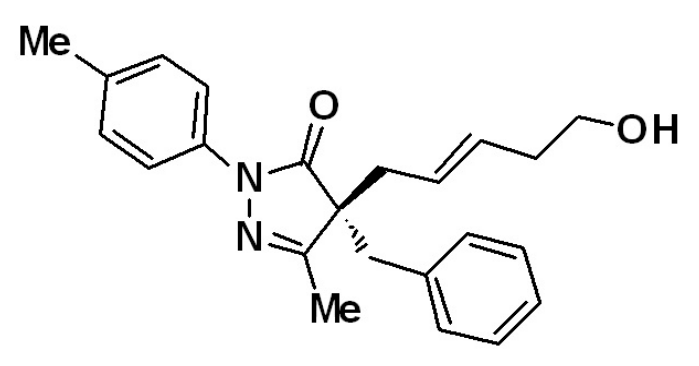

12

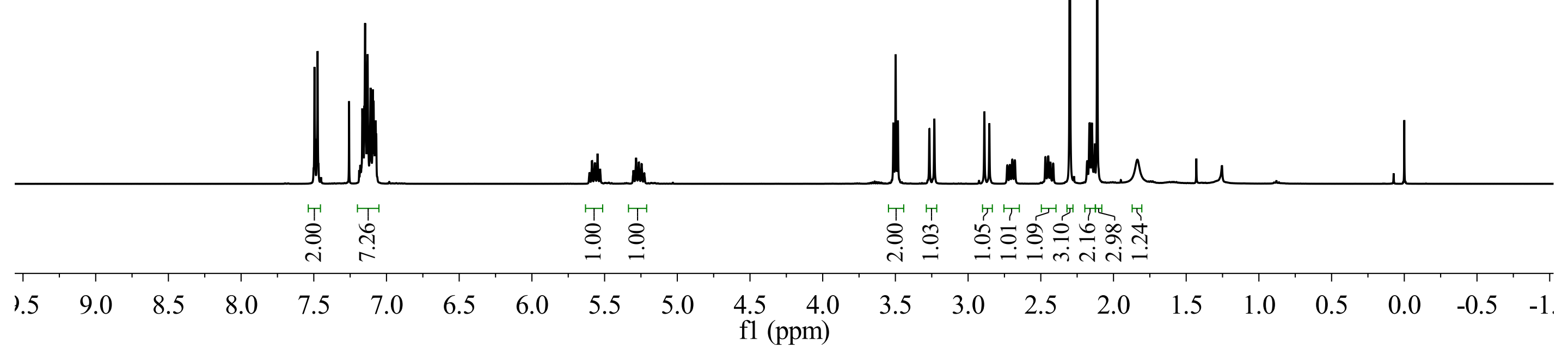


$\mathrm{Me}$

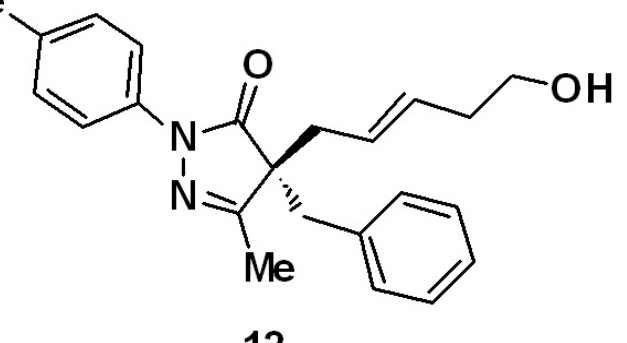

12

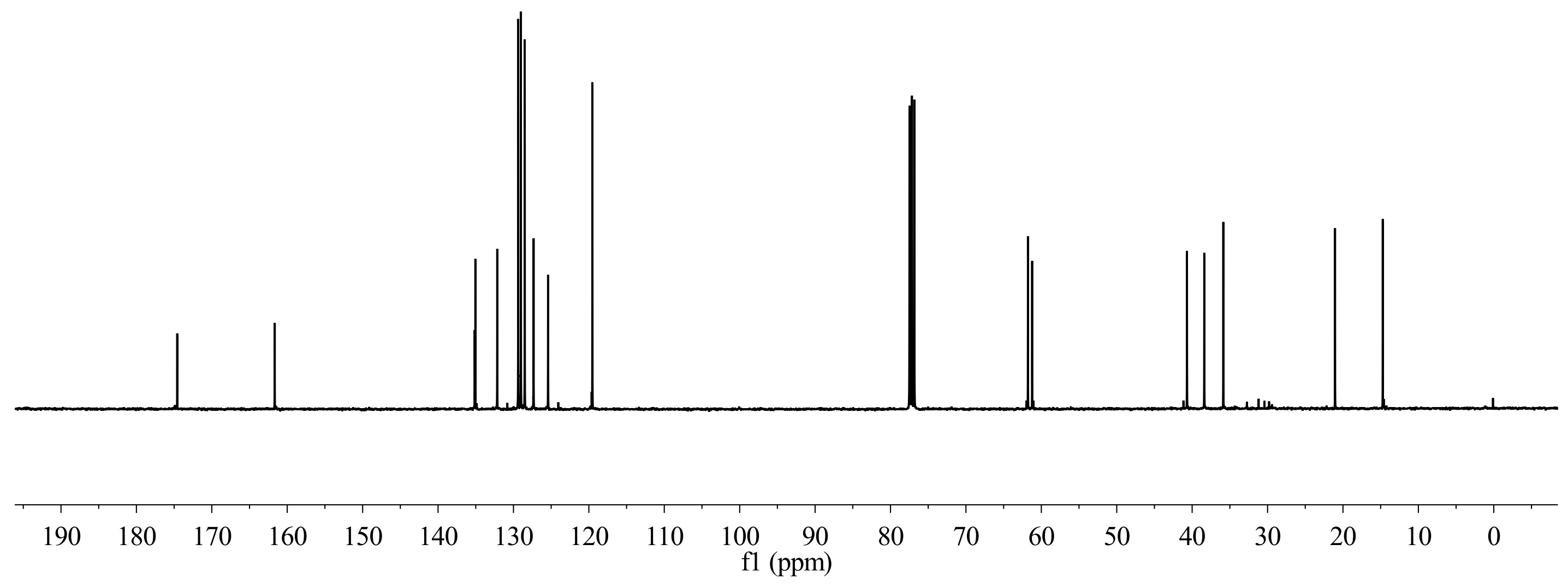




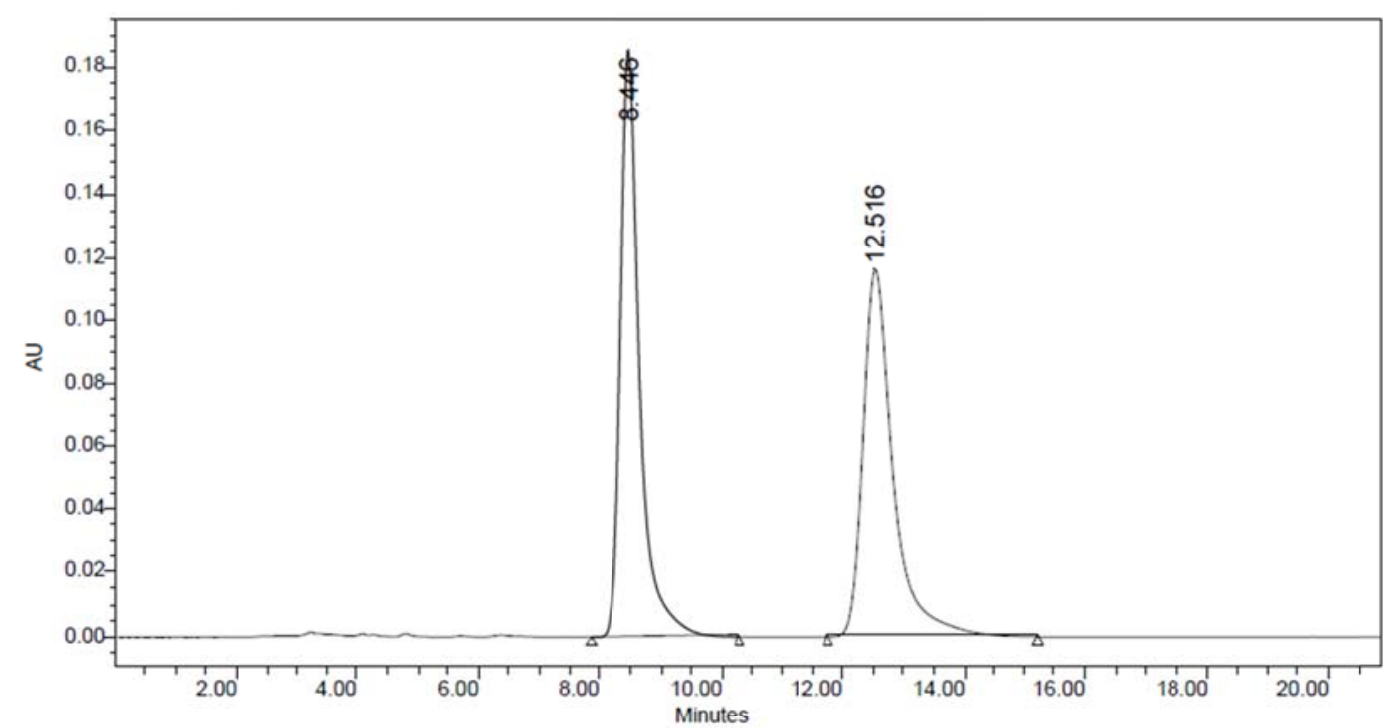

\begin{tabular}{|c|c|c|c|c|c|}
\hline & $\begin{array}{c}\text { RT } \\
(\mathrm{min})\end{array}$ & $\begin{array}{c}\text { Area } \\
\left(\mathrm{V}^{*} \text { sec }\right)\end{array}$ & \% Area & $\begin{array}{c}\text { Height } \\
(\mathrm{V})\end{array}$ & $\begin{array}{c}\% \\
\text { Height }\end{array}$ \\
\hline 1 & 8.446 & 4099792 & 50.44 & 185760 & 61.45 \\
\hline 2 & 12.516 & 4027601 & 49.56 & 116518 & 38.55 \\
\hline
\end{tabular}

Me<smiles></smiles>

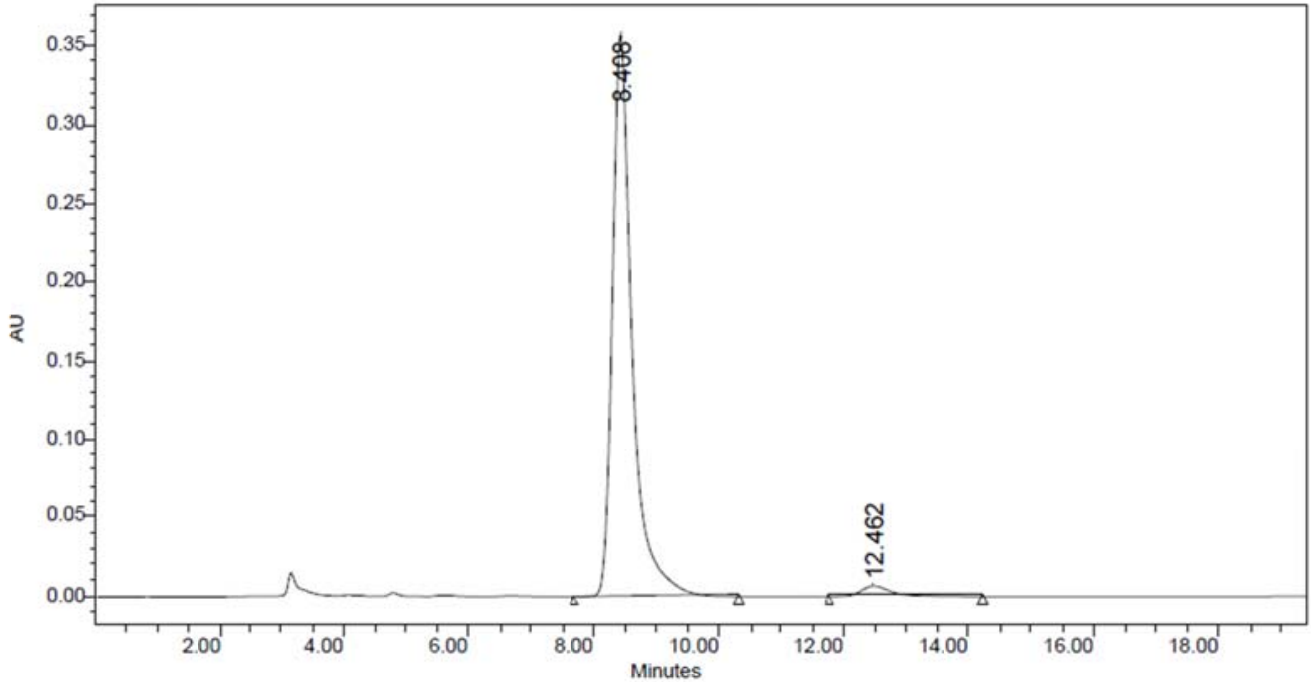

\begin{tabular}{|c|c|c|r|r|r|}
\hline & $\begin{array}{c}\text { RT } \\
(\mathrm{min})\end{array}$ & $\begin{array}{c}\text { Area } \\
\left(\mathbb{V}^{*} \text { sec }\right)\end{array}$ & \% Area & $\begin{array}{c}\text { Height } \\
(\mathrm{V})\end{array}$ & $\begin{array}{c}\% \\
\text { Height }\end{array}$ \\
\hline 1 & 8.408 & 7815364 & 97.13 & 358434 & 98.14 \\
\hline 2 & 12.462 & 230608 & 2.87 & 6803 & 1.86 \\
\hline
\end{tabular}




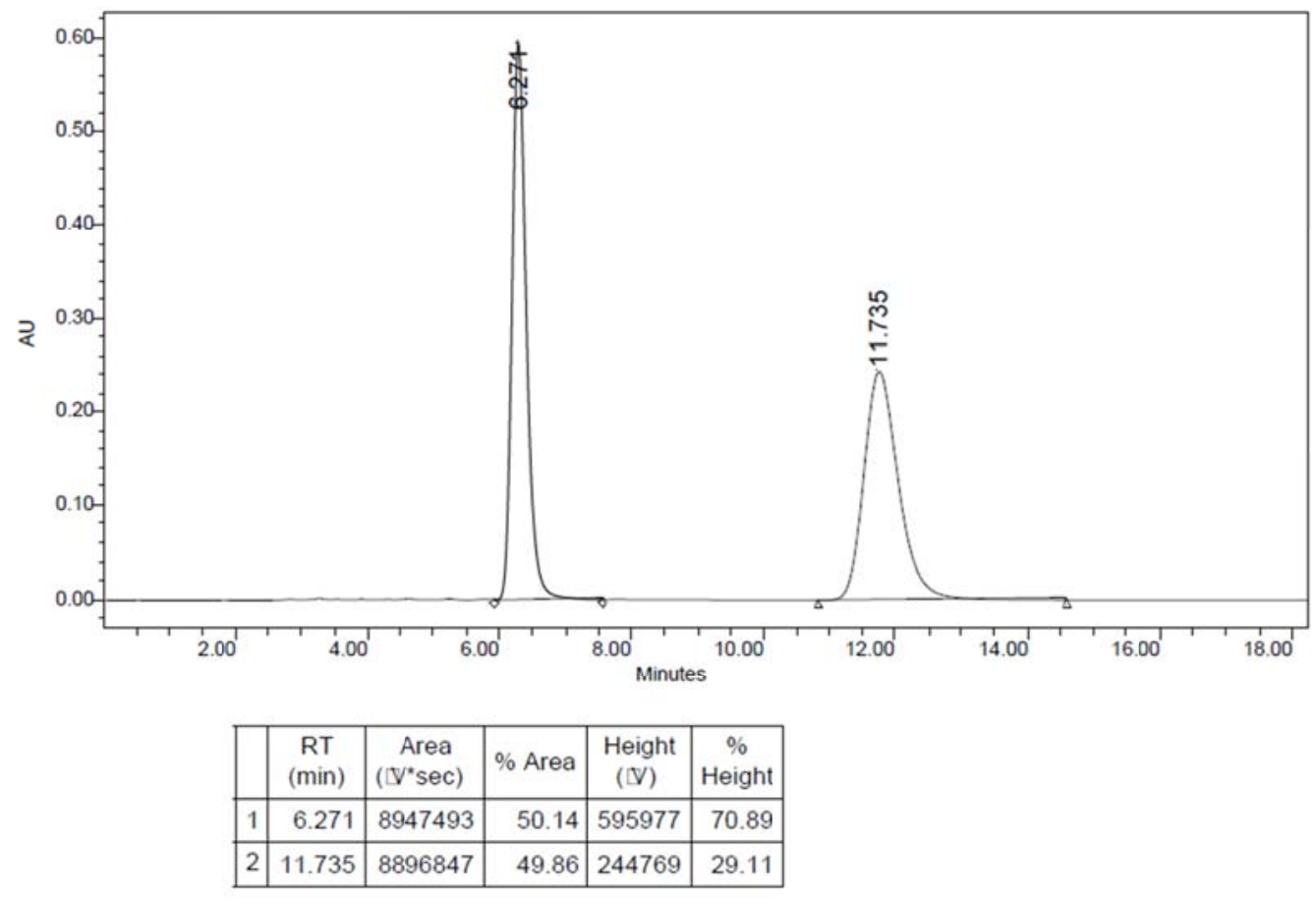

$\mathrm{Me}$
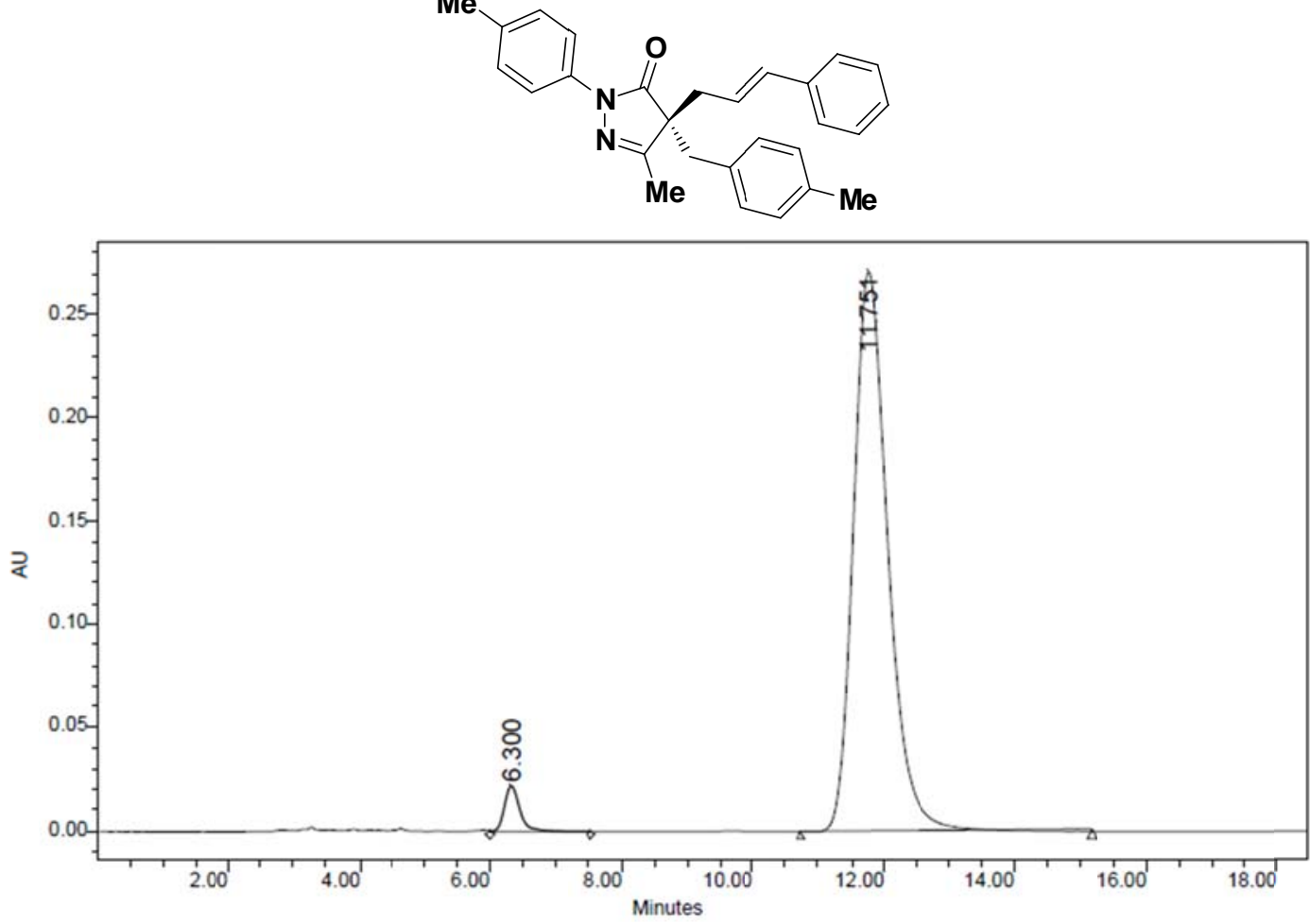

\begin{tabular}{|l|c|c|r|c|r|}
\hline & $\begin{array}{c}\mathrm{RT} \\
(\mathrm{min})\end{array}$ & $\begin{array}{c}\text { Area } \\
\left(\mathrm{V}^{*} \mathrm{sec}\right)\end{array}$ & $\%$ Area & $\begin{array}{c}\text { Height } \\
(\mathrm{V})\end{array}$ & $\begin{array}{c}\% \\
\text { Height }\end{array}$ \\
\hline 1 & 6.300 & 365402 & 3.56 & 22037 & 7.51 \\
\hline 2 & 11.751 & 9912567 & 96.44 & 271559 & 92.49 \\
\hline
\end{tabular}




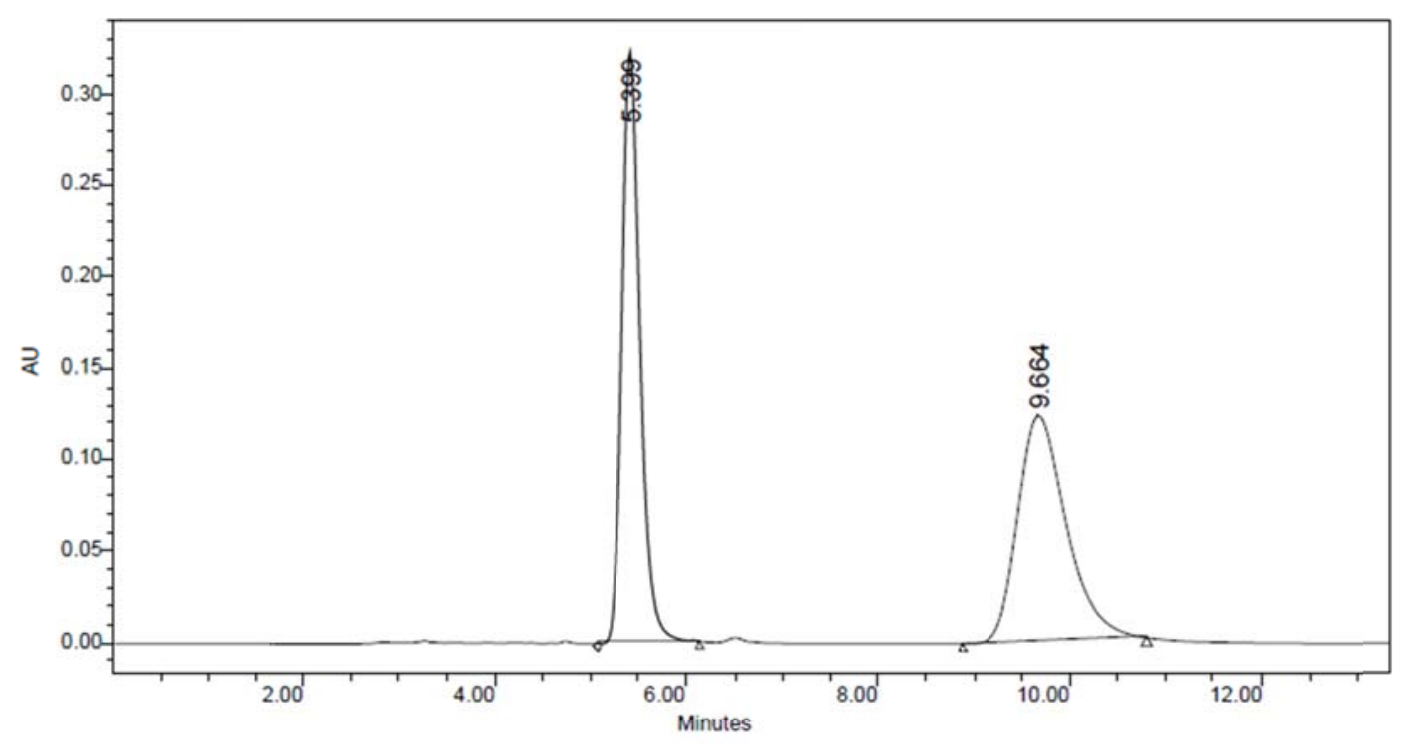

\begin{tabular}{|c|c|c|c|c|c|}
\hline & $\begin{array}{c}\text { RT } \\
(\min )\end{array}$ & $\begin{array}{c}\text { Area } \\
\left(V^{*} \text { sec }\right)\end{array}$ & $\%$ Area & $\begin{array}{c}\text { Height } \\
(\mathrm{V})\end{array}$ & $\begin{array}{c}\% \\
\text { Height }\end{array}$ \\
\hline 1 & 5.399 & 4256219 & 49.43 & 322910 & 72.30 \\
\hline 2 & 9.664 & 4354977 & 50.57 & 123709 & 27.70 \\
\hline
\end{tabular}

Me
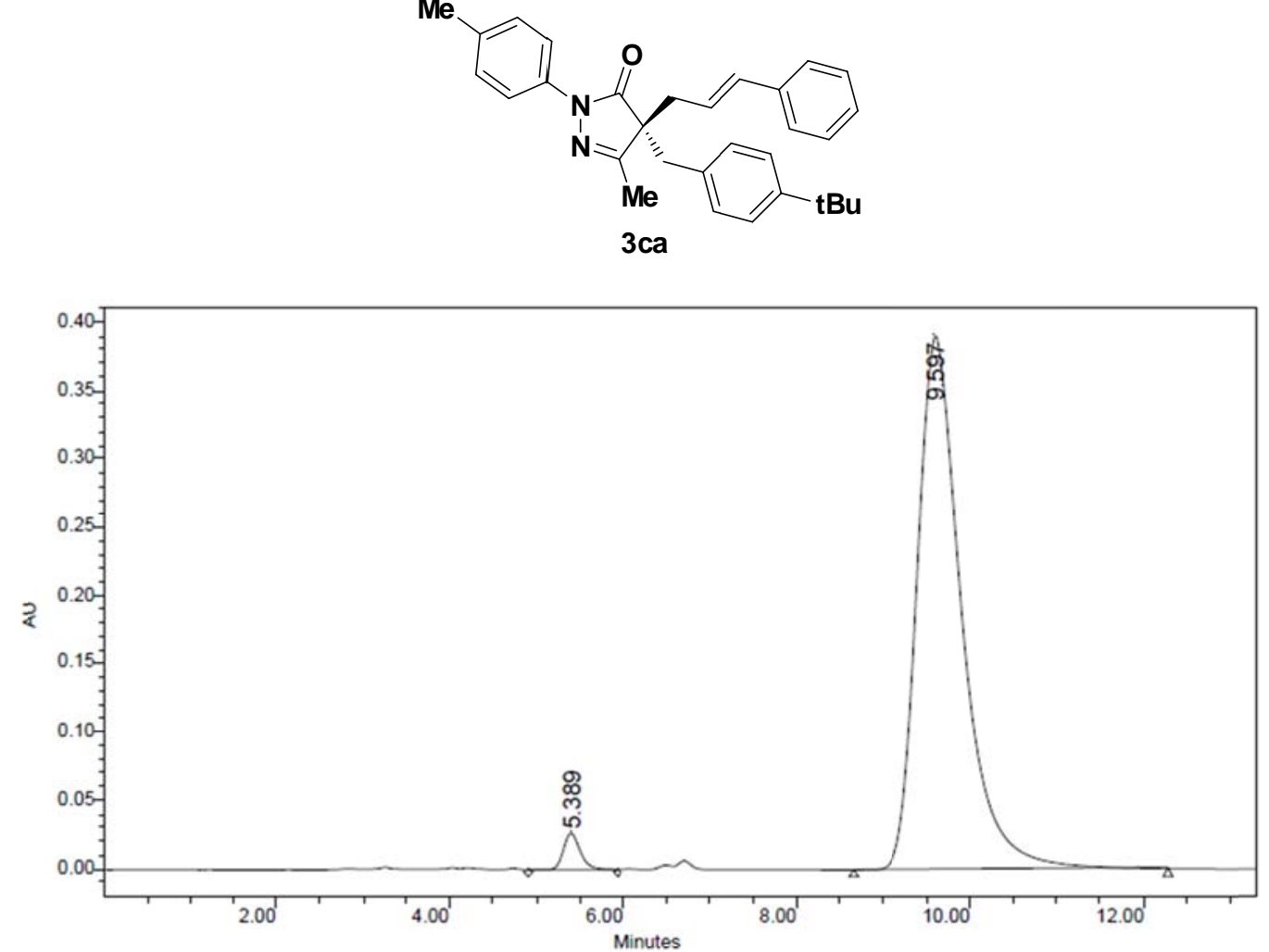

\begin{tabular}{|c|c|c|r|r|r|}
\hline & $\begin{array}{c}\text { RT } \\
(\mathrm{min})\end{array}$ & $\begin{array}{c}\text { Area } \\
\left(\mathrm{V}^{*} \mathrm{sec}\right)\end{array}$ & $\%$ Area & $\begin{array}{c}\text { Height } \\
(\mathrm{V})\end{array}$ & $\begin{array}{c}\% \\
\text { Height }\end{array}$ \\
\hline 1 & 5.389 & 361829 & 2.55 & 26689 & 6.40 \\
\hline 2 & 9.597 & 13842410 & 97.45 & 390476 & 93.60 \\
\hline
\end{tabular}




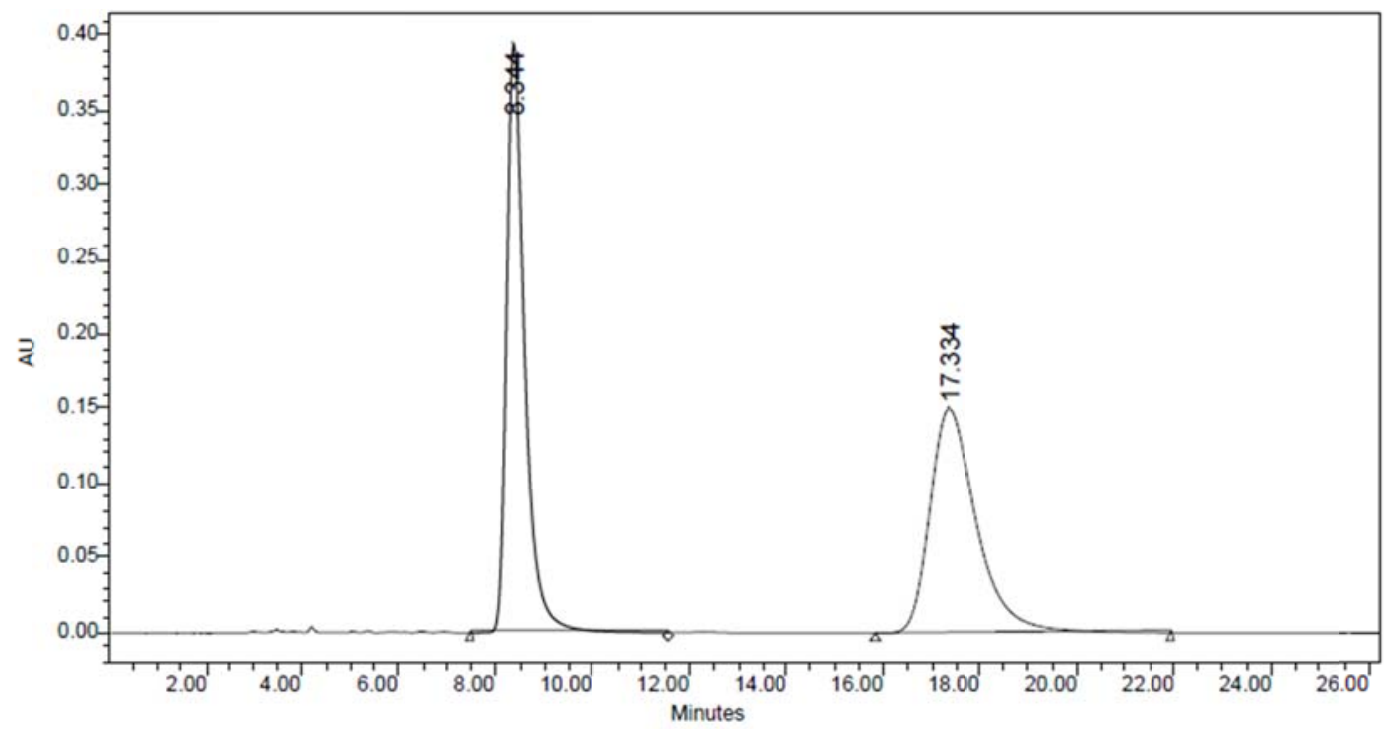

\begin{tabular}{|c|c|c|c|c|c|}
\hline & $\begin{array}{c}\text { RT } \\
(\mathrm{min})\end{array}$ & $\begin{array}{c}\text { Area } \\
\left(\mathrm{V}^{*} \mathrm{sec}\right)\end{array}$ & $\%$ Area & $\begin{array}{c}\text { Height } \\
(\mathrm{V})\end{array}$ & $\begin{array}{c}\% \\
\text { Height }\end{array}$ \\
\hline 1 & 8.344 & 10235186 & 50.20 & 394758 & 72.40 \\
\hline 2 & 17.334 & 10153705 & 49.80 & 150496 & 27.60 \\
\hline
\end{tabular}
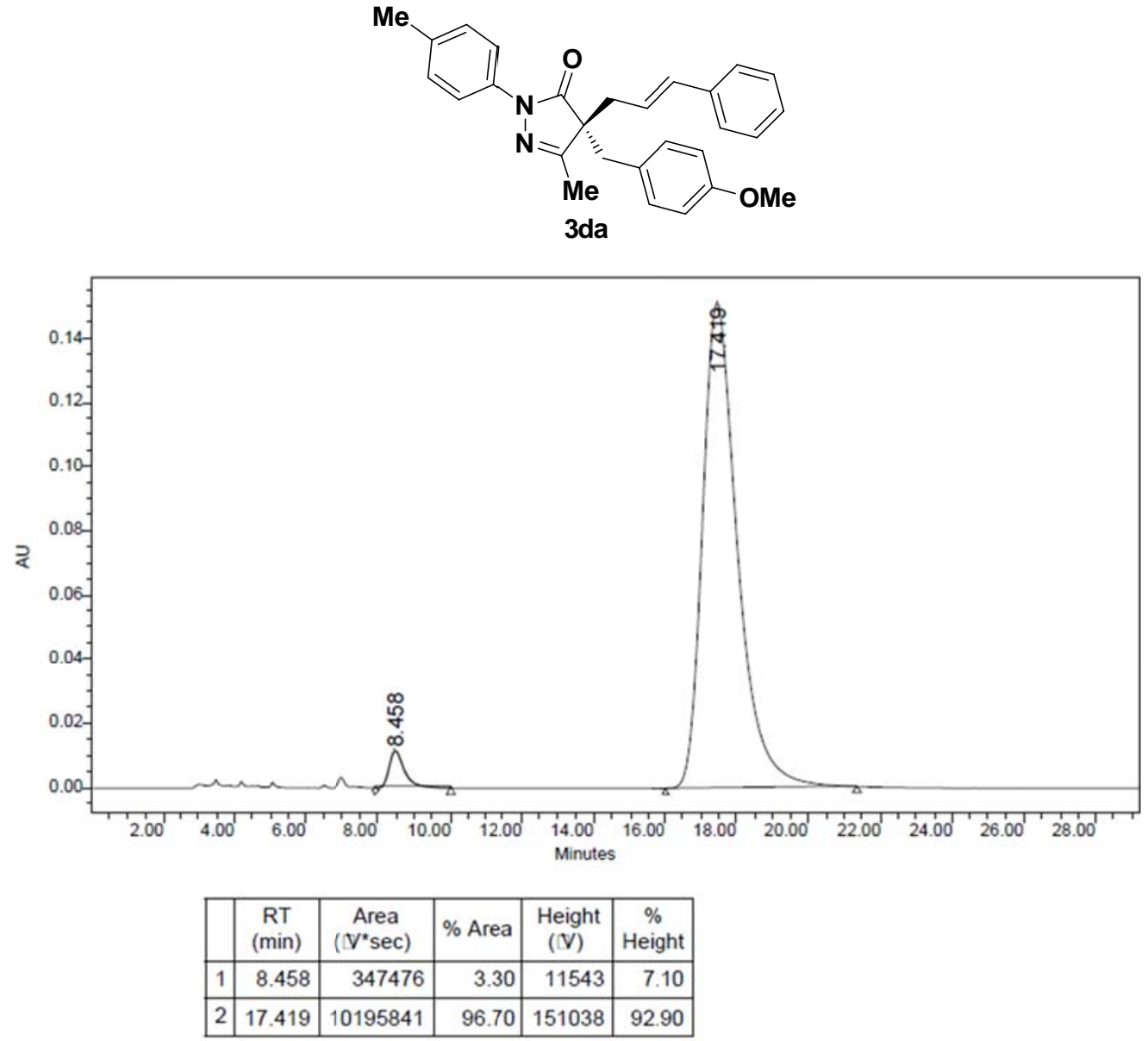


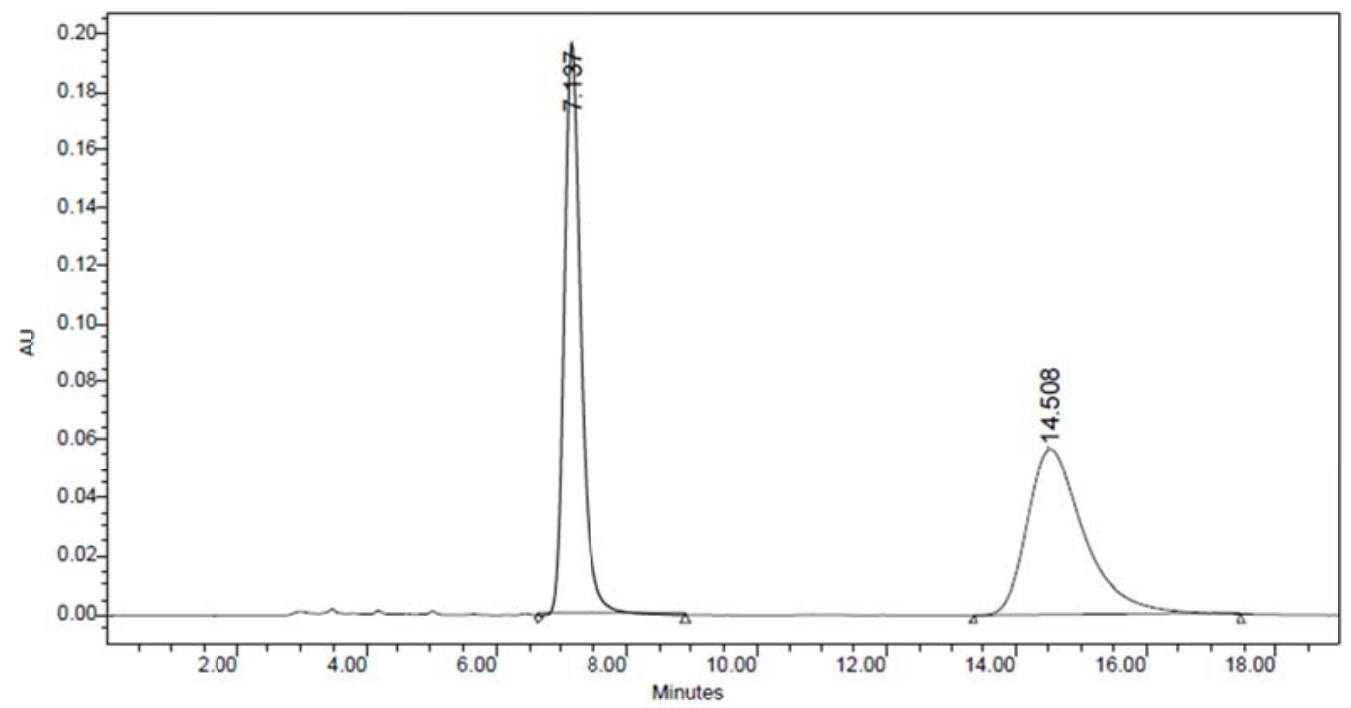

\begin{tabular}{|c|c|c|c|c|c|}
\hline & $\begin{array}{c}\text { RT } \\
(\mathrm{min})\end{array}$ & $\begin{array}{c}\text { Area } \\
\left(\mathrm{V}^{*} \mathrm{sec}\right)\end{array}$ & \% Area & $\begin{array}{c}\text { Height } \\
(\mathrm{V})\end{array}$ & $\begin{array}{c}\% \\
\text { Height }\end{array}$ \\
\hline 1 & 7.137 & 3533541 & 50.54 & 196800 & 77.50 \\
\hline 2 & 14.508 & 3458622 & 49.46 & 57136 & 22.50 \\
\hline
\end{tabular}

Me<smiles>CC1=NN(c2ccc(C)cc2)C(=O)C1(C/C=C/c1ccccc1)Cc1ccc(F)cc1</smiles>

3ea

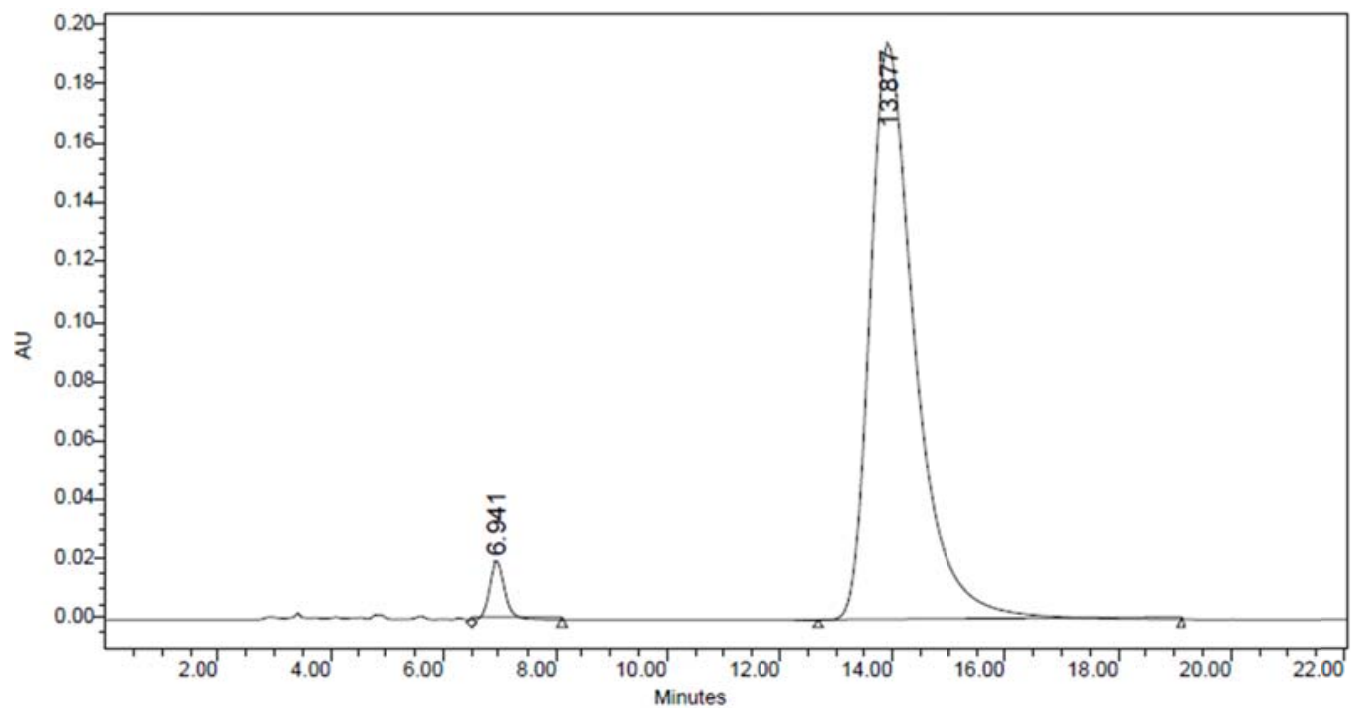

\begin{tabular}{|c|c|r|r|r|r|}
\hline & $\begin{array}{c}\text { RT } \\
(\mathrm{min})\end{array}$ & $\begin{array}{c}\text { Area } \\
\left(\mathrm{V}^{*} \mathrm{sec}\right)\end{array}$ & $\%$ Area & $\begin{array}{c}\text { Height } \\
(\mathrm{V})\end{array}$ & $\begin{array}{c}\% \\
\text { Height }\end{array}$ \\
\hline 1 & 6.941 & 367555 & 3.29 & 19743 & 9.26 \\
\hline 2 & 13.877 & 10803906 & 96.71 & 193549 & 90.74 \\
\hline
\end{tabular}




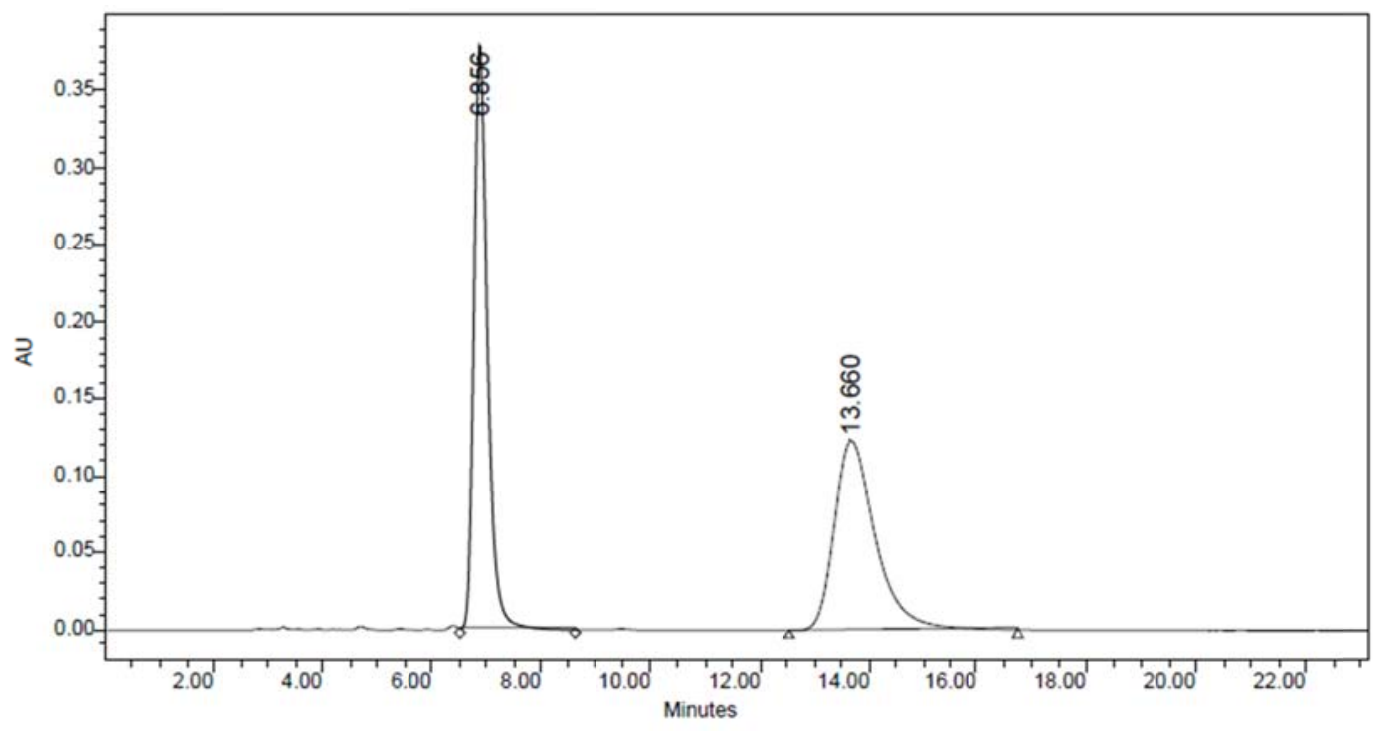

\begin{tabular}{|c|c|c|c|c|c|}
\hline & $\begin{array}{c}\mathrm{RT} \\
(\mathrm{min})\end{array}$ & $\begin{array}{c}\text { Area } \\
\left(\mathrm{V}^{*} \mathrm{sec}\right)\end{array}$ & $\%$ Area & $\begin{array}{c}\text { Height } \\
(\mathrm{V})\end{array}$ & $\begin{array}{c}\% \\
\text { Height }\end{array}$ \\
\hline 1 & 6.856 & 6587942 & 50.42 & 381226 & 75.56 \\
\hline 2 & 13.660 & 6477586 & 49.58 & 123300 & 24.44 \\
\hline
\end{tabular}
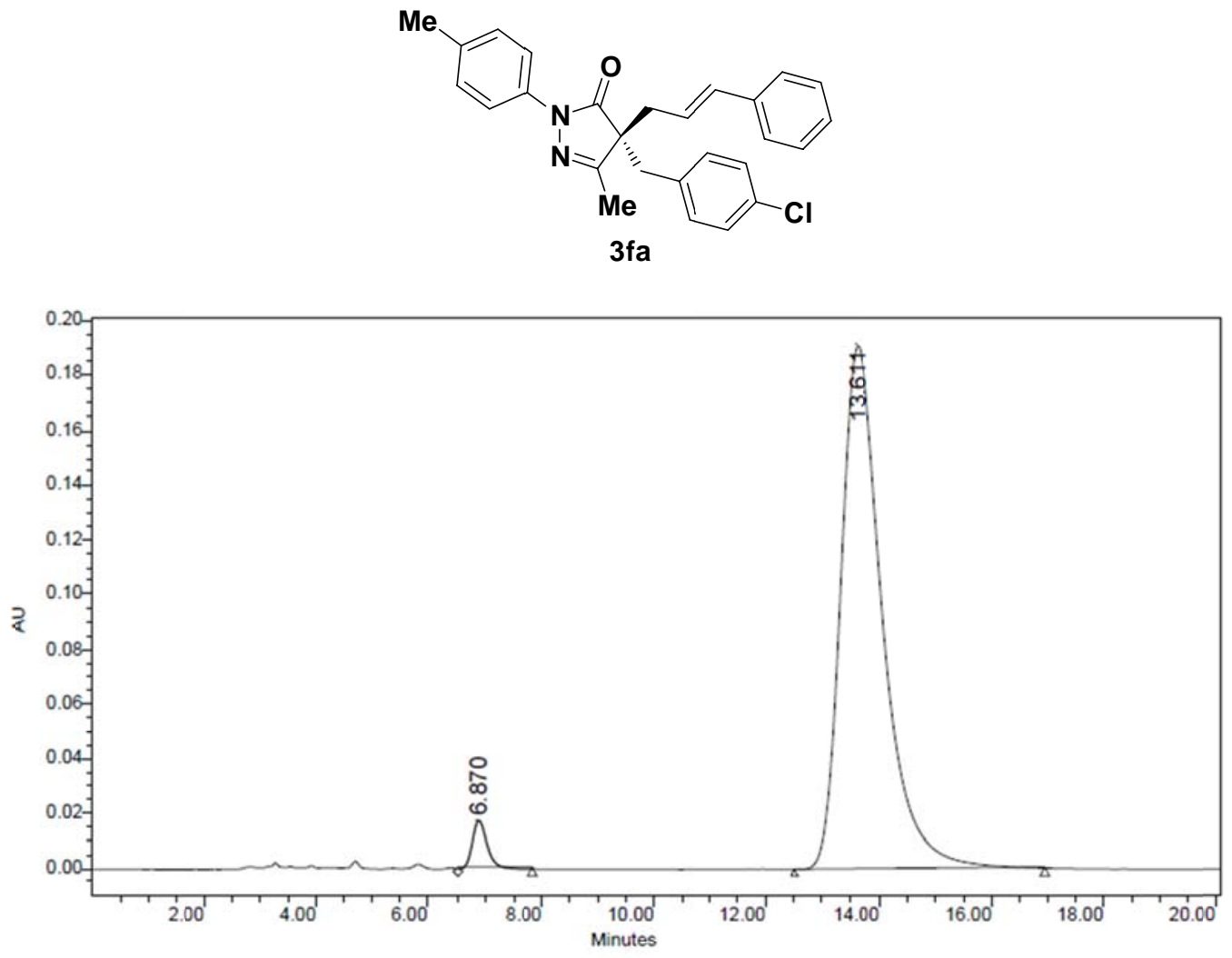

\begin{tabular}{|c|c|c|r|r|r|}
\hline & $\begin{array}{c}\text { RT } \\
(\mathrm{min})\end{array}$ & $\begin{array}{c}\text { Area } \\
(\text { V*sec })\end{array}$ & $\%$ Area & $\begin{array}{c}\text { Height } \\
(\text { V })\end{array}$ & $\begin{array}{c}\% \\
\text { Height }\end{array}$ \\
\hline 1 & 6.870 & 322018 & 3.15 & 17615 & 8.44 \\
\hline 2 & 13.611 & 9887760 & 96.85 & 191035 & 91.56 \\
\hline
\end{tabular}




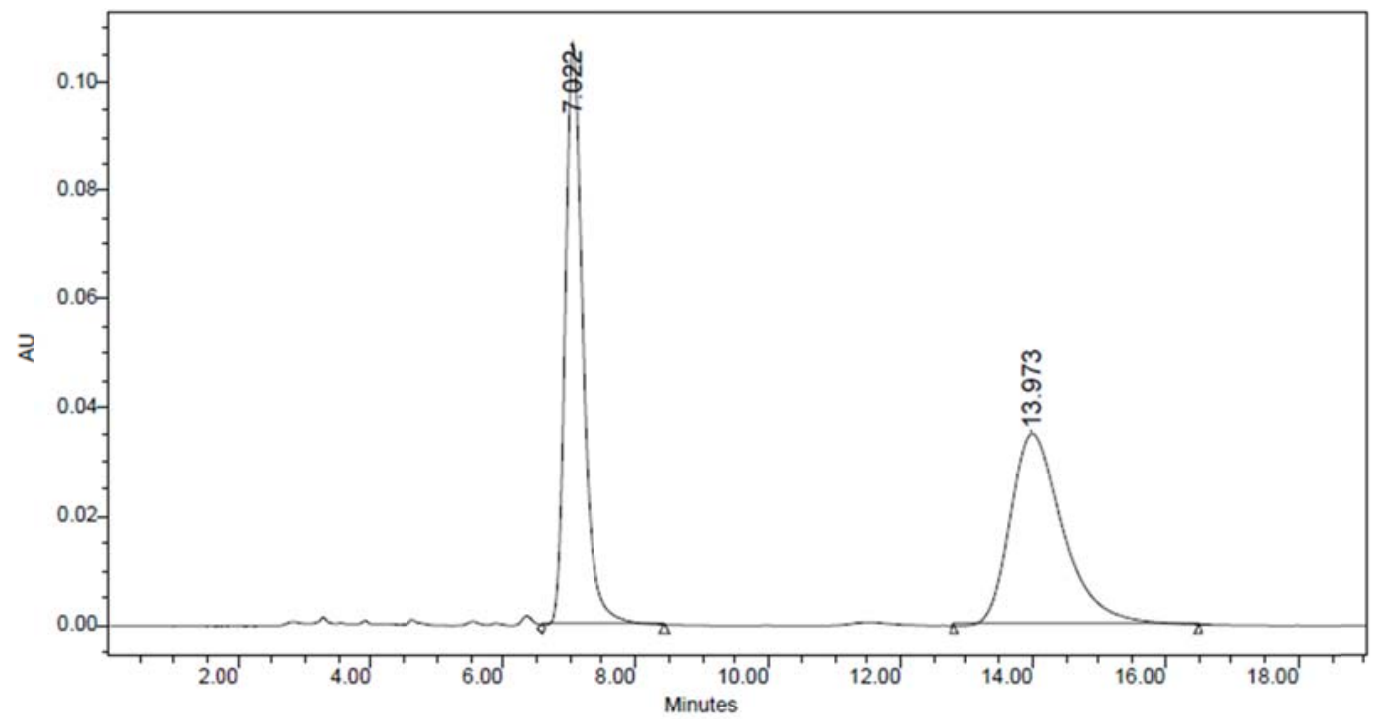

\begin{tabular}{|c|c|c|c|c|c|}
\hline & $\begin{array}{c}\text { RT } \\
(\mathrm{min})\end{array}$ & $\begin{array}{c}\text { Area } \\
\left(\mathrm{V}^{*} \mathrm{sec}\right)\end{array}$ & \% Area & $\begin{array}{c}\text { Height } \\
(\mathrm{V})\end{array}$ & $\begin{array}{c}\% \\
\text { Height }\end{array}$ \\
\hline 1 & 7.022 & 2011030 & 50.60 & 107296 & 75.20 \\
\hline 2 & 13.973 & 1963606 & 49.40 & 35379 & 24.80 \\
\hline
\end{tabular}
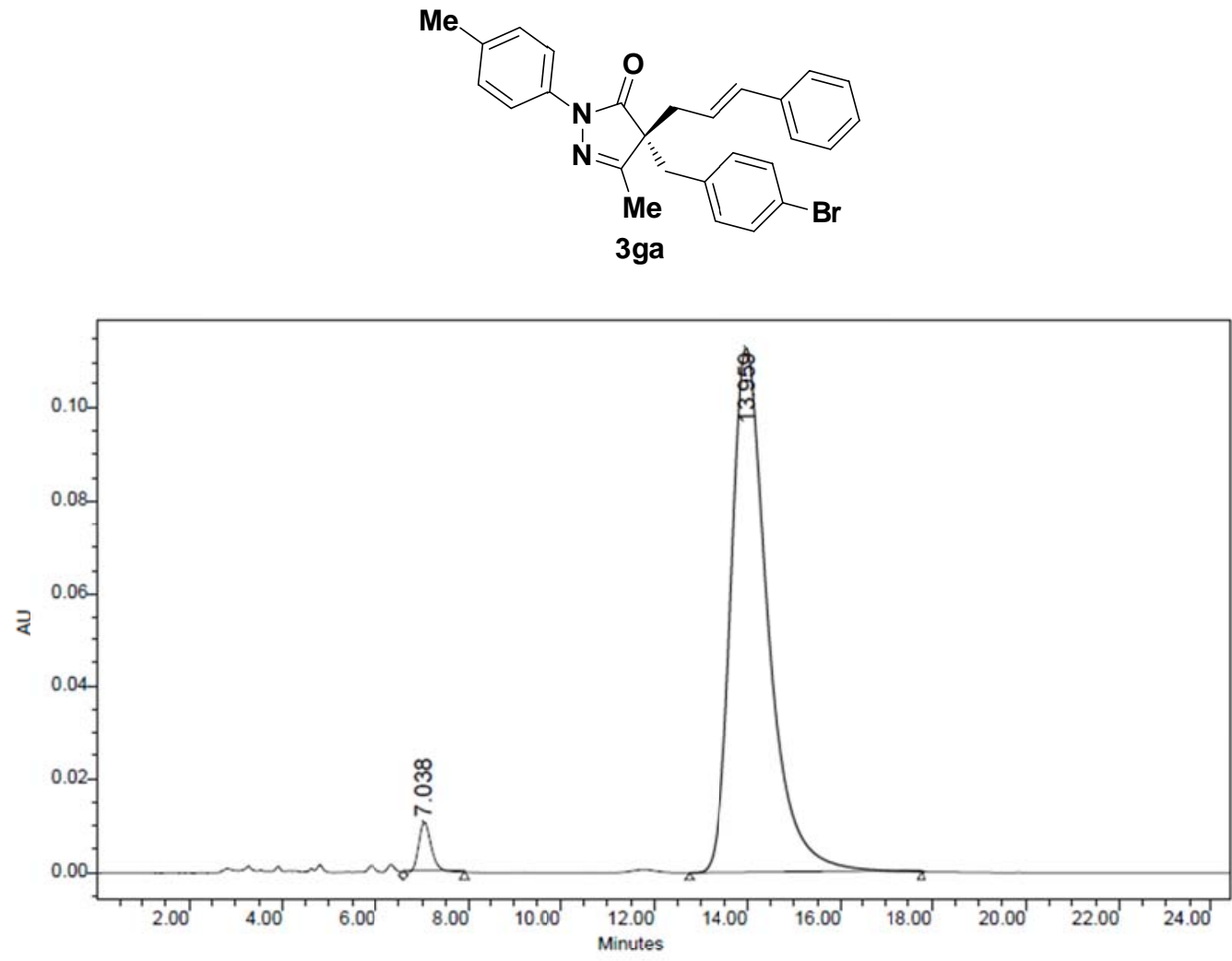

\begin{tabular}{|c|c|c|r|r|r|}
\hline & $\begin{array}{c}\text { RT } \\
(\mathrm{min})\end{array}$ & $\begin{array}{c}\text { Area } \\
\left(\mathrm{V}^{*} \mathrm{sec}\right)\end{array}$ & $\%$ Area & $\begin{array}{c}\text { Height } \\
(\mathrm{V})\end{array}$ & $\begin{array}{c}\% \\
\text { Height }\end{array}$ \\
\hline 1 & 7.038 & 205128 & 3.18 & 10755 & 8.69 \\
\hline 2 & 13.959 & 6236705 & 96.82 & 113066 & 91.31 \\
\hline
\end{tabular}




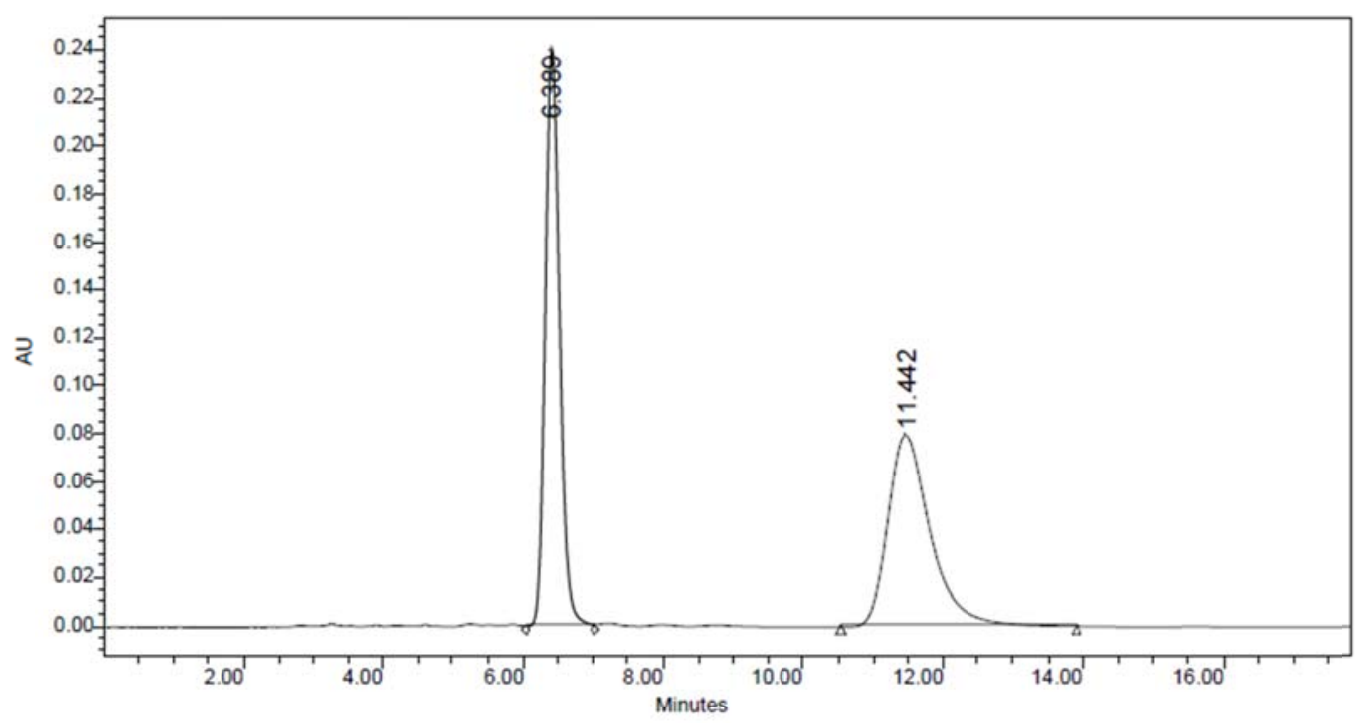

\begin{tabular}{|c|c|c|c|c|c|}
\hline & $\begin{array}{c}\text { RT } \\
(\mathrm{min})\end{array}$ & $\begin{array}{c}\text { Area } \\
\left(\mathrm{V}^{*} \mathrm{sec}\right)\end{array}$ & \% Area & $\begin{array}{c}\text { Height } \\
(\mathrm{V})\end{array}$ & $\begin{array}{c}\% \\
\text { Height }\end{array}$ \\
\hline 1 & 6.389 & 3443237 & 50.45 & 241043 & 75.14 \\
\hline 2 & 11.442 & 3381707 & 49.55 & 79769 & 24.86 \\
\hline
\end{tabular}
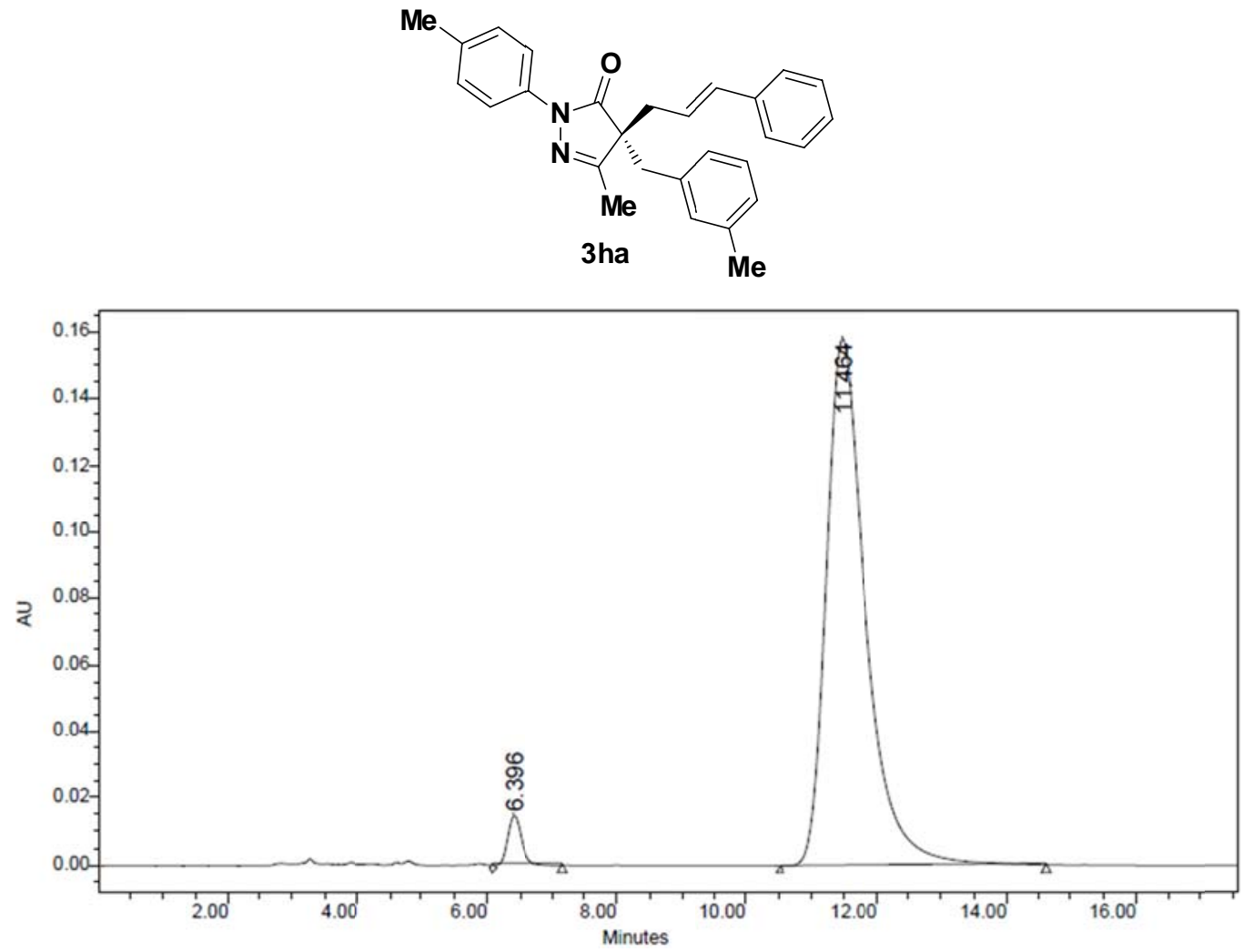

\begin{tabular}{|c|c|c|r|r|r|}
\hline & $\begin{array}{c}\text { RT } \\
(\mathrm{min})\end{array}$ & $\begin{array}{c}\text { Area } \\
\left(\mathrm{V}^{*} \mathrm{sec}\right)\end{array}$ & $\%$ Area & $\begin{array}{c}\text { Height } \\
(\mathrm{V})\end{array}$ & $\begin{array}{c}\% \\
\text { Height }\end{array}$ \\
\hline 1 & 6.396 & 223595 & 3.22 & 14922 & 8.62 \\
\hline 2 & 11.464 & 6713958 & 96.78 & 158277 & 91.38 \\
\hline
\end{tabular}




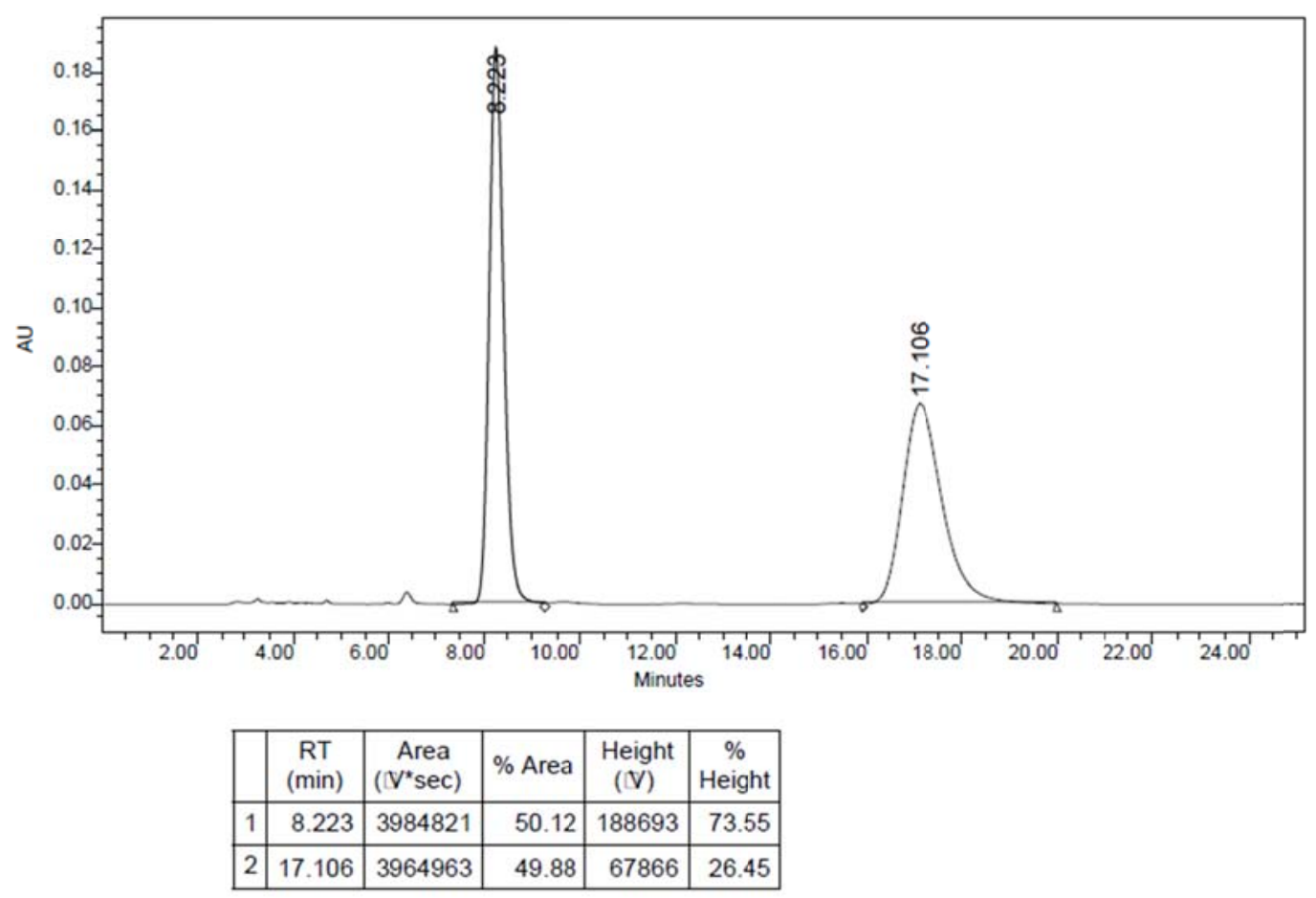

Me<smiles>COc1cccc(C[C@]2(C/C=C/c3ccccc3)C(=O)N(c3ccc(F)cc3)N=C2C)c1</smiles>

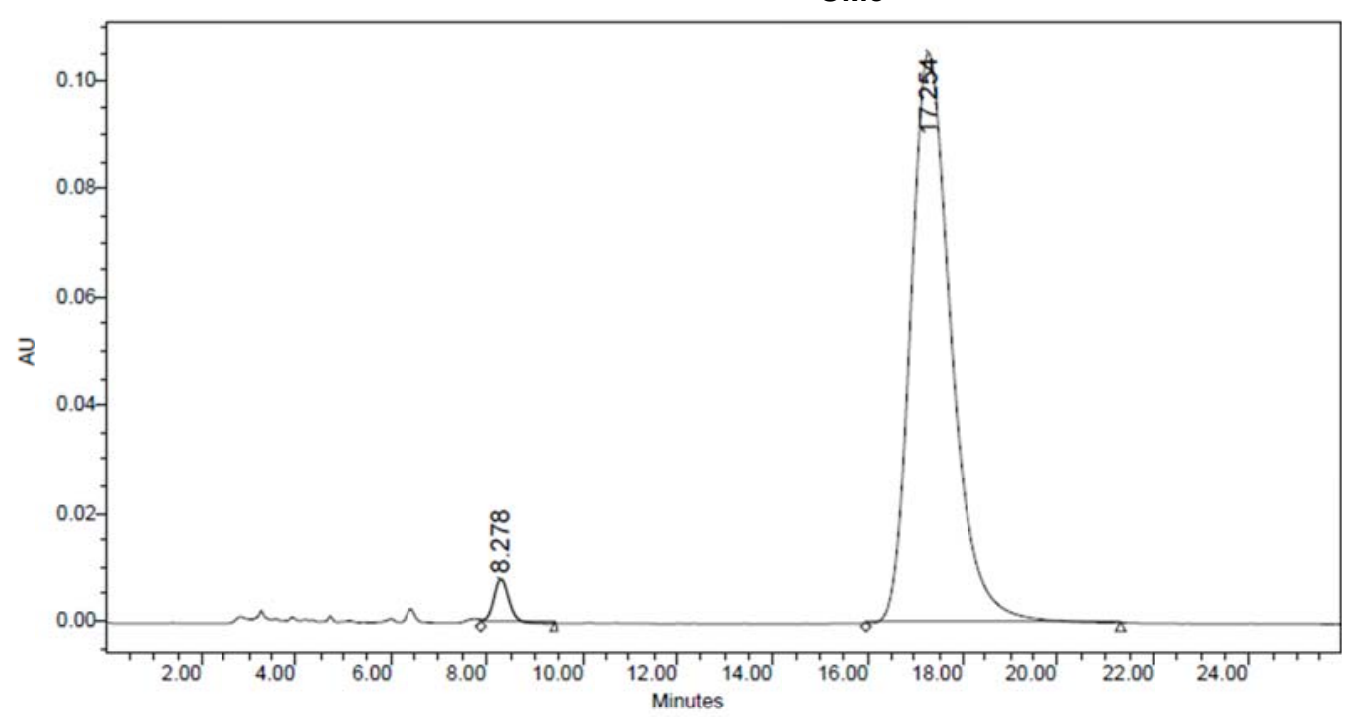

\begin{tabular}{|l|c|c|r|r|r|}
\hline & $\begin{array}{c}\mathrm{RT} \\
(\mathrm{min})\end{array}$ & $\begin{array}{c}\text { Area } \\
\left(\mathrm{V}^{*} \mathrm{sec}\right)\end{array}$ & $\%$ Area & $\begin{array}{c}\text { Height } \\
(\mathrm{V})\end{array}$ & $\begin{array}{c}\% \\
\text { Height }\end{array}$ \\
\hline 1 & 8.278 & 186131 & 2.88 & 8222 & 7.23 \\
\hline 2 & 17.254 & 6274775 & 97.12 & 105534 & 92.77 \\
\hline
\end{tabular}




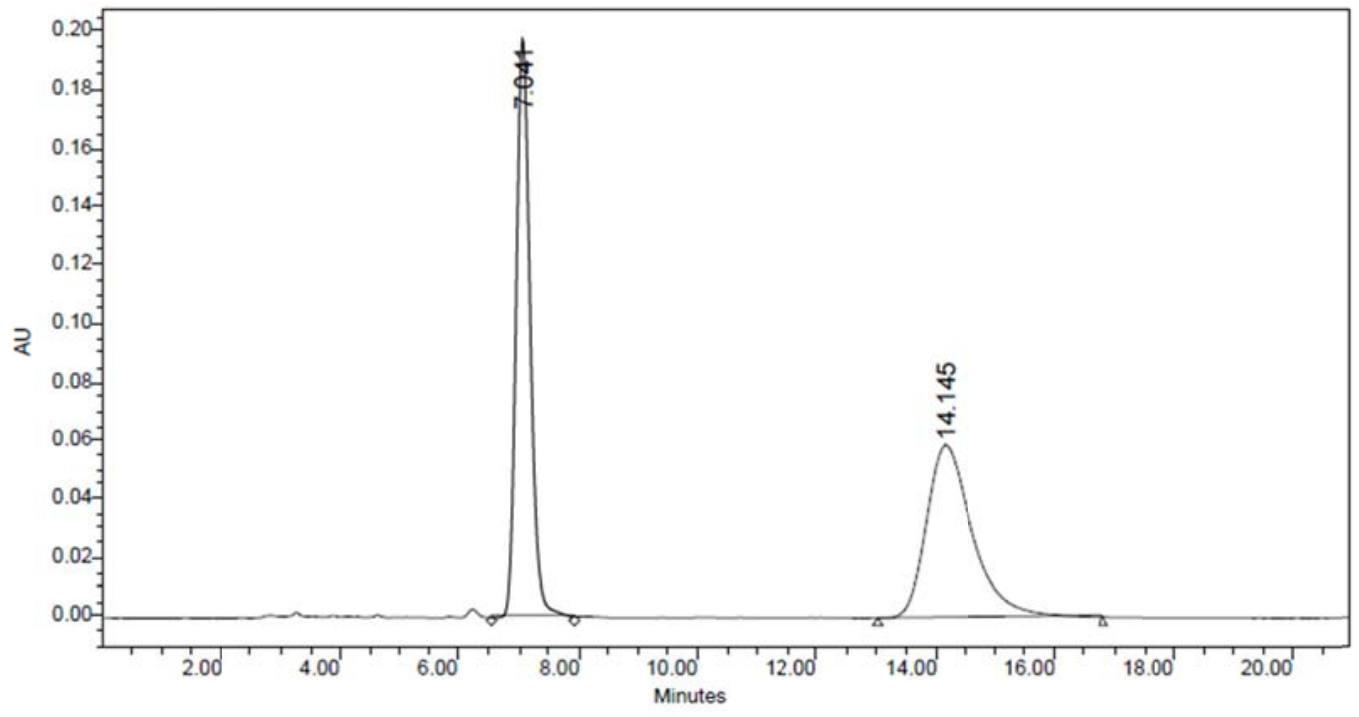

\begin{tabular}{|c|c|c|c|c|c|}
\hline & $\begin{array}{c}\text { RT } \\
(\mathrm{min})\end{array}$ & $\begin{array}{c}\text { Area } \\
\left(\mathrm{V}^{*} \mathrm{sec}\right)\end{array}$ & \% Area & $\begin{array}{c}\text { Height } \\
(\mathrm{V})\end{array}$ & $\begin{array}{c}\% \\
\text { Height }\end{array}$ \\
\hline 1 & 7.041 & 3227101 & 50.42 & 197787 & 77.08 \\
\hline 2 & 14.145 & 3173417 & 49.58 & 58809 & 22.92 \\
\hline
\end{tabular}

Me<smiles>CC1=NN(c2ccc(C)cc2)C(=O)[C@]1(C/C=C/c1ccccc1)Cc1cccc(Cl)c1</smiles>

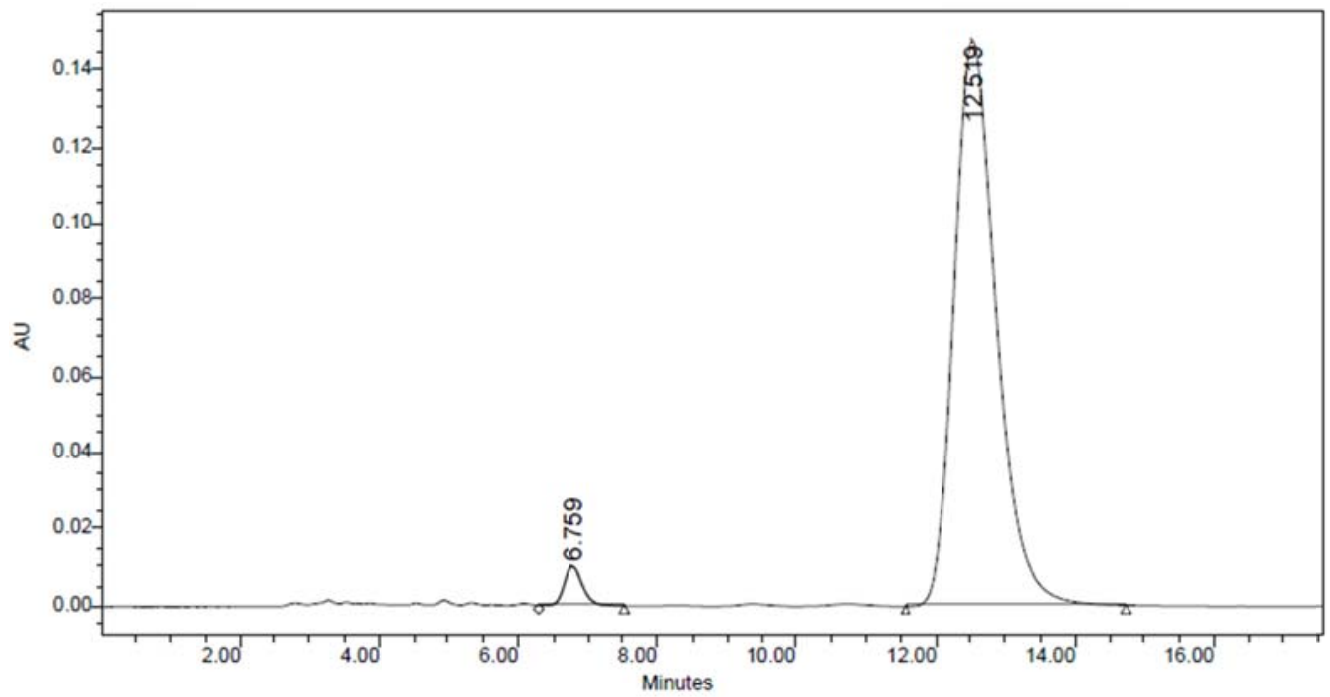

\begin{tabular}{|l|c|c|r|c|r|}
\hline & $\begin{array}{c}\text { RT } \\
(\mathrm{min})\end{array}$ & $\begin{array}{c}\text { Area } \\
\left(\mathrm{V}^{*} \mathrm{sec}\right)\end{array}$ & $\%$ Area & $\begin{array}{c}\text { Height } \\
(\mathrm{V})\end{array}$ & $\begin{array}{c}\% \\
\text { Height }\end{array}$ \\
\hline 1 & 6.759 & 191551 & 2.98 & 10261 & 6.50 \\
\hline 2 & 12.519 & 6246136 & 97.02 & 147722 & 93.50 \\
\hline
\end{tabular}




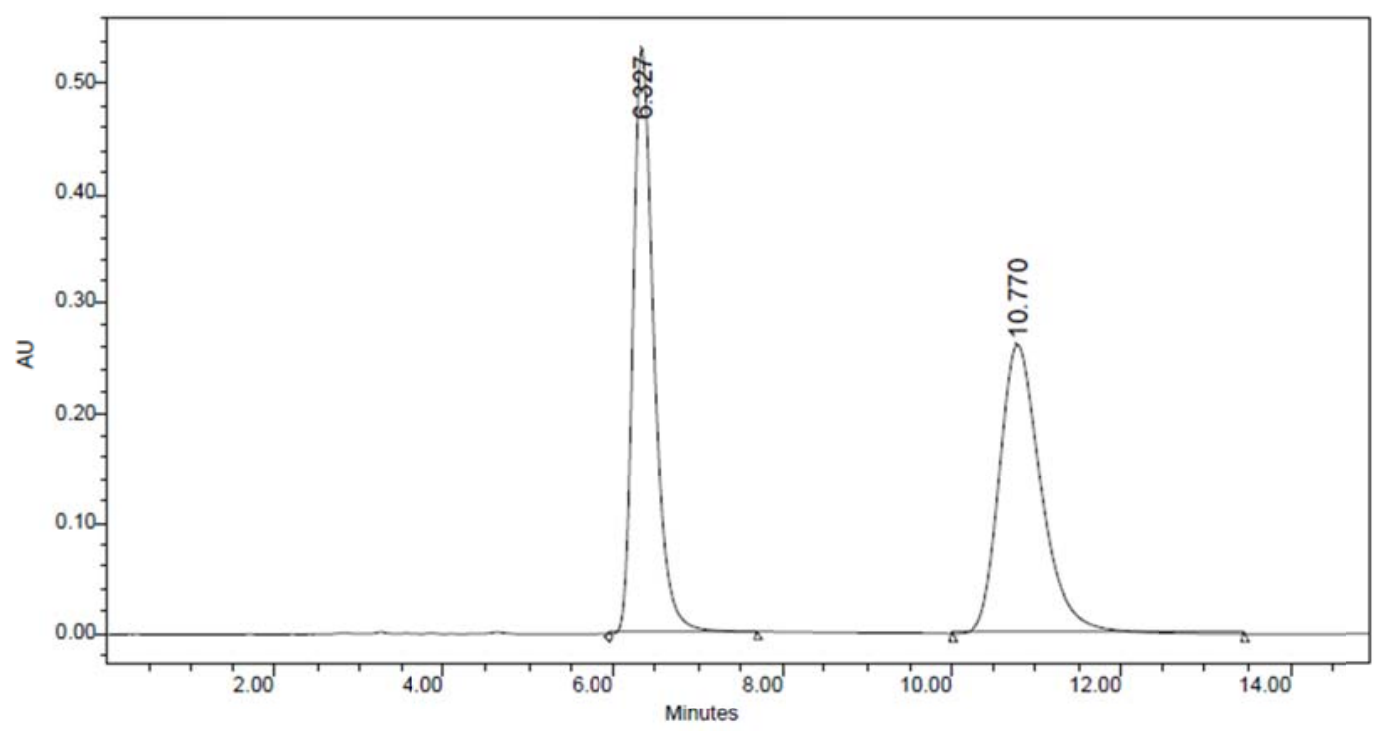

\begin{tabular}{|c|c|c|c|c|c|}
\hline & $\begin{array}{c}\text { RT } \\
(\mathrm{min})\end{array}$ & $\begin{array}{c}\text { Area } \\
\left(\mathrm{V}^{*} \mathrm{sec}\right)\end{array}$ & $\%$ Area & $\begin{array}{c}\text { Height } \\
(\mathrm{V})\end{array}$ & $\begin{array}{c}\% \\
\text { Height }\end{array}$ \\
\hline 1 & 6.327 & 9067109 & 50.13 & 533629 & 67.01 \\
\hline 2 & 10.770 & 9020890 & 49.87 & 262722 & 32.99 \\
\hline
\end{tabular}

Me

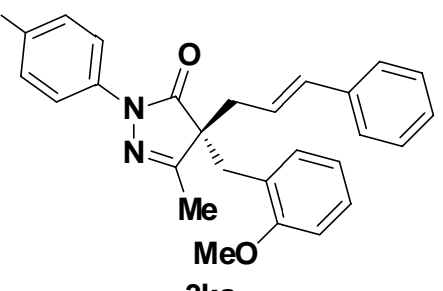

3ka

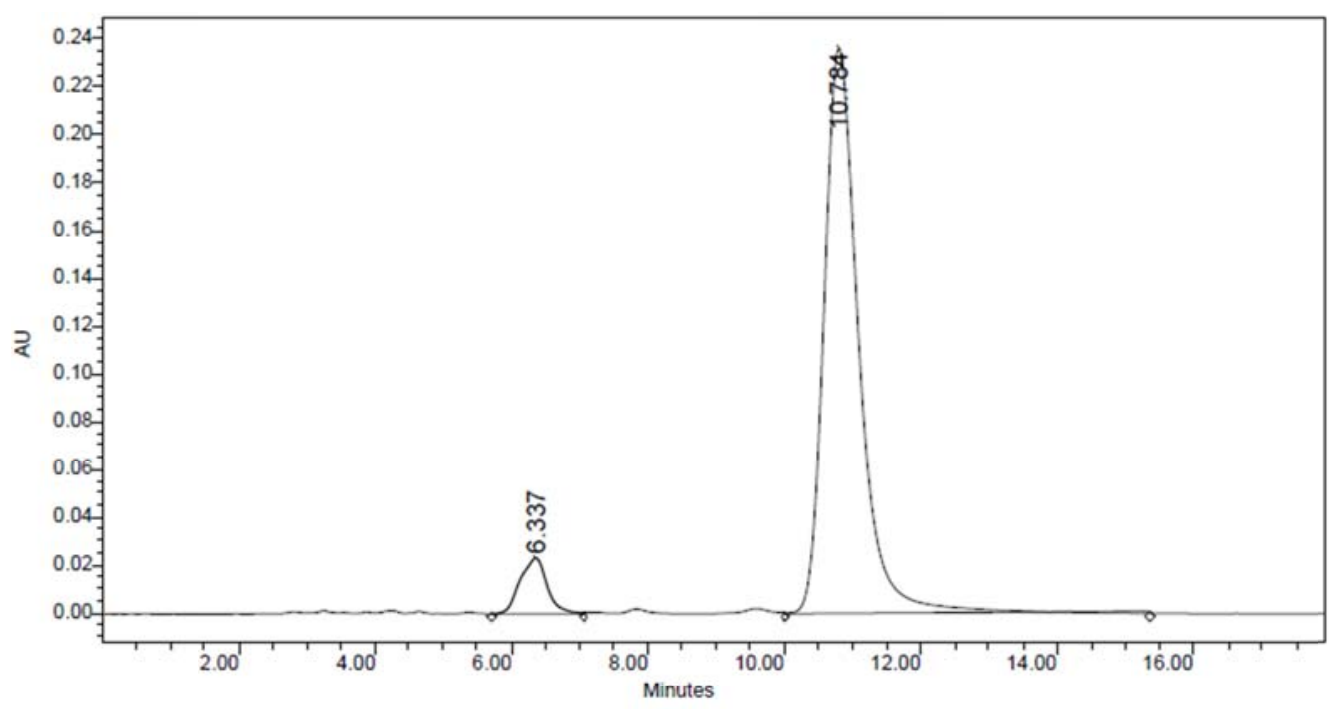

\begin{tabular}{|c|c|c|r|r|r|}
\hline & $\begin{array}{c}\text { RT } \\
(\mathrm{min})\end{array}$ & $\begin{array}{c}\text { Area } \\
\left(\mathrm{V}^{*} \mathrm{sec}\right)\end{array}$ & \% Area & $\begin{array}{l}\text { Height } \\
(\mathrm{V})\end{array}$ & $\begin{array}{c}\% \\
\text { Height }\end{array}$ \\
\hline 1 & 6.337 & 640376 & 7.04 & 23332 & 8.97 \\
\hline 2 & 10.784 & 8461290 & 92.96 & 236763 & 91.03 \\
\hline
\end{tabular}




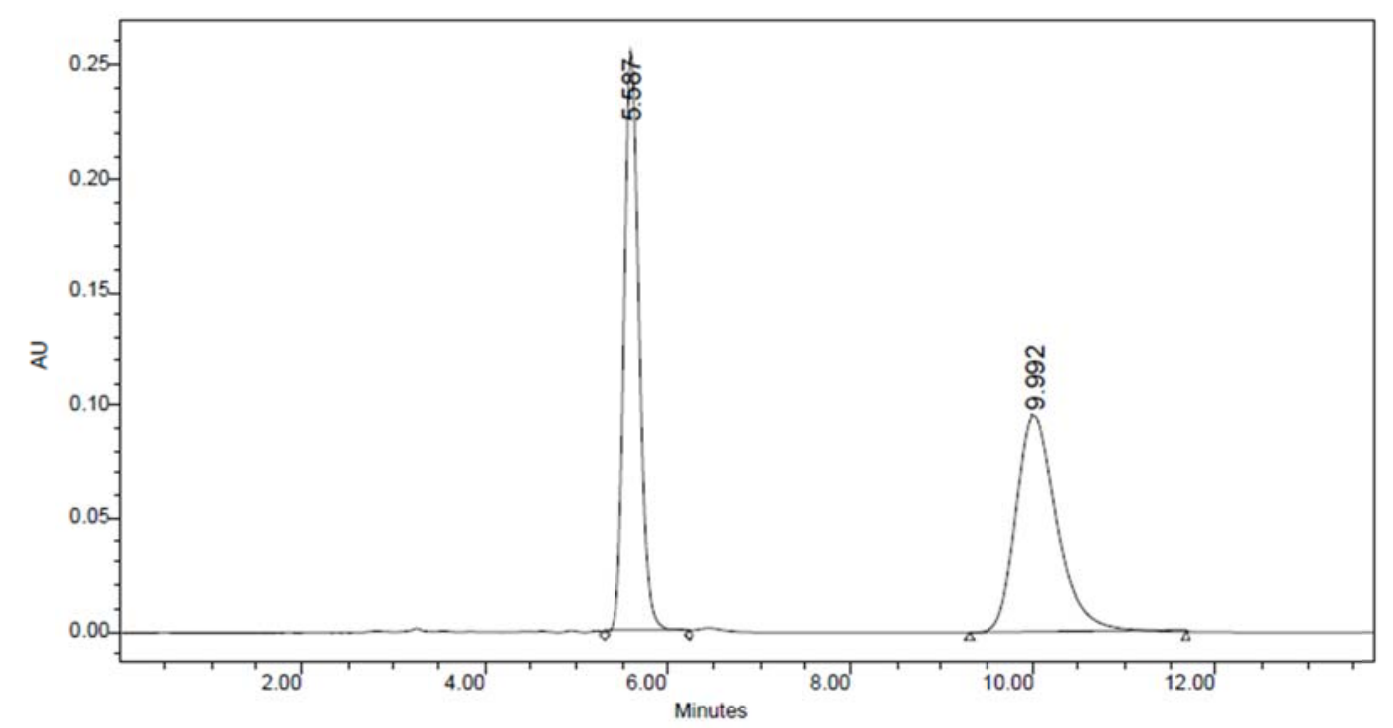

\begin{tabular}{|c|c|c|r|r|r|}
\hline & $\begin{array}{c}\text { RT } \\
(\mathrm{min})\end{array}$ & $\begin{array}{c}\text { Area } \\
(\mathrm{V} \text { (sec })\end{array}$ & $\%$ Area & $\begin{array}{c}\text { Height } \\
(\mathrm{V})\end{array}$ & $\begin{array}{c}\% \\
\text { Height }\end{array}$ \\
\hline 1 & 5.587 & 2966868 & 49.79 & 256645 & 72.76 \\
\hline 2 & 9.992 & 2992023 & 50.21 & 96071 & 27.24 \\
\hline
\end{tabular}

Me

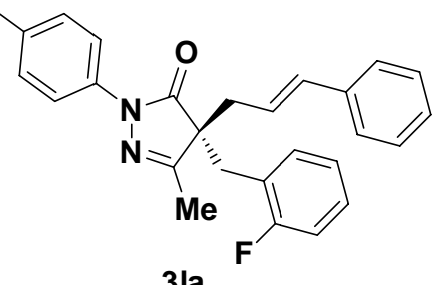

$31 a$

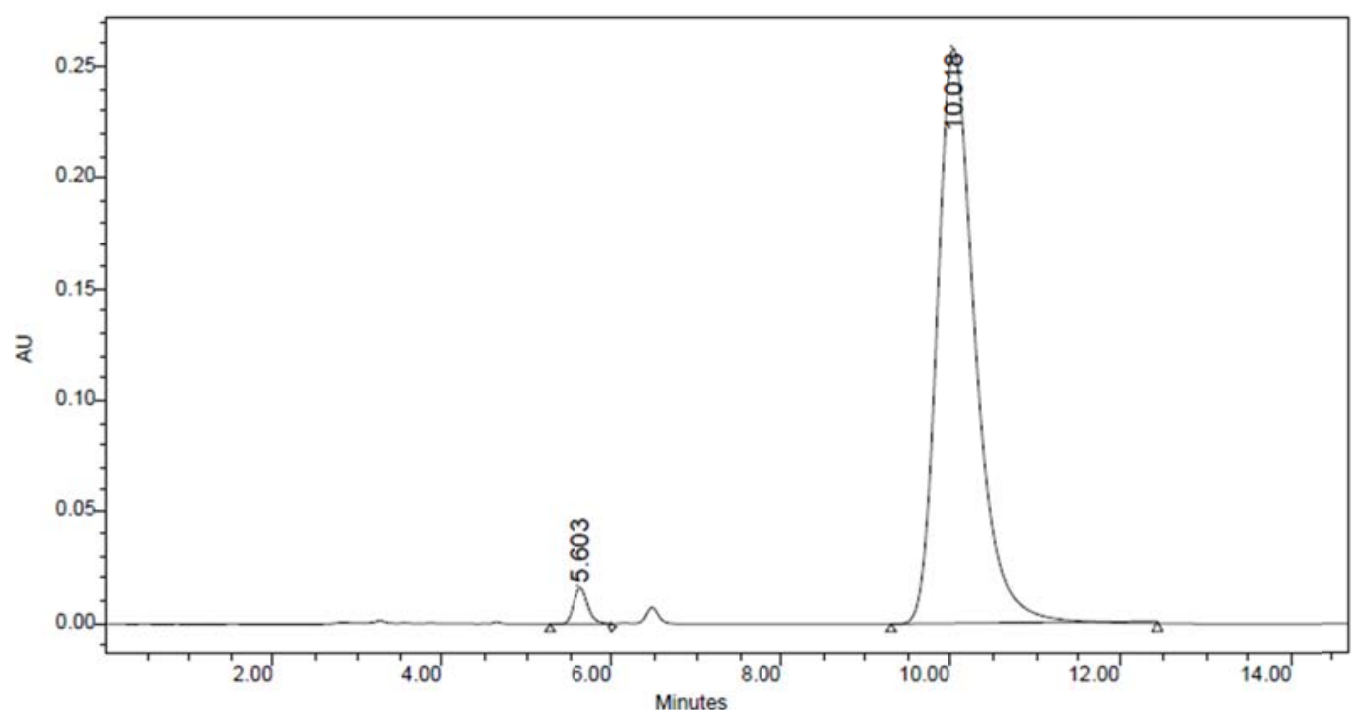

\begin{tabular}{|c|c|r|r|r|r|}
\hline & $\begin{array}{c}\text { RT } \\
(\mathrm{min})\end{array}$ & $\begin{array}{c}\text { Area } \\
\left(\mathrm{V}^{*} \mathrm{sec}\right)\end{array}$ & \% Area & $\begin{array}{c}\text { Height } \\
(\mathrm{V})\end{array}$ & $\begin{array}{c}\% \\
\text { Height }\end{array}$ \\
\hline 1 & 5.603 & 191823 & 2.31 & 16329 & 5.94 \\
\hline 2 & 10.018 & 8118120 & 97.69 & 258759 & 94.06 \\
\hline
\end{tabular}




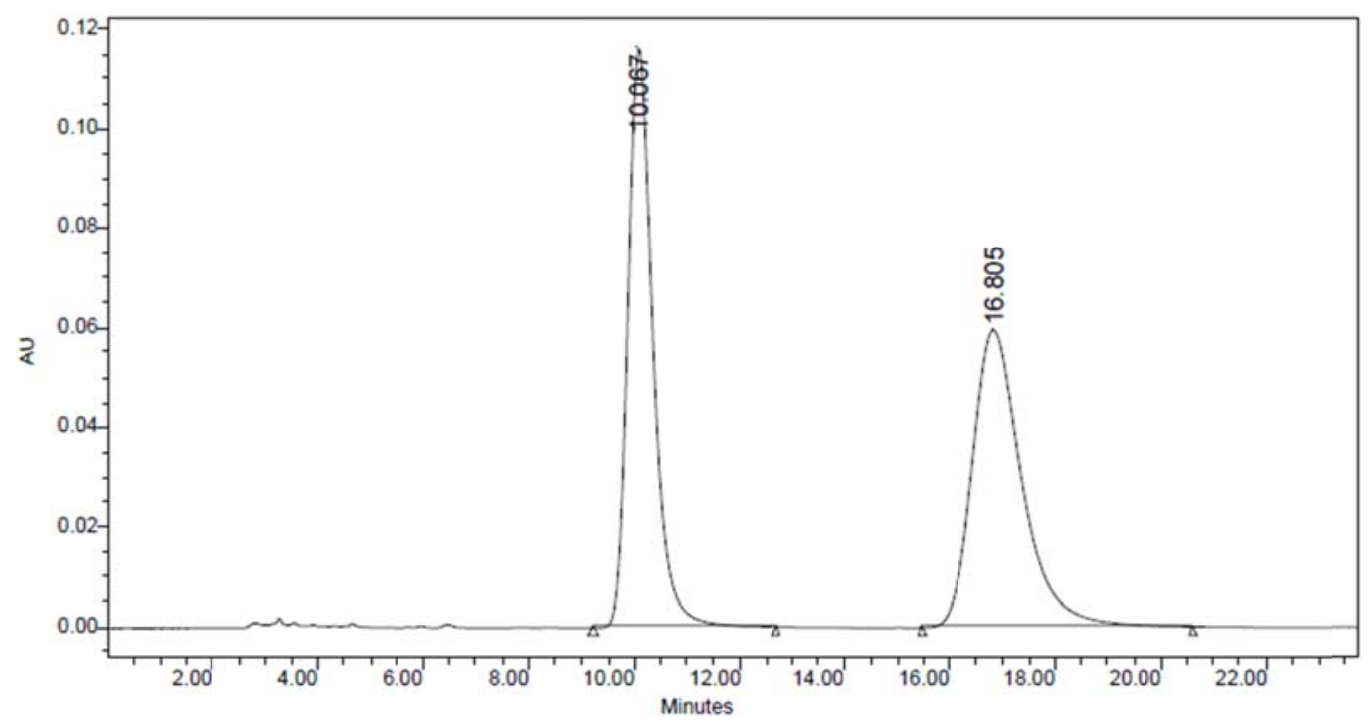

\begin{tabular}{|c|c|c|r|r|r|}
\hline & $\begin{array}{c}\text { RT } \\
(\mathrm{min})\end{array}$ & $\begin{array}{c}\text { Area } \\
\left(\mathrm{V}^{*} \mathrm{sec}\right)\end{array}$ & \% Area & $\begin{array}{c}\text { Height } \\
(\mathrm{V})\end{array}$ & $\begin{array}{c}\% \\
\text { Height }\end{array}$ \\
\hline 1 & 10.067 & 3817703 & 49.48 & 116082 & 66.10 \\
\hline 2 & 16.805 & 3897396 & 50.52 & 59546 & 33.90 \\
\hline
\end{tabular}

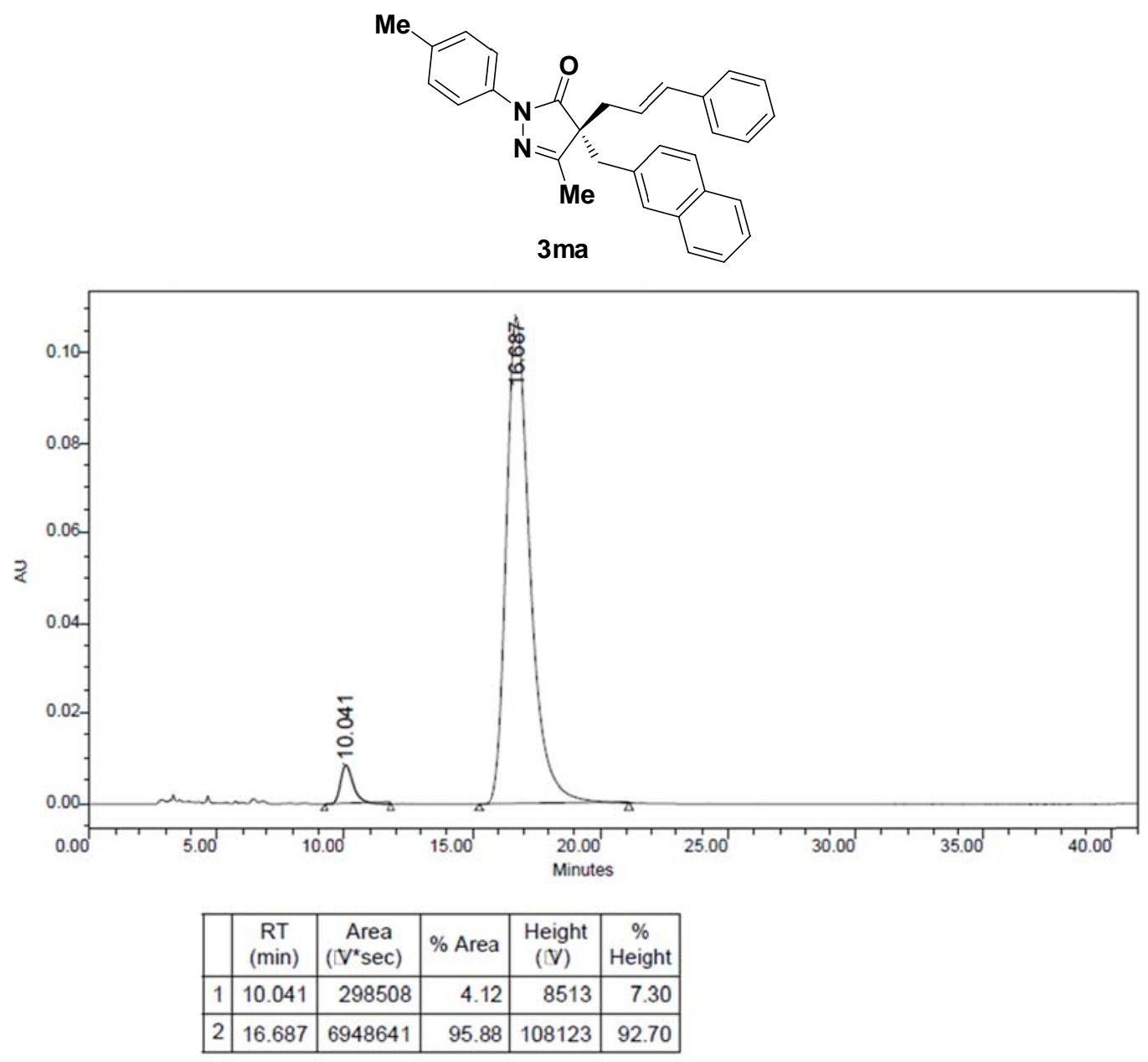




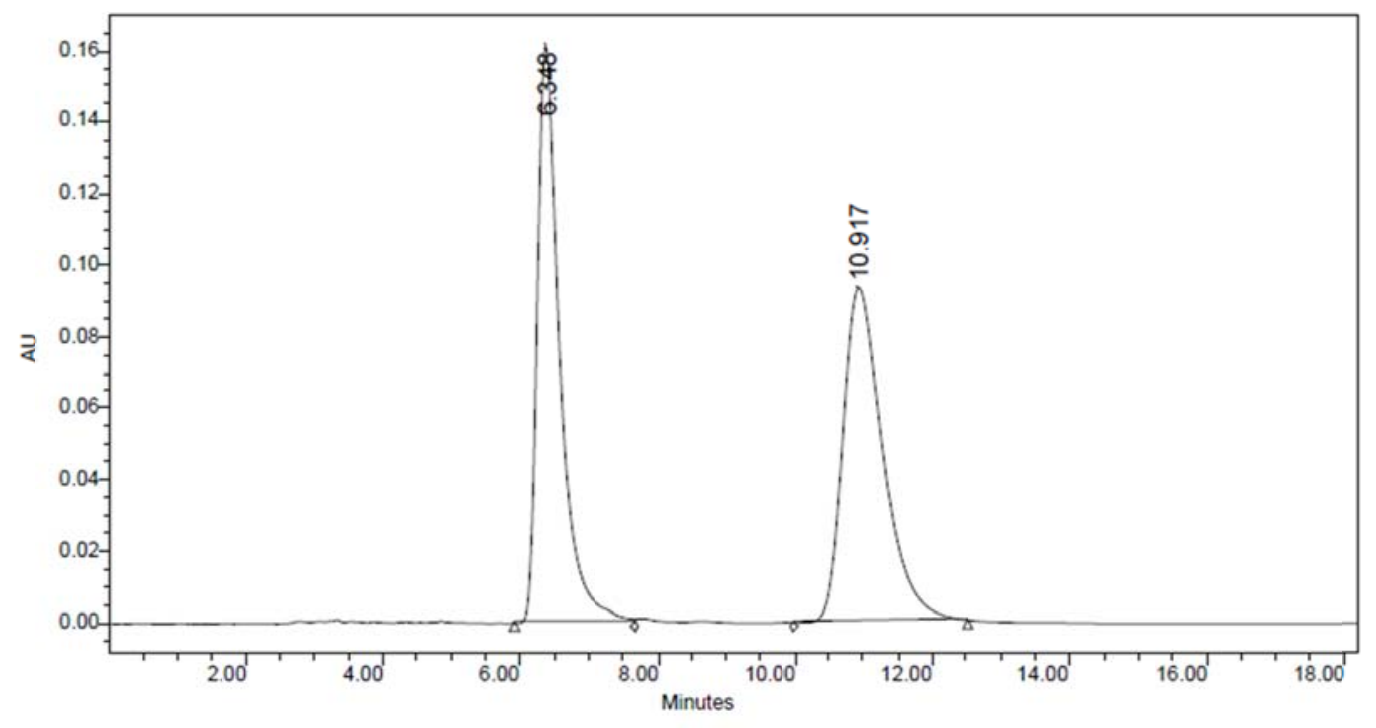

\begin{tabular}{|c|c|c|c|c|c|}
\hline & $\begin{array}{c}\text { RT } \\
(\mathrm{min})\end{array}$ & $\begin{array}{c}\text { Area } \\
\left(\mathrm{V}^{*} \mathrm{sec}\right)\end{array}$ & \% Area & $\begin{array}{c}\text { Height } \\
(\mathrm{V})\end{array}$ & $\begin{array}{c}\% \\
\text { Height }\end{array}$ \\
\hline 1 & 6.348 & 3716495 & 49.57 & 161538 & 63.37 \\
\hline 2 & 10.917 & 3781331 & 50.43 & 93392 & 36.63 \\
\hline
\end{tabular}

Me

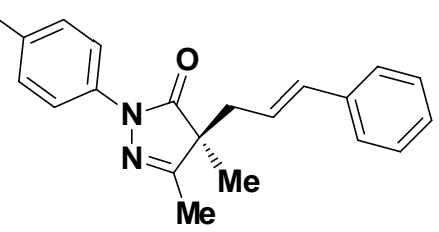

3na

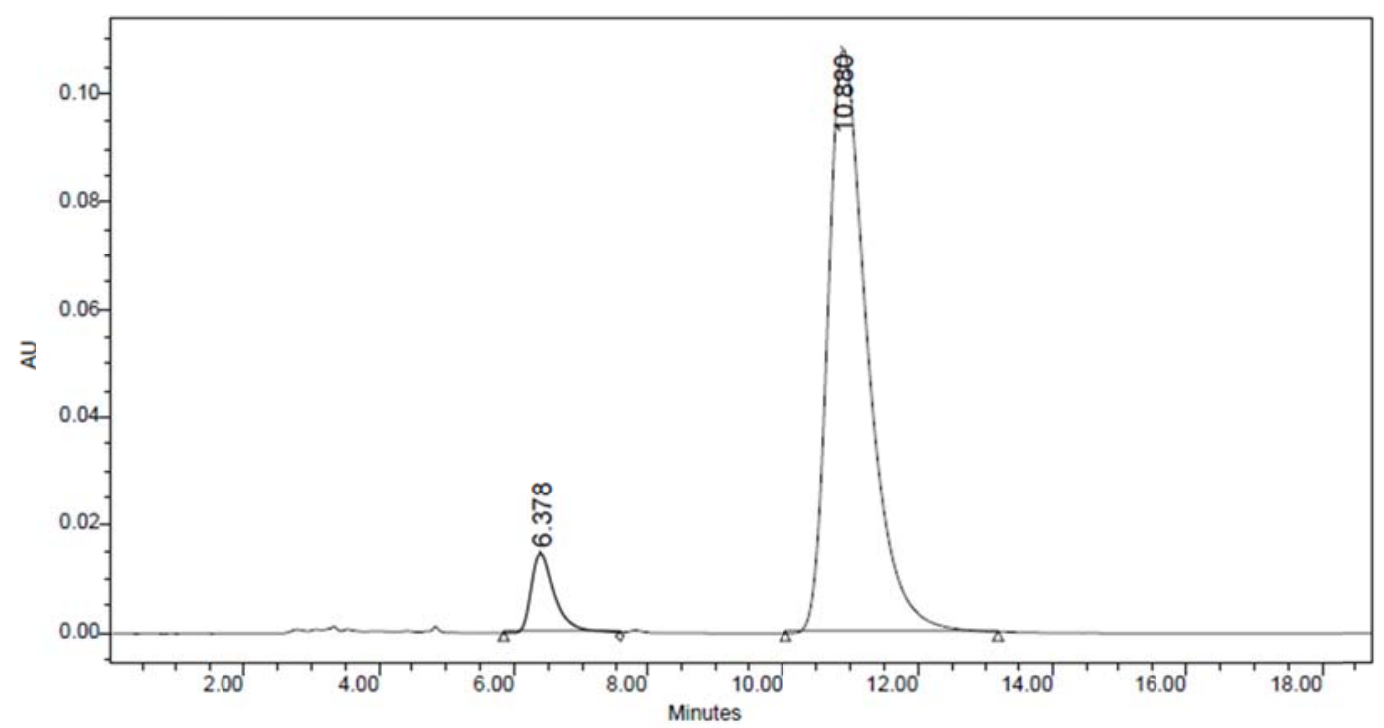

\begin{tabular}{|c|c|c|r|c|c|}
\hline & $\begin{array}{c}\text { RT } \\
(\mathrm{min})\end{array}$ & $\begin{array}{c}\text { Area } \\
\left(\mathrm{V}^{*} \mathrm{sec}\right)\end{array}$ & \% Area & $\begin{array}{c}\text { Height } \\
(\mathrm{V})\end{array}$ & $\begin{array}{c}\% \\
\text { Height }\end{array}$ \\
\hline 1 & 6.378 & 361995 & 7.57 & 14724 & 11.95 \\
\hline 2 & 10.880 & 4417533 & 92.43 & 108515 & 88.05 \\
\hline
\end{tabular}




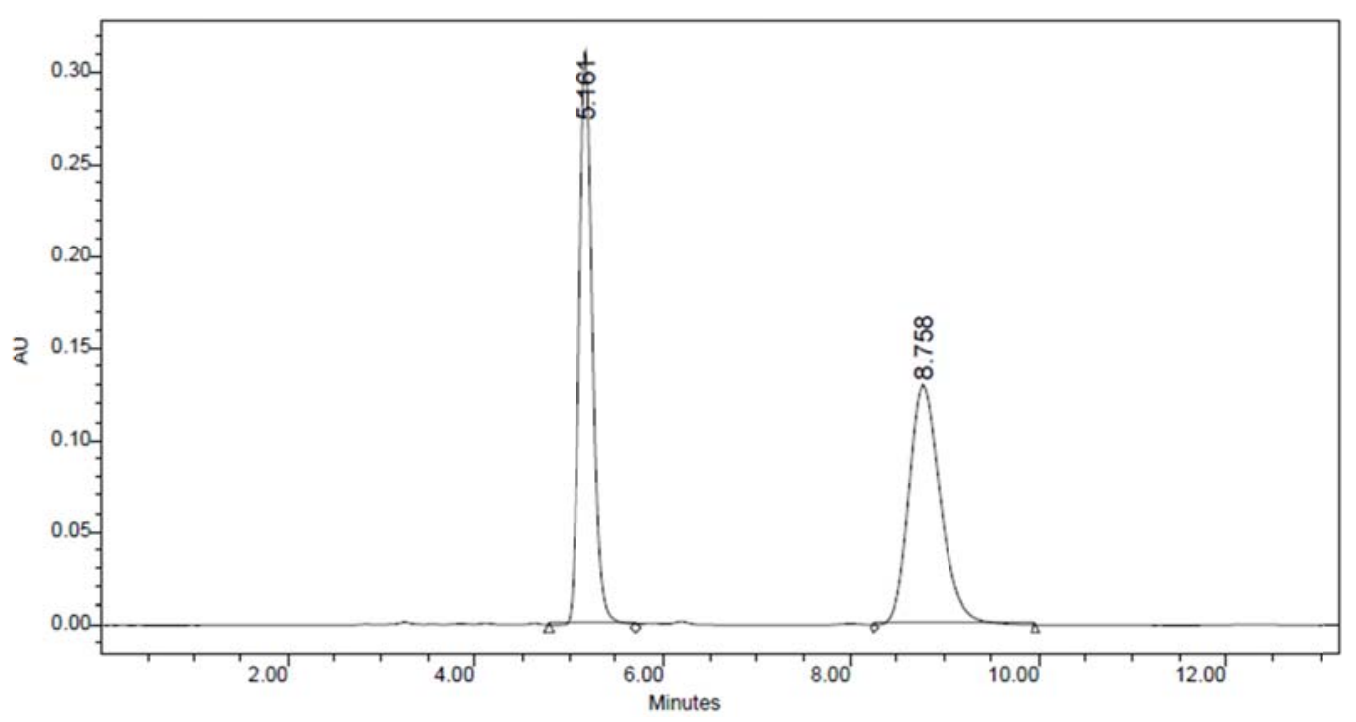

\begin{tabular}{|c|c|c|c|c|c|}
\hline & $\begin{array}{c}\text { RT } \\
(\mathrm{min})\end{array}$ & $\begin{array}{c}\text { Area } \\
\left(\mathrm{V}^{*} \mathrm{sec}\right)\end{array}$ & \% Area & $\begin{array}{c}\text { Height } \\
(\mathrm{V})\end{array}$ & $\begin{array}{c}\% \\
\text { Height }\end{array}$ \\
\hline 1 & 5.161 & 3099525 & 50.03 & 313023 & 70.58 \\
\hline 2 & 8.758 & 3095409 & 49.97 & 130450 & 29.42 \\
\hline
\end{tabular}

Me

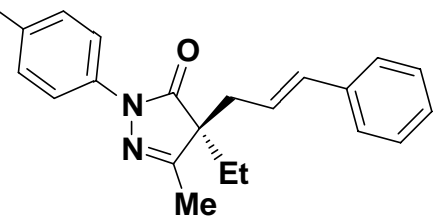

$30 a$

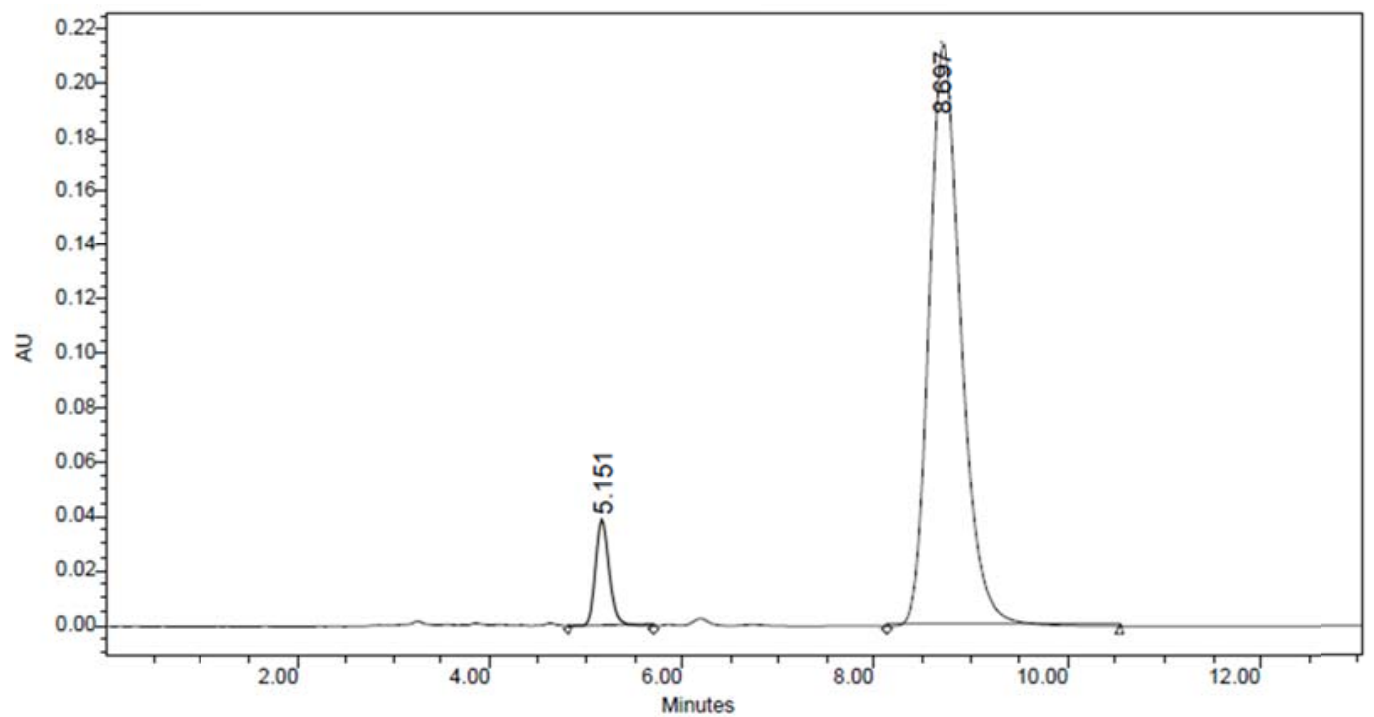

\begin{tabular}{|c|c|c|r|r|r|}
\hline & $\begin{array}{c}\text { RT } \\
(\mathrm{min})\end{array}$ & $\begin{array}{c}\text { Area } \\
\left(\mathrm{V}^{*} \mathrm{sec}\right)\end{array}$ & $\%$ Area & $\begin{array}{c}\text { Height } \\
(\mathrm{V})\end{array}$ & $\begin{array}{c}\% \\
\text { Height }\end{array}$ \\
\hline 1 & 5.151 & 392666 & 7.24 & 38928 & 15.35 \\
\hline 2 & 8.697 & 5034357 & 92.76 & 214603 & 84.65 \\
\hline
\end{tabular}




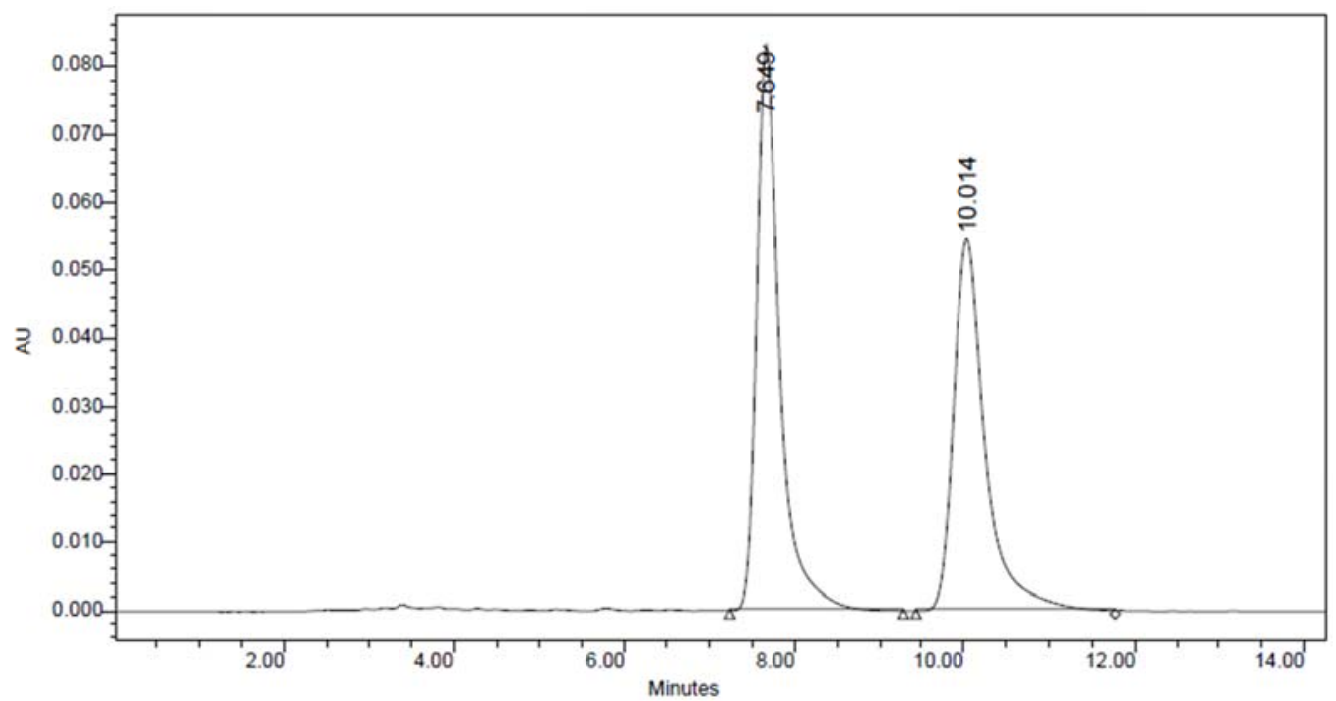

\begin{tabular}{|c|c|c|c|c|c|}
\hline & $\begin{array}{c}\mathrm{RT} \\
(\mathrm{min})\end{array}$ & $\begin{array}{c}\text { Area } \\
\left(\mathrm{V}^{*} \mathrm{sec}\right)\end{array}$ & $\%$ Area & $\begin{array}{c}\text { Height } \\
(\mathrm{V})\end{array}$ & $\begin{array}{c}\% \\
\text { Height }\end{array}$ \\
\hline 1 & 7.649 & 1586947 & 52.77 & 83233 & 60.25 \\
\hline 2 & 10.014 & 1420375 & 47.23 & 54905 & 39.75 \\
\hline
\end{tabular}
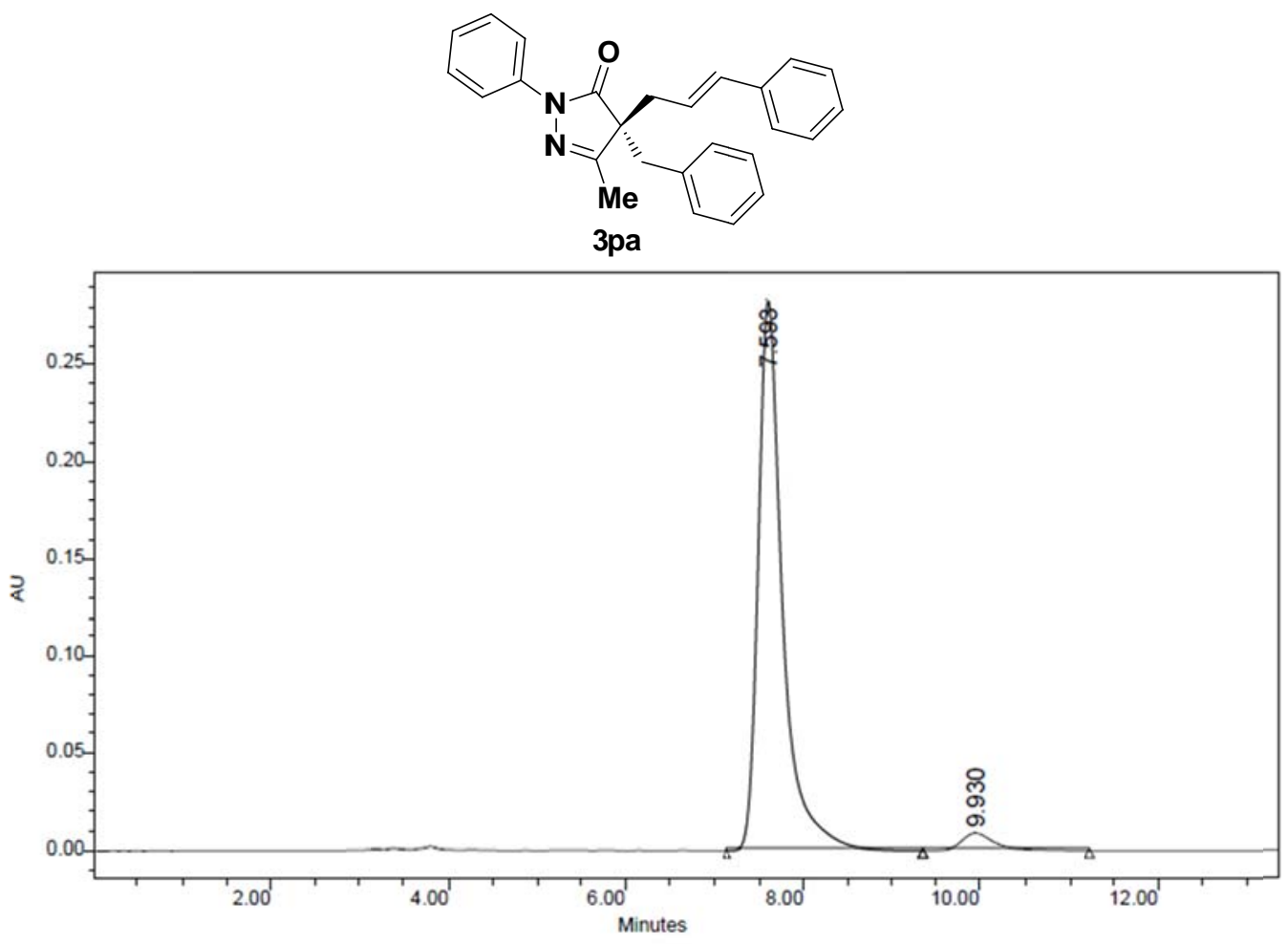

\begin{tabular}{|l|c|c|r|r|r|}
\hline & $\begin{array}{c}\text { RT } \\
(\mathrm{min})\end{array}$ & $\begin{array}{c}\text { Area } \\
\left(\mathrm{V}^{*} \mathrm{sec}\right)\end{array}$ & \% Area & $\begin{array}{c}\text { Height } \\
(\mathrm{V})\end{array}$ & $\begin{array}{c}\% \\
\text { Height }\end{array}$ \\
\hline 1 & 7.593 & 5395090 & 96.01 & 283403 & 96.97 \\
\hline 2 & 9.930 & 224087 & 3.99 & 8850 & 3.03 \\
\hline
\end{tabular}




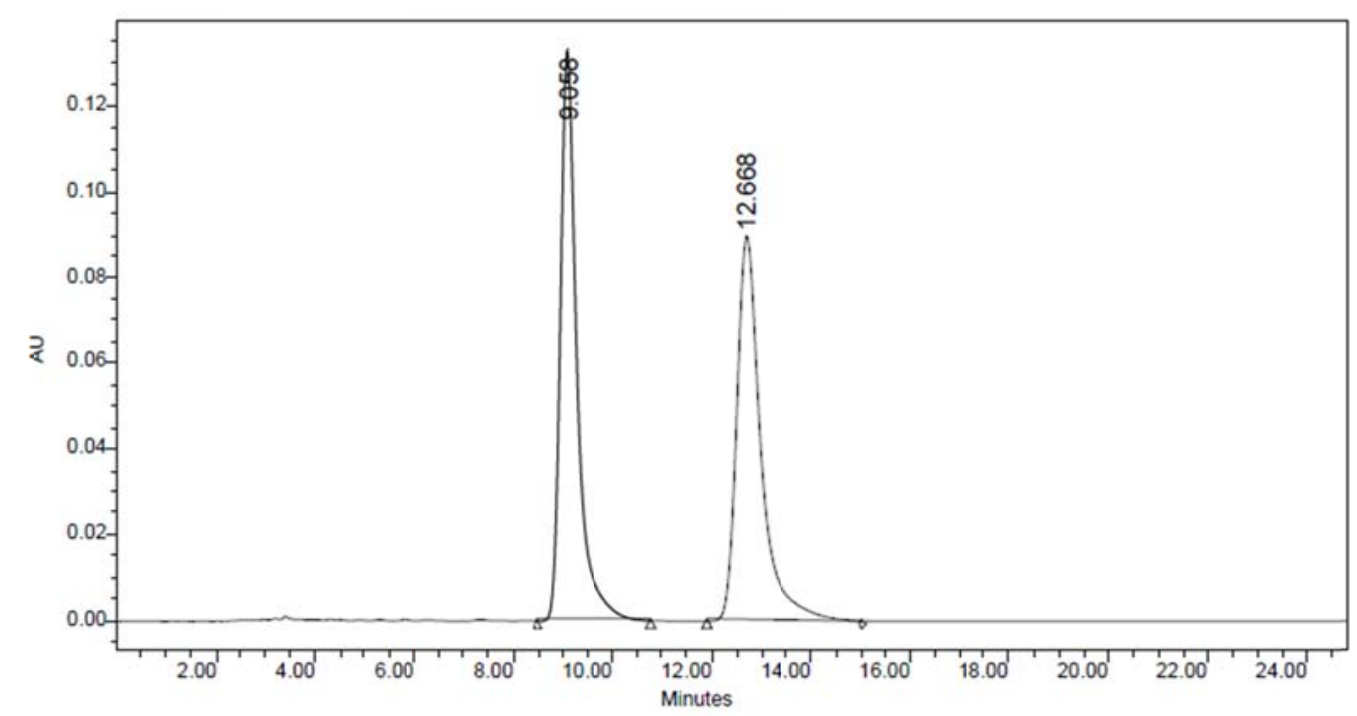

\begin{tabular}{|c|c|c|c|c|c|}
\hline & $\begin{array}{c}\text { RT } \\
(\mathrm{min})\end{array}$ & $\begin{array}{c}\text { Area } \\
\left(\mathrm{V}^{*} \mathrm{sec}\right)\end{array}$ & $\%$ Area & $\begin{array}{c}\text { Height } \\
(\mathrm{V})\end{array}$ & $\begin{array}{c}\% \\
\text { Height }\end{array}$ \\
\hline 1 & 9.058 & 3077183 & 50.31 & 133038 & 59.67 \\
\hline 2 & 12.668 & 3039059 & 49.69 & 89927 & 40.33 \\
\hline
\end{tabular}

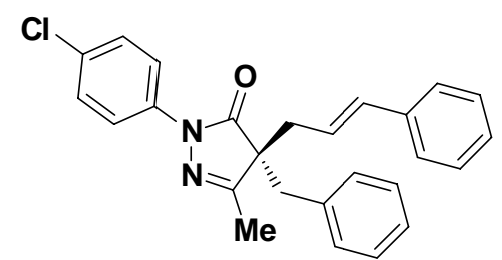

$3 q a$

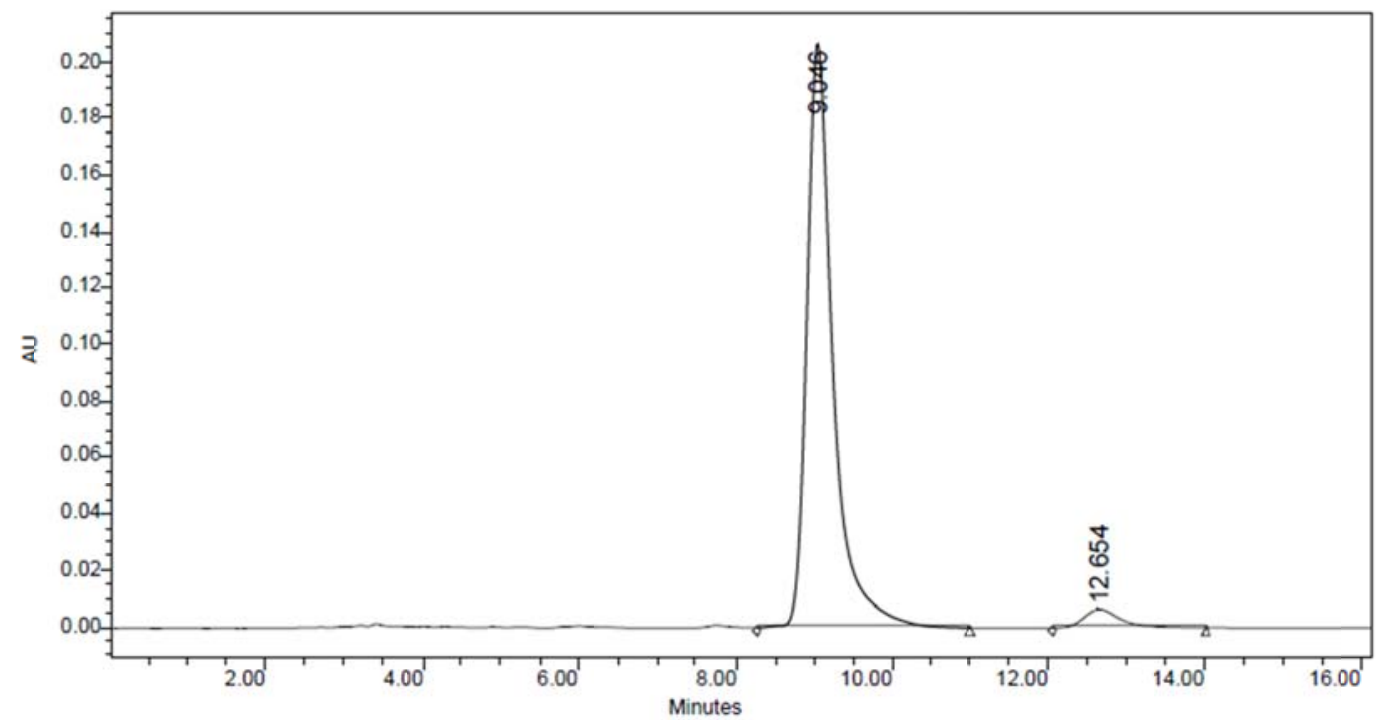

\begin{tabular}{|c|c|c|r|r|r|}
\hline & $\begin{array}{c}\text { RT } \\
(\mathrm{min})\end{array}$ & $\begin{array}{c}\text { Area } \\
\left(\mathrm{V}^{*} \mathrm{sec}\right)\end{array}$ & $\%$ Area & $\begin{array}{c}\text { Height } \\
(\mathrm{V})\end{array}$ & $\begin{array}{c}\% \\
\text { Height }\end{array}$ \\
\hline 1 & 9.046 & 4809324 & 95.83 & 206665 & 96.95 \\
\hline 2 & 12.654 & 209161 & 4.17 & 6502 & 3.05 \\
\hline
\end{tabular}



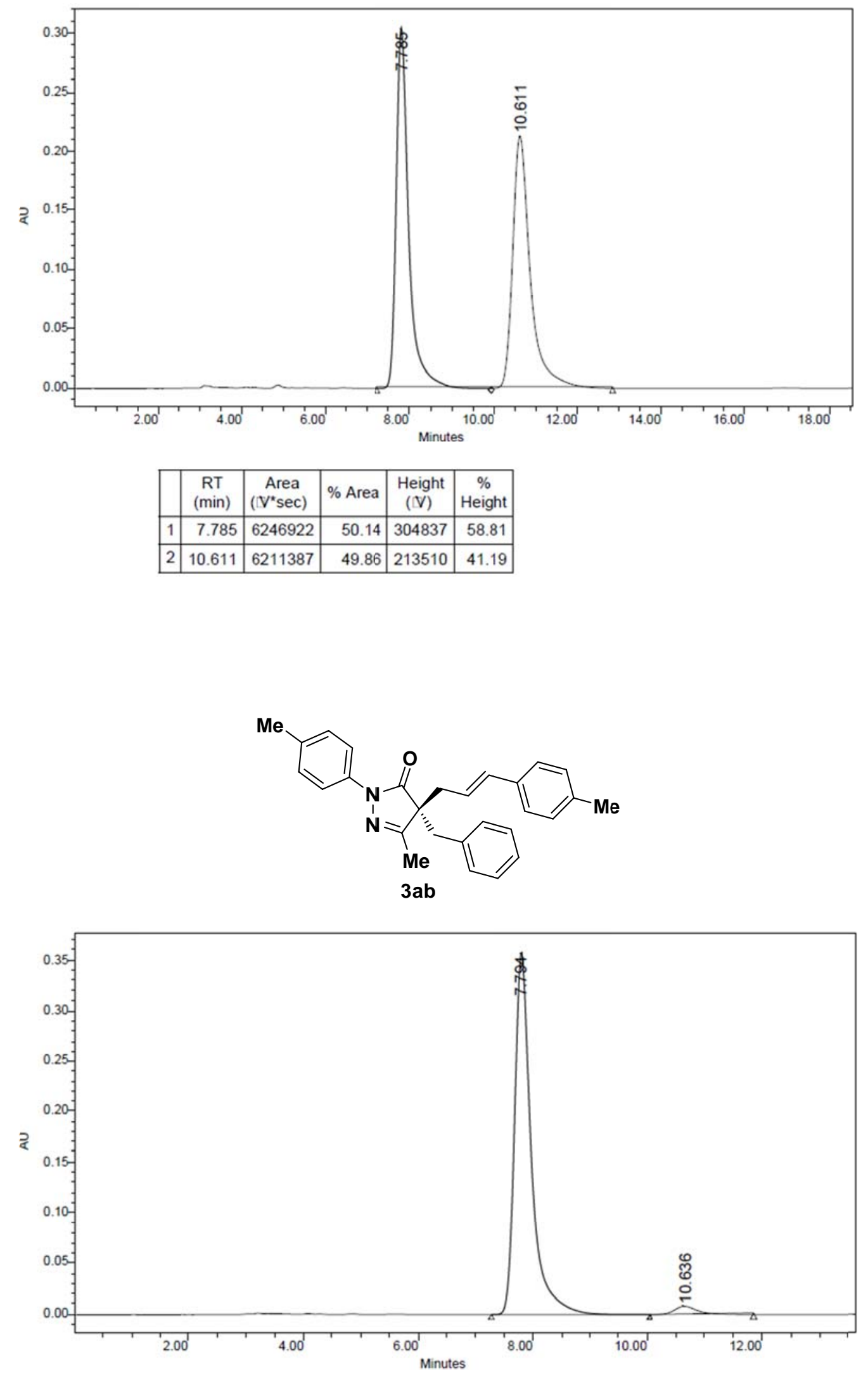

\begin{tabular}{|l|c|c|r|r|r|}
\hline & $\begin{array}{c}\text { RT } \\
(\mathrm{min})\end{array}$ & $\begin{array}{c}\text { Area } \\
\left(\mathrm{V}^{*} \mathrm{sec}\right)\end{array}$ & $\%$ Area & $\begin{array}{c}\text { Height } \\
(\mathrm{V})\end{array}$ & $\begin{array}{c}\% \\
\text { Height }\end{array}$ \\
\hline 1 & 7.794 & 7225680 & 96.90 & 358667 & 97.77 \\
\hline 2 & 10.636 & 230926 & 3.10 & 8177 & 2.23 \\
\hline
\end{tabular}




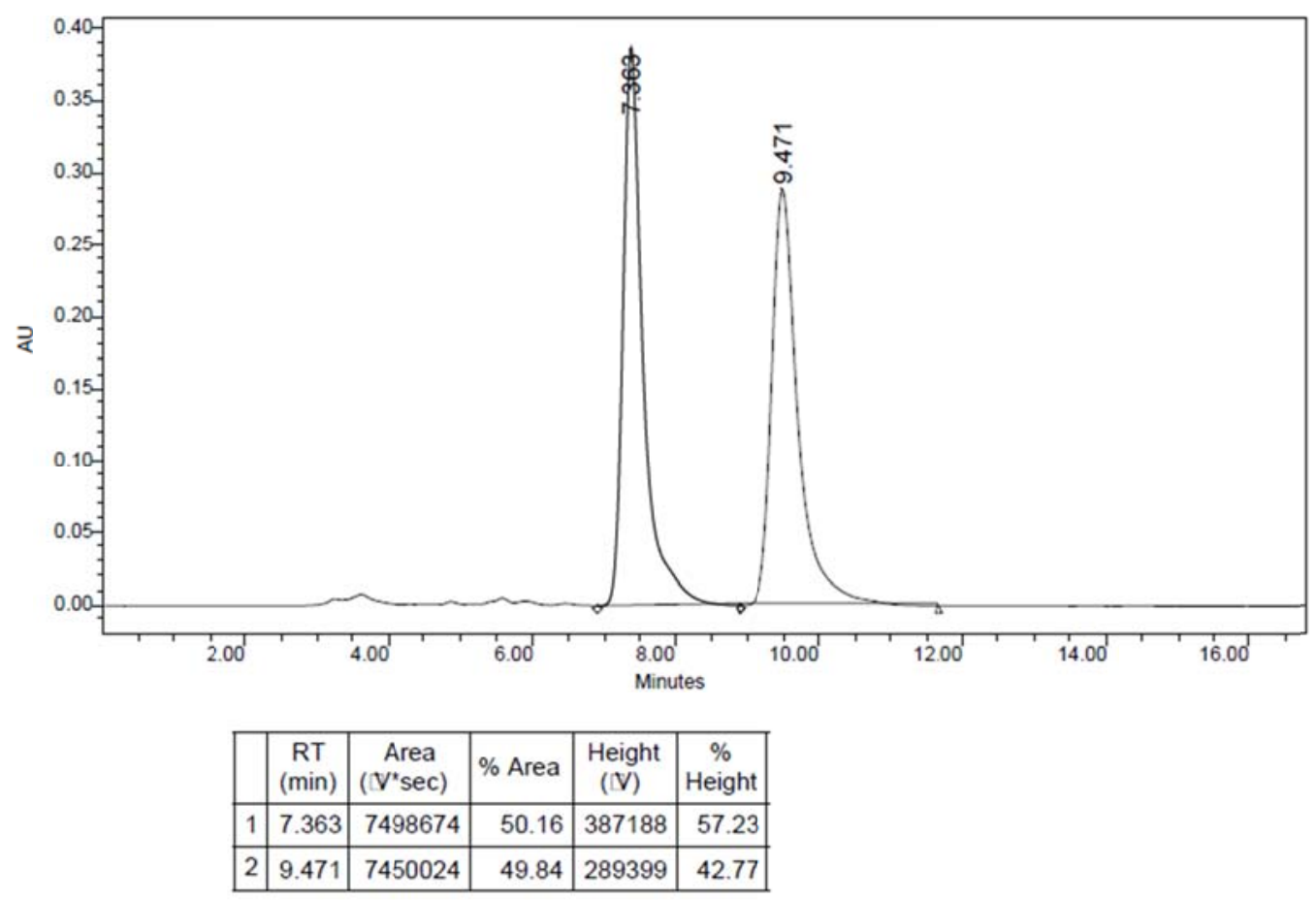

Me<smiles>CC1=NN(c2ccc(F)cc2)C(=O)[C@]1(C/C=C/c1ccc(C(C)(C)C)cc1)Cc1ccccc1</smiles>

3ac

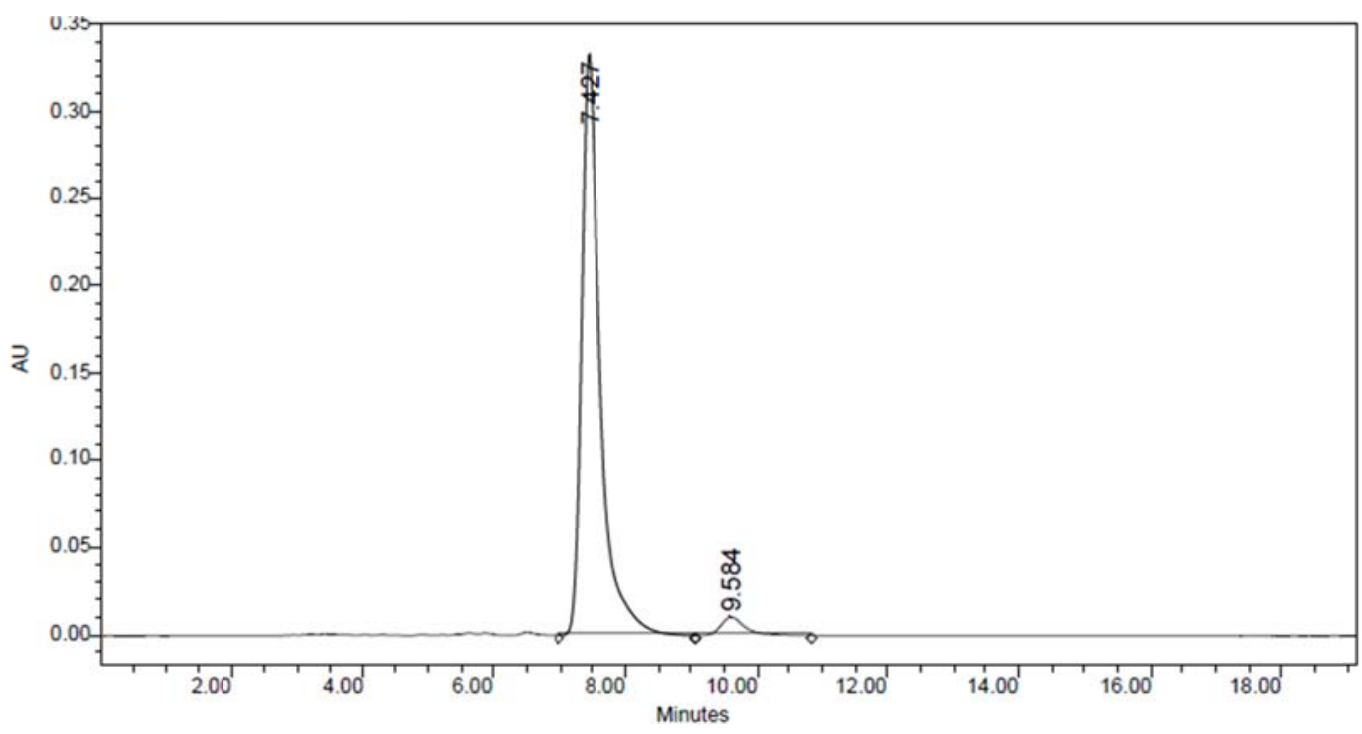

\begin{tabular}{|l|c|r|r|r|r|}
\hline & $\begin{array}{c}\mathrm{RT} \\
(\mathrm{min})\end{array}$ & $\begin{array}{c}\text { Area } \\
\left(\mathrm{V}^{*} \mathrm{sec}\right)\end{array}$ & $\%$ Area & $\begin{array}{l}\text { Height } \\
(\mathrm{V})\end{array}$ & $\begin{array}{c}\% \\
\text { Height }\end{array}$ \\
\hline 1 & 7.427 & 6573251 & 96.03 & 333427 & 96.95 \\
\hline 2 & 9.584 & 271891 & 3.97 & 10484 & 3.05 \\
\hline
\end{tabular}




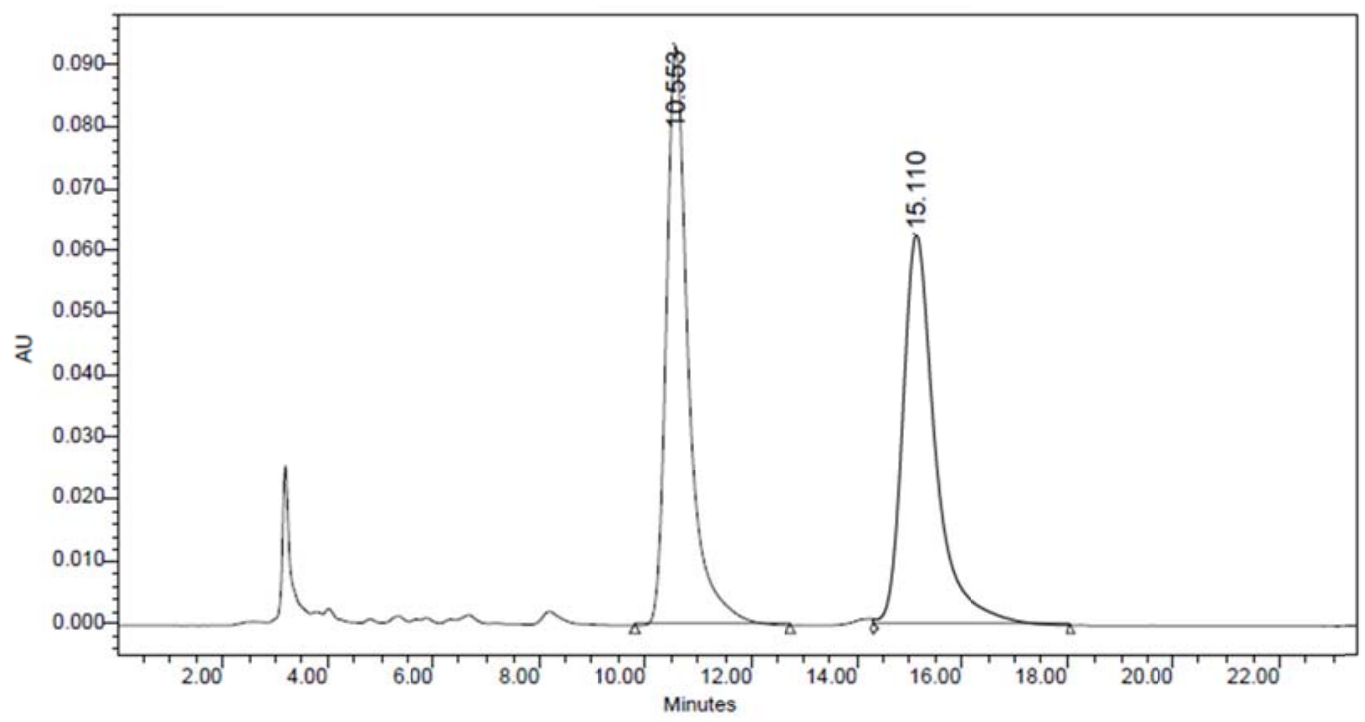

\begin{tabular}{|c|c|c|c|c|c|}
\hline & $\begin{array}{c}\text { RT } \\
(\mathrm{min})\end{array}$ & $\begin{array}{c}\text { Area } \\
(\text { V*sec })\end{array}$ & $\%$ Area & $\begin{array}{c}\text { Height } \\
(\text { V })\end{array}$ & $\begin{array}{c}\% \\
\text { Height }\end{array}$ \\
\hline 1 & 10.553 & 2701417 & 49.95 & 93323 & 59.75 \\
\hline 2 & 15.110 & 2706398 & 50.05 & 62879 & 40.25 \\
\hline
\end{tabular}

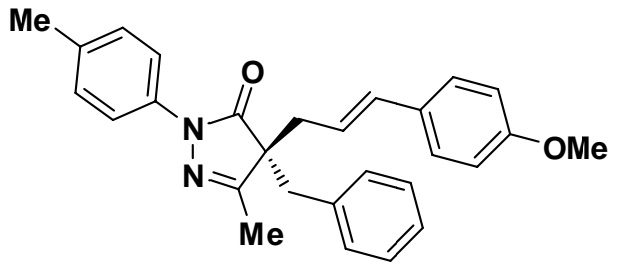

$3 a d$

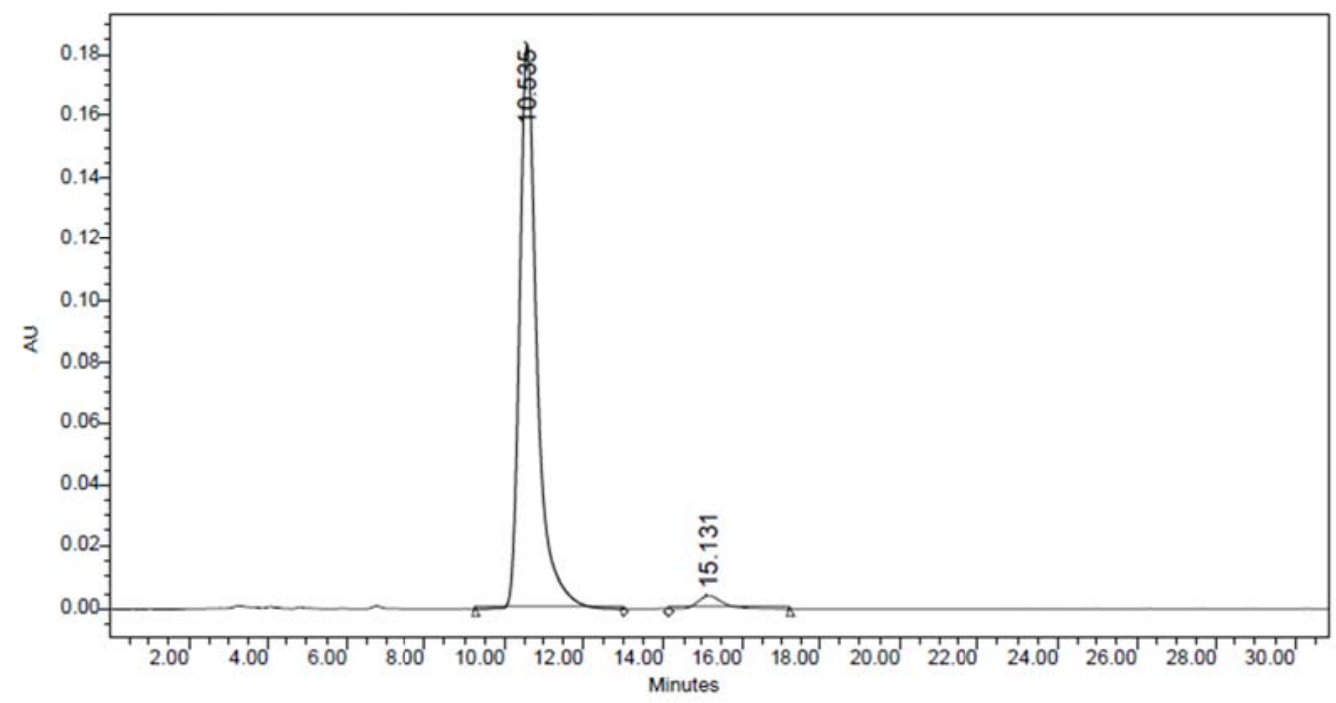

\begin{tabular}{|l|c|c|r|r|r|}
\hline & $\begin{array}{c}\text { RT } \\
(\mathrm{min})\end{array}$ & $\begin{array}{c}\text { Area } \\
\left(\mathrm{V}^{*} \mathrm{sec}\right)\end{array}$ & $\%$ Area & $\begin{array}{c}\text { Height } \\
(\mathrm{V})\end{array}$ & $\begin{array}{c}\% \\
\text { Height }\end{array}$ \\
\hline 1 & 10.535 & 5560553 & 96.67 & 183749 & 97.72 \\
\hline 2 & 15.131 & 191673 & 3.33 & 4294 & 2.28 \\
\hline
\end{tabular}




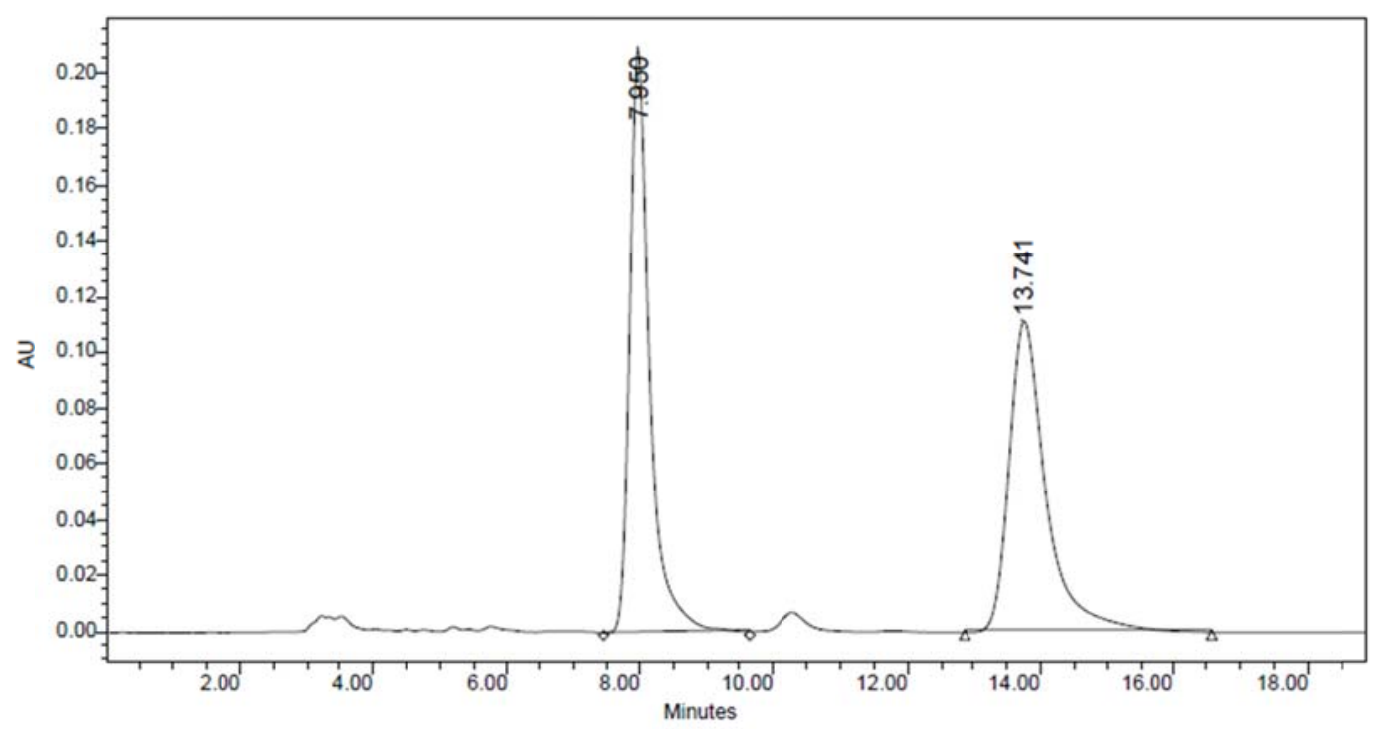

\begin{tabular}{|c|c|c|c|c|c|}
\hline & $\begin{array}{c}\text { RT } \\
(\mathrm{min})\end{array}$ & $\begin{array}{c}\text { Area } \\
\left(\mathrm{V}^{*} \mathrm{sec}\right)\end{array}$ & \% Area & $\begin{array}{c}\text { Height } \\
(\mathrm{V})\end{array}$ & $\begin{array}{c}\% \\
\text { Height }\end{array}$ \\
\hline 1 & 7.950 & 4328404 & 50.11 & 208564 & 65.16 \\
\hline 2 & 13.741 & 4309617 & 49.89 & 111494 & 34.84 \\
\hline
\end{tabular}
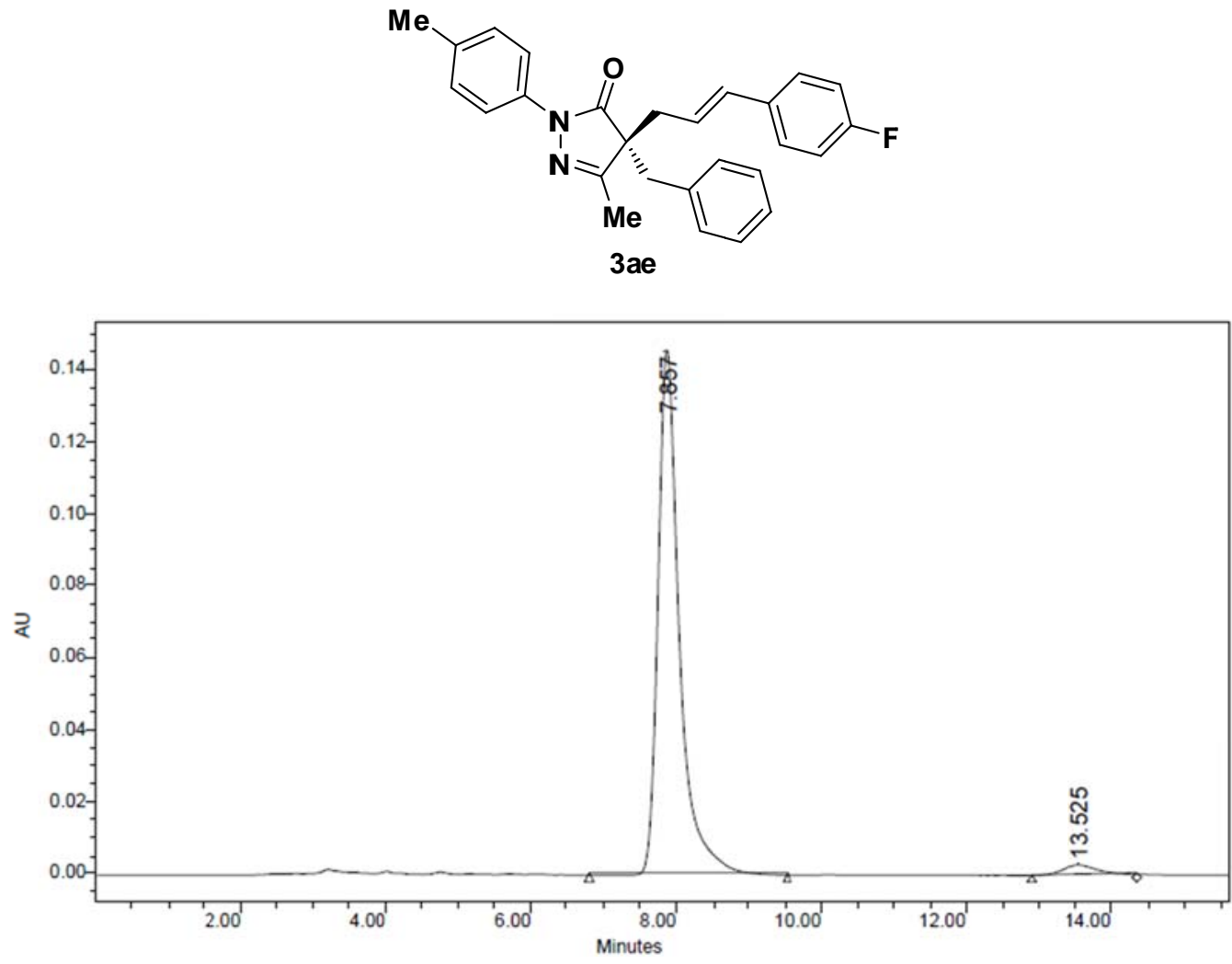

\begin{tabular}{|c|c|r|r|r|r|}
\hline & $\begin{array}{c}\text { RT } \\
(\mathrm{min})\end{array}$ & $\begin{array}{c}\text { Area } \\
\left(\mathbb{V}^{*} \mathrm{sec}\right)\end{array}$ & \% Area & $\begin{array}{c}\text { Height } \\
(\mathrm{V})\end{array}$ & $\begin{array}{c}\% \\
\text { Height }\end{array}$ \\
\hline 1 & 7.857 & 2970192 & 96.82 & 145887 & 98.12 \\
\hline 2 & 13.525 & 97611 & 3.18 & 2793 & 1.88 \\
\hline
\end{tabular}




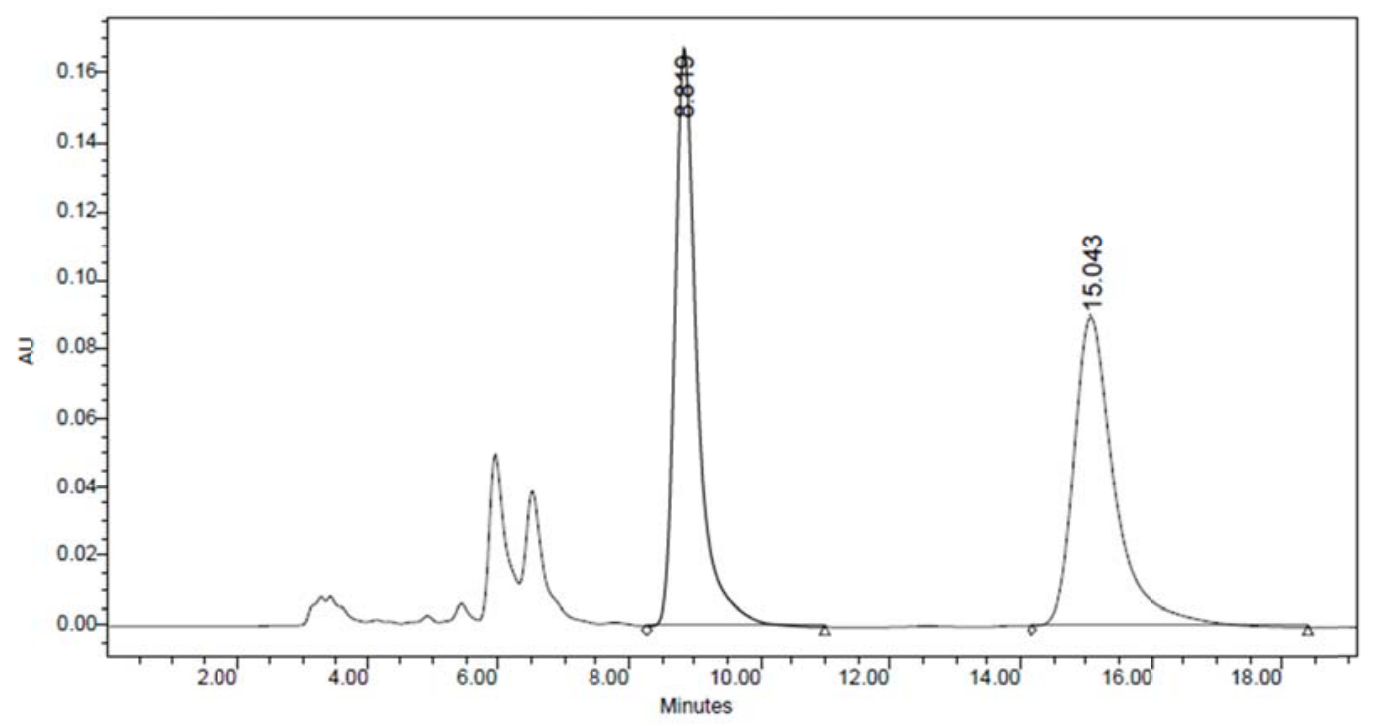

\begin{tabular}{|c|c|c|c|c|c|}
\hline & $\begin{array}{c}\mathrm{RT} \\
(\mathrm{min})\end{array}$ & $\begin{array}{c}\text { Area } \\
\left(\mathrm{V}^{*} \mathrm{sec}\right)\end{array}$ & \% Area & $\begin{array}{c}\text { Height } \\
(\mathrm{V})\end{array}$ & $\begin{array}{c}\% \\
\text { Height }\end{array}$ \\
\hline 1 & 8.819 & 3971187 & 50.19 & 167640 & 65.11 \\
\hline 2 & 15.043 & 3940651 & 49.81 & 89826 & 34.89 \\
\hline
\end{tabular}
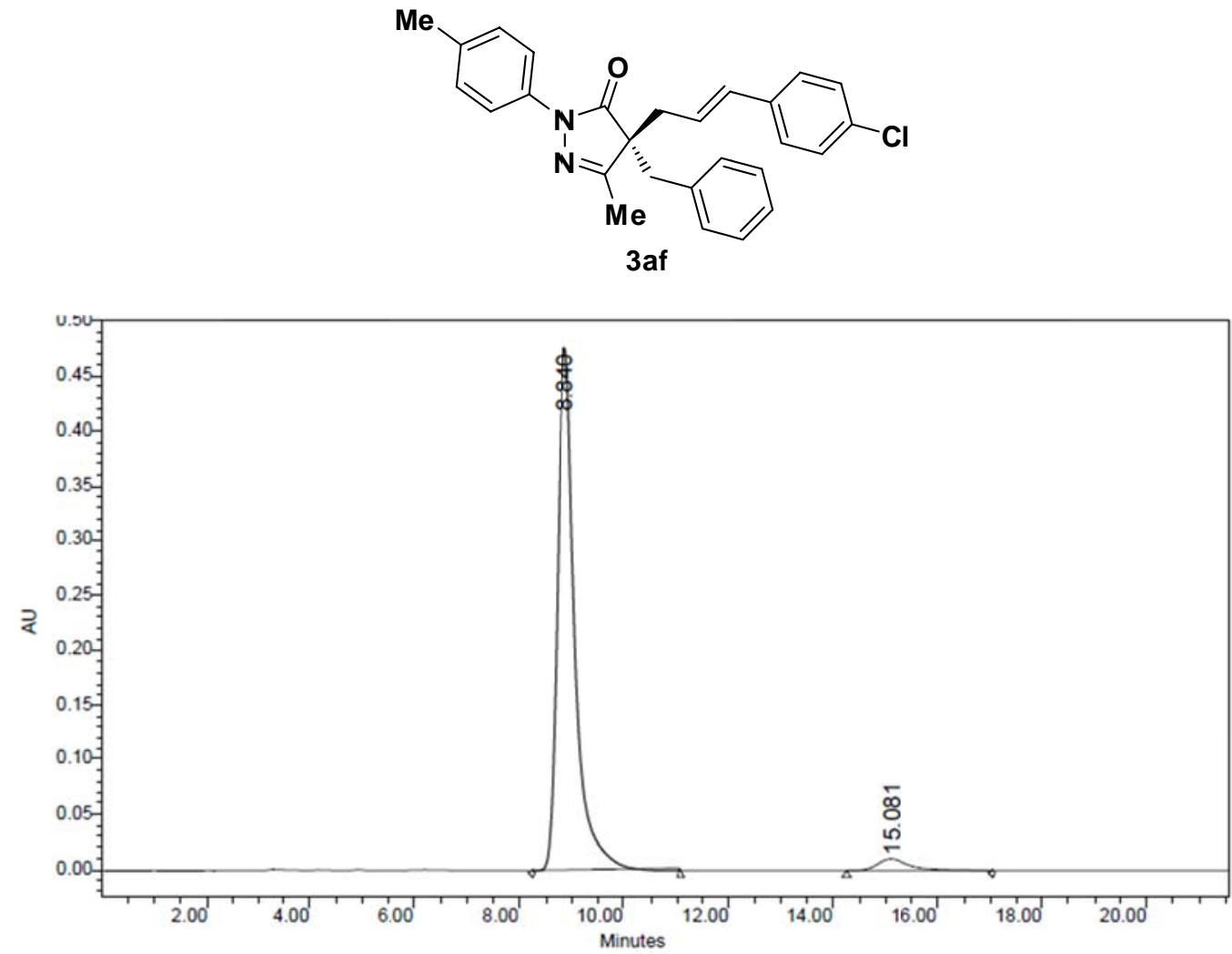

\begin{tabular}{|c|c|c|r|r|r|}
\hline & $\begin{array}{c}\mathrm{RT} \\
(\mathrm{min})\end{array}$ & $\begin{array}{c}\text { Area } \\
\left(\mathrm{V}^{*} \mathrm{sec}\right)\end{array}$ & $\%$ Area & $\begin{array}{c}\text { Height } \\
(\mathrm{V})\end{array}$ & $\begin{array}{c}\% \\
\text { Height }\end{array}$ \\
\hline 1 & 8.840 & 11133821 & 96.00 & 476386 & 97.80 \\
\hline 2 & 15.081 & 464469 & 4.00 & 10699 & 2.20 \\
\hline
\end{tabular}



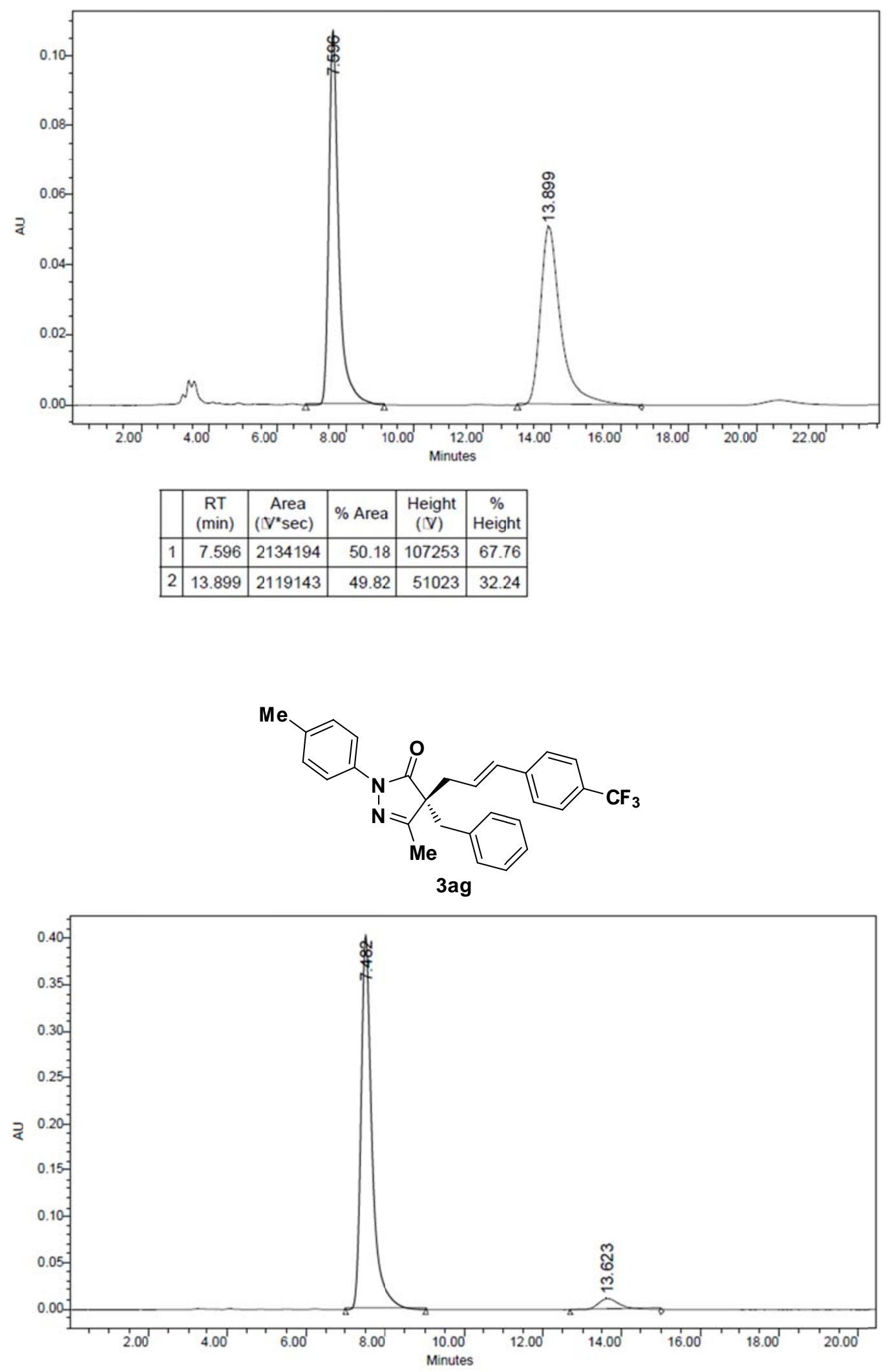

\begin{tabular}{|c|c|c|r|r|r|}
\hline & $\begin{array}{c}\text { RT } \\
(\mathrm{min})\end{array}$ & $\begin{array}{c}\text { Area } \\
\left(\mathrm{V}^{*} \mathrm{sec}\right)\end{array}$ & \% Area & $\begin{array}{c}\text { Height } \\
(\mathrm{V})\end{array}$ & $\begin{array}{c}\% \\
\text { Height }\end{array}$ \\
\hline 1 & 7.482 & 7796493 & 94.42 & 403758 & 97.16 \\
\hline 2 & 13.623 & 460741 & 5.58 & 11811 & 2.84 \\
\hline
\end{tabular}



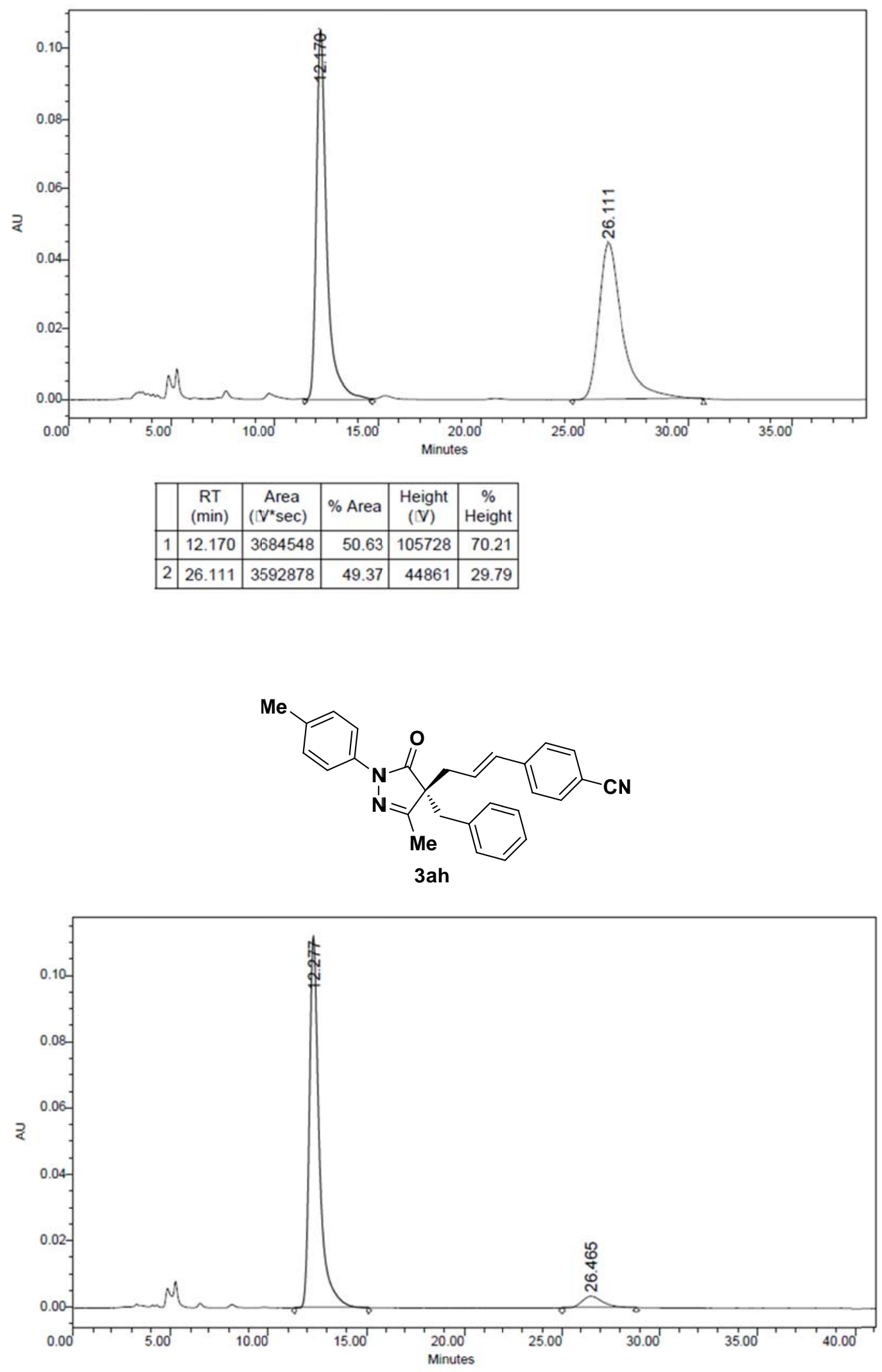

\begin{tabular}{|c|c|c|r|r|r|}
\hline & $\begin{array}{c}\text { RT } \\
(\mathrm{min})\end{array}$ & $\begin{array}{c}\text { Area } \\
\left(\mathrm{V}^{*} \mathrm{sec}\right)\end{array}$ & \% Area & $\begin{array}{c}\text { Height } \\
(\mathrm{V})\end{array}$ & $\begin{array}{c}\% \\
\text { Height }\end{array}$ \\
\hline 1 & 12.277 & 3917805 & 93.61 & 111831 & 96.96 \\
\hline 2 & 26.465 & 267507 & 6.39 & 3501 & 3.04 \\
\hline
\end{tabular}




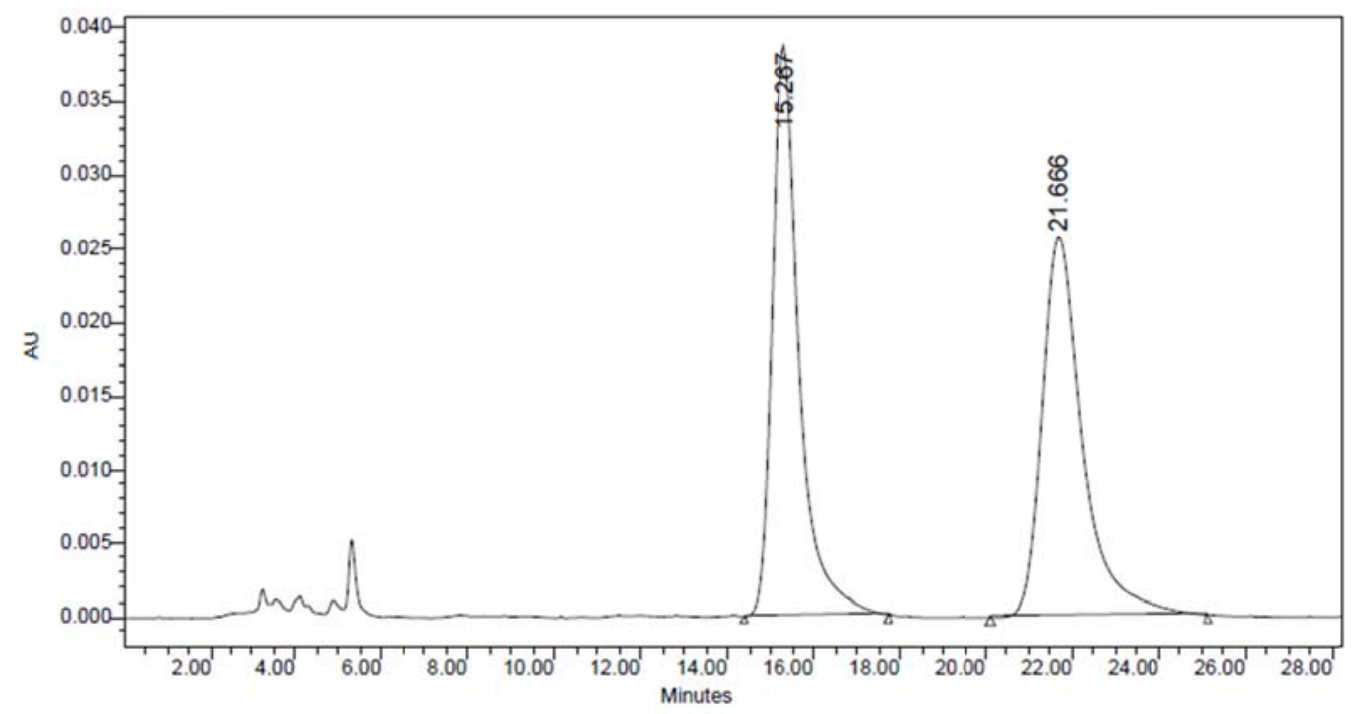

\begin{tabular}{|c|c|c|c|c|c|}
\hline & $\begin{array}{c}\text { RT } \\
(\mathrm{min})\end{array}$ & $\begin{array}{c}\text { Area } \\
\left(\text { V*sec }^{*}\right.\end{array}$ & $\%$ Area & $\begin{array}{c}\text { Height } \\
(\text { V })\end{array}$ & $\begin{array}{c}\% \\
\text { Height }\end{array}$ \\
\hline 1 & 15.267 & 1687884 & 50.23 & 38568 & 59.91 \\
\hline 2 & 21.666 & 1672338 & 49.77 & 25804 & 40.09 \\
\hline
\end{tabular}

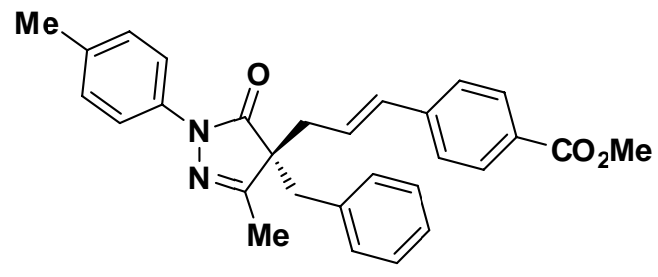

3ai

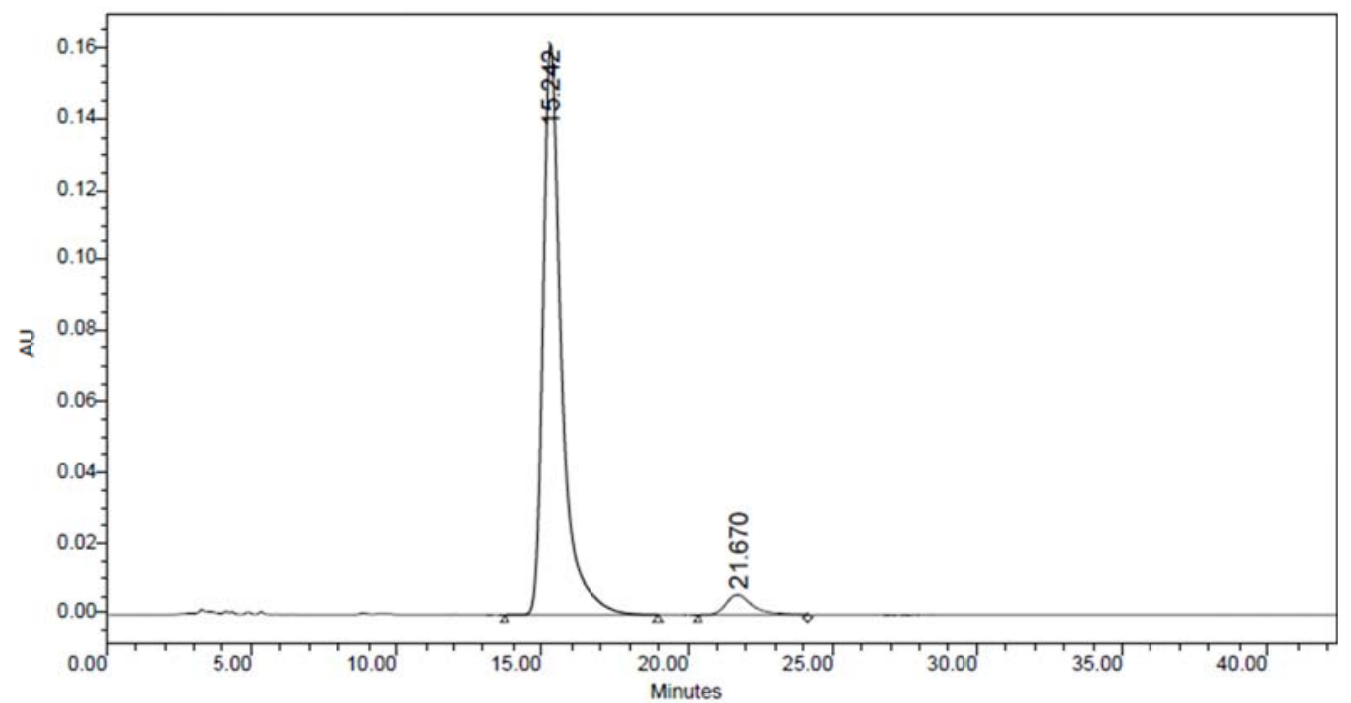

\begin{tabular}{|l|c|c|r|r|r|}
\hline & $\begin{array}{c}\text { RT } \\
(\mathrm{min})\end{array}$ & $\begin{array}{c}\text { Area } \\
\left(\mathrm{V}^{*} \mathrm{sec}\right)\end{array}$ & $\%$ Area & $\begin{array}{c}\text { Height } \\
(\mathrm{V})\end{array}$ & $\begin{array}{c}\% \\
\text { Height }\end{array}$ \\
\hline 1 & 15.242 & 7137297 & 94.97 & 161404 & 96.52 \\
\hline 2 & 21.670 & 377715 & 5.03 & 5819 & 3.48 \\
\hline
\end{tabular}




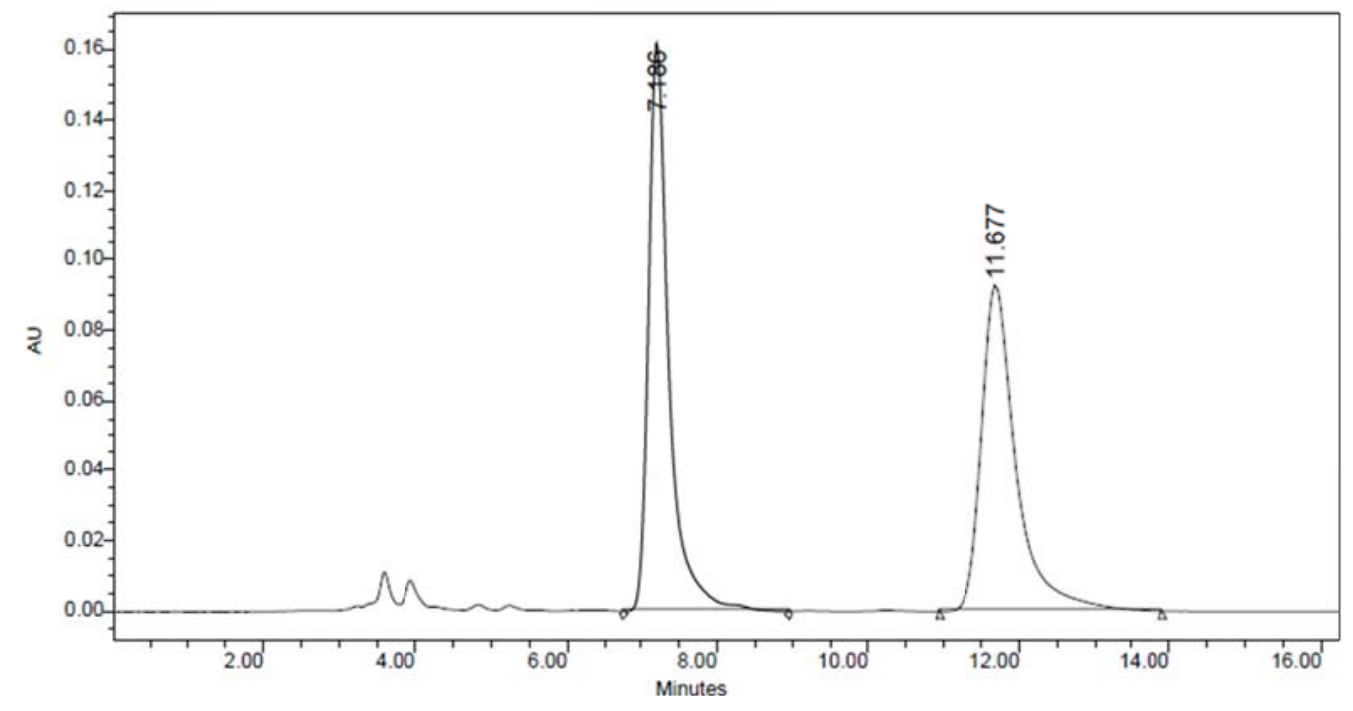

\begin{tabular}{|c|c|c|c|c|c|}
\hline & $\begin{array}{c}\text { RT } \\
(\mathrm{min})\end{array}$ & $\begin{array}{c}\text { Area } \\
\left(\mathrm{V}^{*} \mathrm{sec}\right)\end{array}$ & $\%$ Area & $\begin{array}{c}\text { Height } \\
(\mathrm{V})\end{array}$ & $\begin{array}{c}\% \\
\text { Height }\end{array}$ \\
\hline 1 & 7.186 & 3039980 & 50.50 & 162145 & 63.57 \\
\hline 2 & 11.677 & 2979650 & 49.50 & 92903 & 36.43 \\
\hline
\end{tabular}
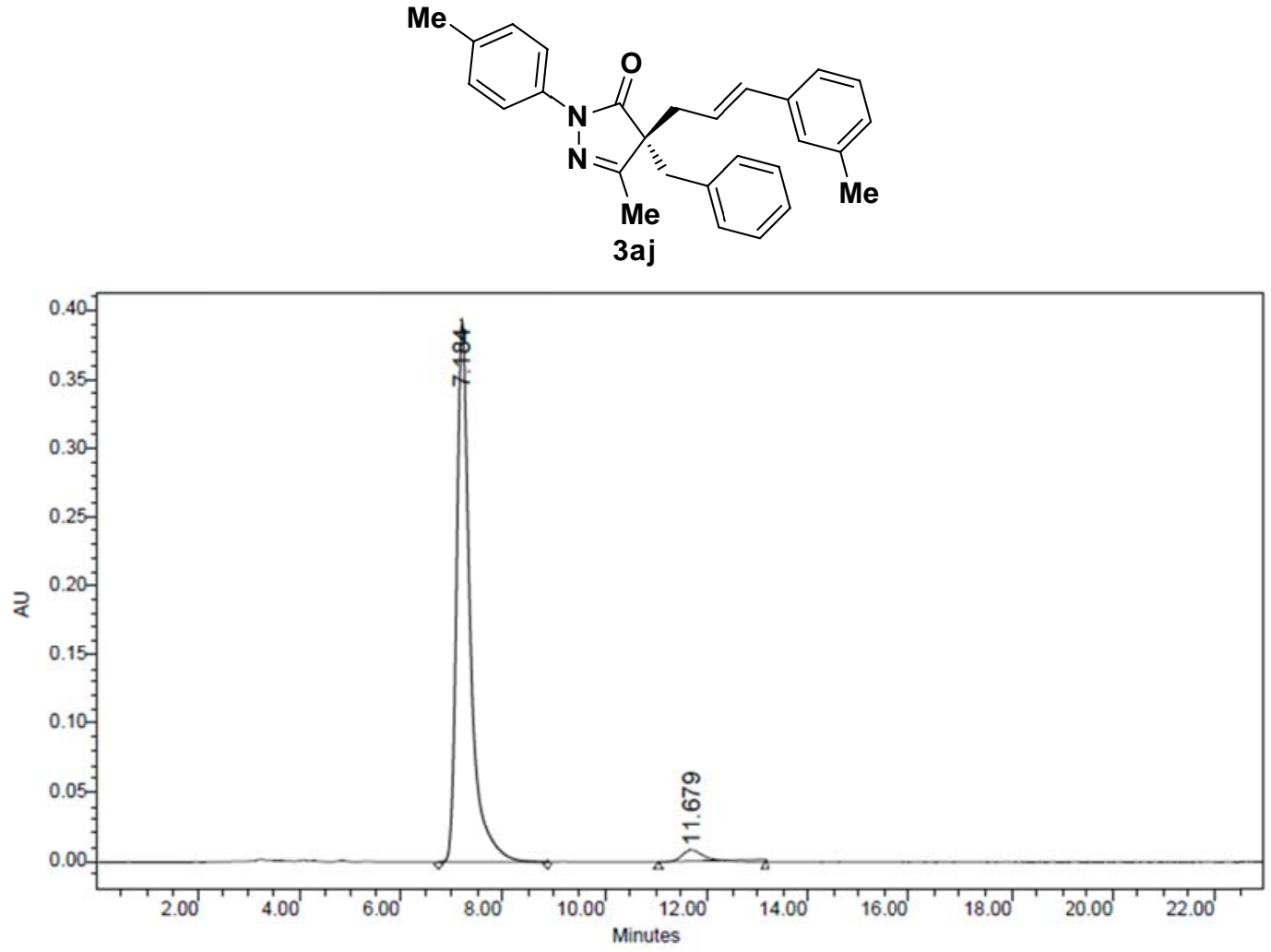

\begin{tabular}{|c|c|c|r|r|r|}
\hline & $\begin{array}{c}\text { RT } \\
(\mathrm{min})\end{array}$ & $\begin{array}{c}\text { Area } \\
\left(\mathrm{V}^{*} \mathrm{sec}\right)\end{array}$ & \% Area & $\begin{array}{c}\text { Height } \\
(\mathrm{V})\end{array}$ & $\begin{array}{c}\% \\
\text { Height }\end{array}$ \\
\hline 1 & 7.184 & 7219080 & 96.25 & 392306 & 97.84 \\
\hline 2 & 11.679 & 280884 & 3.75 & 8667 & 2.16 \\
\hline
\end{tabular}




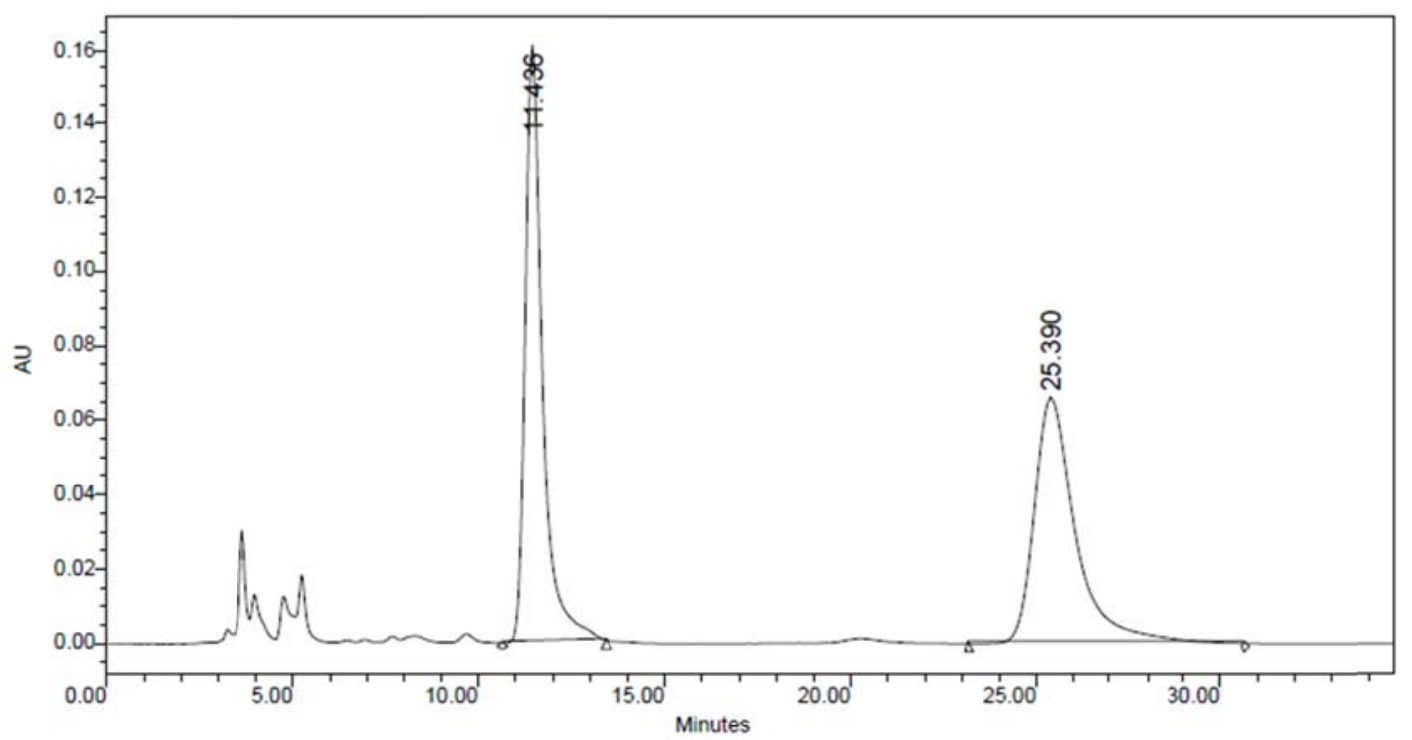

\begin{tabular}{|c|c|c|c|c|c|}
\hline & $\begin{array}{c}\text { RT } \\
(\mathrm{min})\end{array}$ & $\begin{array}{c}\text { Area } \\
\left(\mathrm{V}^{*} \mathrm{sec}\right)\end{array}$ & $\%$ Area & $\begin{array}{c}\text { Height } \\
(\mathrm{V})\end{array}$ & $\begin{array}{c}\% \\
\text { Height }\end{array}$ \\
\hline 1 & 11.436 & 5165836 & 50.26 & 160230 & 70.79 \\
\hline 2 & 25.390 & 5112352 & 49.74 & 66113 & 29.21 \\
\hline
\end{tabular}
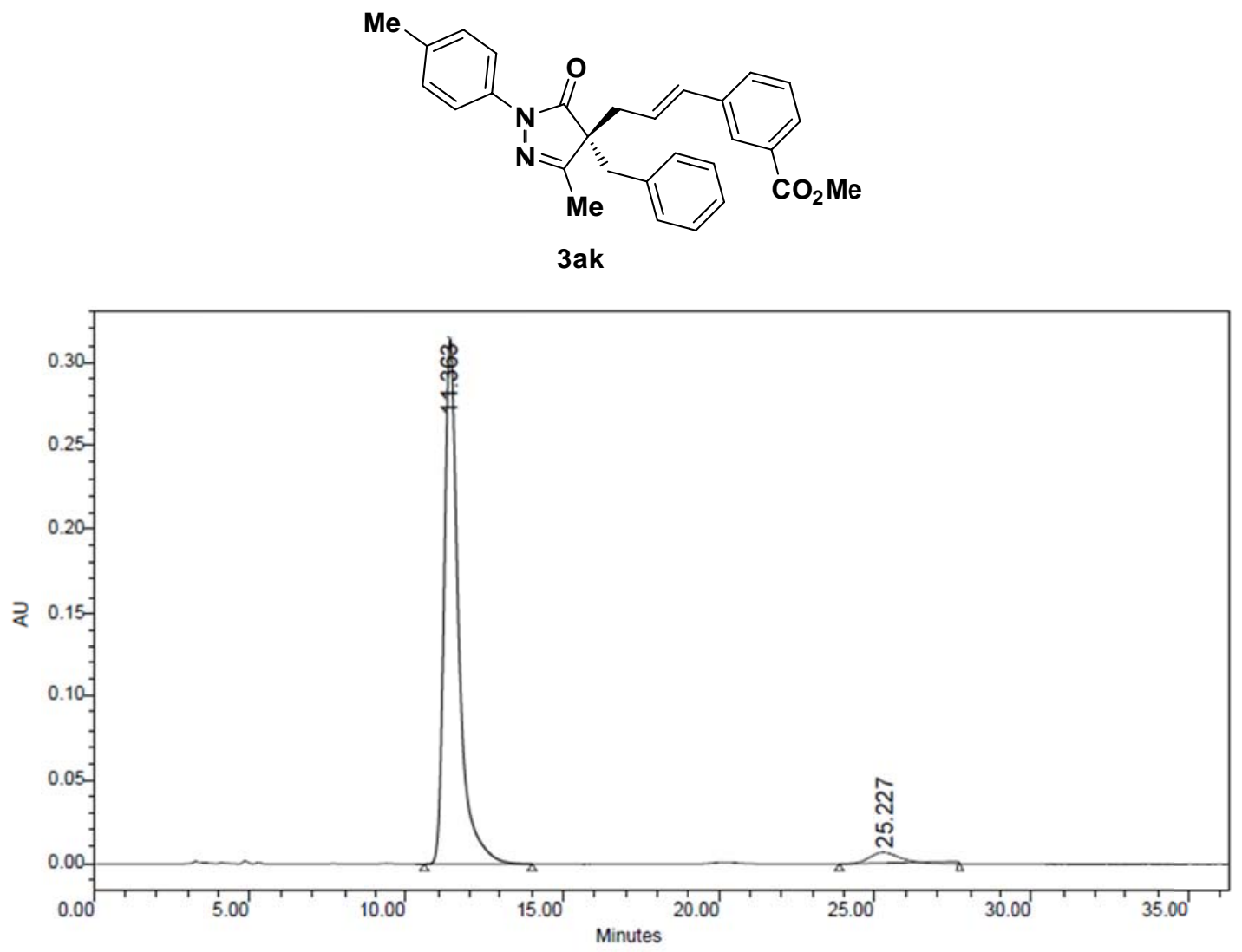

\begin{tabular}{|l|c|r|r|r|r|}
\hline & $\begin{array}{c}\text { RT } \\
(\mathrm{min})\end{array}$ & $\begin{array}{c}\text { Area } \\
\left(\mathrm{V}^{*} \mathrm{sec}\right)\end{array}$ & \% Area & $\begin{array}{c}\text { Height } \\
(\mathrm{V})\end{array}$ & $\begin{array}{c}\% \\
\text { Height }\end{array}$ \\
\hline 1 & 11.363 & 10045454 & 95.36 & 314677 & 97.85 \\
\hline 2 & 25.227 & 488557 & 4.64 & 6922 & 2.15 \\
\hline
\end{tabular}




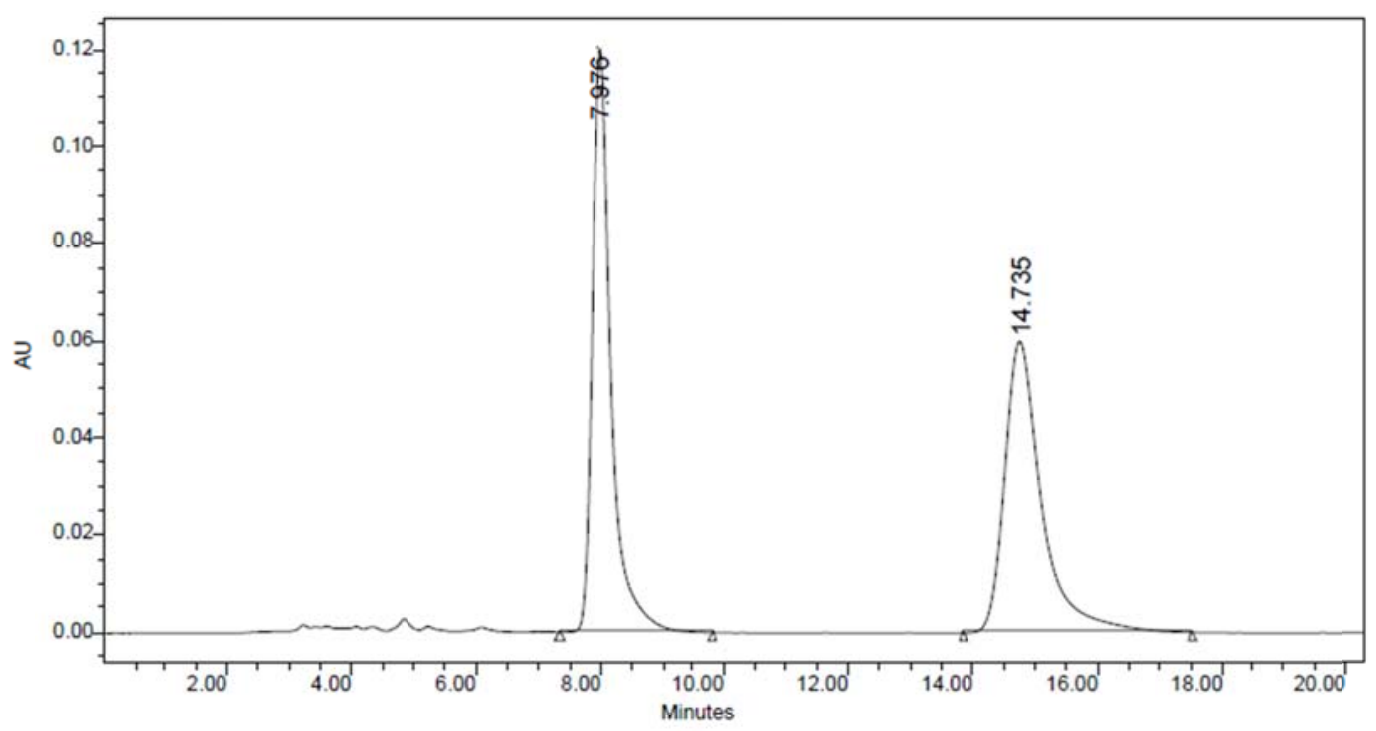

\begin{tabular}{|c|c|c|c|c|c|}
\hline & $\begin{array}{c}\text { RT } \\
(\mathrm{min})\end{array}$ & $\begin{array}{c}\text { Area } \\
\left(\mathrm{V}^{*} \mathrm{sec}\right)\end{array}$ & $\%$ Area & $\begin{array}{c}\text { Height } \\
(\mathrm{V})\end{array}$ & $\begin{array}{c}\% \\
\text { Height }\end{array}$ \\
\hline 1 & 7.976 & 2510048 & 50.32 & 120211 & 66.74 \\
\hline 2 & 14.735 & 2477961 & 49.68 & 59900 & 33.26 \\
\hline
\end{tabular}
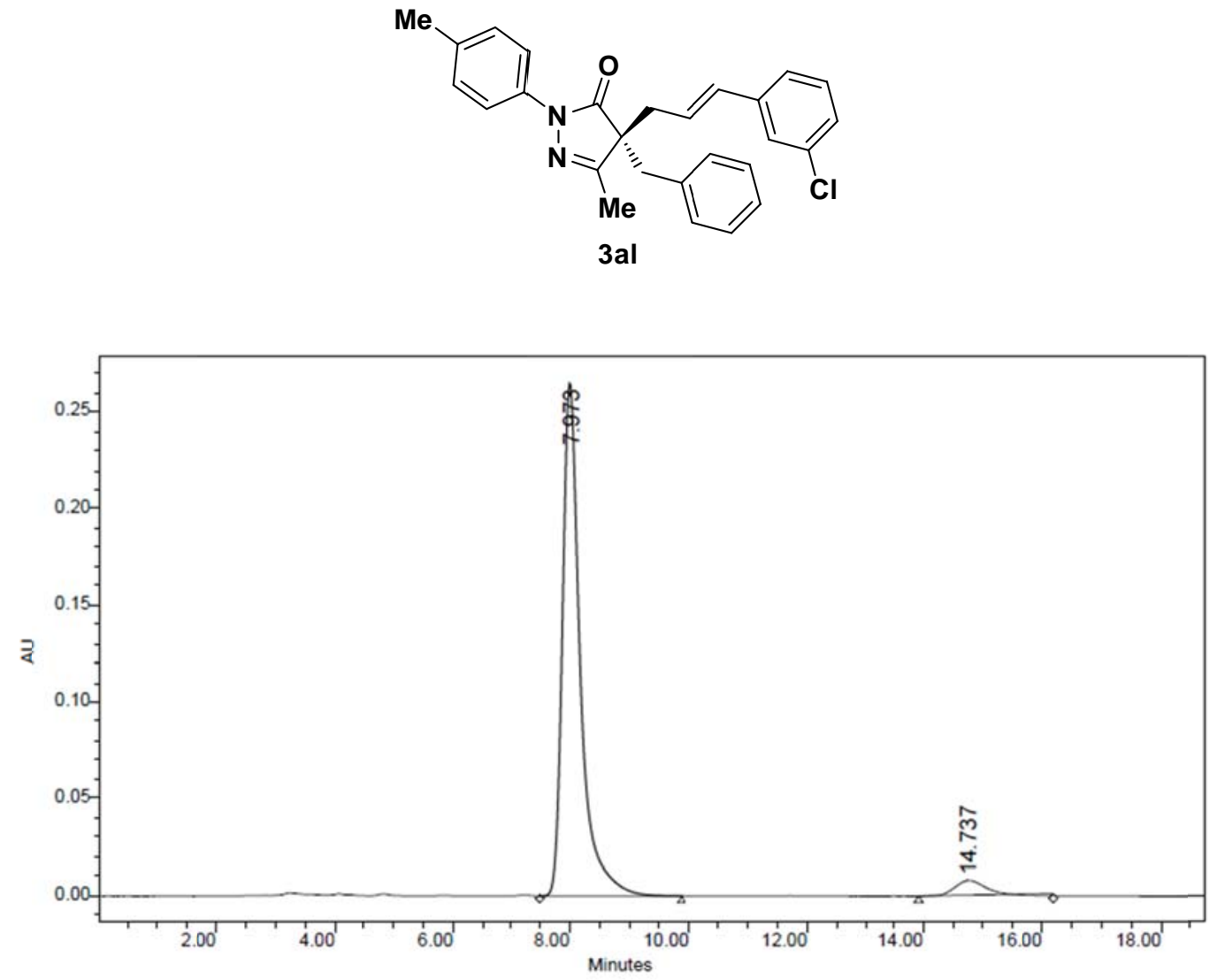

\begin{tabular}{|c|c|c|r|r|r|}
\hline & $\begin{array}{c}\text { RT } \\
(\mathrm{min})\end{array}$ & $\begin{array}{c}\text { Area } \\
\left(\mathrm{V}^{*} \mathrm{sec}\right)\end{array}$ & \% Area & $\begin{array}{c}\text { Height } \\
(\mathrm{V})\end{array}$ & $\begin{array}{c}\% \\
\text { Height }\end{array}$ \\
\hline 1 & 7.973 & 5528617 & 94.51 & 265507 & 97.05 \\
\hline 2 & 14.737 & 321190 & 5.49 & 8061 & 2.95 \\
\hline
\end{tabular}




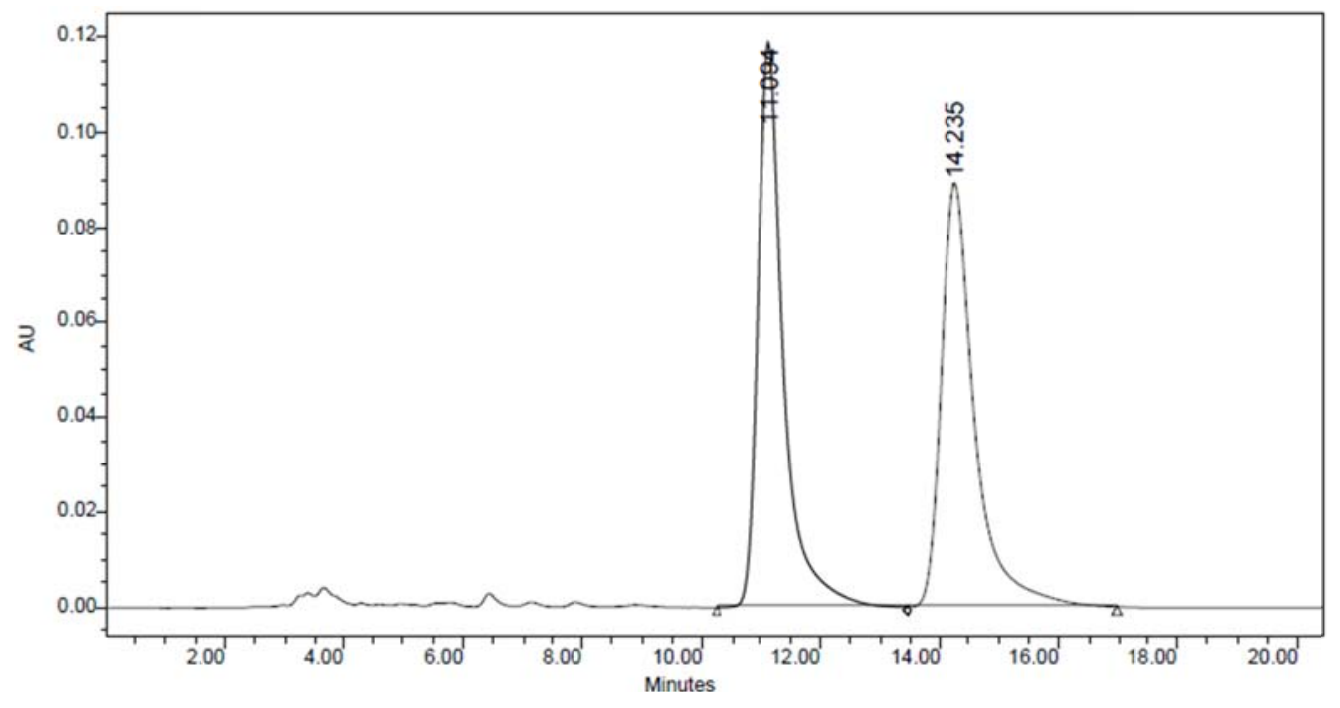

\begin{tabular}{|c|c|c|c|c|c|}
\hline & $\begin{array}{c}\text { RT } \\
(\mathrm{min})\end{array}$ & $\begin{array}{c}\text { Area } \\
\left(\mathrm{V}^{*} \mathrm{sec}\right)\end{array}$ & $\%$ Area & $\begin{array}{c}\text { Height } \\
(\mathrm{V})\end{array}$ & $\begin{array}{c}\% \\
\text { Height }\end{array}$ \\
\hline 1 & 11.094 & 3552674 & 50.49 & 118827 & 57.06 \\
\hline 2 & 14.235 & 3483936 & 49.51 & 89431 & 42.94 \\
\hline
\end{tabular}
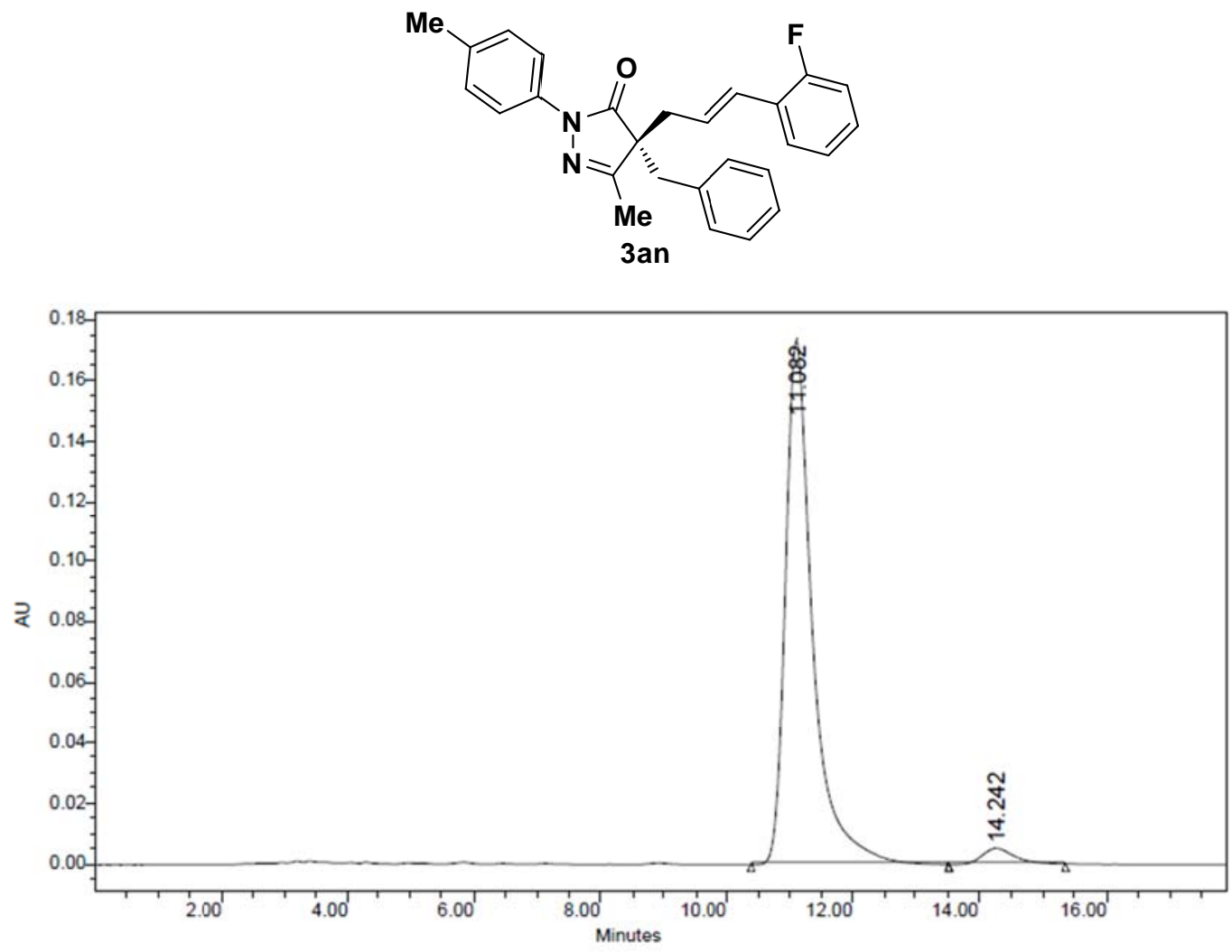

\begin{tabular}{|c|c|c|r|r|r|}
\hline & $\begin{array}{c}\text { RT } \\
(\mathrm{min})\end{array}$ & $\begin{array}{c}\text { Area } \\
\left(\mathrm{V}^{*} \mathrm{sec}\right)\end{array}$ & \% Area & $\begin{array}{c}\text { Height } \\
(\mathrm{V})\end{array}$ & $\begin{array}{c}\% \\
\text { Height }\end{array}$ \\
\hline 1 & 11.082 & 5184519 & 96.57 & 173538 & 97.07 \\
\hline 2 & 14.242 & 184167 & 3.43 & 5240 & 2.93 \\
\hline
\end{tabular}




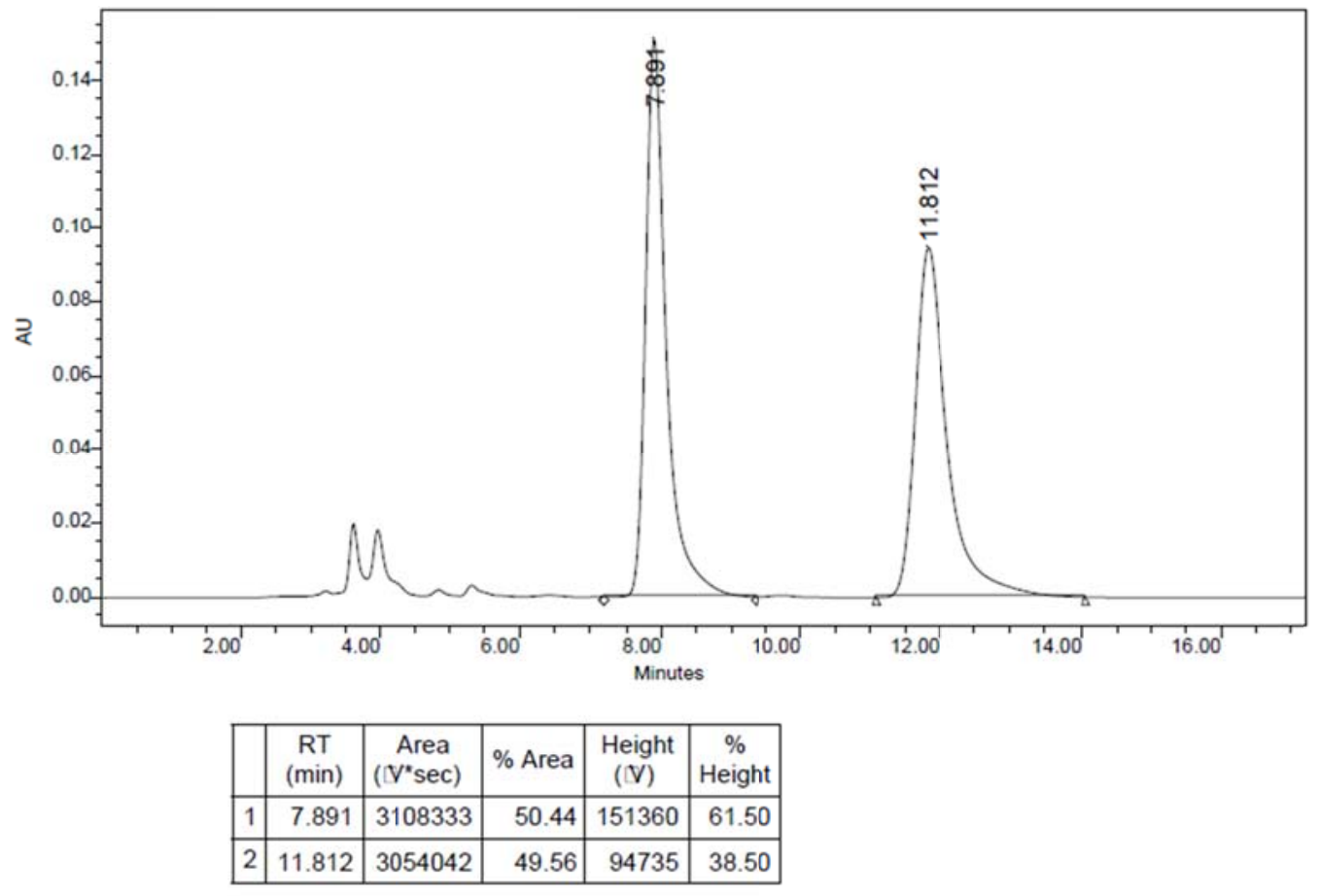

(n)

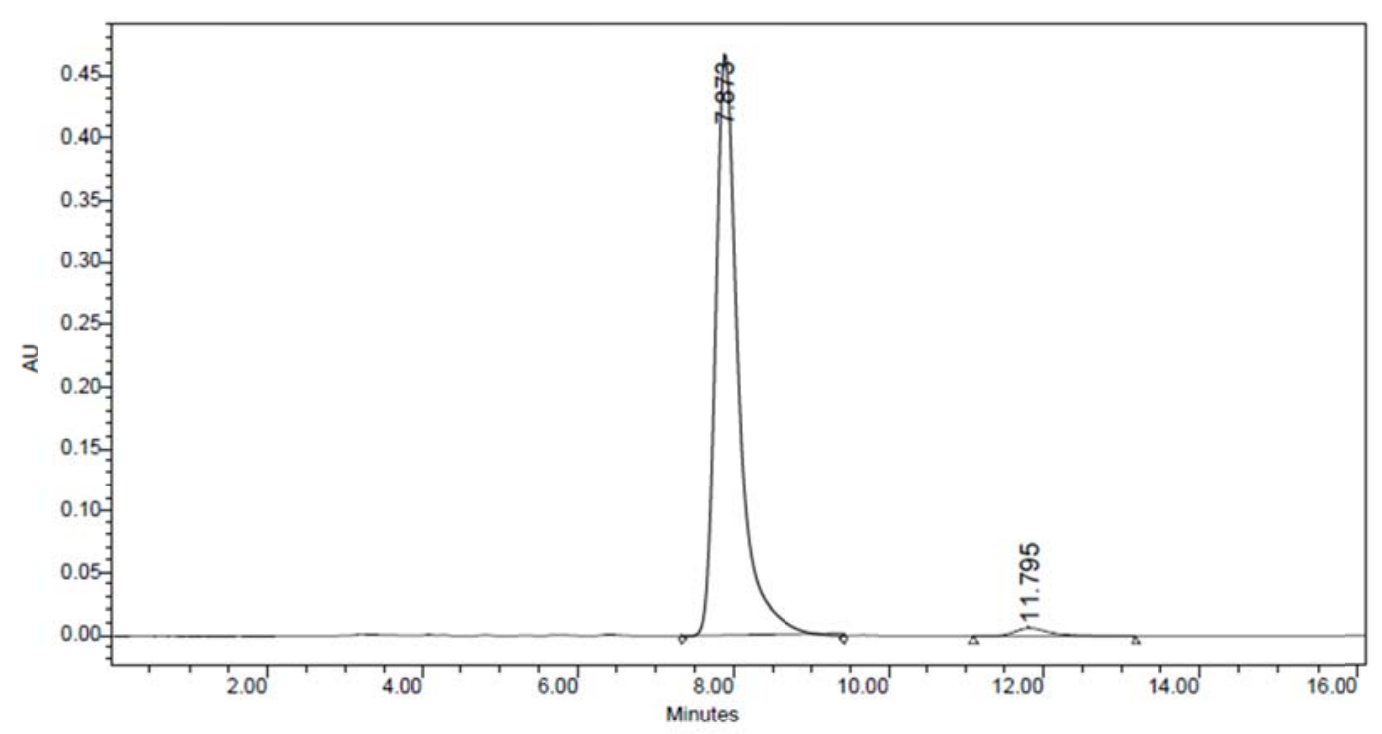

\begin{tabular}{|c|c|c|r|r|r|}
\hline & $\begin{array}{c}\text { RT } \\
(\mathrm{min})\end{array}$ & $\begin{array}{c}\text { Area } \\
\left(\mathrm{V}^{*} \mathrm{sec}\right)\end{array}$ & $\%$ Area & $\begin{array}{c}\text { Height } \\
(\mathrm{V})\end{array}$ & $\begin{array}{c}\% \\
\text { Height }\end{array}$ \\
\hline 1 & 7.873 & 9531484 & 97.95 & 467946 & 98.65 \\
\hline 2 & 11.795 & 199010 & 2.05 & 6402 & 1.35 \\
\hline
\end{tabular}



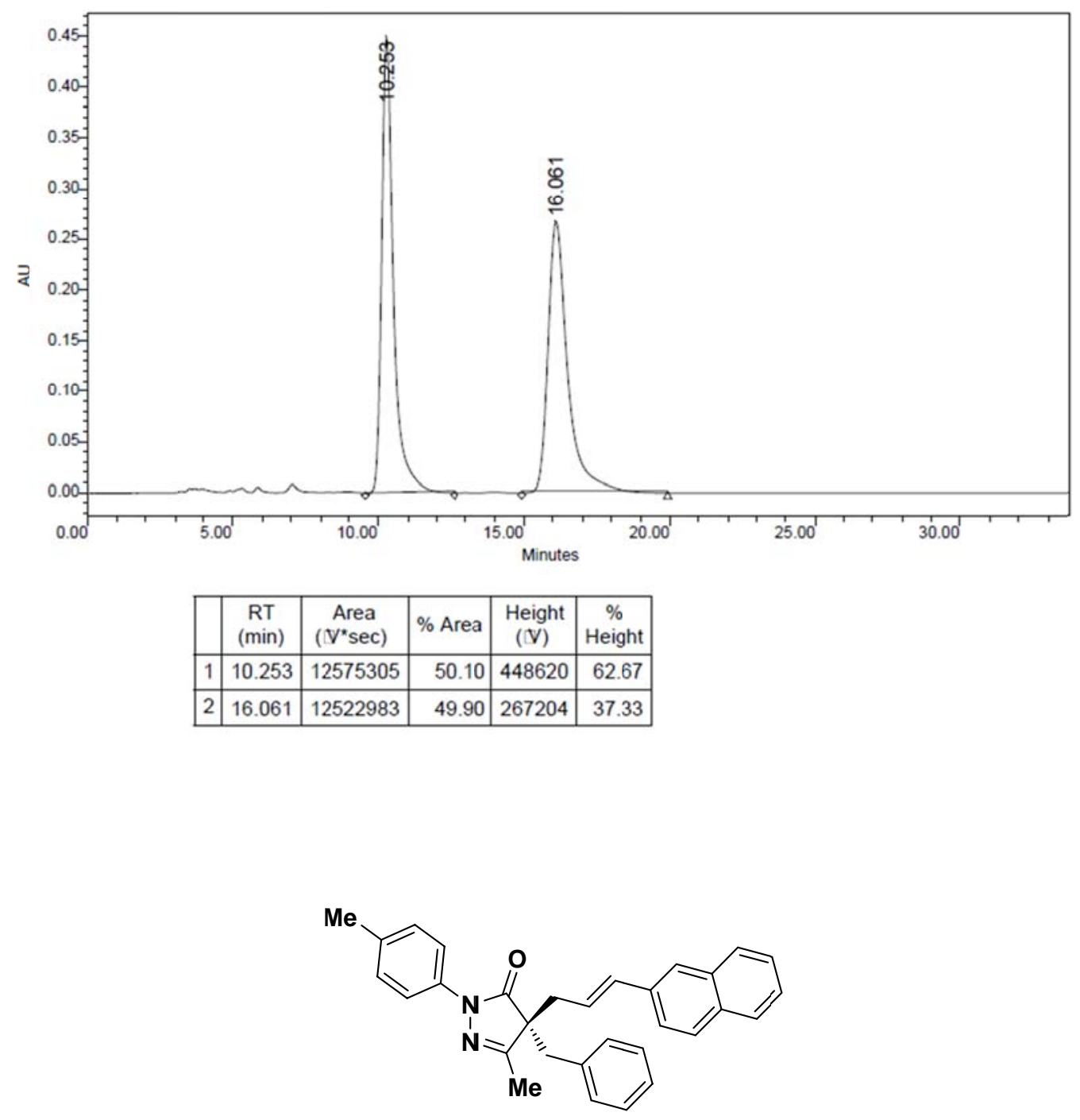

$3 a o$

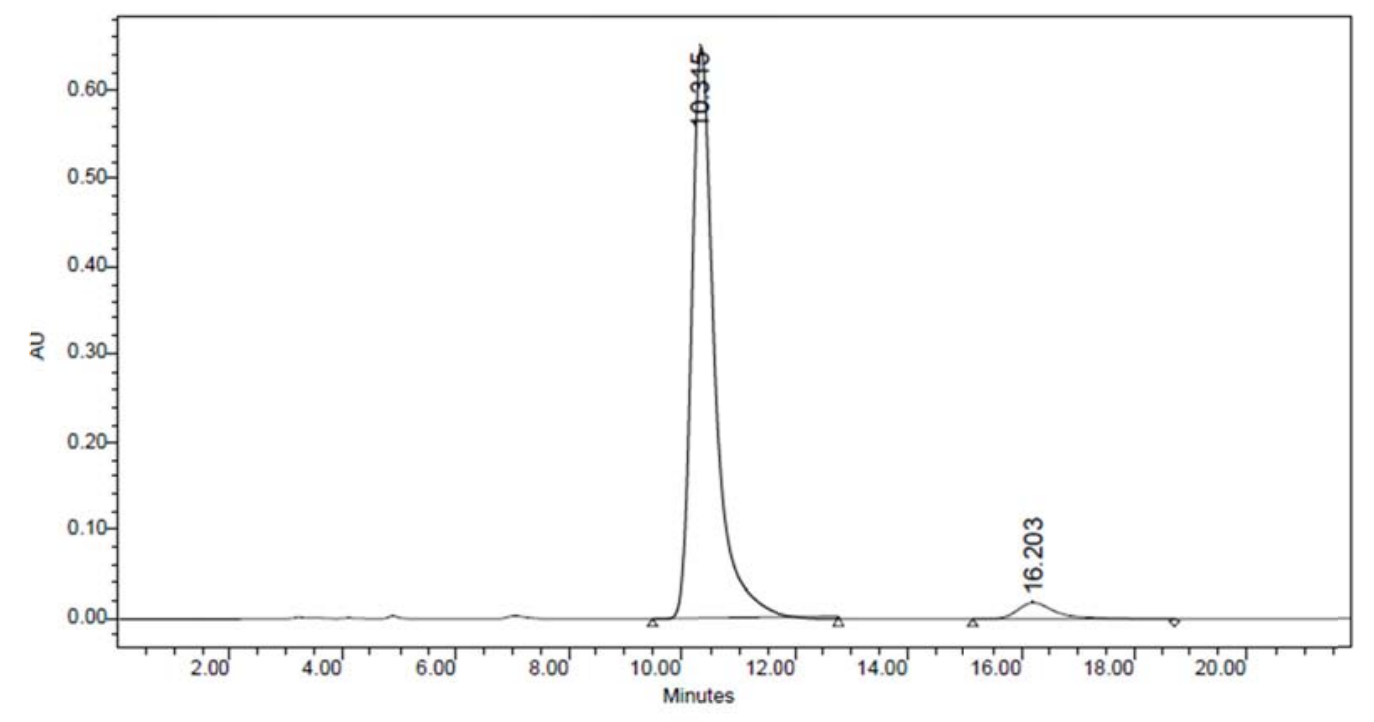

\begin{tabular}{|c|c|c|r|r|r|}
\hline & $\begin{array}{c}\mathrm{RT} \\
(\mathrm{min})\end{array}$ & $\begin{array}{c}\text { Area } \\
\left(\mathrm{V}^{*} \mathrm{sec}\right)\end{array}$ & $\%$ Area & $\begin{array}{c}\text { Height } \\
(\mathrm{V})\end{array}$ & $\begin{array}{c}\% \\
\text { Height }\end{array}$ \\
\hline 1 & 10.315 & 18508499 & 95.47 & 651200 & 97.22 \\
\hline 2 & 16.203 & 879094 & 4.53 & 18644 & 2.78 \\
\hline
\end{tabular}




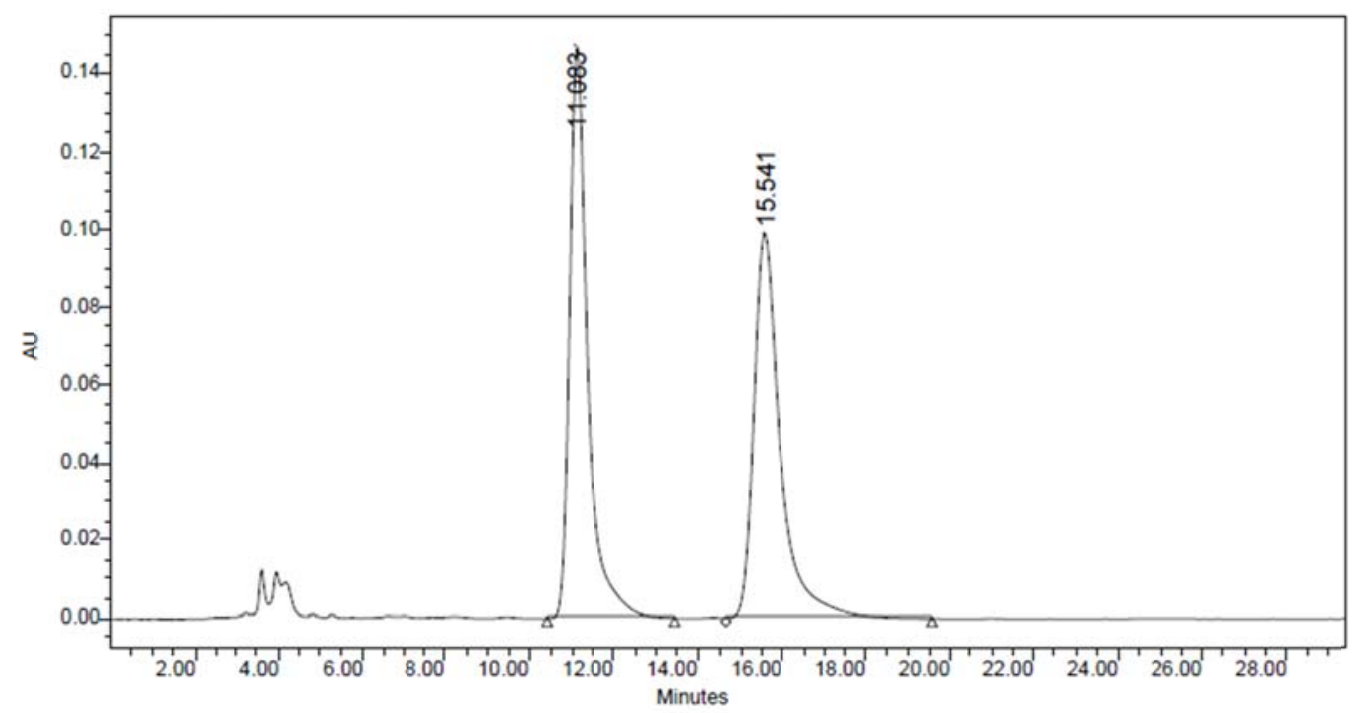

\begin{tabular}{|c|c|c|c|c|c|}
\hline & $\begin{array}{c}\text { RT } \\
(\mathrm{min})\end{array}$ & $\begin{array}{c}\text { Area } \\
\left(\mathrm{V}^{*} \mathrm{sec}\right)\end{array}$ & $\%$ Area & $\begin{array}{c}\text { Height } \\
(\mathrm{V})\end{array}$ & $\begin{array}{c}\% \\
\text { Height }\end{array}$ \\
\hline 1 & 11.083 & 4437690 & 50.37 & 147169 & 59.70 \\
\hline 2 & 15.541 & 4373363 & 49.63 & 99358 & 40.30 \\
\hline
\end{tabular}
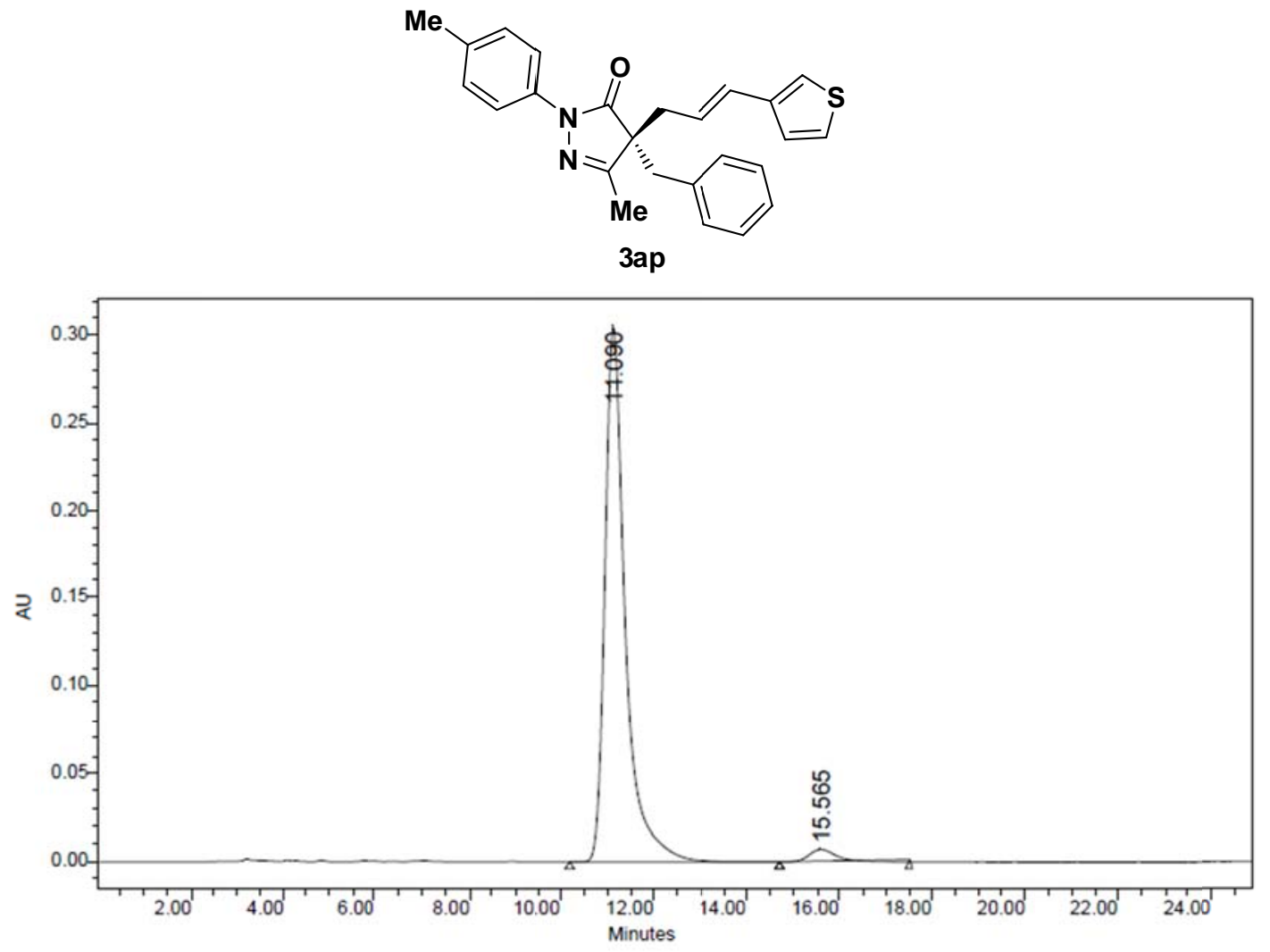

\begin{tabular}{|c|c|r|r|r|r|}
\hline & $\begin{array}{c}\text { RT } \\
(\mathrm{min})\end{array}$ & $\begin{array}{c}\text { Area } \\
\left(\mathrm{V}^{*} \mathrm{sec}\right)\end{array}$ & $\%$ Area & $\begin{array}{c}\text { Height } \\
(\mathrm{V})\end{array}$ & $\begin{array}{c}\% \\
\text { Height }\end{array}$ \\
\hline 1 & 11.090 & 9226810 & 96.83 & 305521 & 97.71 \\
\hline 2 & 15.565 & 302524 & 3.17 & 7164 & 2.29 \\
\hline
\end{tabular}




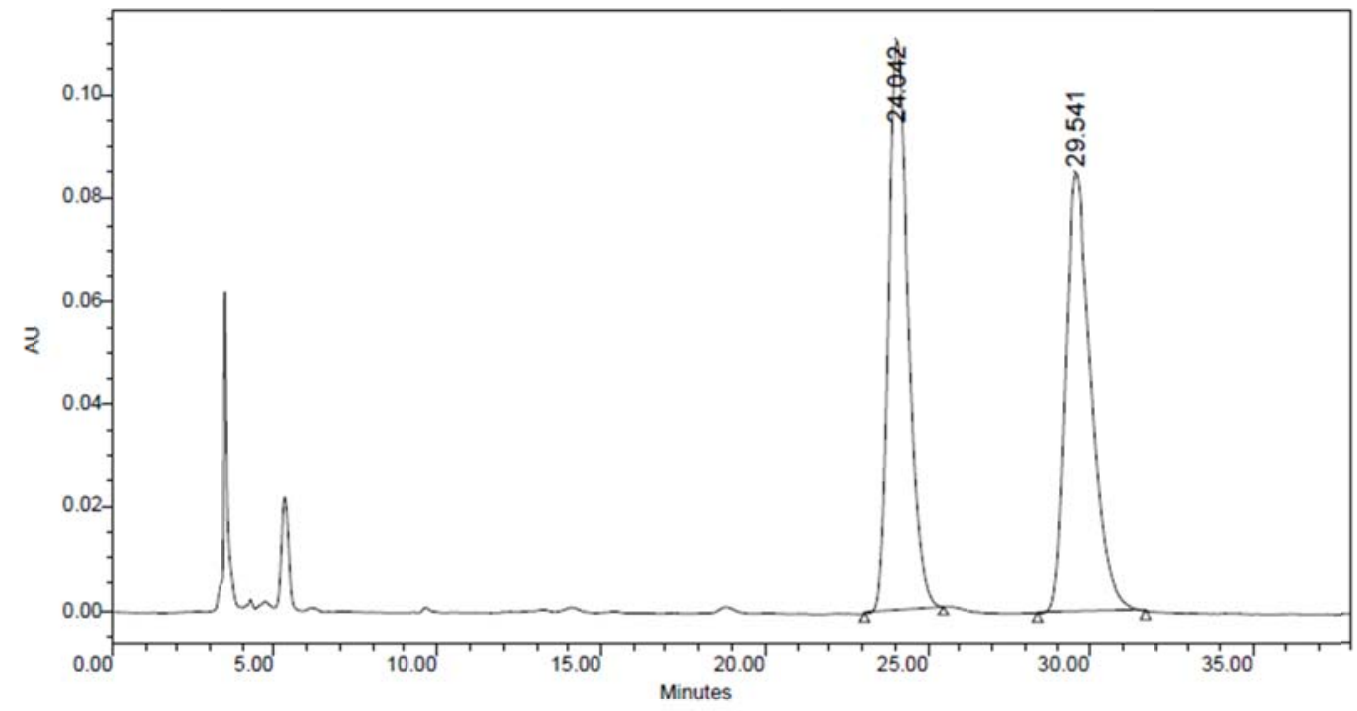

\begin{tabular}{|c|c|c|c|c|c|}
\hline & $\begin{array}{c}\text { RT } \\
(\mathrm{min})\end{array}$ & $\begin{array}{c}\text { Area } \\
\left(\mathrm{V}^{*} \mathrm{sec}\right)\end{array}$ & $\%$ Area & $\begin{array}{c}\text { Height } \\
(\mathrm{V})\end{array}$ & $\begin{array}{c}\% \\
\text { Height }\end{array}$ \\
\hline 1 & 24.042 & 4644712 & 50.21 & 110706 & 56.46 \\
\hline 2 & 29.541 & 4606392 & 49.79 & 85378 & 43.54 \\
\hline
\end{tabular}

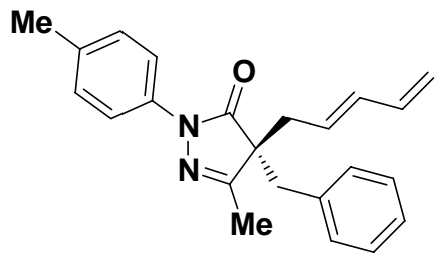

$5 a a$

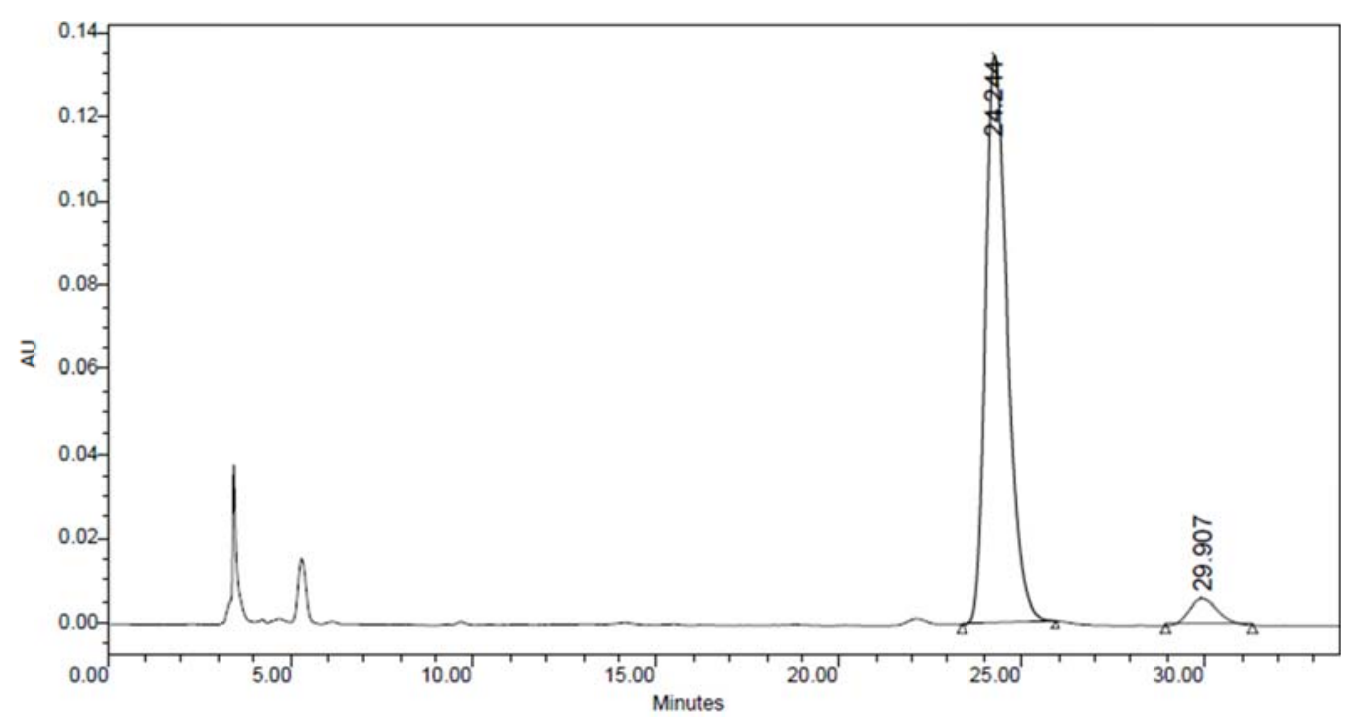

\begin{tabular}{|c|c|r|r|r|r|}
\hline & $\begin{array}{c}\text { RT } \\
(\mathrm{min})\end{array}$ & $\begin{array}{c}\text { Area } \\
\left(\mathrm{V}^{*} \mathrm{sec}\right)\end{array}$ & \% Area & $\begin{array}{c}\text { Height } \\
(\mathrm{V})\end{array}$ & $\begin{array}{c}\% \\
\text { Height }\end{array}$ \\
\hline 1 & 24.244 & 5573325 & 94.14 & 134563 & 95.55 \\
\hline 2 & 29.907 & 346667 & 5.86 & 6271 & 4.45 \\
\hline
\end{tabular}




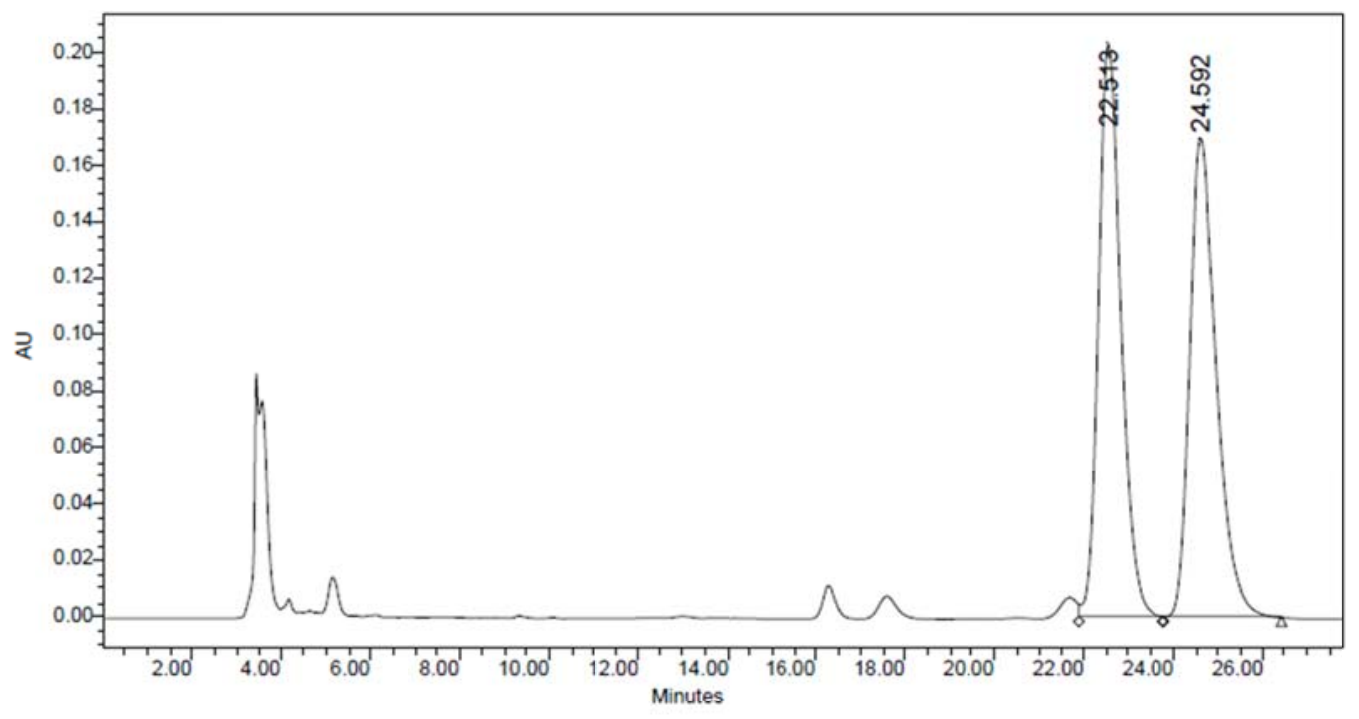

\begin{tabular}{|c|c|c|c|c|c|}
\hline & $\begin{array}{c}\text { RT } \\
(\mathrm{min})\end{array}$ & $\begin{array}{c}\text { Area } \\
\left(\mathrm{V}^{*} \mathrm{sec}\right)\end{array}$ & \% Area & $\begin{array}{c}\text { Height } \\
(\mathrm{V})\end{array}$ & $\begin{array}{c}\% \\
\text { Height }\end{array}$ \\
\hline 1 & 22.513 & 7127770 & 50.43 & 203292 & 54.45 \\
\hline 2 & 24.592 & 7006536 & 49.57 & 170071 & 45.55 \\
\hline
\end{tabular}

Me

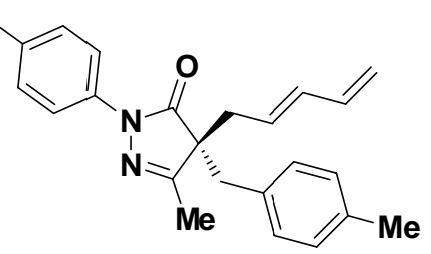

5 ba

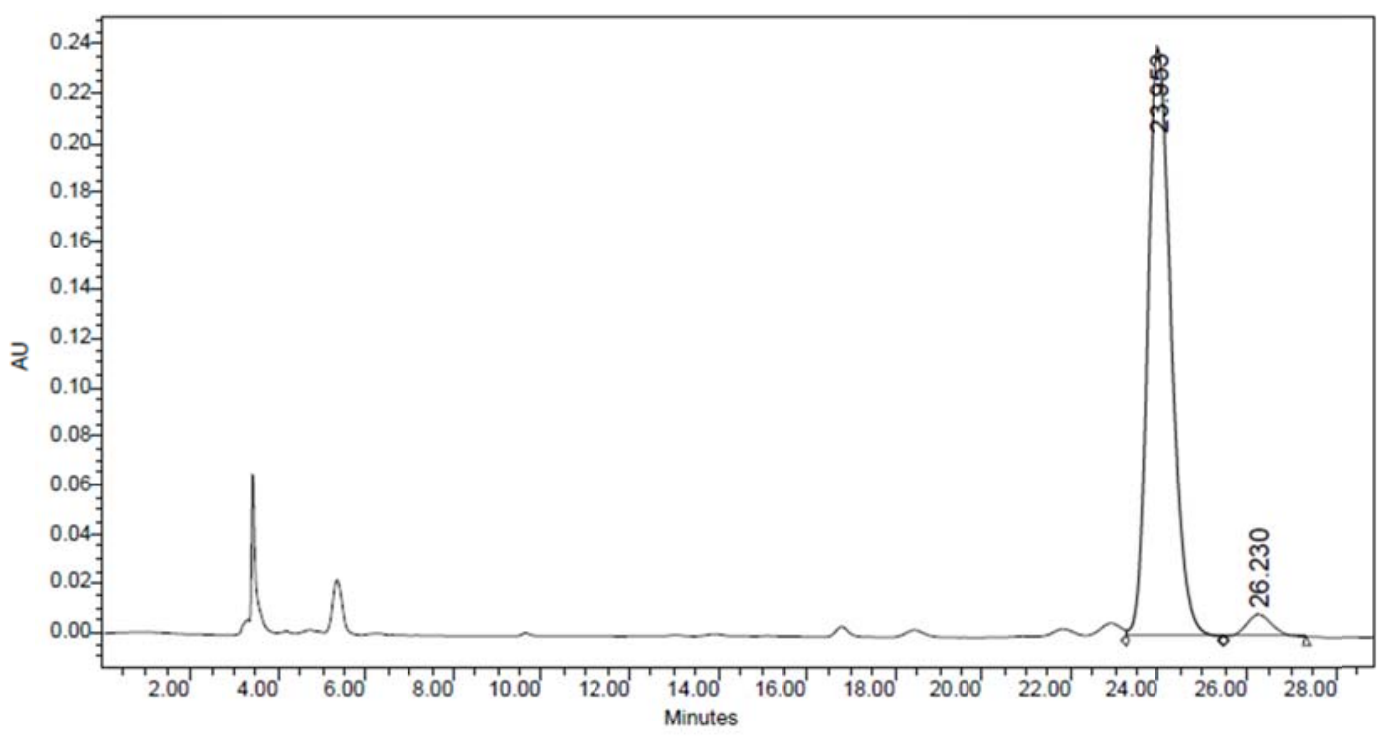

\begin{tabular}{|c|c|c|r|r|r|}
\hline & $\begin{array}{c}\text { RT } \\
(\mathrm{min})\end{array}$ & $\begin{array}{c}\text { Area } \\
\left(\mathrm{V}^{*} \mathrm{sec}\right)\end{array}$ & \% Area & $\begin{array}{c}\text { Height } \\
(\mathrm{V})\end{array}$ & $\begin{array}{c}\% \\
\text { Height }\end{array}$ \\
\hline 1 & 23.953 & 9083983 & 96.10 & 240262 & 96.36 \\
\hline 2 & 26.230 & 369025 & 3.90 & 9065 & 3.64 \\
\hline
\end{tabular}




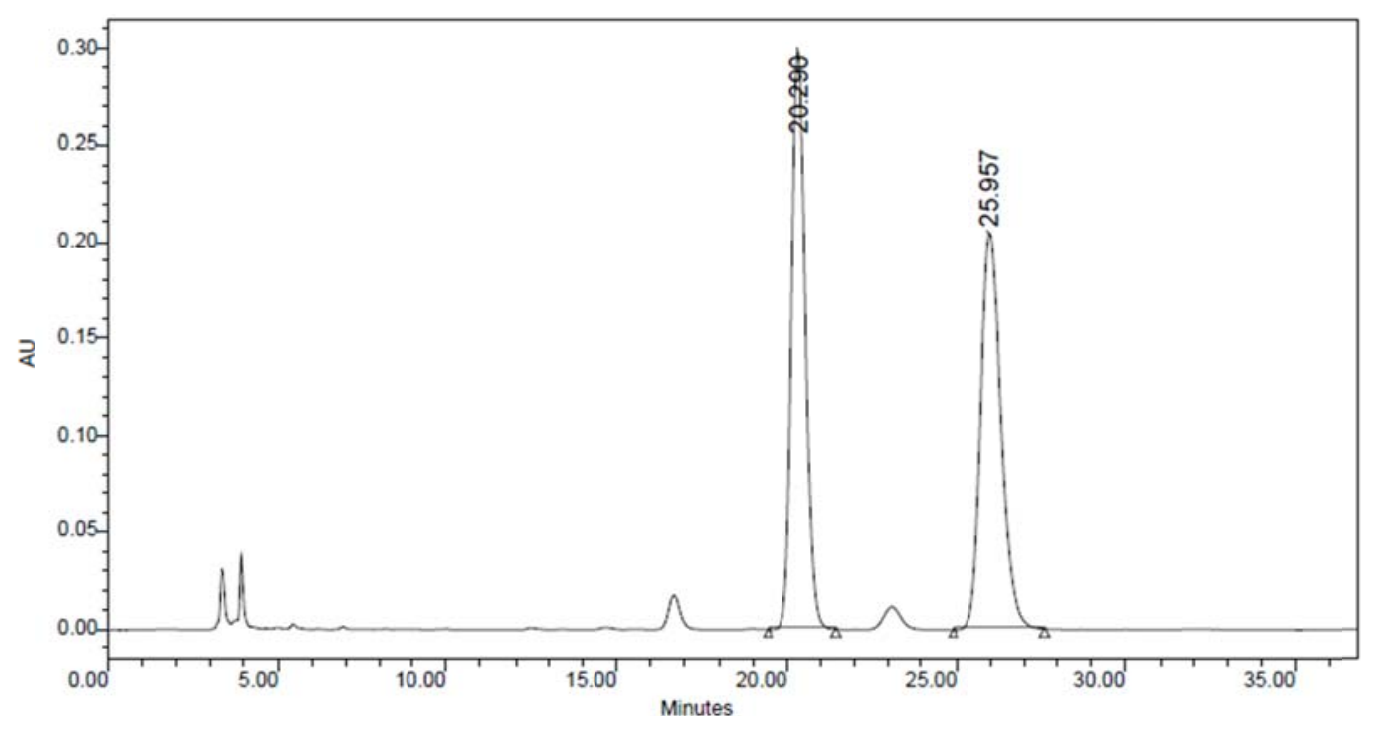

\begin{tabular}{|c|c|c|c|c|c|}
\hline & $\begin{array}{c}\text { RT } \\
(\mathrm{min})\end{array}$ & $\begin{array}{c}\text { Area } \\
\left(\mathrm{IV}^{*} \mathrm{sec}\right)\end{array}$ & $\%$ Area & $\begin{array}{c}\text { Height } \\
(\mathrm{V})\end{array}$ & $\begin{array}{c}\% \\
\text { Height }\end{array}$ \\
\hline 1 & 20.290 & 8617134 & 50.10 & 298928 & 59.34 \\
\hline 2 & 25.957 & 8584392 & 49.90 & 204845 & 40.66 \\
\hline
\end{tabular}
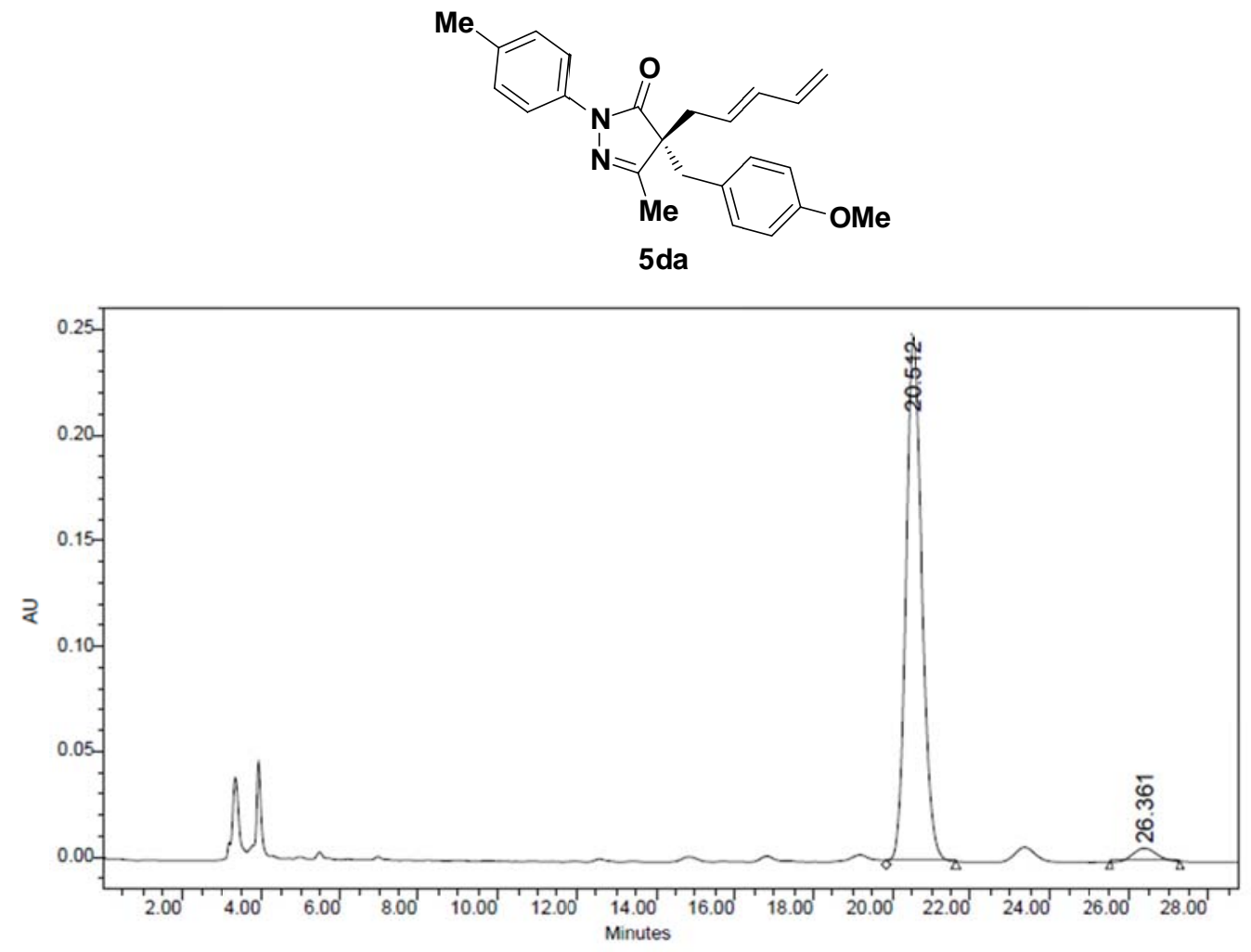

\begin{tabular}{|c|c|c|r|r|r|}
\hline & $\begin{array}{c}\text { RT } \\
(\mathrm{min})\end{array}$ & $\begin{array}{c}\text { Area } \\
\left(\mathrm{V}^{\prime} \mathrm{sec}\right)\end{array}$ & \% Area & $\begin{array}{c}\text { Height } \\
(\mathrm{V})\end{array}$ & $\begin{array}{c}\% \\
\text { Height }\end{array}$ \\
\hline 1 & 20.512 & 7263074 & 96.40 & 248706 & 97.47 \\
\hline 2 & 26.361 & 271058 & 3.60 & 6447 & 2.53 \\
\hline
\end{tabular}




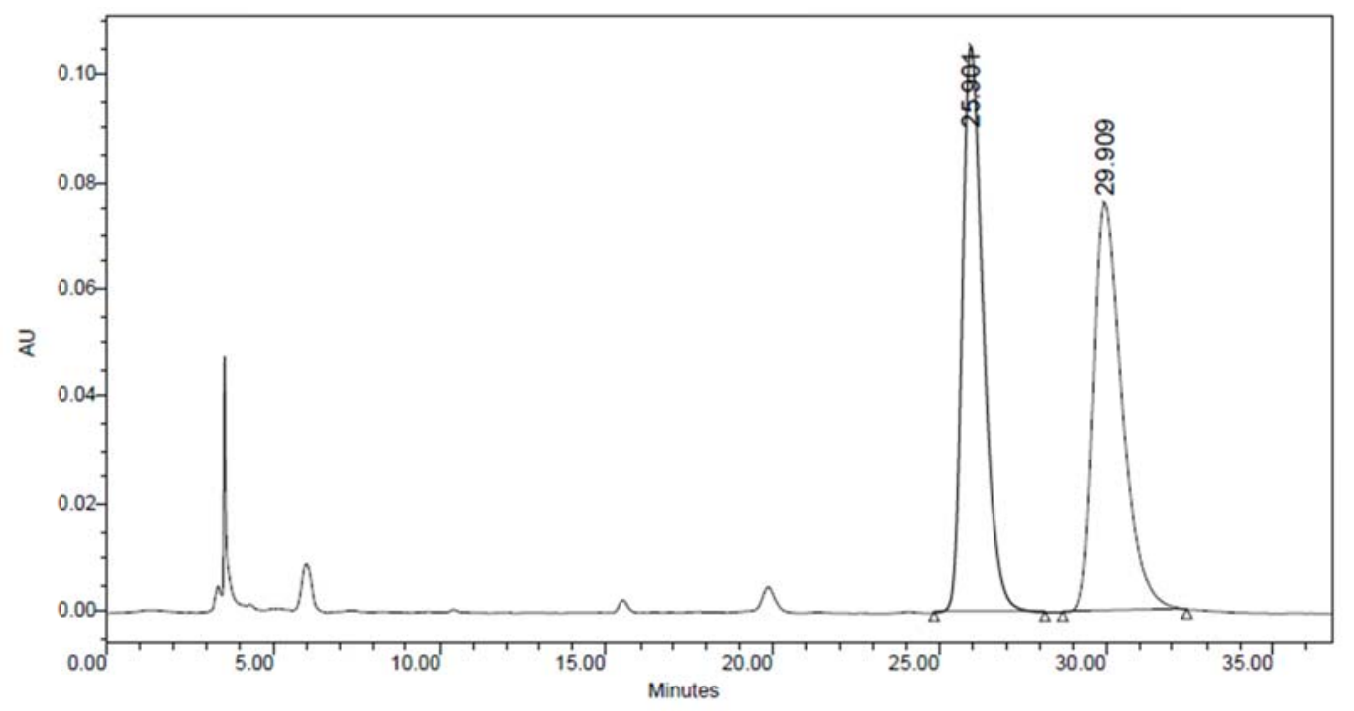

\begin{tabular}{|c|c|c|c|c|c|}
\hline & $\begin{array}{c}\text { RT } \\
(\mathrm{min})\end{array}$ & $\begin{array}{c}\text { Area } \\
\left(\mathrm{V}^{*} \mathrm{sec}\right)\end{array}$ & \% Area & $\begin{array}{c}\text { Height } \\
(\mathrm{V})\end{array}$ & $\begin{array}{c}\% \\
\text { Height }\end{array}$ \\
\hline 1 & 25.901 & 4656186 & 50.53 & 105493 & 58.09 \\
\hline 2 & 29.909 & 4558121 & 49.47 & 76105 & 41.91 \\
\hline
\end{tabular}

Me

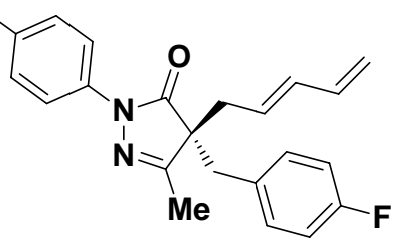

5 ea

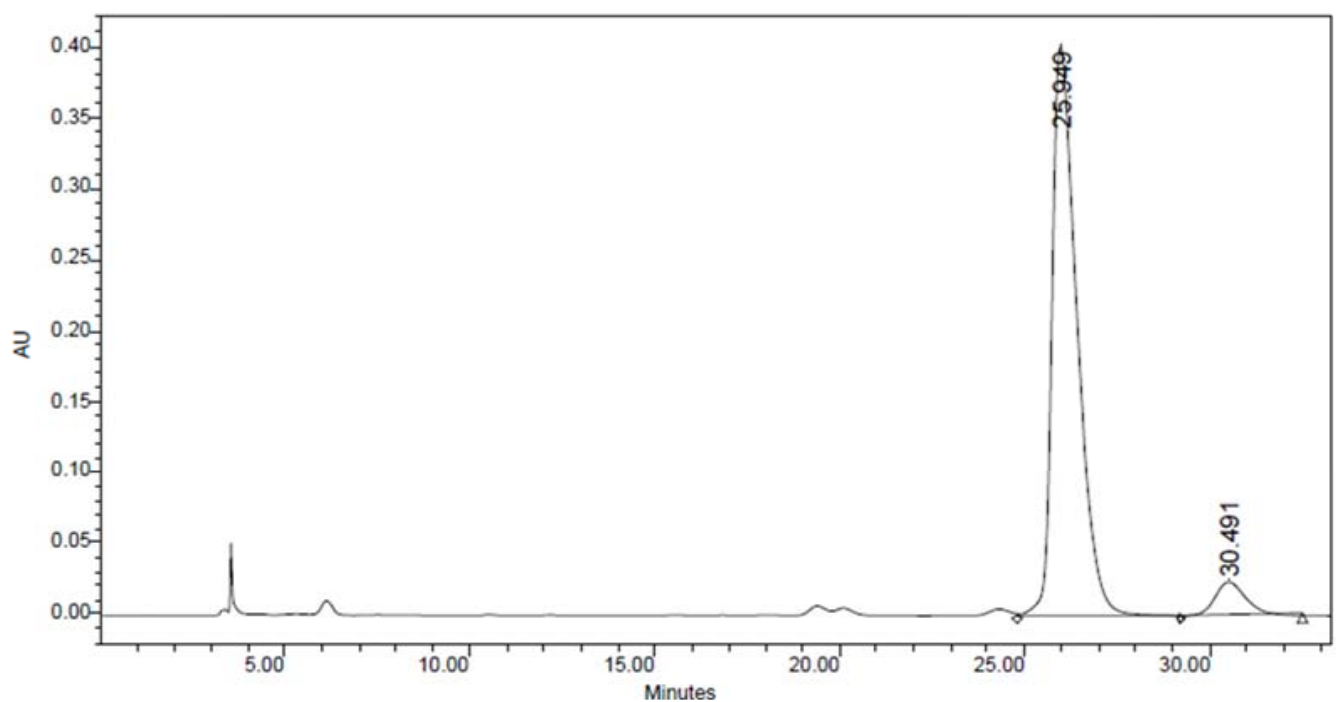

\begin{tabular}{|c|c|c|r|r|r|}
\hline & $\begin{array}{c}\mathrm{RT} \\
(\mathrm{min})\end{array}$ & $\begin{array}{c}\text { Area } \\
\left(\mathrm{V}^{*} \mathrm{sec}\right)\end{array}$ & $\%$ Area & $\begin{array}{c}\text { Height } \\
(\mathrm{V})\end{array}$ & $\begin{array}{c}\% \\
\text { Height }\end{array}$ \\
\hline 1 & 25.949 & 18658000 & 93.14 & 400573 & 94.43 \\
\hline 2 & 30.491 & 1373768 & 6.86 & 23629 & 5.57 \\
\hline
\end{tabular}




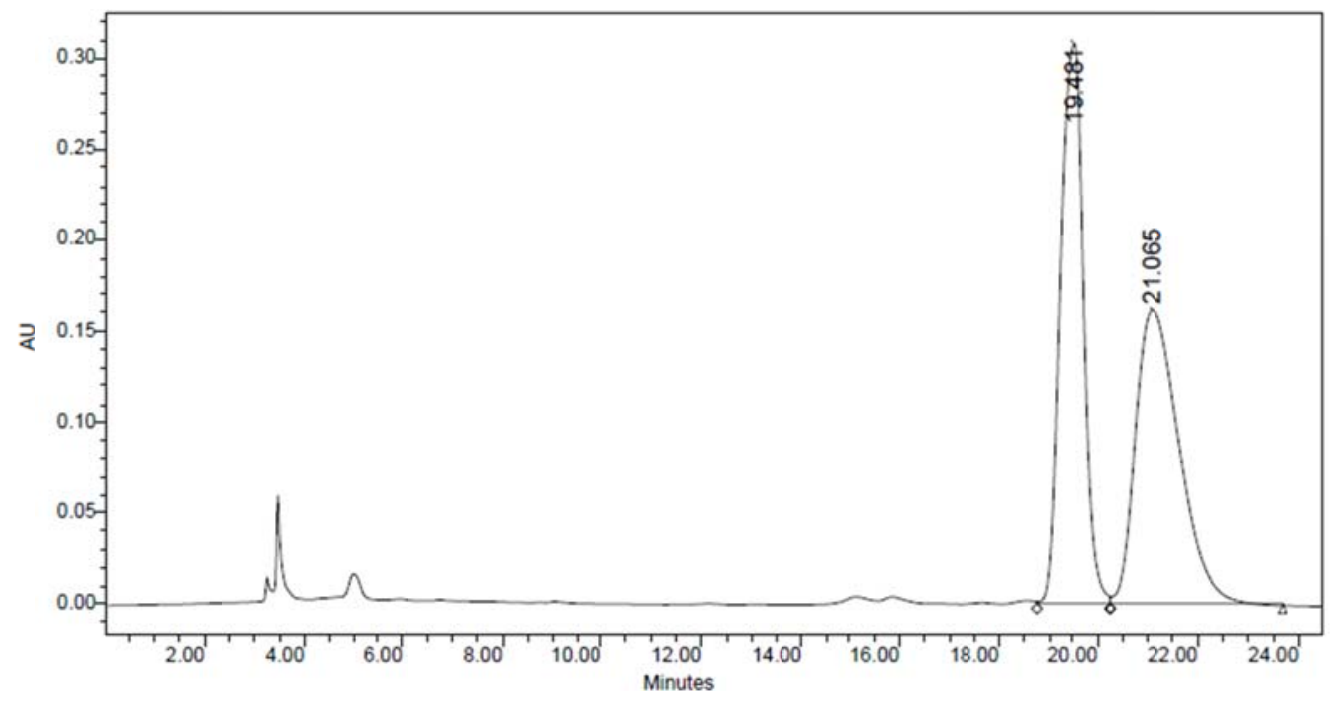

\begin{tabular}{|c|c|c|c|c|c|}
\hline & $\begin{array}{c}\text { RT } \\
(\mathrm{min})\end{array}$ & $\begin{array}{c}\text { Area } \\
\left(\mathrm{V}^{*} \mathrm{sec}\right)\end{array}$ & \% Area & $\begin{array}{c}\text { Height } \\
(\mathrm{V})\end{array}$ & $\begin{array}{c}\% \\
\text { Height }\end{array}$ \\
\hline 1 & 19.481 & 9845221 & 49.70 & 308720 & 65.61 \\
\hline 2 & 21.065 & 9964809 & 50.30 & 161797 & 34.39 \\
\hline
\end{tabular}
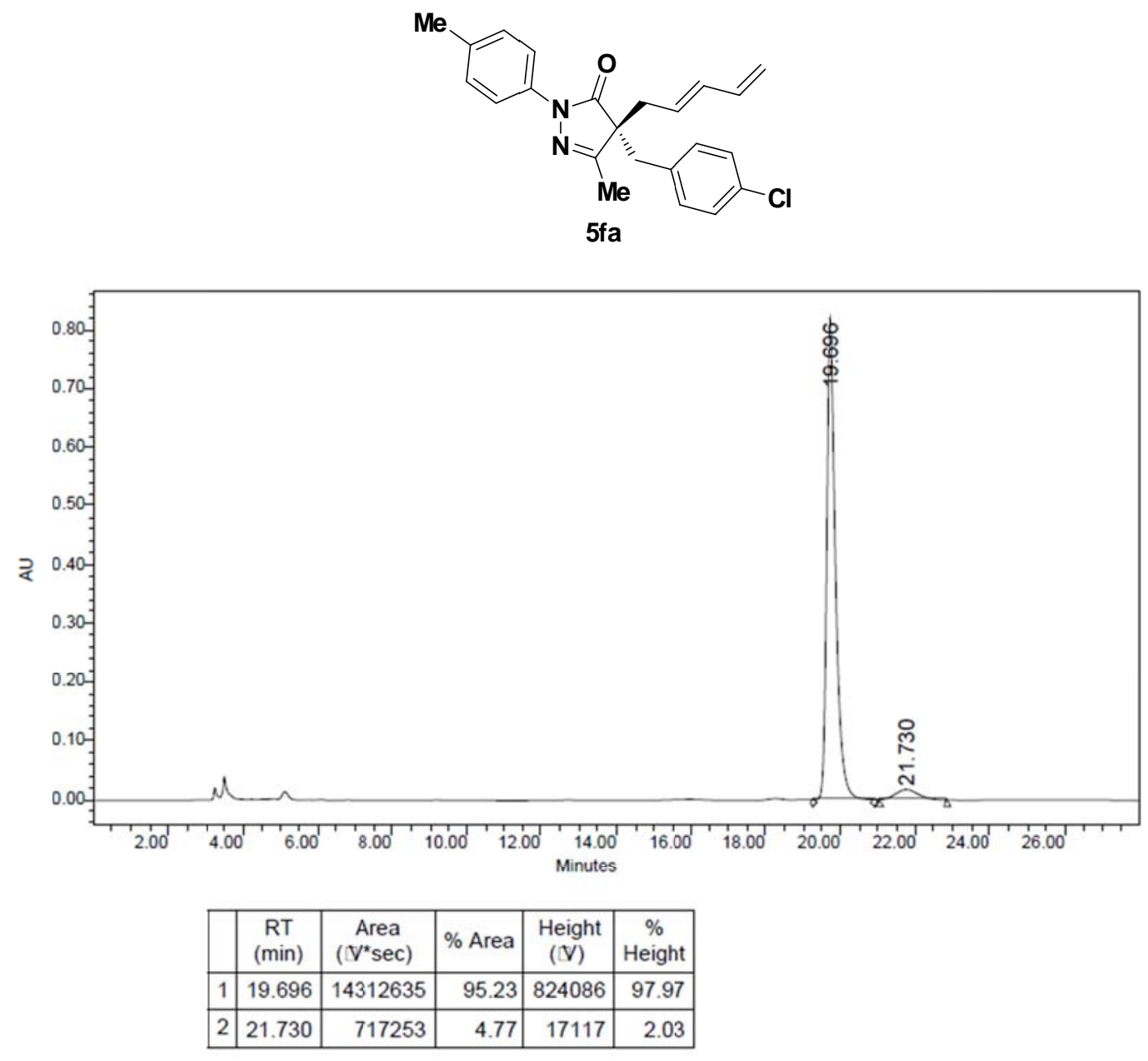


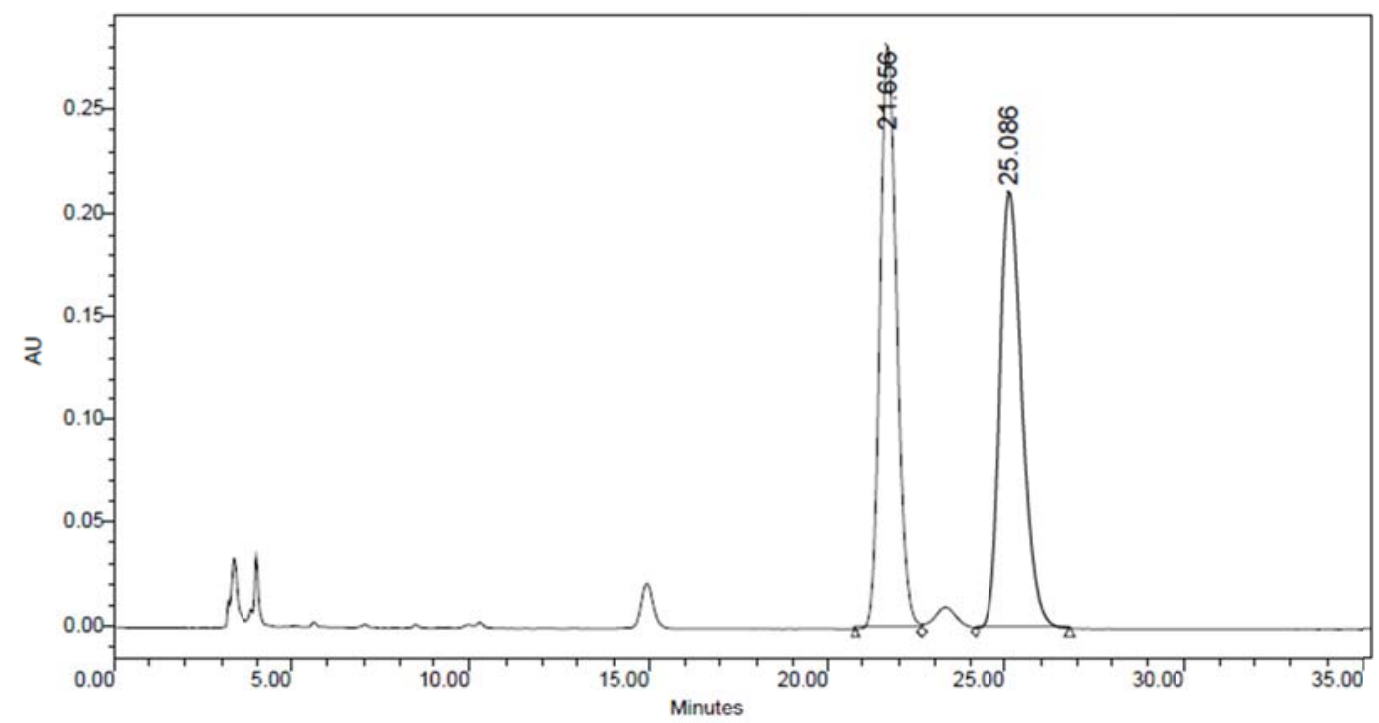

\begin{tabular}{|c|c|c|c|c|c|}
\hline & $\begin{array}{c}\text { RT } \\
(\mathrm{min})\end{array}$ & $\begin{array}{c}\text { Area } \\
\left(\mathrm{V}^{*} \mathrm{sec}\right)\end{array}$ & $\%$ Area & $\begin{array}{c}\text { Height } \\
(\mathrm{V})\end{array}$ & $\begin{array}{c}\% \\
\text { Height }\end{array}$ \\
\hline 1 & 21.656 & 9261804 & 50.06 & 281922 & 57.13 \\
\hline 2 & 25.086 & 9239472 & 49.94 & 211510 & 42.87 \\
\hline
\end{tabular}

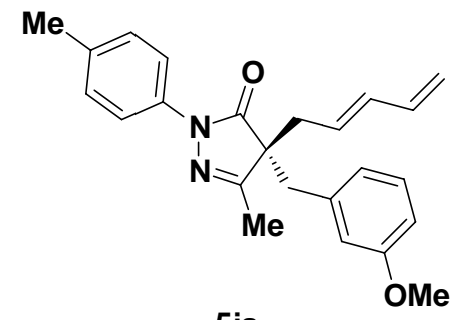

$5 i a$

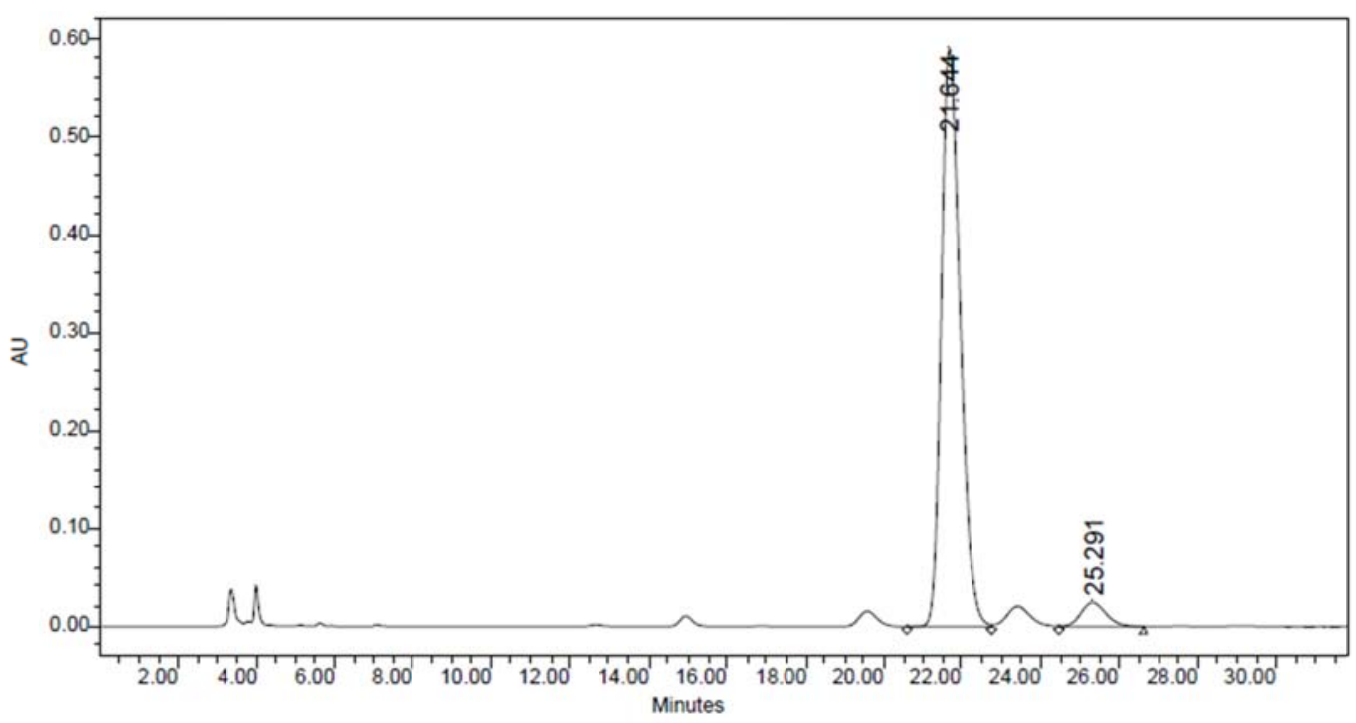

\begin{tabular}{|c|c|c|r|r|r|}
\hline & $\begin{array}{c}\text { RT } \\
(\mathrm{min})\end{array}$ & $\begin{array}{c}\text { Area } \\
\left(\mathrm{V}^{*} \mathrm{sec}\right)\end{array}$ & \% Area & $\begin{array}{c}\text { Height } \\
(\mathrm{V})\end{array}$ & $\begin{array}{c}\% \\
\text { Height }\end{array}$ \\
\hline 1 & 21.644 & 19929261 & 94.77 & 591289 & 95.99 \\
\hline 2 & 25.291 & 1099513 & 5.23 & 24676 & 4.01 \\
\hline
\end{tabular}




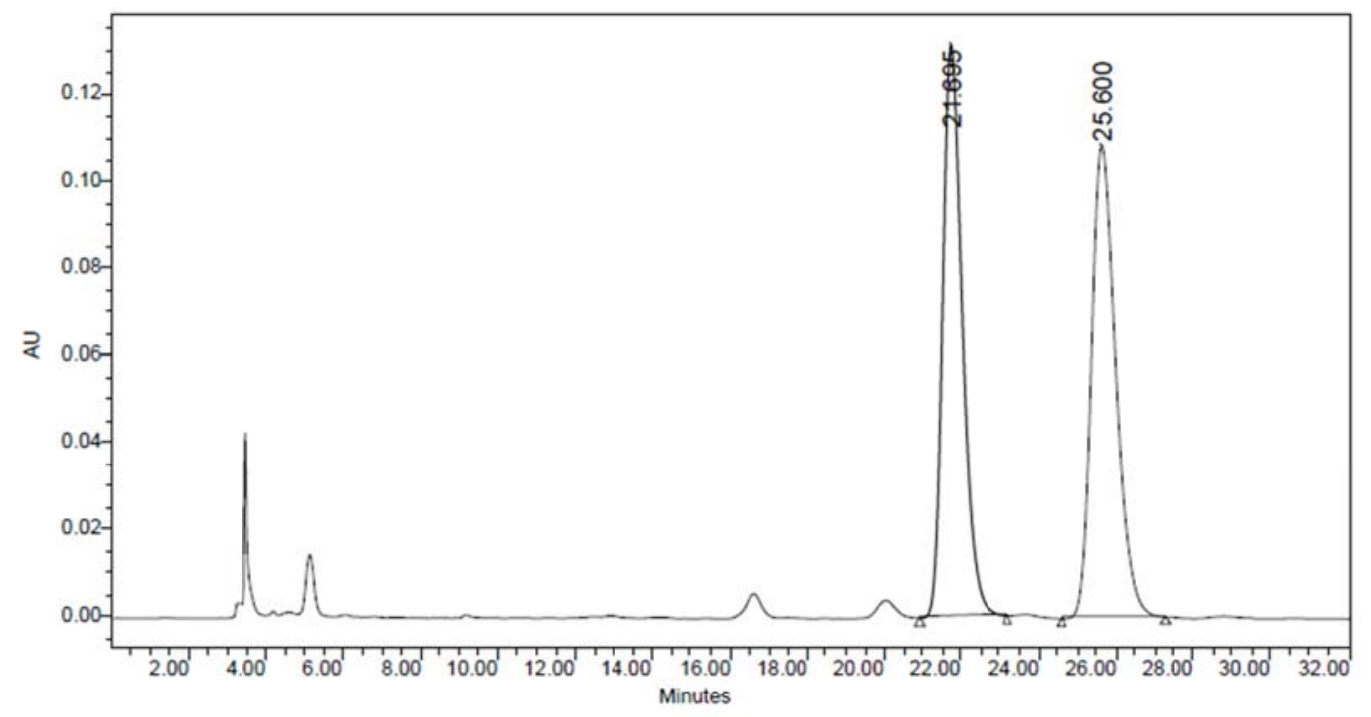

\begin{tabular}{|c|c|c|c|c|c|}
\hline & $\begin{array}{c}\text { RT } \\
(\mathrm{min})\end{array}$ & $\begin{array}{c}\text { Area } \\
\left(\mathrm{V}^{*} \mathrm{sec}\right)\end{array}$ & \% Area & $\begin{array}{c}\text { Height } \\
(\mathrm{V})\end{array}$ & $\begin{array}{c}\% \\
\text { Height }\end{array}$ \\
\hline 1 & 21.695 & 4653623 & 49.68 & 131418 & 54.76 \\
\hline 2 & 25.600 & 4713231 & 50.32 & 108587 & 45.24 \\
\hline
\end{tabular}
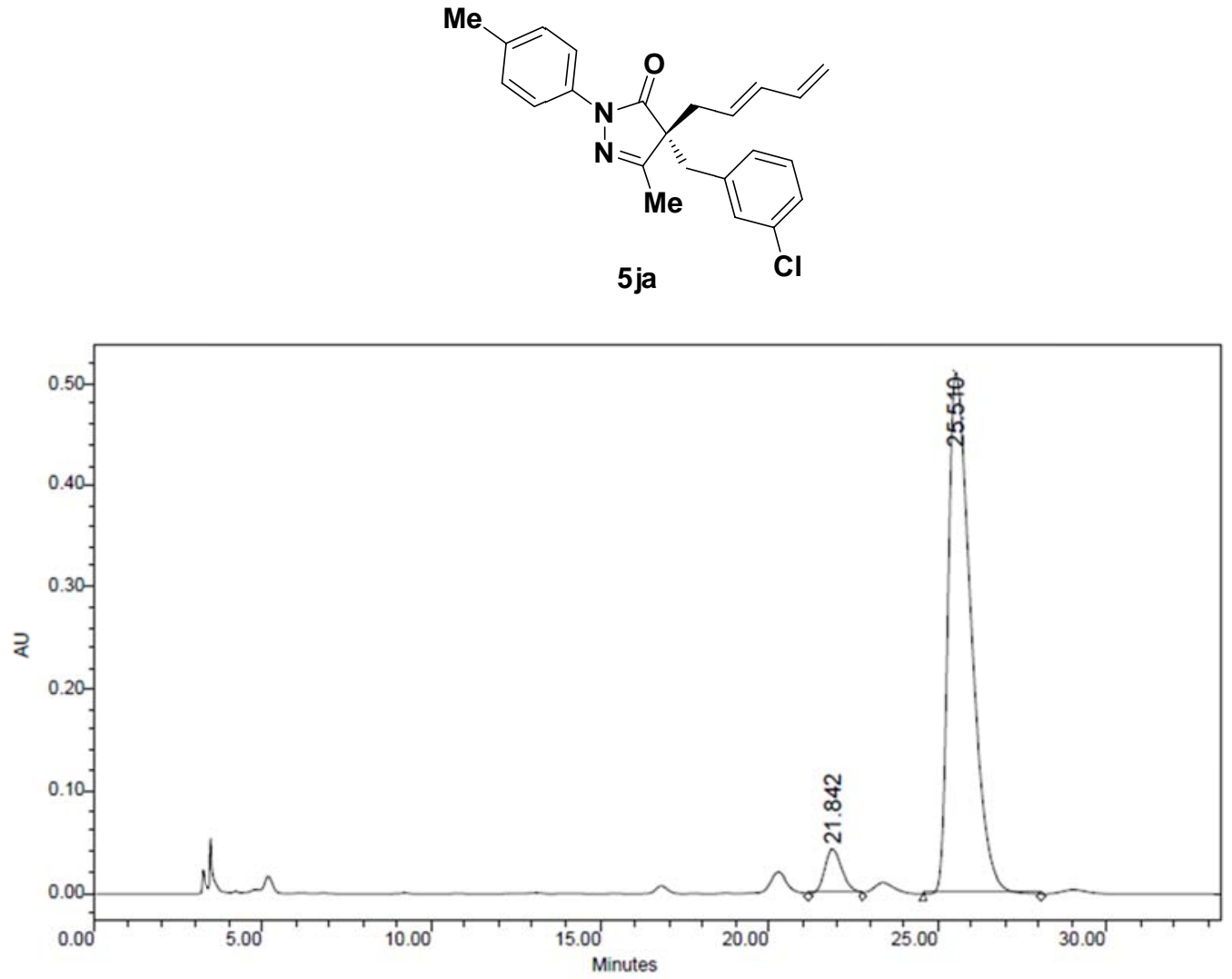

\begin{tabular}{|c|c|c|r|r|r|}
\hline & $\begin{array}{c}\text { RT } \\
(\mathrm{min})\end{array}$ & $\begin{array}{c}\text { Area } \\
\left(\mathrm{V}^{*} \mathrm{sec}\right)\end{array}$ & $\%$ Area & $\begin{array}{c}\text { Height } \\
(\mathrm{V})\end{array}$ & $\begin{array}{c}\% \\
\text { Height }\end{array}$ \\
\hline 1 & 21.842 & 1549716 & 6.12 & 42831 & 7.72 \\
\hline 2 & 25.510 & 23753859 & 93.88 & 512097 & 92.28 \\
\hline
\end{tabular}




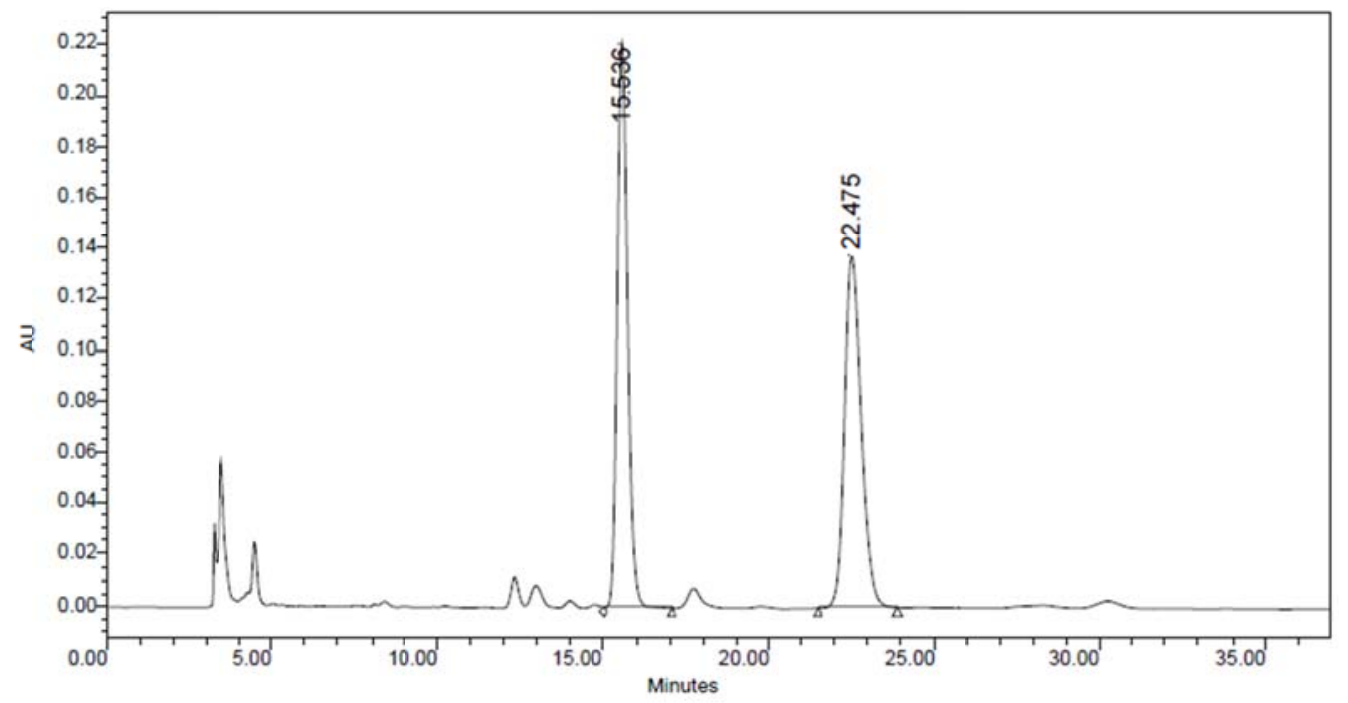

\begin{tabular}{|c|c|c|c|c|c|}
\hline & $\begin{array}{c}\text { RT } \\
(\mathrm{min})\end{array}$ & $\begin{array}{c}\text { Area } \\
\left(\mathrm{V}^{*} \mathrm{sec}\right)\end{array}$ & $\%$ Area & $\begin{array}{c}\text { Height } \\
(\mathrm{V})\end{array}$ & $\begin{array}{c}\% \\
\text { Height }\end{array}$ \\
\hline 1 & 15.536 & 4803501 & 49.87 & 221515 & 61.66 \\
\hline 2 & 22.475 & 4827870 & 50.13 & 137718 & 38.34 \\
\hline
\end{tabular}

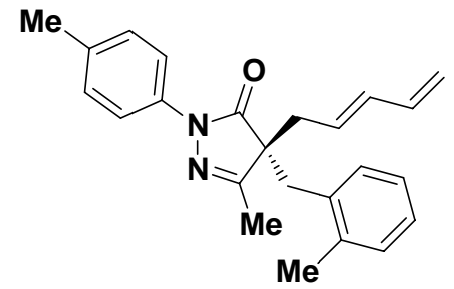

5ra

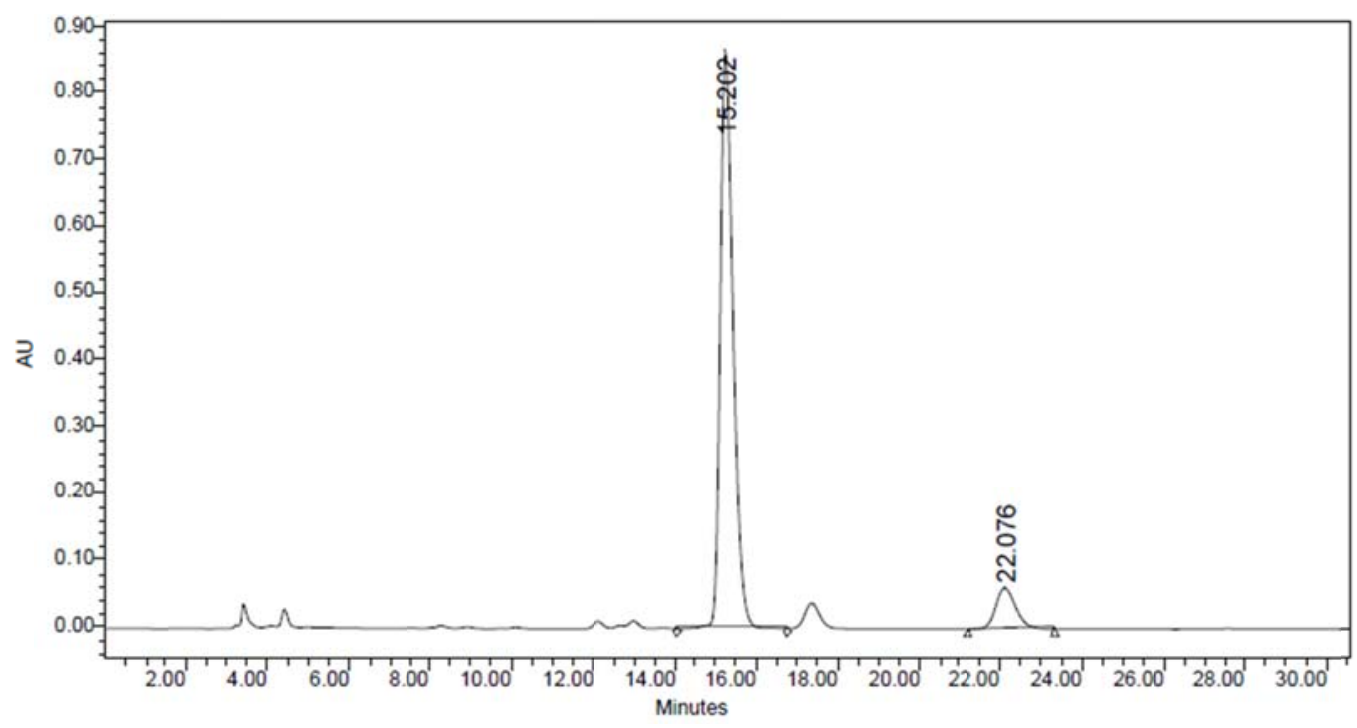

\begin{tabular}{|c|c|c|r|r|r|}
\hline & $\begin{array}{c}\mathrm{RT} \\
(\mathrm{min})\end{array}$ & $\begin{array}{c}\text { Area } \\
\left(\mathrm{V}^{*} \mathrm{sec}\right)\end{array}$ & $\%$ Area & $\begin{array}{c}\text { Height } \\
(\mathrm{V})\end{array}$ & $\begin{array}{c}\% \\
\text { Height }\end{array}$ \\
\hline 1 & 15.202 & 18963440 & 90.21 & 862674 & 93.42 \\
\hline 2 & 22.076 & 2058088 & 9.79 & 60731 & 6.58 \\
\hline
\end{tabular}




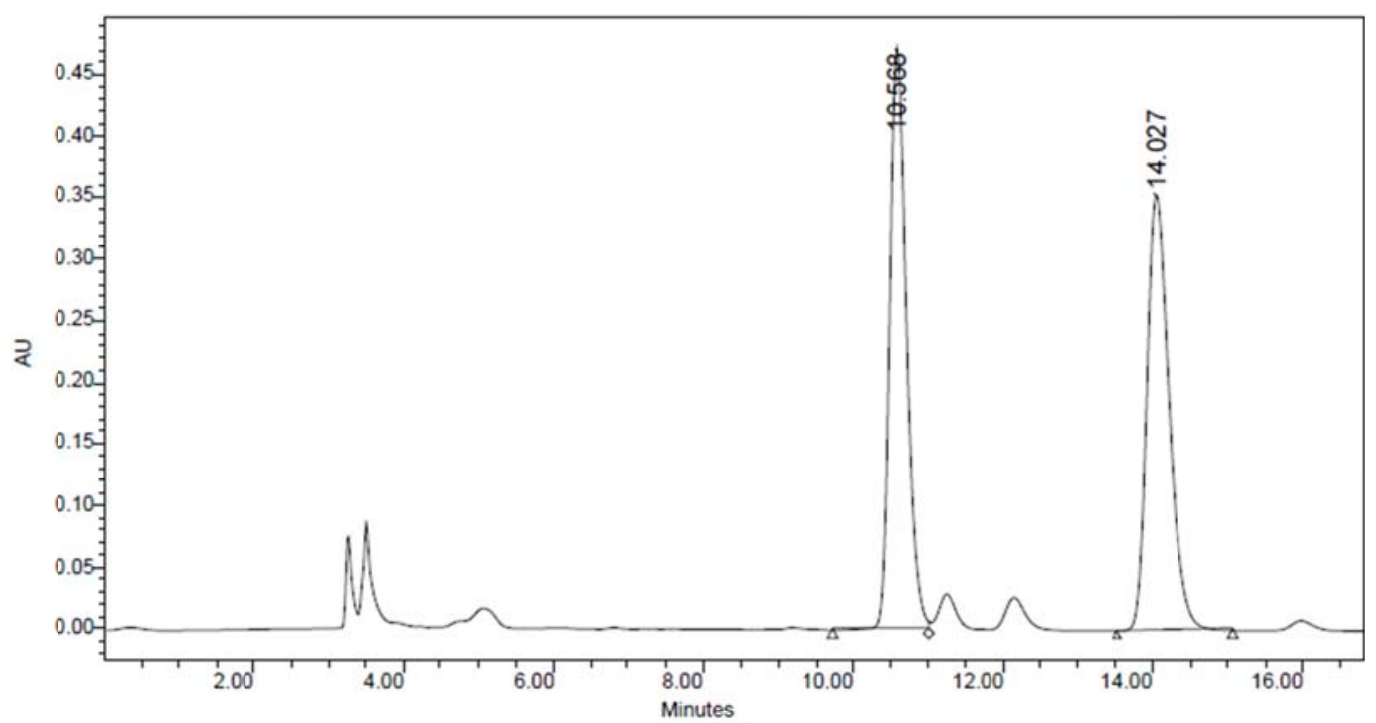

\begin{tabular}{|c|c|c|c|c|c|}
\hline & $\begin{array}{c}\text { RT } \\
(\mathrm{min})\end{array}$ & $\begin{array}{c}\text { Area } \\
\left(\mathrm{V}^{*} \mathrm{sec}\right)\end{array}$ & \% Area & $\begin{array}{c}\text { Height } \\
(\mathrm{V})\end{array}$ & $\begin{array}{c}\% \\
\text { Height }\end{array}$ \\
\hline 1 & 10.568 & 7317354 & 50.11 & 472429 & 57.18 \\
\hline 2 & 14.027 & 7286452 & 49.89 & 353728 & 42.82 \\
\hline
\end{tabular}

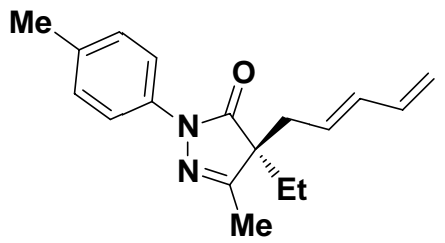

$50 a$

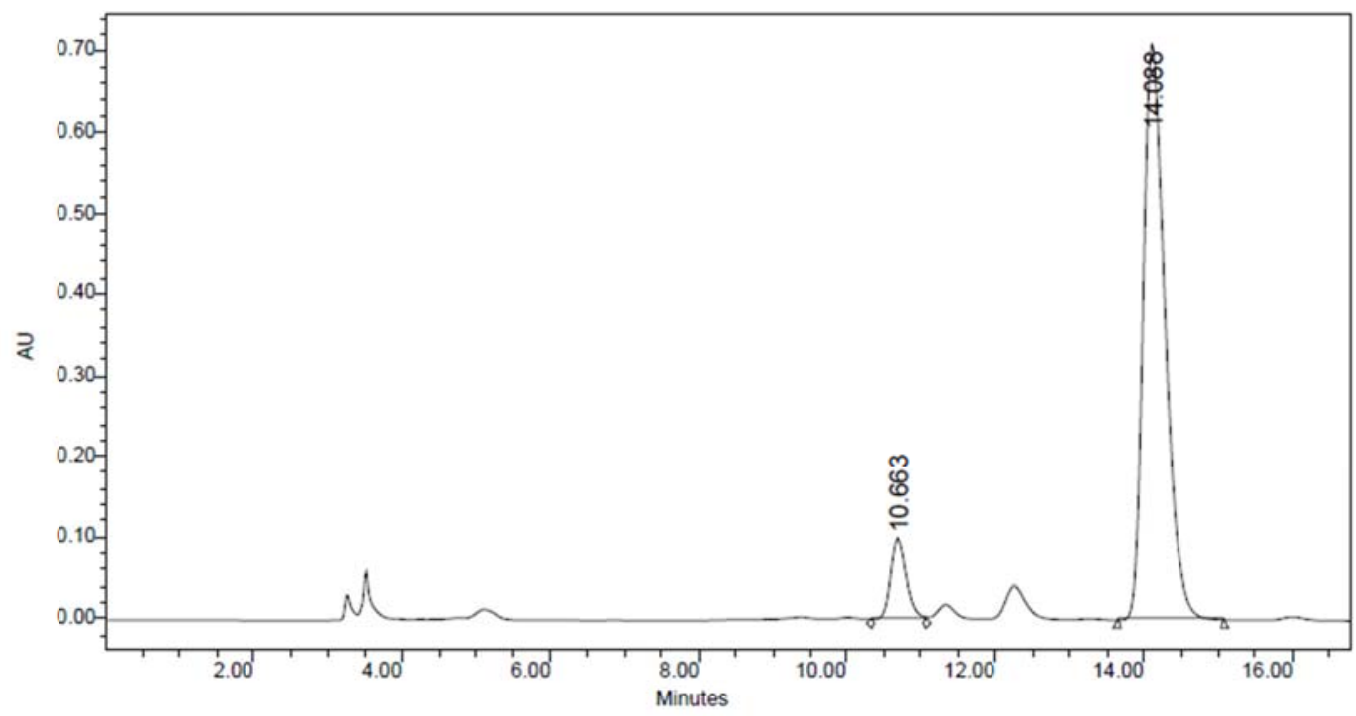

\begin{tabular}{|c|c|c|r|r|c|}
\hline & $\begin{array}{c}\text { RT } \\
(\mathrm{min})\end{array}$ & $\begin{array}{c}\text { Area } \\
\left(\mathrm{V}^{*} \mathrm{sec}\right)\end{array}$ & $\%$ Area & $\begin{array}{c}\text { Height } \\
(\mathrm{V})\end{array}$ & $\begin{array}{c}\% \\
\text { Height }\end{array}$ \\
\hline 1 & 10.663 & 1517222 & 9.46 & 99118 & 12.24 \\
\hline 2 & 14.088 & 14513701 & 90.54 & 710701 & 87.76 \\
\hline
\end{tabular}




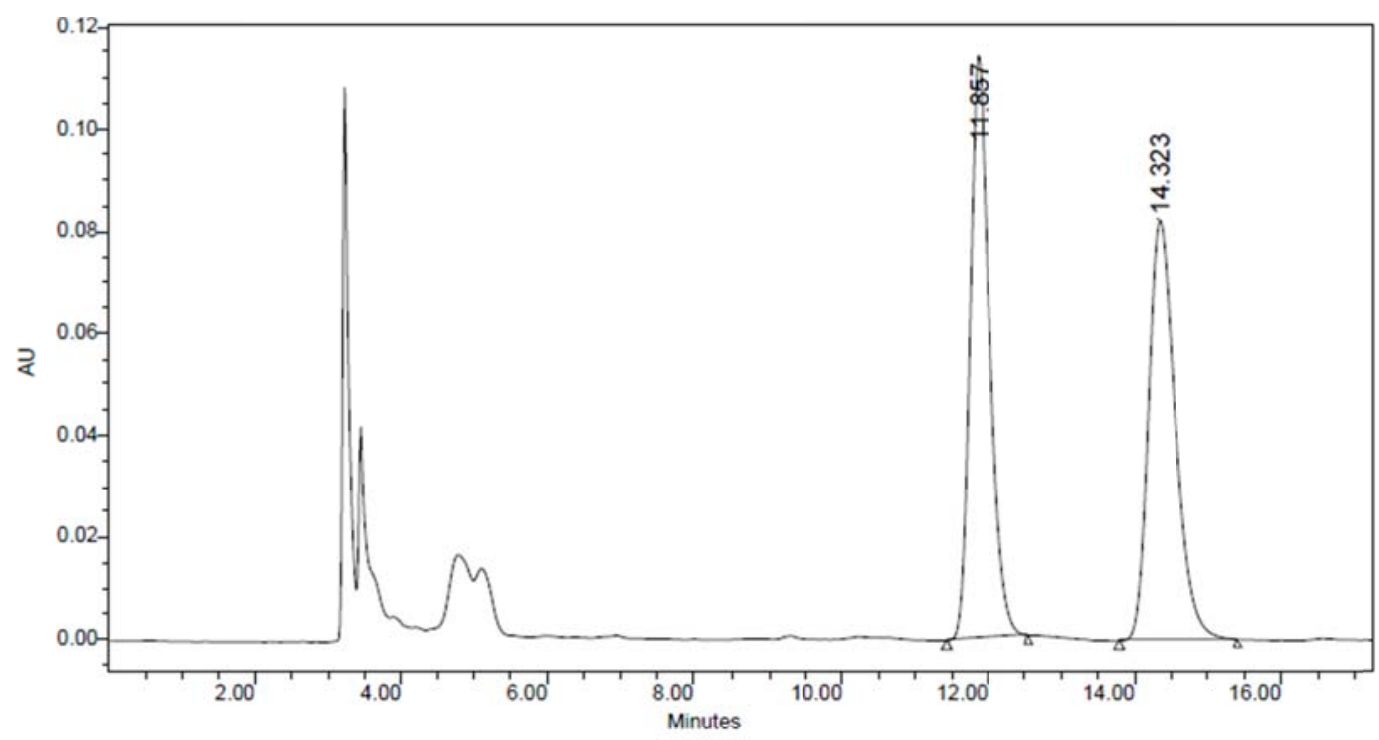

\begin{tabular}{|c|c|c|c|c|c|}
\hline & $\begin{array}{c}\text { RT } \\
(\mathrm{min})\end{array}$ & $\begin{array}{c}\text { Area } \\
\left(\mathrm{V}^{*} \mathrm{sec}\right)\end{array}$ & \% Area & $\begin{array}{c}\text { Height } \\
(\mathrm{V})\end{array}$ & $\begin{array}{c}\% \\
\text { Height }\end{array}$ \\
\hline 1 & 11.857 & 2115488 & 50.23 & 114282 & 58.11 \\
\hline 2 & 14.323 & 2095772 & 49.77 & 82385 & 41.89 \\
\hline
\end{tabular}

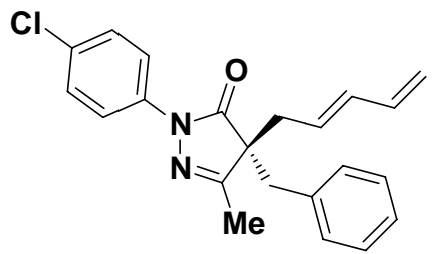

$5 q a$

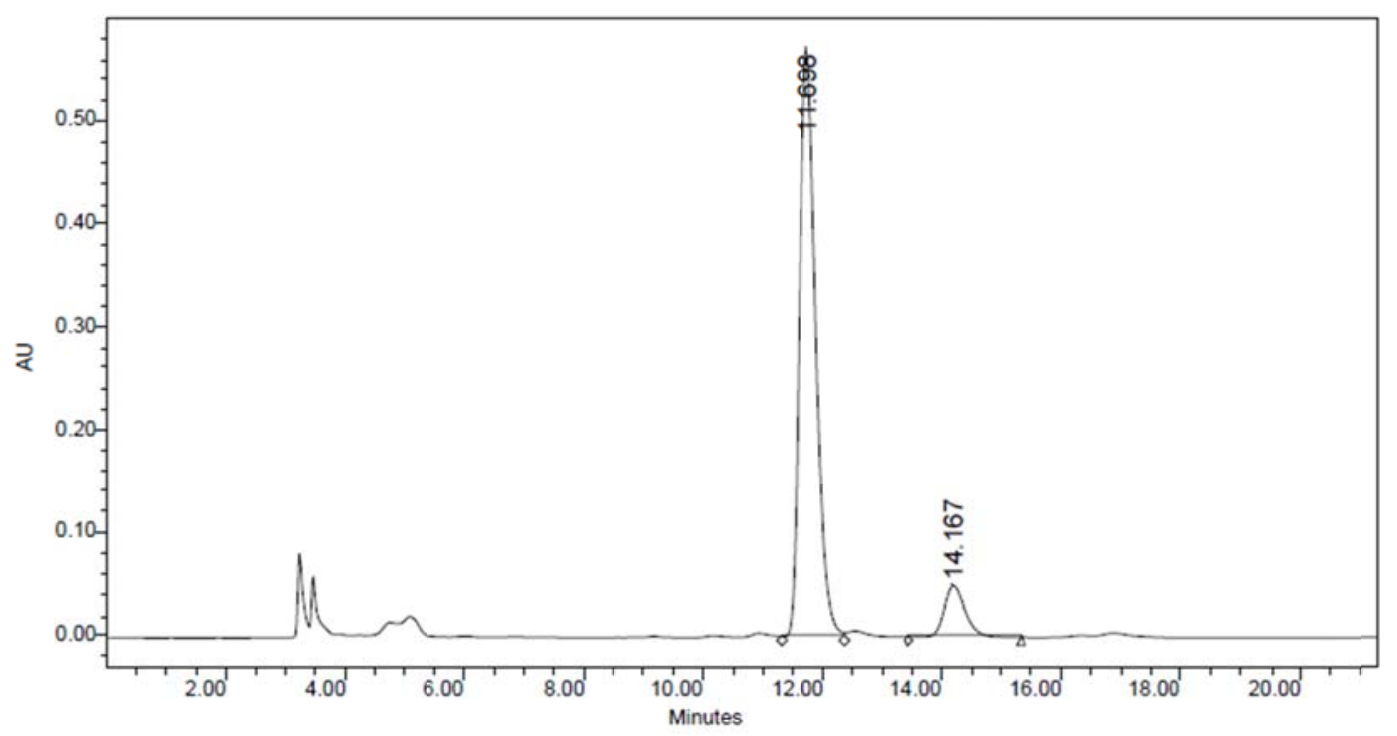

\begin{tabular}{|c|c|c|r|r|r|}
\hline & $\begin{array}{c}\text { RT } \\
(\mathrm{min})\end{array}$ & $\begin{array}{c}\text { Area } \\
\left(\mathrm{V}^{*} \mathrm{sec}\right)\end{array}$ & $\%$ Area & $\begin{array}{c}\text { Height } \\
(\mathrm{V})\end{array}$ & $\begin{array}{c}\% \\
\text { Height }\end{array}$ \\
\hline 1 & 11.698 & 10857771 & 89.90 & 571049 & 92.03 \\
\hline 2 & 14.167 & 1220263 & 10.10 & 49485 & 7.97 \\
\hline
\end{tabular}




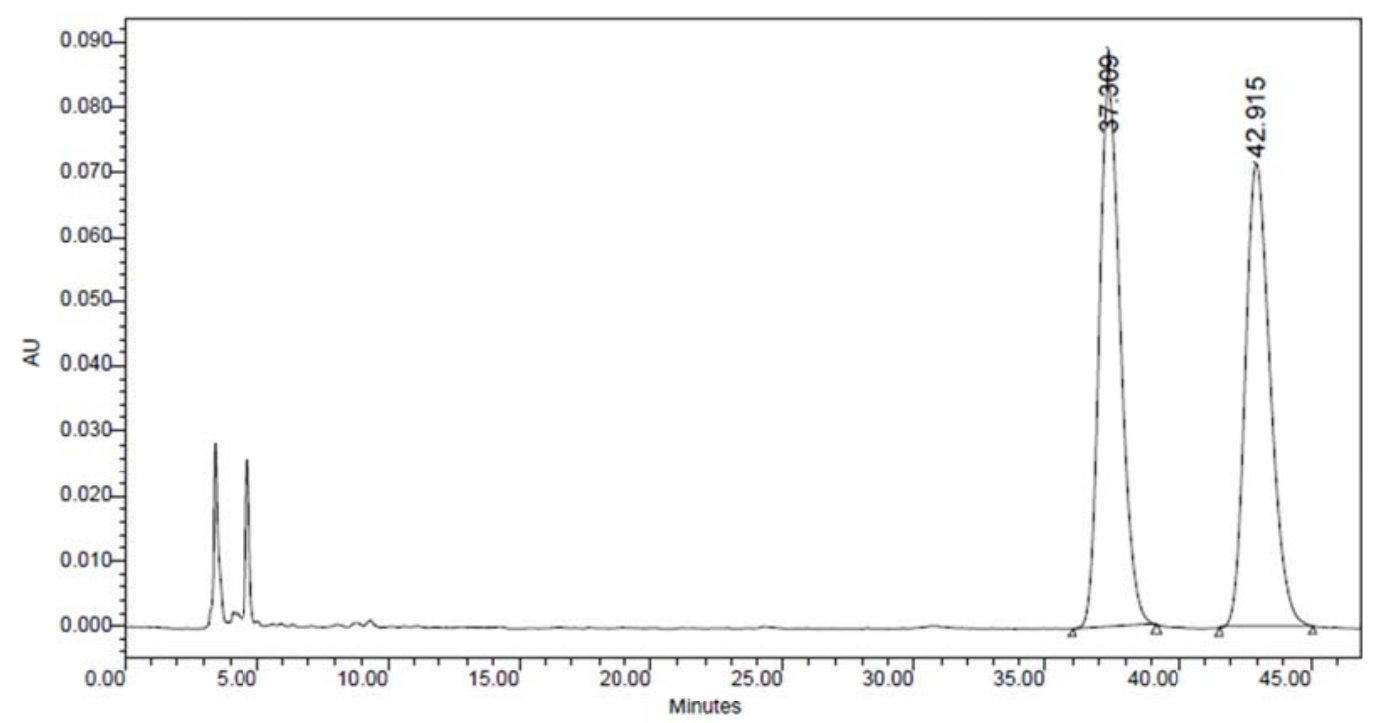

\begin{tabular}{|c|c|c|c|c|c|}
\hline & $\begin{array}{c}\text { RT } \\
(\mathrm{min})\end{array}$ & $\begin{array}{c}\text { Area } \\
\left(\mathrm{V}^{*} \mathrm{sec}\right)\end{array}$ & $\%$ Area & $\begin{array}{c}\text { Height } \\
(\mathrm{V})\end{array}$ & $\begin{array}{c}\% \\
\text { Height }\end{array}$ \\
\hline 1 & 37.309 & 4738929 & 50.30 & 89167 & 55.44 \\
\hline 2 & 42.915 & 4682029 & 49.70 & 71658 & 44.56 \\
\hline
\end{tabular}

MeO

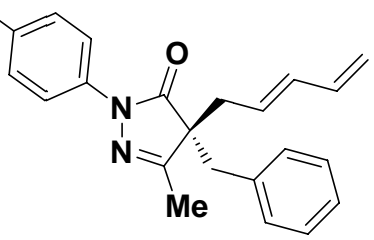

5sa

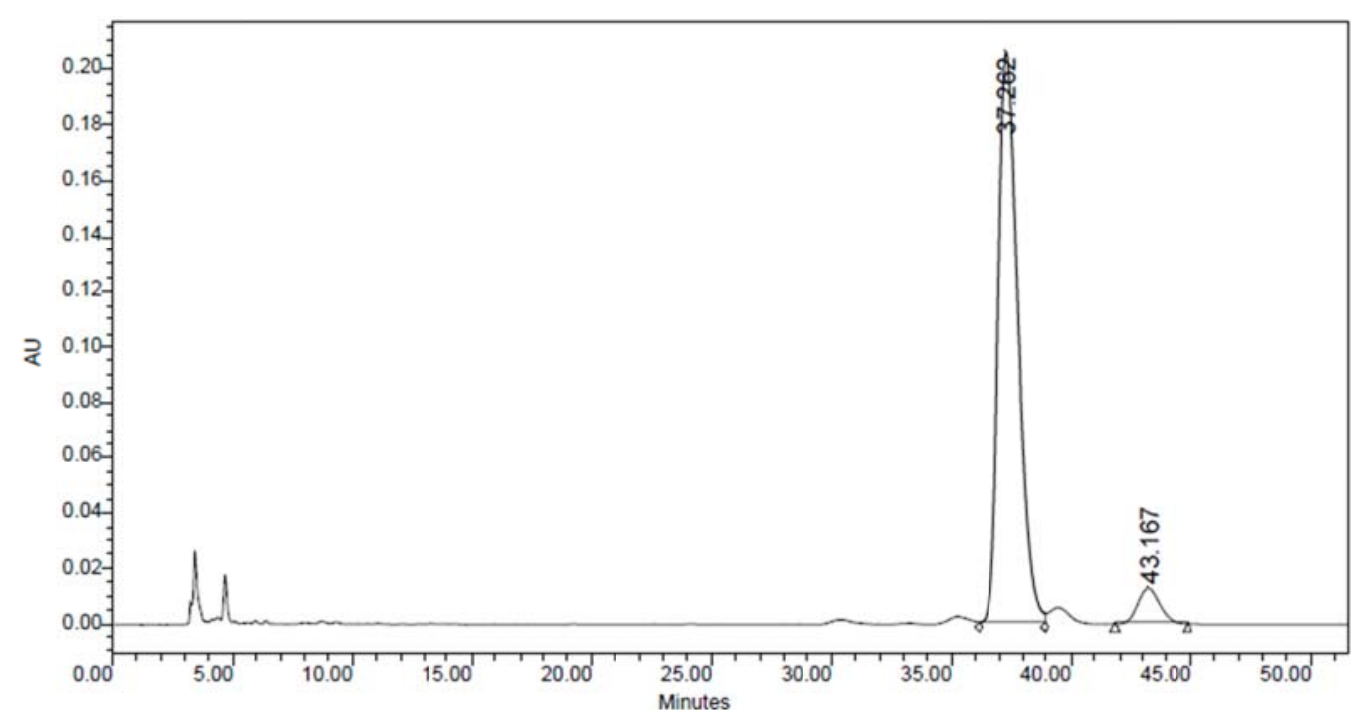

\begin{tabular}{|l|c|r|r|r|r|}
\hline & $\begin{array}{c}\text { RT } \\
(\mathrm{min})\end{array}$ & $\begin{array}{c}\text { Area } \\
\left(\mathrm{V}^{*} \mathrm{sec}\right)\end{array}$ & \% Area & $\begin{array}{c}\text { Height } \\
(\mathrm{V})\end{array}$ & $\begin{array}{c}\text { \% } \\
\text { Height }\end{array}$ \\
\hline 1 & 37.262 & 11943578 & 93.47 & 206302 & 94.12 \\
\hline 2 & 43.167 & 834643 & 6.53 & 12881 & 5.88 \\
\hline
\end{tabular}




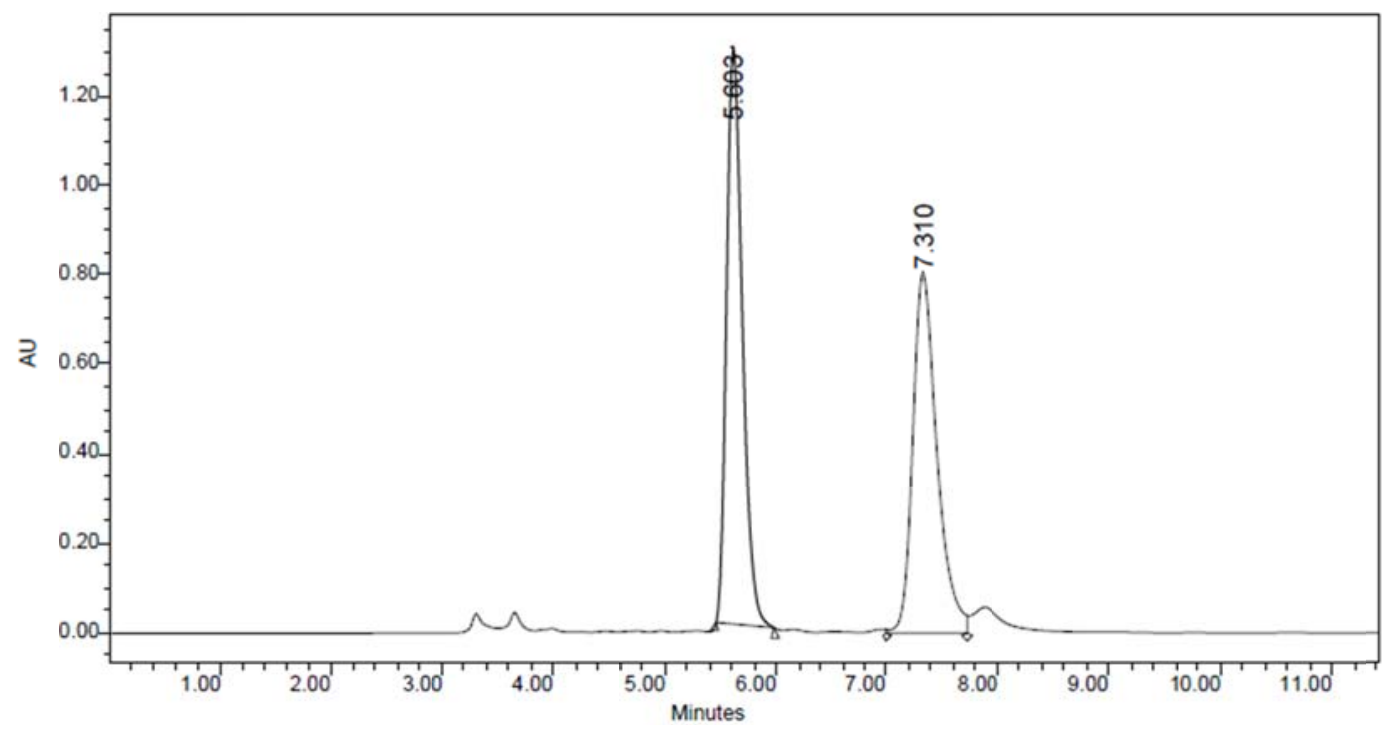

\begin{tabular}{|c|c|c|c|c|c|}
\hline & $\begin{array}{c}\text { RT } \\
(\mathrm{min})\end{array}$ & $\begin{array}{c}\text { Area } \\
\left(\mathrm{V}^{*} \mathrm{sec}\right)\end{array}$ & $\%$ Area & $\begin{array}{c}\text { Height } \\
(\mathrm{V})\end{array}$ & $\begin{array}{c}\% \\
\text { Height }\end{array}$ \\
\hline 1 & 5.603 & 12771019 & 51.15 & 1299218 & 61.66 \\
\hline 2 & 7.310 & 12195740 & 48.85 & 807749 & 38.34 \\
\hline
\end{tabular}
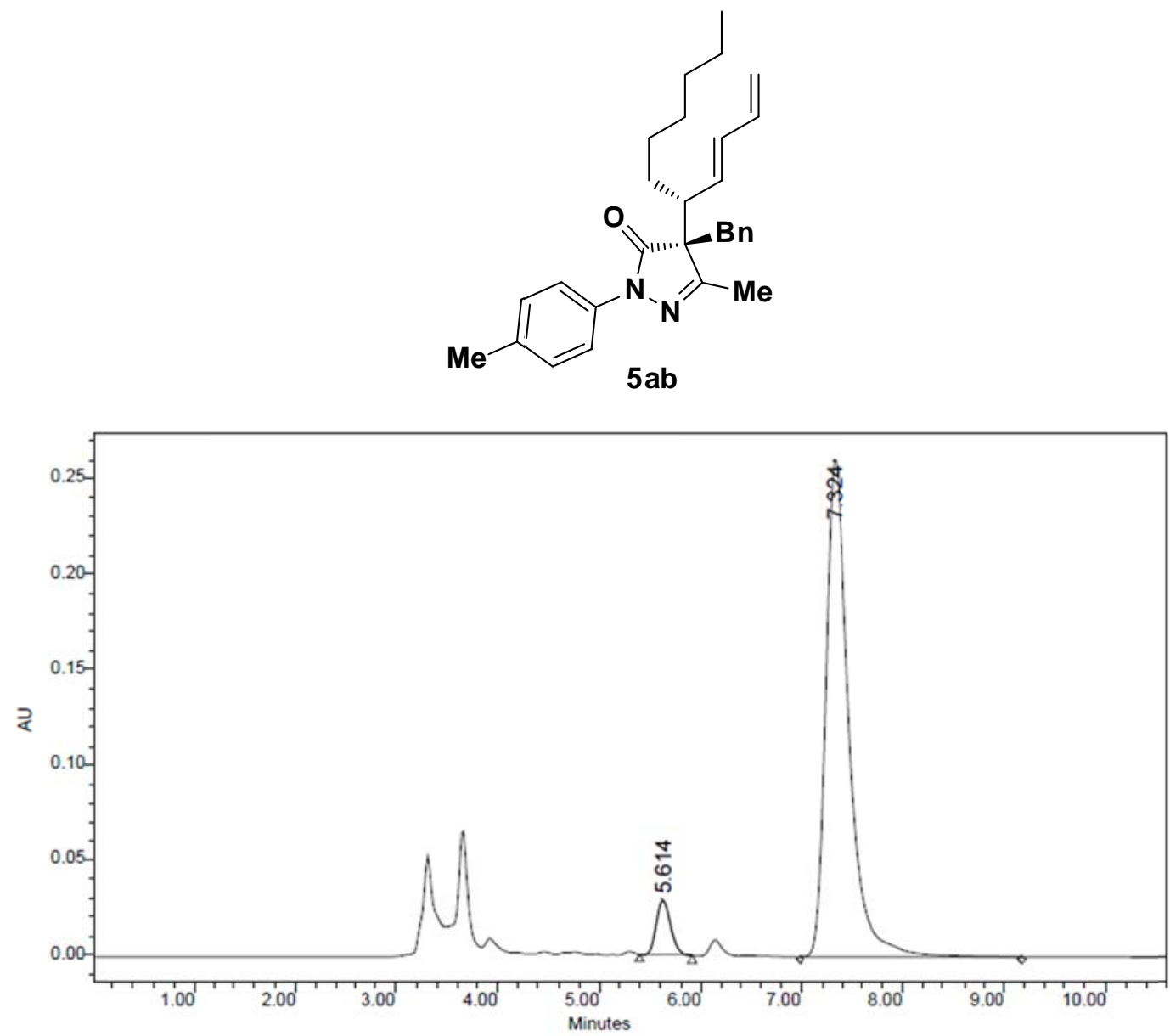

\begin{tabular}{|r|c|r|r|r|r|}
\hline & $\begin{array}{c}\text { RT } \\
(\mathrm{min})\end{array}$ & $\begin{array}{c}\text { Area } \\
\left(\mathrm{V}^{*} \mathrm{sec}\right)\end{array}$ & \% Area & $\begin{array}{l}\text { Height } \\
(\mathrm{V})\end{array}$ & $\begin{array}{c}\% \\
\text { Height }\end{array}$ \\
\hline 1 & 5.614 & 280151 & 6.27 & 28522 & 9.84 \\
\hline 2 & 7.324 & 4189043 & 93.73 & 261371 & 90.16 \\
\hline
\end{tabular}




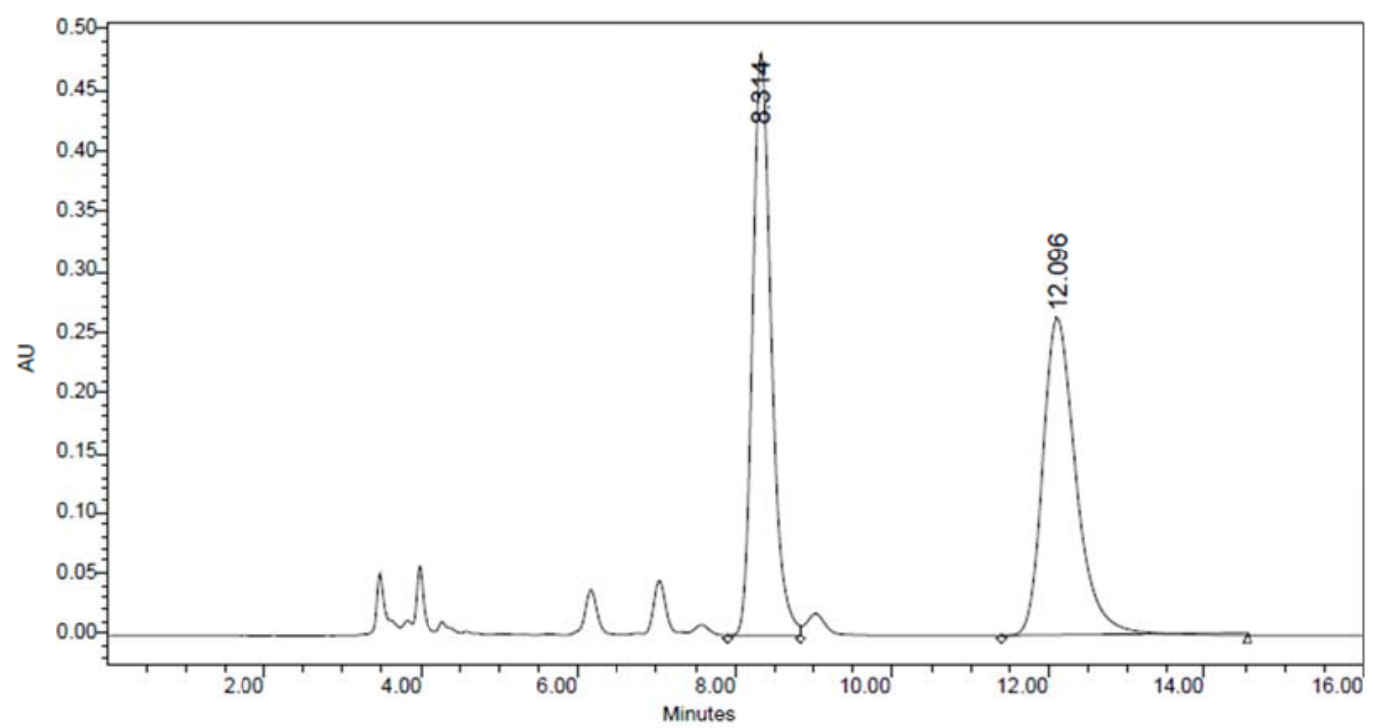

\begin{tabular}{|c|c|c|c|c|c|}
\hline & $\begin{array}{c}\text { RT } \\
(\mathrm{min})\end{array}$ & $\begin{array}{c}\text { Area } \\
\left(\mathrm{V}^{*} \mathrm{sec}\right)\end{array}$ & \% Area & $\begin{array}{c}\text { Height } \\
(\mathrm{V})\end{array}$ & $\begin{array}{c}\% \\
\text { Height }\end{array}$ \\
\hline 1 & 8.314 & 7932825 & 50.61 & 480886 & 64.67 \\
\hline 2 & 12.096 & 7740847 & 49.39 & 262746 & 35.33 \\
\hline
\end{tabular}
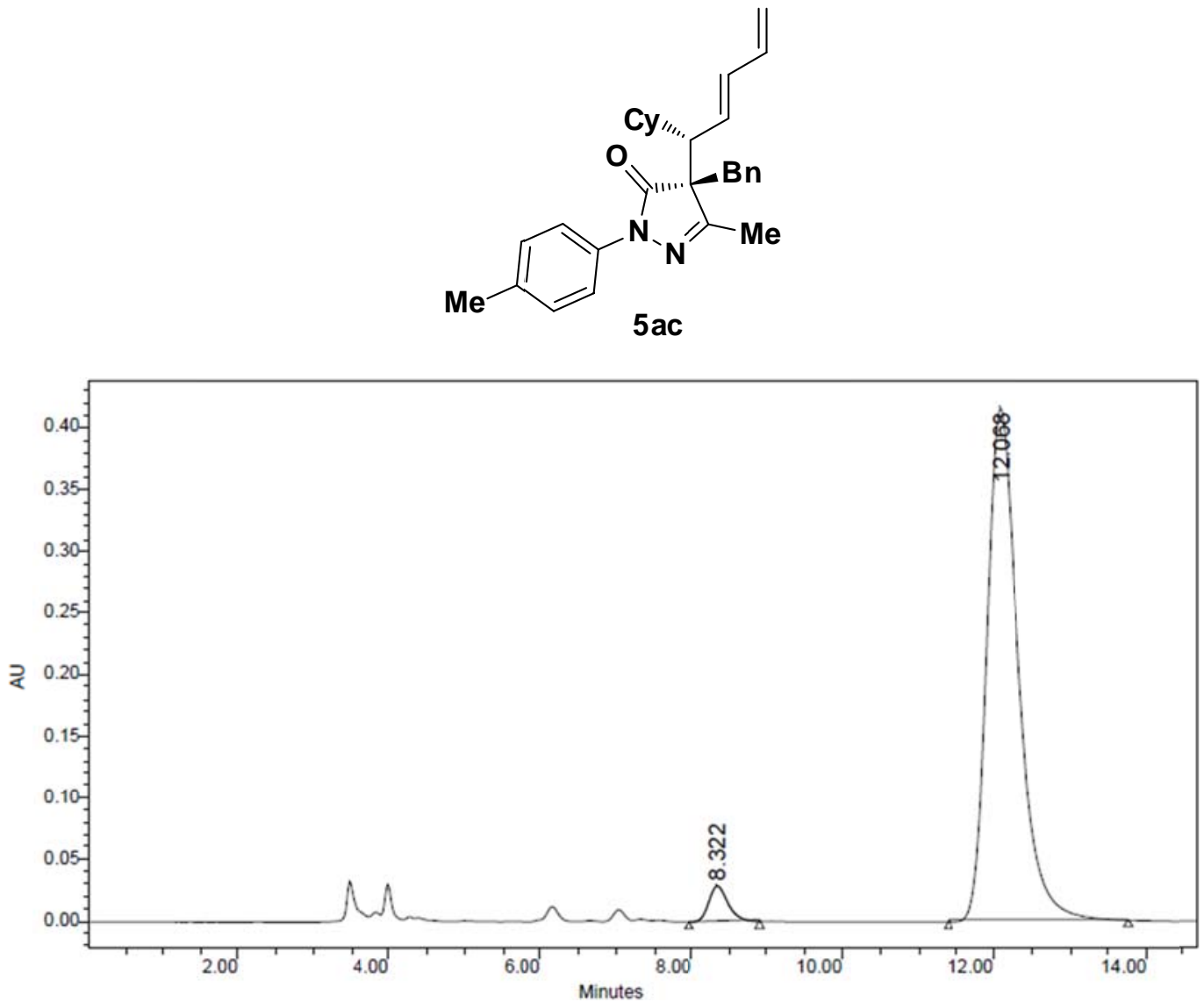

\begin{tabular}{|c|c|c|r|r|r|}
\hline & $\begin{array}{c}\text { RT } \\
(\mathrm{min})\end{array}$ & $\begin{array}{c}\text { Area } \\
\left(\mathrm{V}^{*} \mathrm{sec}\right)\end{array}$ & $\%$ Area & $\begin{array}{c}\text { Height } \\
(\mathrm{V})\end{array}$ & $\begin{array}{c}\% \\
\text { Height }\end{array}$ \\
\hline 1 & 8.322 & 508659 & 4.03 & 28868 & 6.48 \\
\hline 2 & 12.068 & 12120511 & 95.97 & 416437 & 93.52 \\
\hline
\end{tabular}




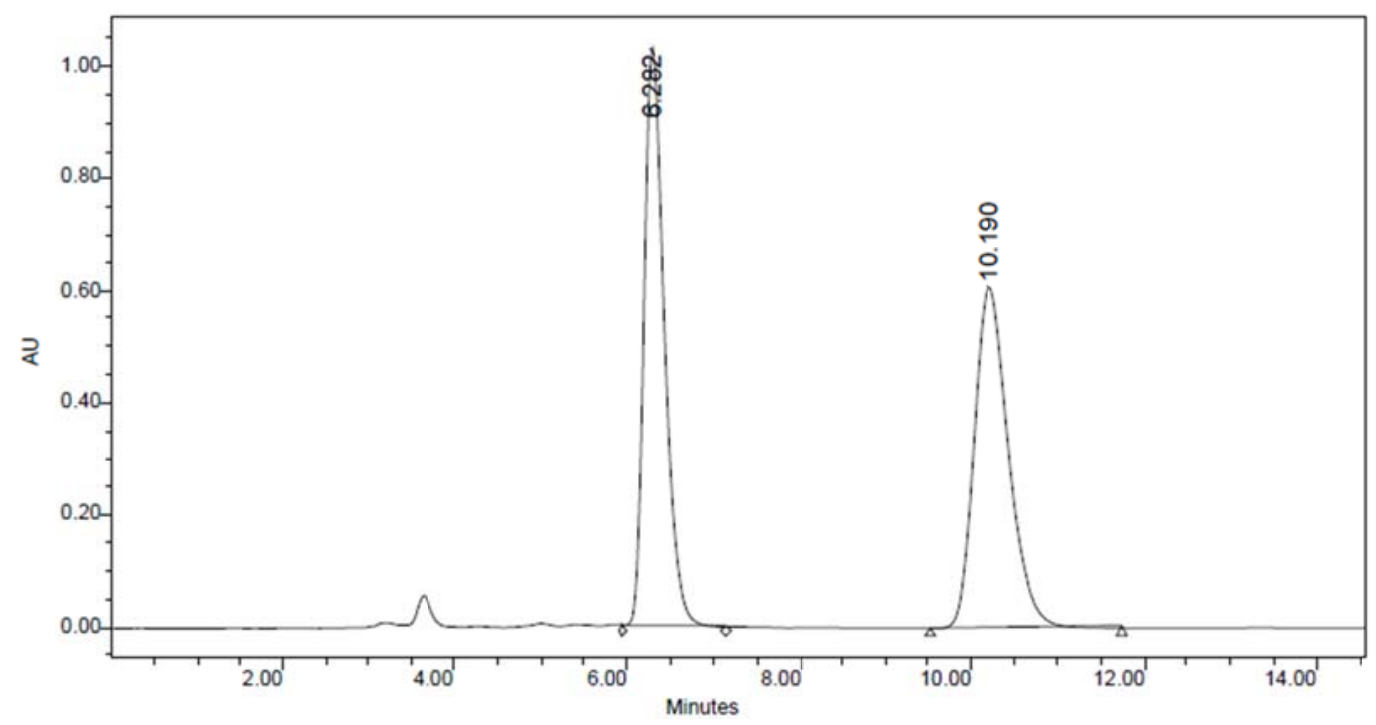

\begin{tabular}{|c|c|c|c|c|c|}
\hline & $\begin{array}{c}\mathrm{RT} \\
(\mathrm{min})\end{array}$ & $\begin{array}{c}\text { Area } \\
\left(\mathrm{V}^{*} \mathrm{sec}\right)\end{array}$ & $\%$ Area & $\begin{array}{c}\text { Height } \\
(\mathrm{V})\end{array}$ & $\begin{array}{c}\% \\
\text { Height }\end{array}$ \\
\hline 1 & 6.282 & 16936101 & 49.86 & 1035003 & 62.98 \\
\hline 2 & 10.190 & 17029667 & 50.14 & 608397 & 37.02 \\
\hline
\end{tabular}
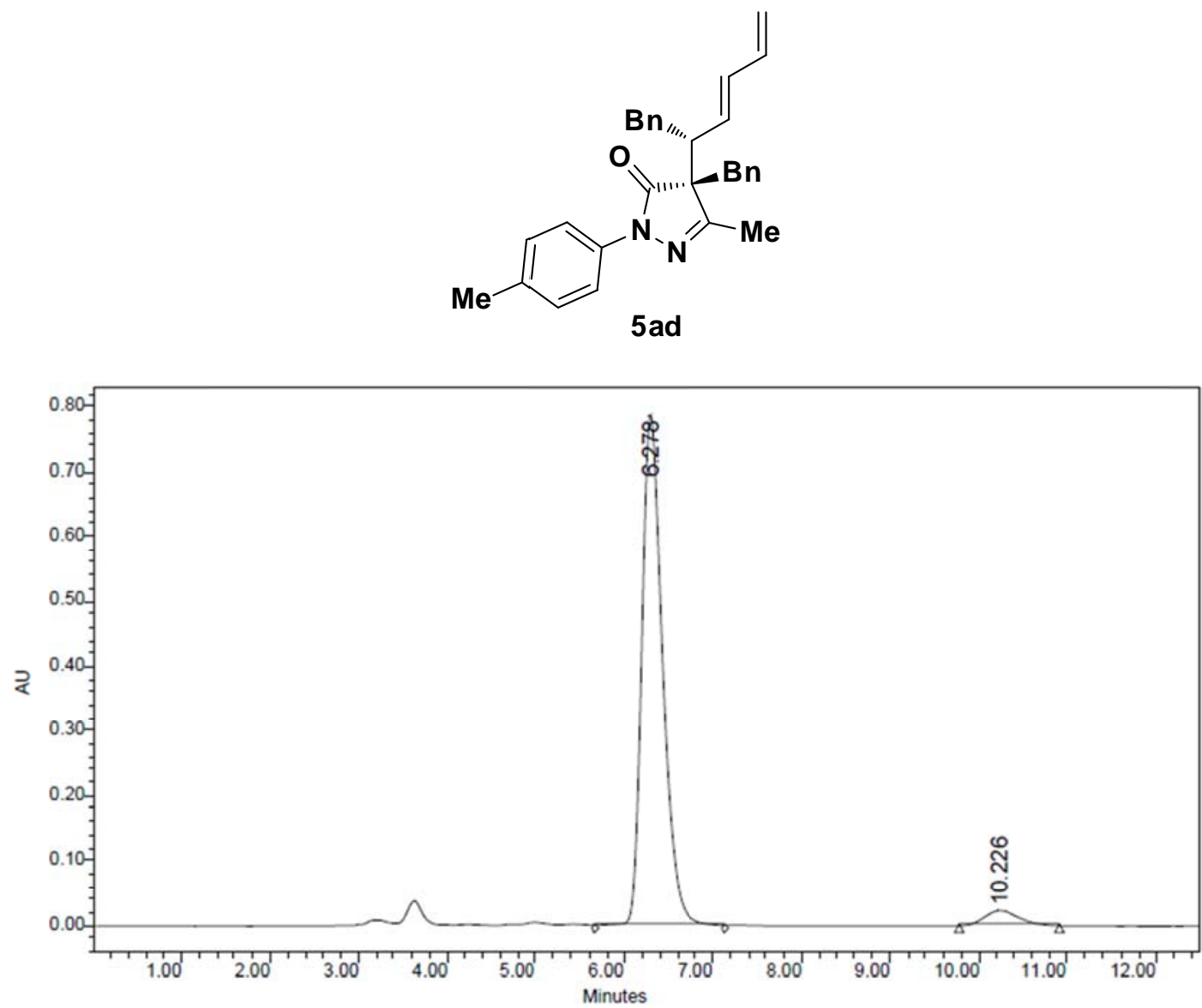

\begin{tabular}{|c|c|c|r|r|r|}
\hline & $\begin{array}{c}\text { RT } \\
(\mathrm{min})\end{array}$ & $\begin{array}{c}\text { Area } \\
\left(\mathrm{V}^{*} \mathrm{sec}\right)\end{array}$ & \% Area & $\begin{array}{c}\text { Height } \\
(\mathrm{V})\end{array}$ & $\begin{array}{c}\% \\
\text { Height }\end{array}$ \\
\hline 1 & 6.278 & 12896043 & 95.25 & 789591 & 97.11 \\
\hline 2 & 10.226 & 642536 & 4.75 & 23469 & 2.89 \\
\hline
\end{tabular}




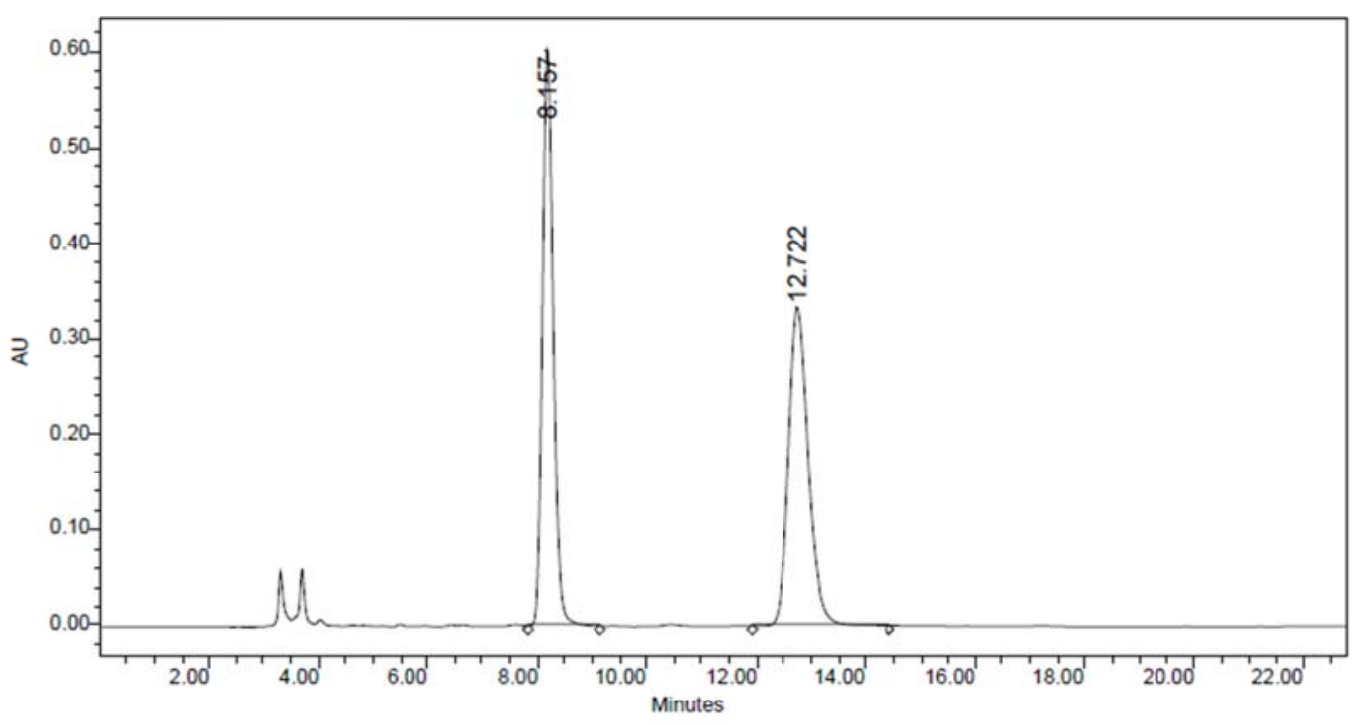

\begin{tabular}{|c|c|c|c|c|c|}
\hline & $\begin{array}{c}\text { RT } \\
(\mathrm{min})\end{array}$ & $\begin{array}{c}\text { Area } \\
\left(\mathrm{V}^{*} \mathrm{sec}\right)\end{array}$ & $\%$ Area & $\begin{array}{c}\text { Height } \\
(\mathrm{V})\end{array}$ & $\begin{array}{c}\% \\
\text { Height }\end{array}$ \\
\hline 1 & 8.157 & 8384105 & 49.63 & 604563 & 64.47 \\
\hline 2 & 12.722 & 8509676 & 50.37 & 333247 & 35.53 \\
\hline
\end{tabular}

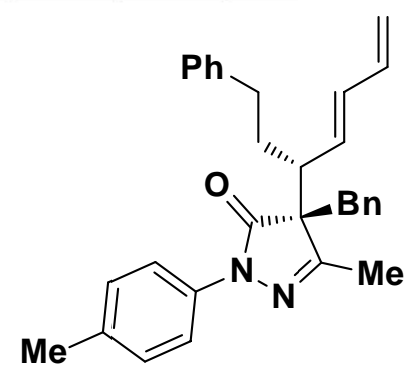

$5 a e$

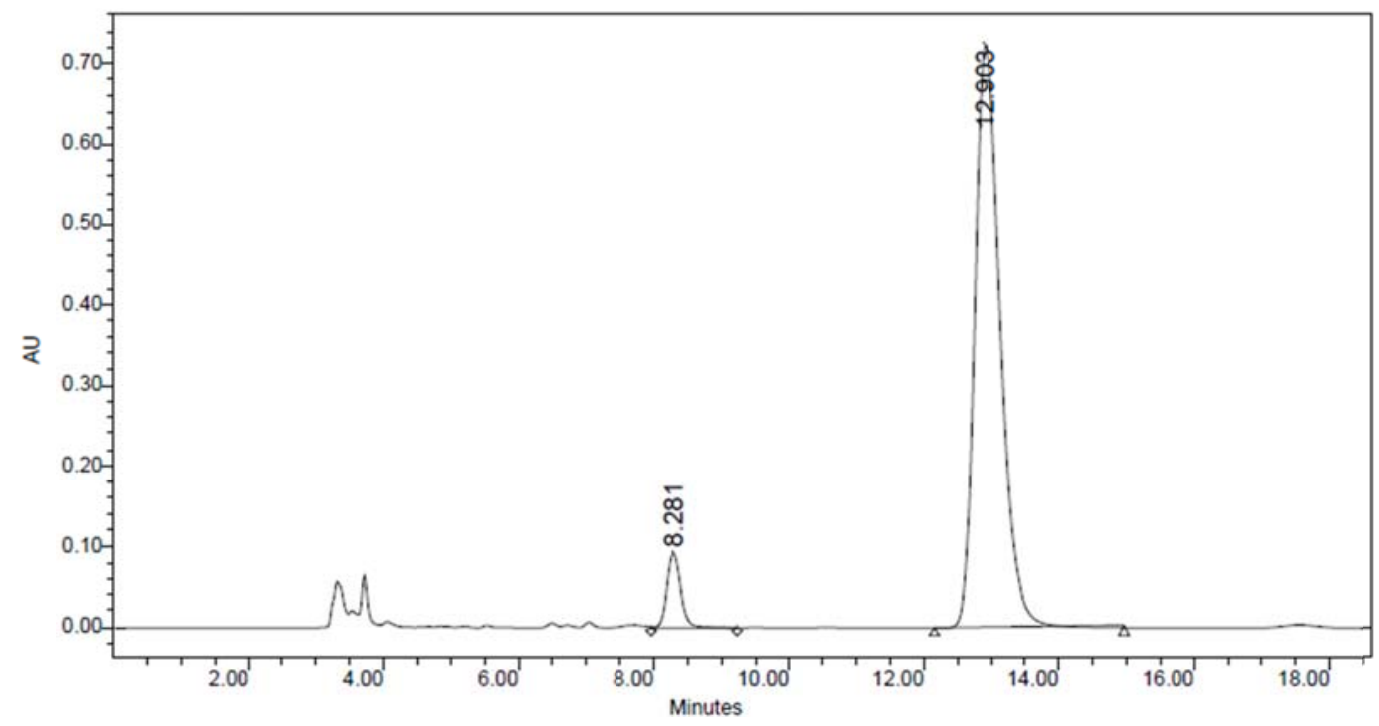

\begin{tabular}{|c|c|c|r|r|r|}
\hline & $\begin{array}{c}\text { RT } \\
(\mathrm{min})\end{array}$ & $\begin{array}{c}\text { Area } \\
\left(\mathrm{V}^{*} \mathrm{sec}\right)\end{array}$ & $\%$ Area & $\begin{array}{c}\text { Height } \\
(\mathrm{V})\end{array}$ & $\begin{array}{c}\% \\
\text { Height }\end{array}$ \\
\hline 1 & 8.281 & 1326920 & 6.53 & 92790 & 11.35 \\
\hline 2 & 12.903 & 18983960 & 93.47 & 724848 & 88.65 \\
\hline
\end{tabular}




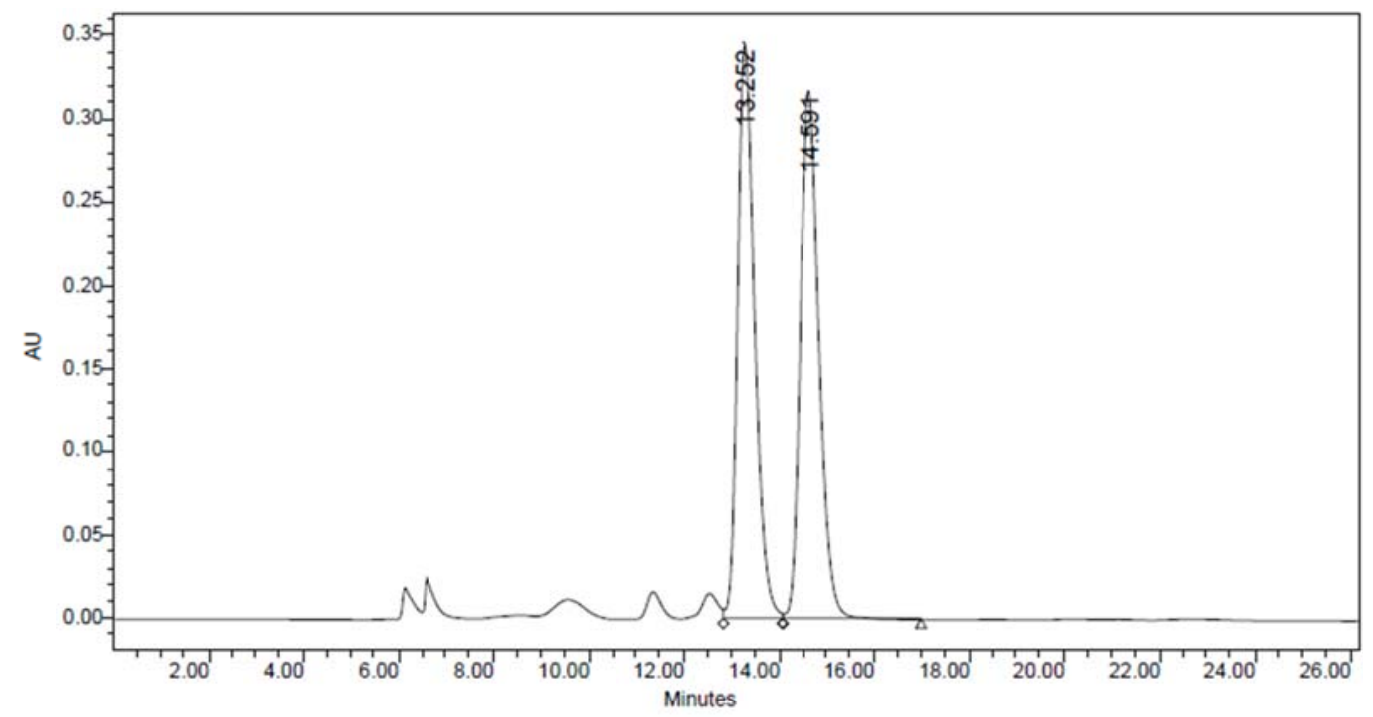

\begin{tabular}{|c|c|c|c|c|c|}
\hline & $\begin{array}{c}\text { RT } \\
(\mathrm{min})\end{array}$ & $\begin{array}{c}\text { Area } \\
\left(\mathrm{V}^{*} \mathrm{sec}\right)\end{array}$ & \% Area & $\begin{array}{c}\text { Height } \\
(\mathrm{V})\end{array}$ & $\begin{array}{c}\% \\
\text { Height }\end{array}$ \\
\hline 1 & 13.252 & 8607676 & 49.73 & 345841 & 52.09 \\
\hline 2 & 14.591 & 8702081 & 50.27 & 318094 & 47.91 \\
\hline
\end{tabular}
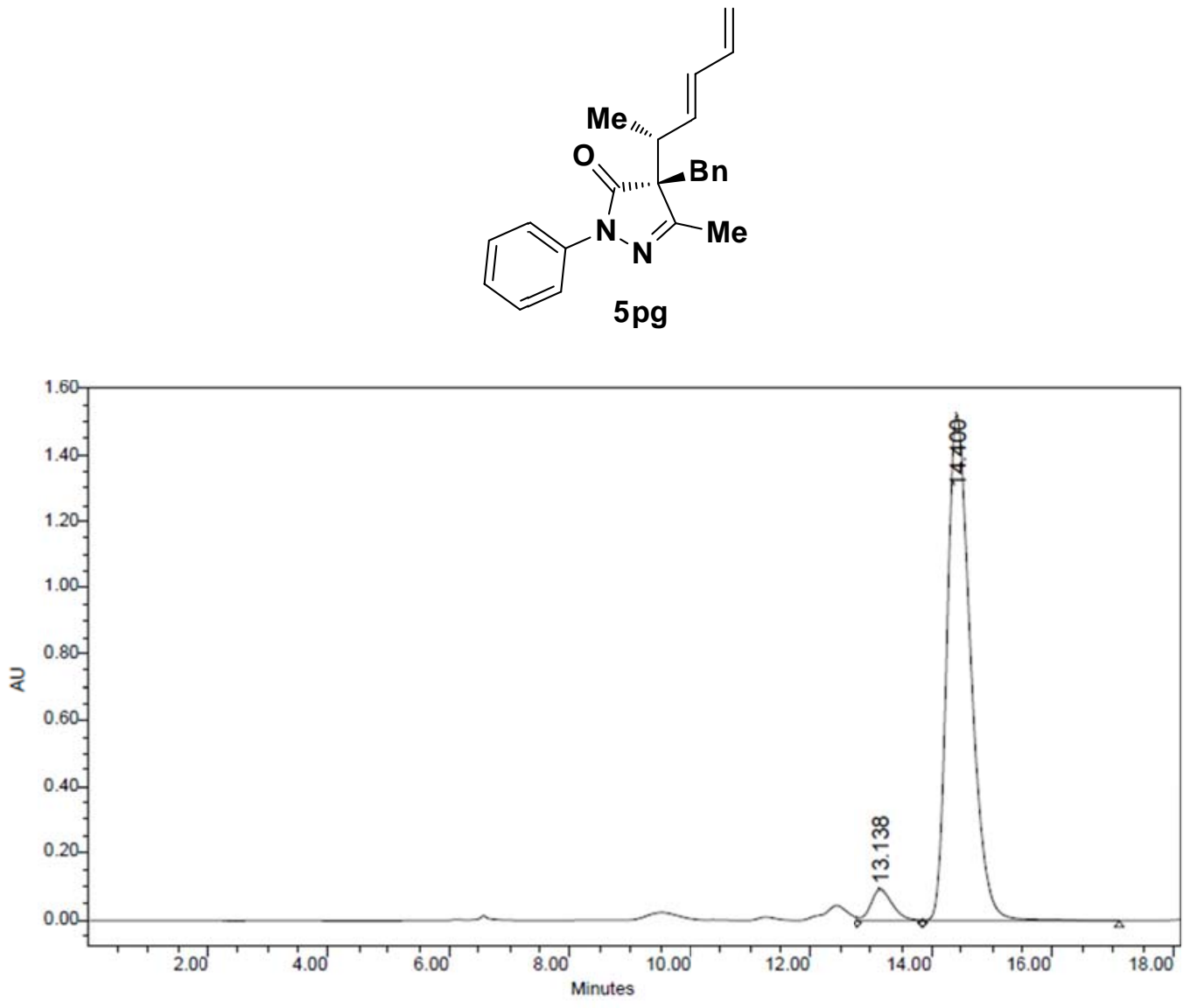

\begin{tabular}{|c|c|c|r|r|r|}
\hline & $\begin{array}{c}\text { RT } \\
(\mathrm{min})\end{array}$ & $\begin{array}{c}\text { Area } \\
\left(\mathrm{V}^{*} \mathrm{sec}\right)\end{array}$ & \% Area & $\begin{array}{c}\text { Height } \\
(\mathrm{V})\end{array}$ & $\begin{array}{c}\% \\
\text { Height }\end{array}$ \\
\hline 1 & 13.138 & 2301417 & 5.31 & 93473 & 5.78 \\
\hline 2 & 14.400 & 40999603 & 94.69 & 1524231 & 94.22 \\
\hline
\end{tabular}




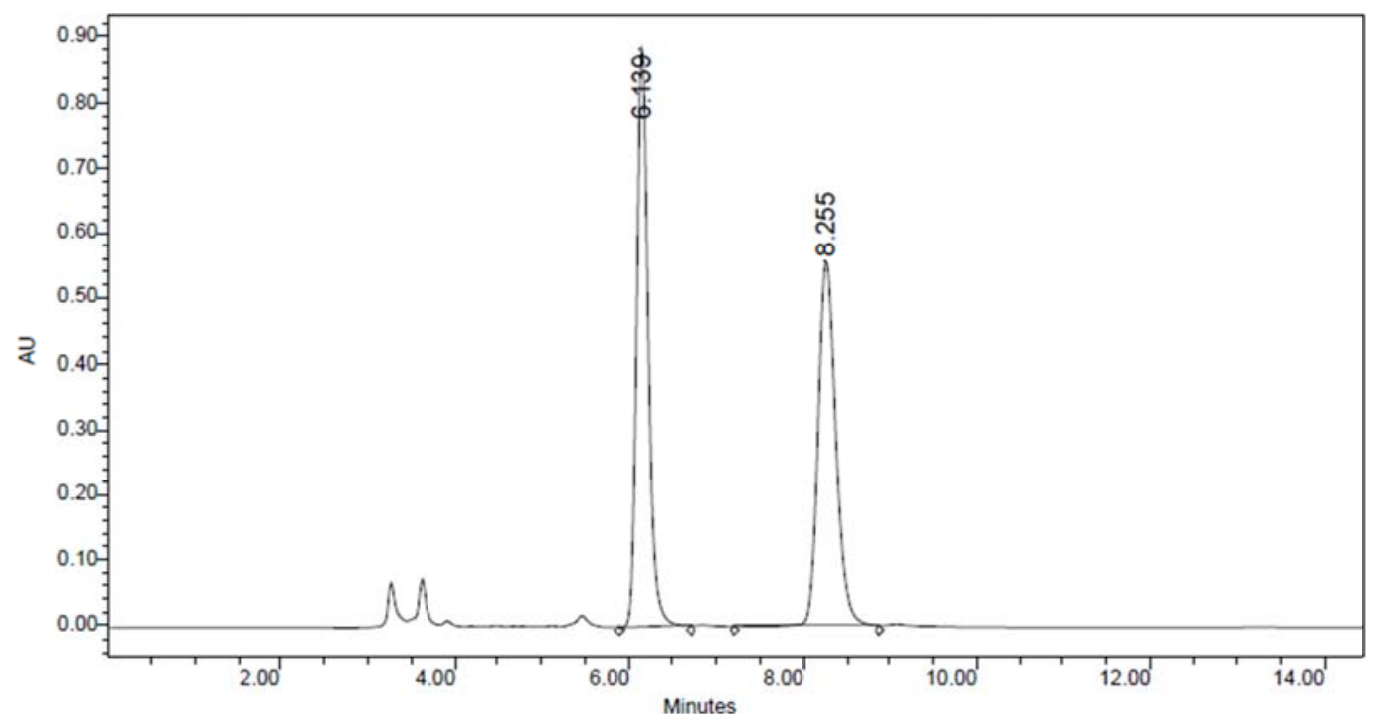

\begin{tabular}{|c|c|c|c|c|c|}
\hline & $\begin{array}{c}\text { RT } \\
(\mathrm{min})\end{array}$ & $\begin{array}{c}\text { Area } \\
\left(\mathrm{V}^{*} \mathrm{sec}\right)\end{array}$ & $\%$ Area & $\begin{array}{c}\text { Height } \\
(\mathrm{V})\end{array}$ & $\begin{array}{c}\% \\
\text { Height }\end{array}$ \\
\hline 1 & 6.139 & 8229268 & 50.23 & 890727 & 61.42 \\
\hline 2 & 8.255 & 8154142 & 49.77 & 559454 & 38.58 \\
\hline
\end{tabular}
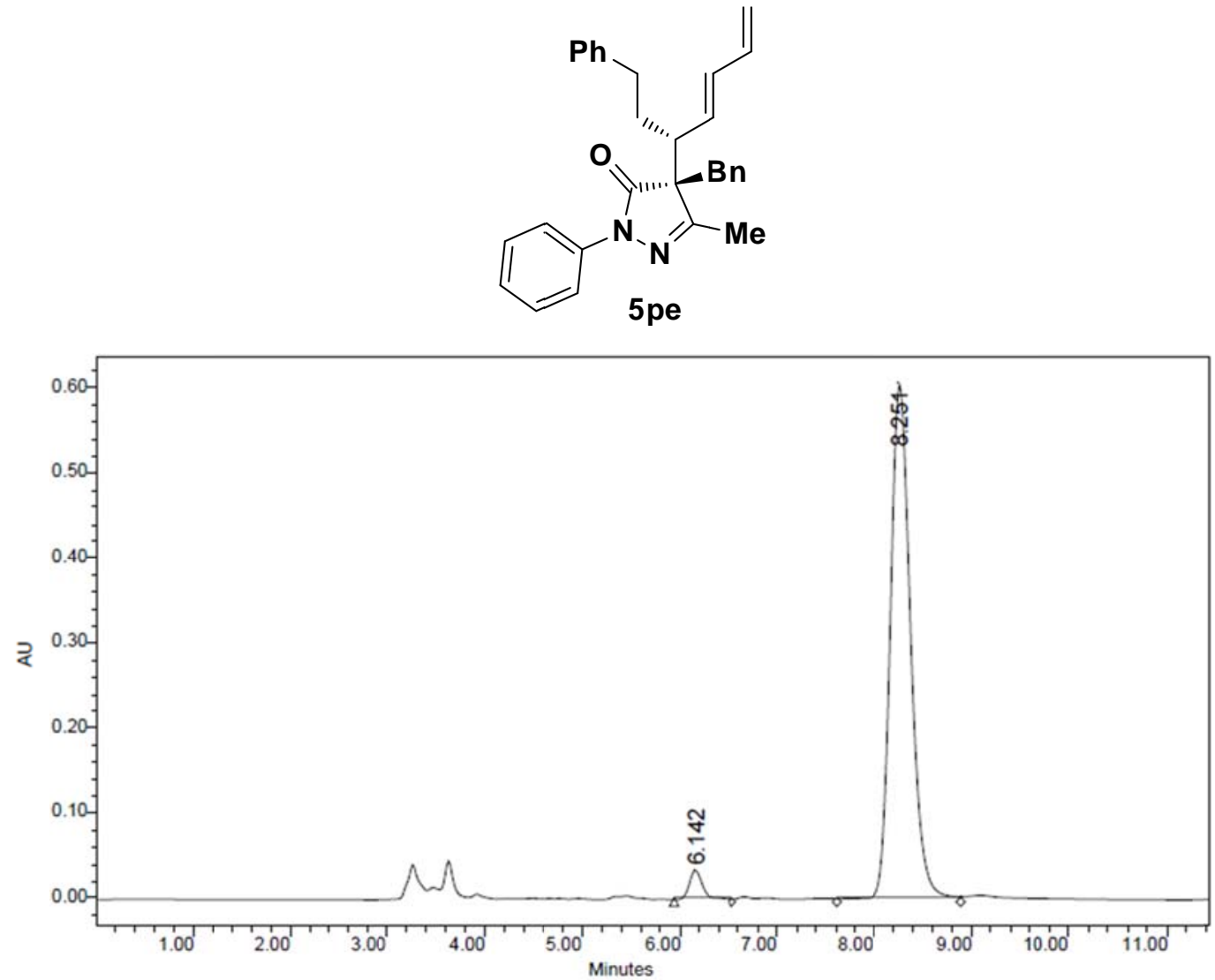

\begin{tabular}{|l|c|r|r|r|r|}
\hline & $\begin{array}{c}\text { RT } \\
(\mathrm{min})\end{array}$ & $\begin{array}{c}\text { Area } \\
\left(\mathrm{V}^{*} \mathrm{sec}\right)\end{array}$ & $\%$ Area & $\begin{array}{c}\text { Height } \\
(\mathrm{V})\end{array}$ & $\begin{array}{c}\% \\
\text { Height }\end{array}$ \\
\hline 1 & 6.142 & 325158 & 3.63 & 34339 & 5.37 \\
\hline 2 & 8.251 & 8640411 & 96.37 & 604713 & 94.63 \\
\hline
\end{tabular}




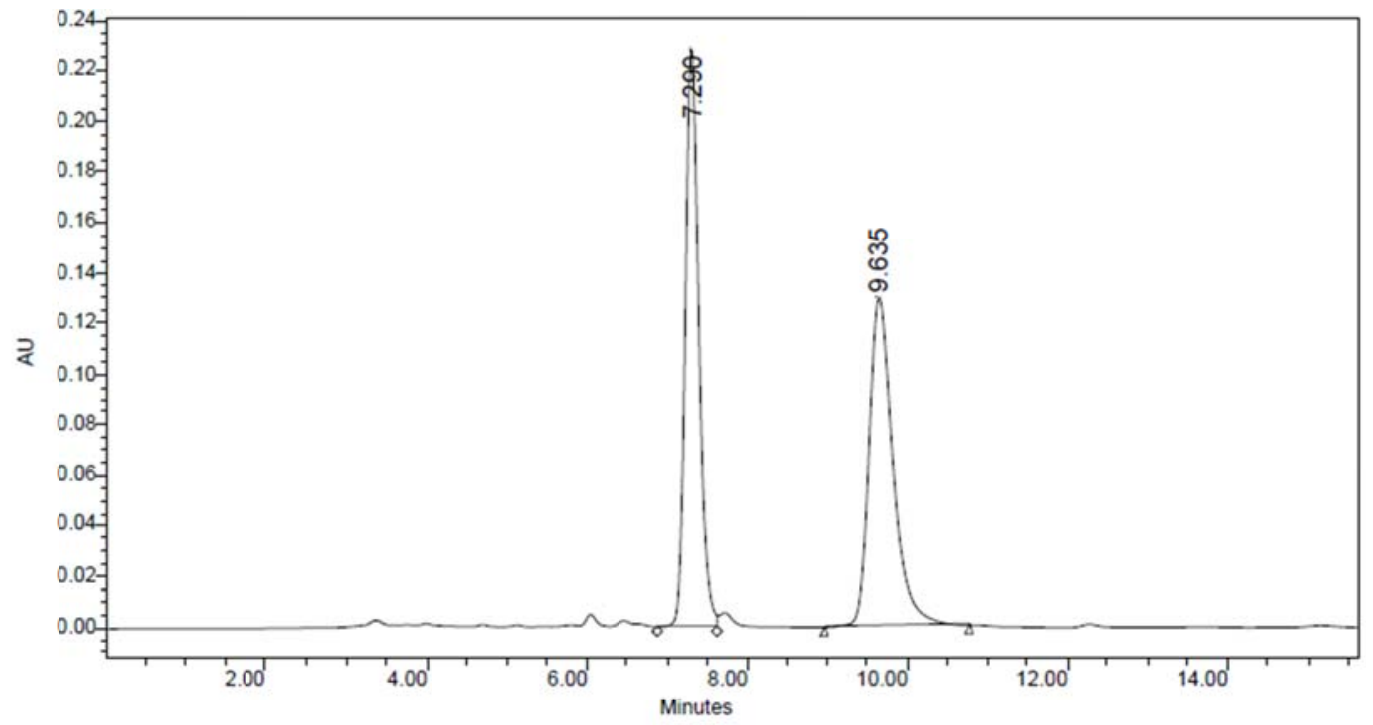

\begin{tabular}{|c|c|c|c|c|c|}
\hline & $\begin{array}{c}\text { RT } \\
(\mathrm{min})\end{array}$ & $\begin{array}{c}\text { Area } \\
\left(\mathrm{V}^{*} \mathrm{sec}\right)\end{array}$ & \% Area & $\begin{array}{c}\text { Height } \\
(\mathrm{V})\end{array}$ & $\begin{array}{c}\% \\
\text { Height }\end{array}$ \\
\hline 1 & 7.290 & 2685310 & 49.78 & 229463 & 63.88 \\
\hline 2 & 9.635 & 2708529 & 50.22 & 129744 & 36.12 \\
\hline
\end{tabular}
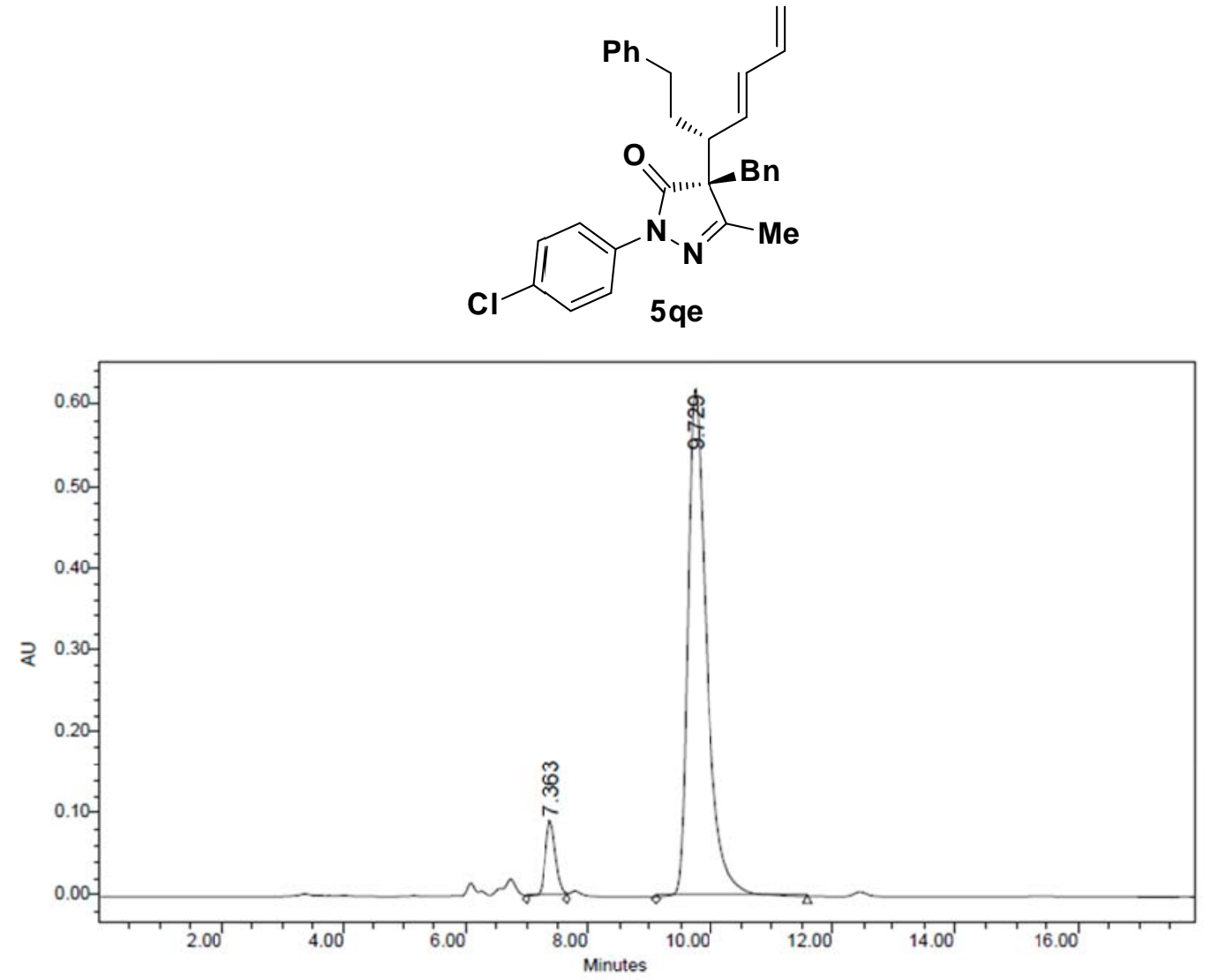

\begin{tabular}{|c|c|c|r|r|r|}
\hline & $\begin{array}{c}\text { RT } \\
(\mathrm{min})\end{array}$ & $\begin{array}{c}\text { Area } \\
\left(\mathrm{V}^{*} \mathrm{sec}\right)\end{array}$ & $\%$ Area & $\begin{array}{l}\text { Height } \\
(\mathrm{V})\end{array}$ & $\begin{array}{c}\% \\
\text { Height }\end{array}$ \\
\hline 1 & 7.363 & 1104130 & 7.67 & 90795 & 12.77 \\
\hline 2 & 9.729 & 13284632 & 92.33 & 619964 & 87.23 \\
\hline
\end{tabular}




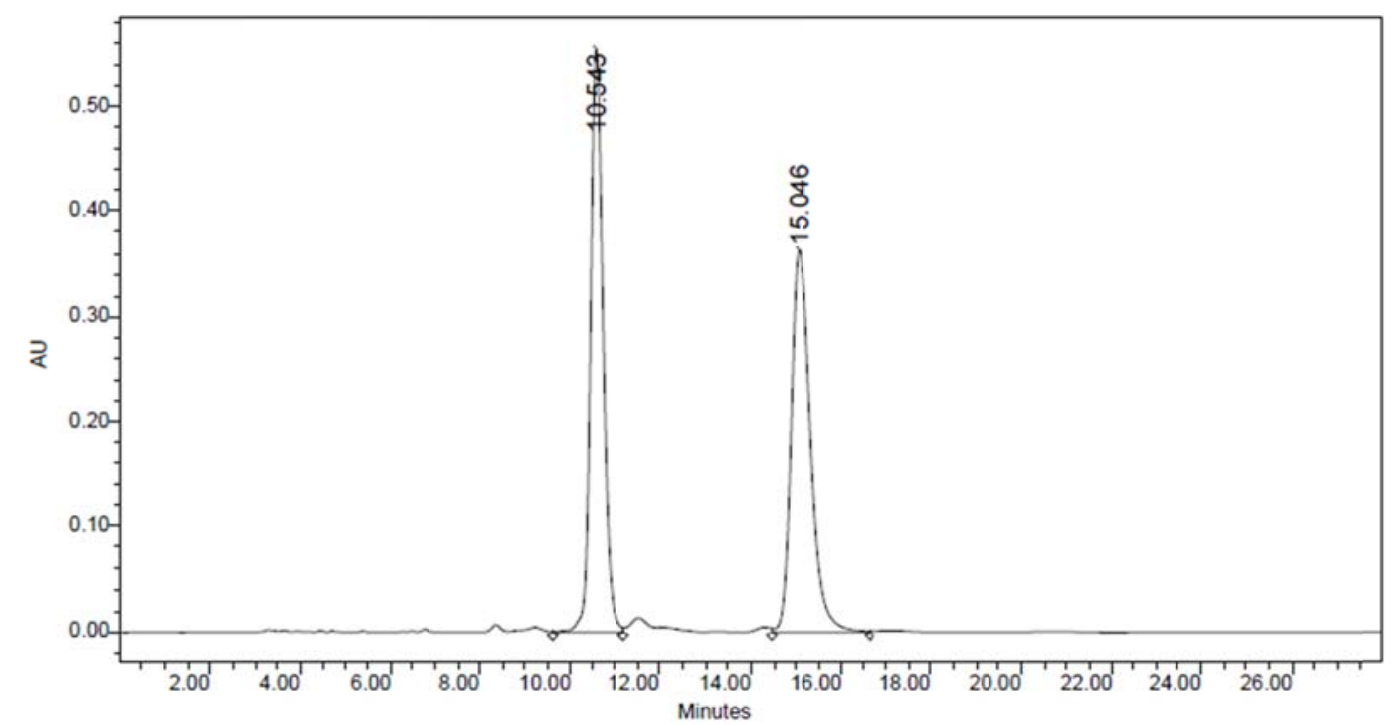

\begin{tabular}{|c|c|c|c|c|c|}
\hline & $\begin{array}{c}\mathrm{RT} \\
(\mathrm{min})\end{array}$ & $\begin{array}{c}\text { Area } \\
\left(\mathrm{V}^{*} \mathrm{sec}\right)\end{array}$ & $\%$ Area & $\begin{array}{c}\text { Height } \\
(\mathrm{V})\end{array}$ & $\begin{array}{c}\% \\
\text { Height }\end{array}$ \\
\hline 1 & 10.543 & 10790722 & 49.82 & 557470 & 60.40 \\
\hline 2 & 15.046 & 10869025 & 50.18 & 365463 & 39.60 \\
\hline
\end{tabular}
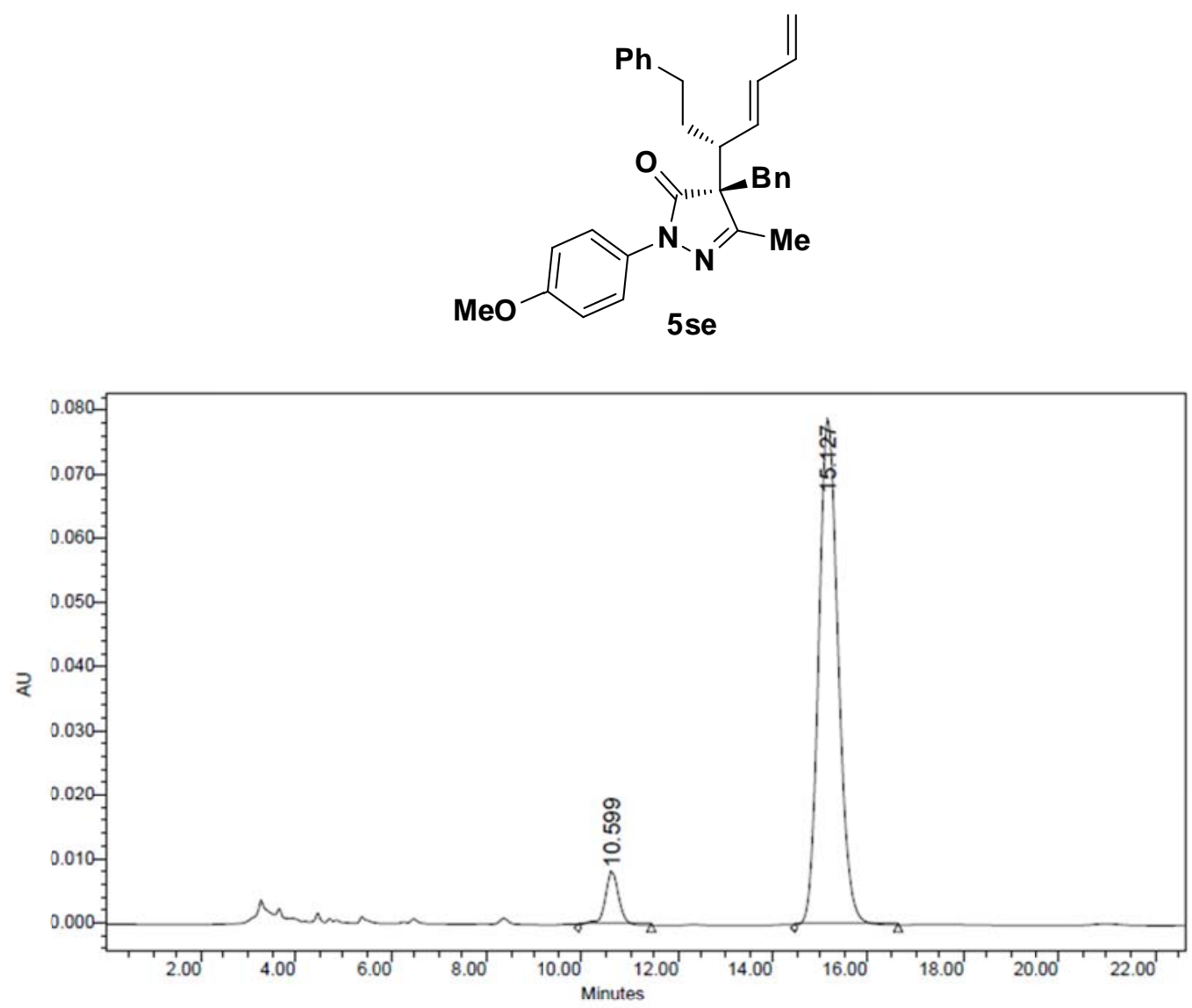

\begin{tabular}{|l|c|r|r|r|r|}
\hline & $\begin{array}{c}\text { RT } \\
(\mathrm{min})\end{array}$ & $\begin{array}{c}\text { Area } \\
\left(\mathrm{V}^{*} \mathrm{sec}\right)\end{array}$ & \% Area & $\begin{array}{l}\text { Height } \\
(\mathrm{V})\end{array}$ & $\begin{array}{c}\% \\
\text { Height }\end{array}$ \\
\hline 1 & 10.599 & 166975 & 6.93 & 8288 & 9.52 \\
\hline 2 & 15.127 & 2241823 & 93.07 & 78809 & 90.48 \\
\hline
\end{tabular}




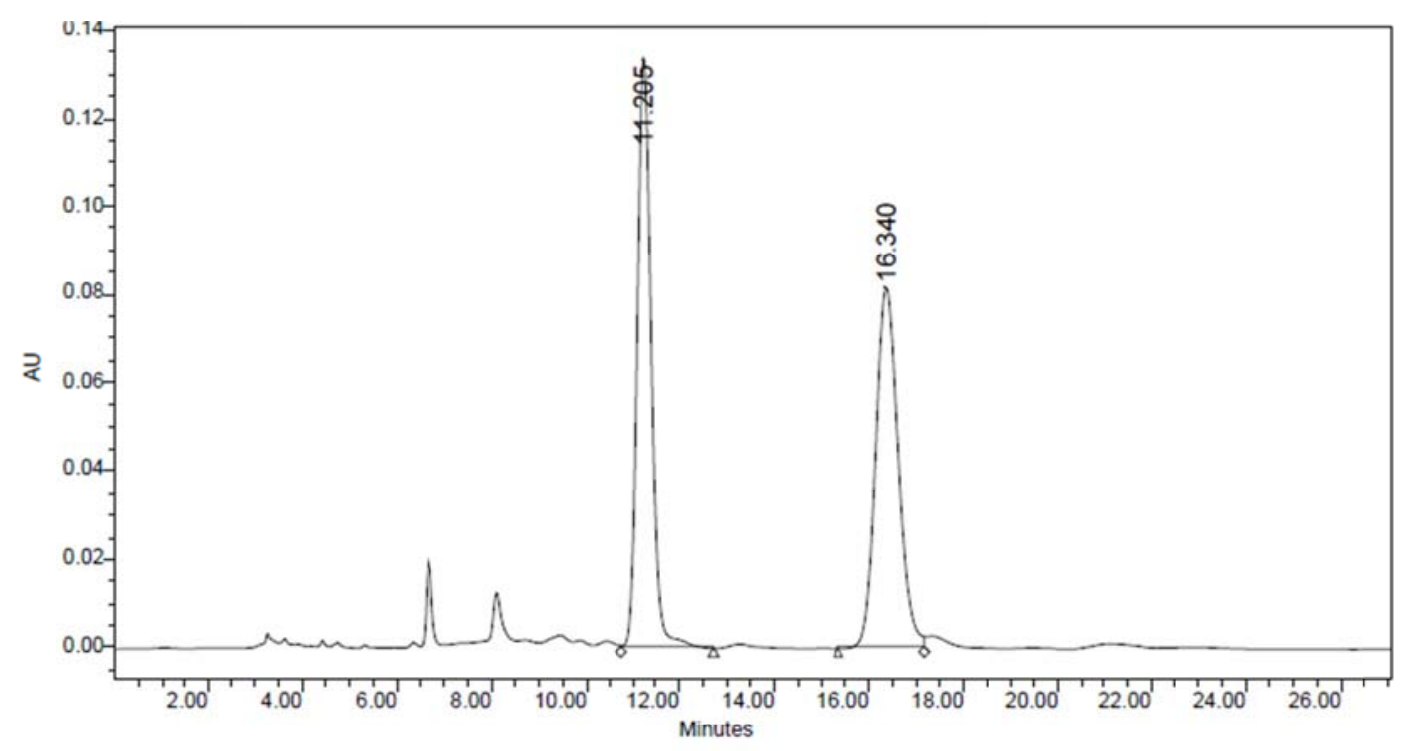

\begin{tabular}{|c|c|c|c|c|c|}
\hline & $\begin{array}{c}\text { RT } \\
(\mathrm{min})\end{array}$ & $\begin{array}{c}\text { Area } \\
\left(\mathrm{V}^{*} \mathrm{sec}\right)\end{array}$ & $\%$ Area & $\begin{array}{c}\text { Height } \\
(\mathrm{V})\end{array}$ & $\begin{array}{c}\% \\
\text { Height }\end{array}$ \\
\hline 1 & 11.205 & 2750975 & 50.23 & 133901 & 62.09 \\
\hline 2 & 16.340 & 2725446 & 49.77 & 81768 & 37.91 \\
\hline
\end{tabular}<smiles>C=C/C=C/C(CCc1ccccc1)[C@]1(Cc2ccc(OC)cc2)C(=O)N(c2ccc(C)cc2)N=C1C</smiles>

5 de

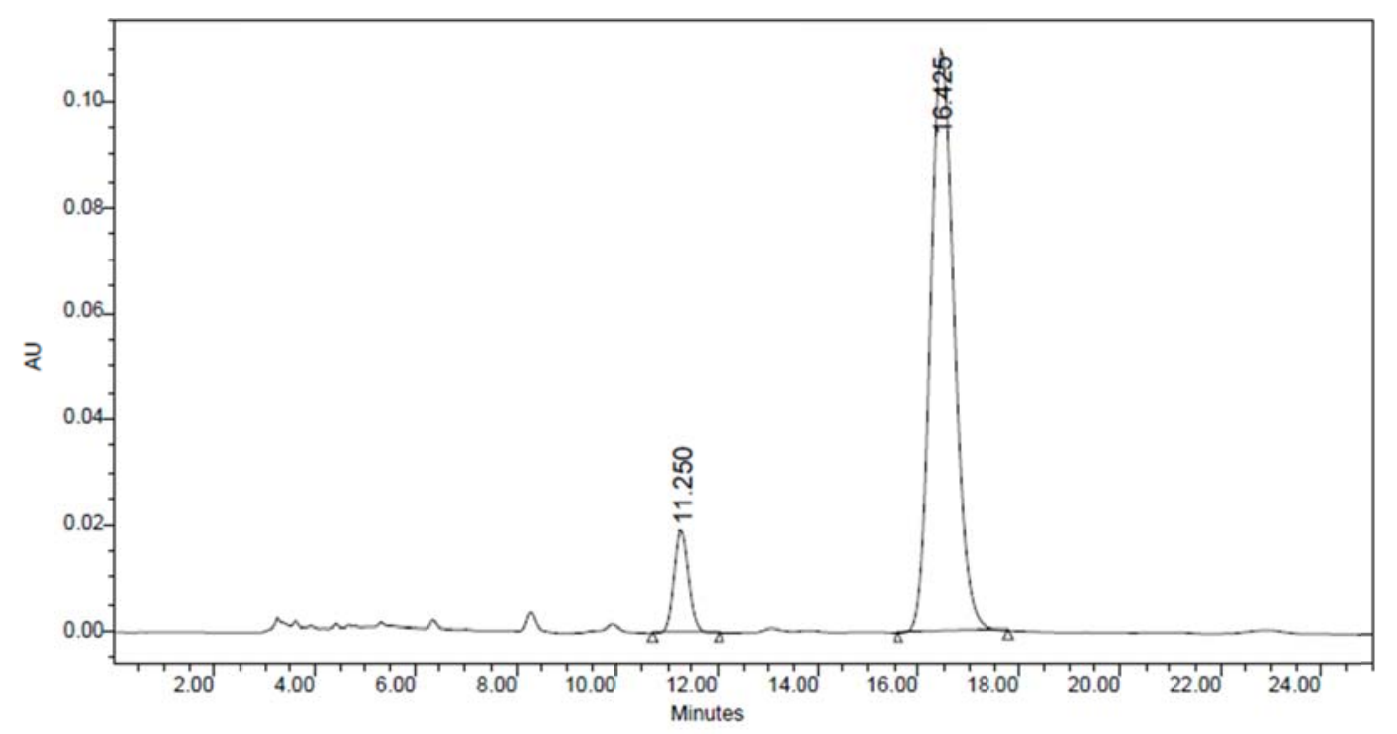

\begin{tabular}{|c|c|c|r|r|c|}
\hline & $\begin{array}{c}\text { RT } \\
(\mathrm{min})\end{array}$ & $\begin{array}{c}\text { Area } \\
\left(\mathrm{V}^{*} \mathrm{sec}\right)\end{array}$ & $\%$ Area & $\begin{array}{c}\text { Height } \\
(\mathrm{V})\end{array}$ & $\begin{array}{c}\% \\
\text { Height }\end{array}$ \\
\hline 1 & 11.250 & 386255 & 9.57 & 19305 & 14.96 \\
\hline 2 & 16.425 & 3650357 & 90.43 & 109761 & 85.04 \\
\hline
\end{tabular}




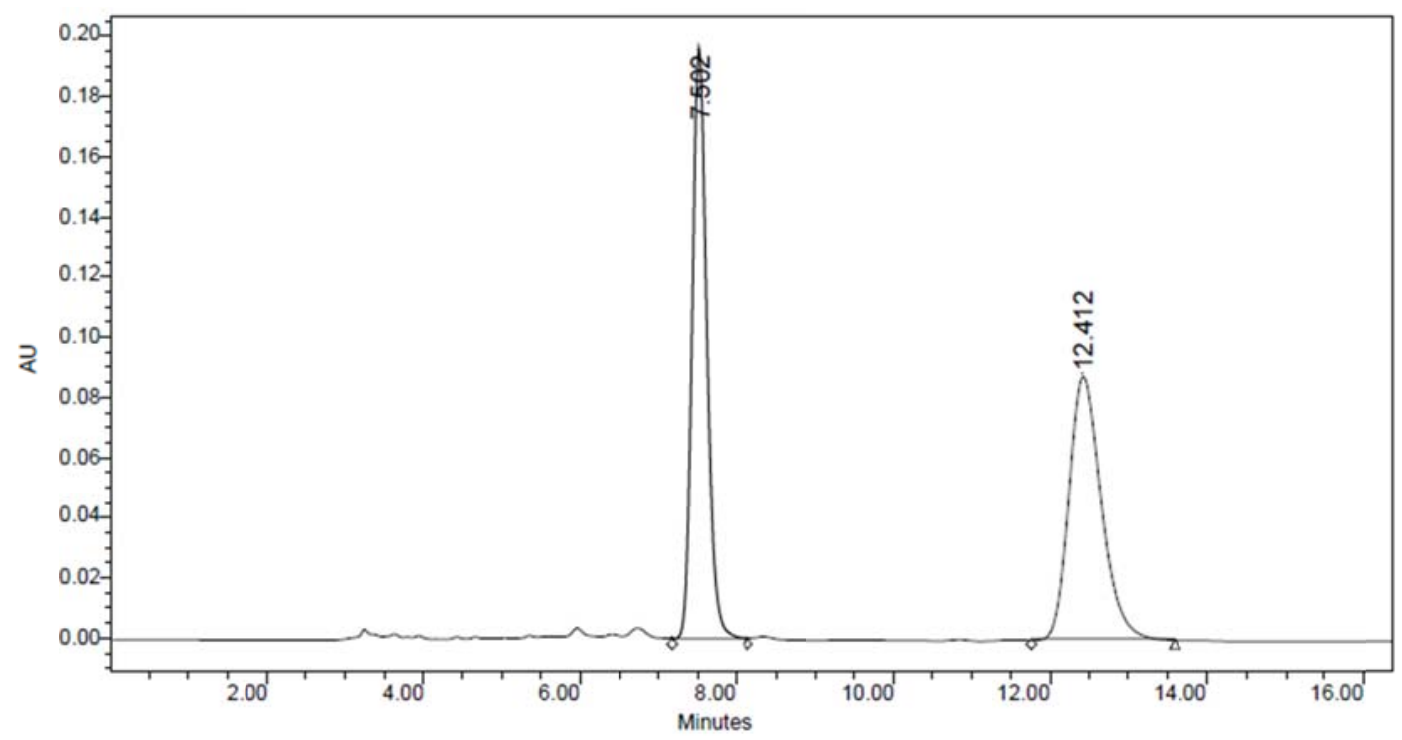

\begin{tabular}{|c|c|c|r|r|c|}
\hline & $\begin{array}{c}\text { RT } \\
(\mathrm{min})\end{array}$ & $\begin{array}{c}\text { Area } \\
\left(\mathrm{V}^{*} \mathrm{sec}\right)\end{array}$ & \% Area & $\begin{array}{c}\text { Height } \\
(\mathrm{V})\end{array}$ & $\begin{array}{c}\% \\
\text { Height }\end{array}$ \\
\hline 1 & 7.502 & 2568356 & 50.45 & 196580 & 69.13 \\
\hline 2 & 12.412 & 2522887 & 49.55 & 87768 & 30.87 \\
\hline
\end{tabular}
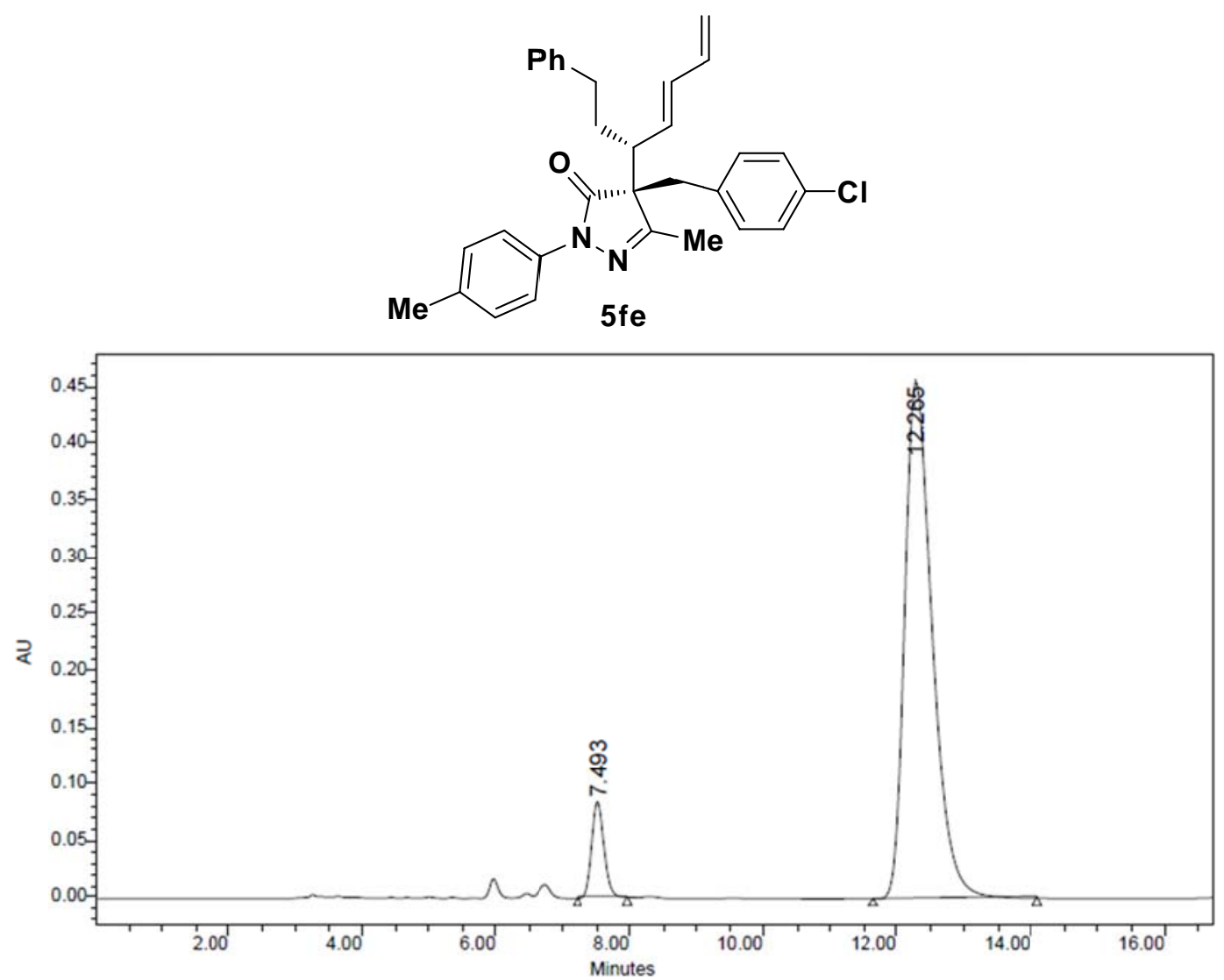

\begin{tabular}{|c|c|c|r|r|r|}
\hline & $\begin{array}{c}\text { RT } \\
(\mathrm{min})\end{array}$ & $\begin{array}{c}\text { Area } \\
\left(\mathrm{V}^{*} \mathrm{sec}\right)\end{array}$ & \% Area & $\begin{array}{c}\text { Height } \\
(\mathrm{V})\end{array}$ & $\begin{array}{c}\% \\
\text { Height }\end{array}$ \\
\hline 1 & 7.493 & 1077900 & 7.70 & 84496 & 15.66 \\
\hline 2 & 12.265 & 12928580 & 92.30 & 455099 & 84.34 \\
\hline
\end{tabular}




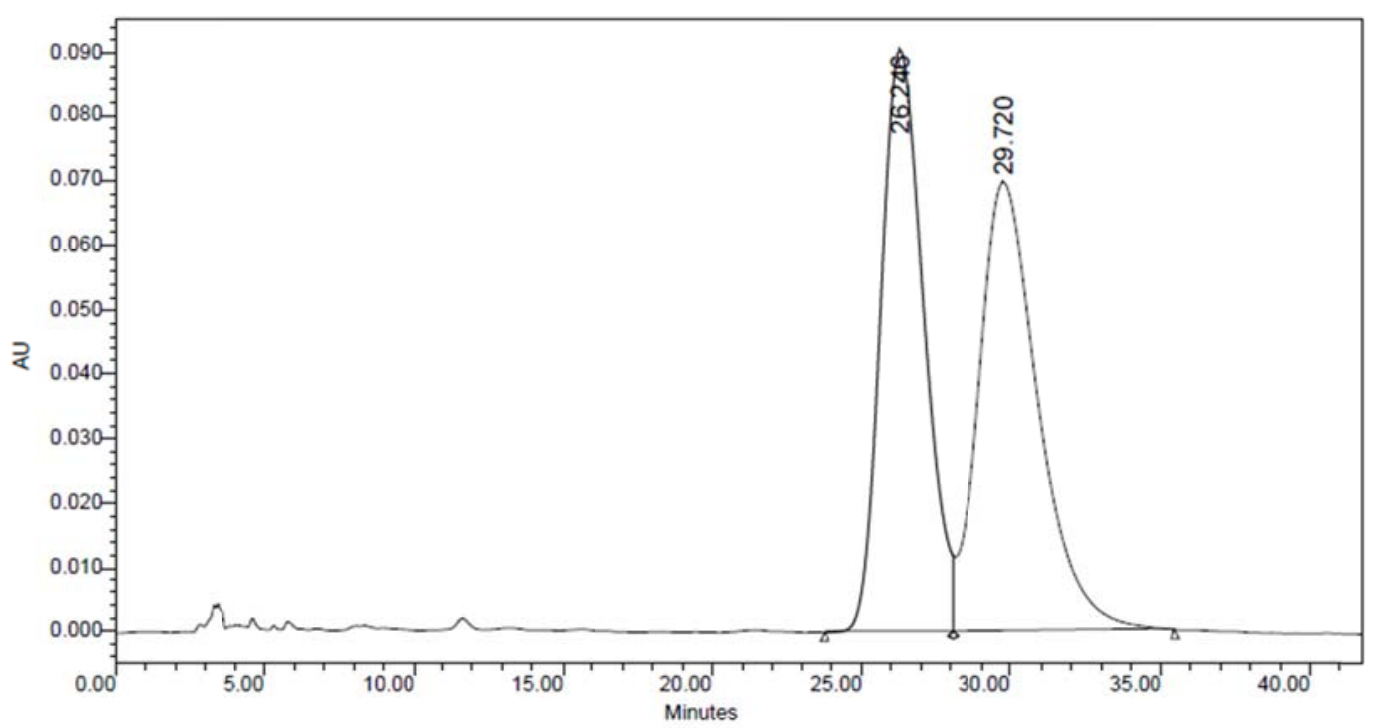

\begin{tabular}{|c|c|c|c|c|c|}
\hline & $\begin{array}{c}\text { RT } \\
(\mathrm{min})\end{array}$ & $\begin{array}{c}\text { Area } \\
\left(\mathrm{V}^{*} \mathrm{sec}\right)\end{array}$ & $\%$ Area & $\begin{array}{c}\text { Height } \\
(\mathrm{V})\end{array}$ & $\begin{array}{c}\% \\
\text { Height }\end{array}$ \\
\hline 1 & 26.246 & 9098132 & 48.65 & 90359 & 56.46 \\
\hline 2 & 29.720 & 9602512 & 51.35 & 69687 & 43.54 \\
\hline
\end{tabular}

Me
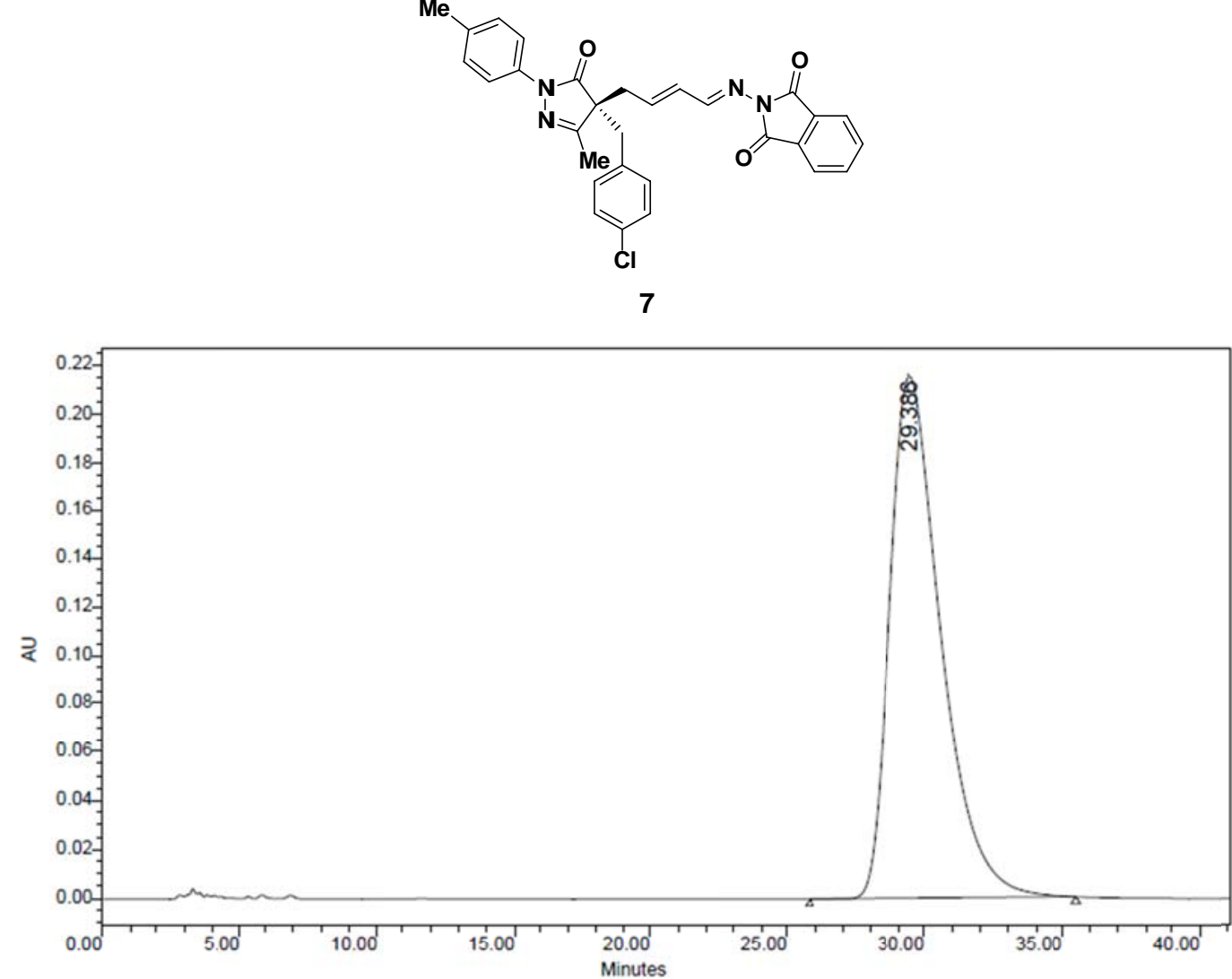

\begin{tabular}{|c|c|c|c|c|c|}
\hline & $\begin{array}{c}\text { RT } \\
(\mathrm{min})\end{array}$ & $\begin{array}{c}\text { Area } \\
\left(\mathrm{V}^{*} \mathrm{sec}\right)\end{array}$ & $\%$ Area & $\begin{array}{c}\text { Height } \\
(\mathrm{V})\end{array}$ & $\begin{array}{c}\% \\
\text { Height }\end{array}$ \\
\hline 1 & 29.386 & 27737486 & 100.00 & 215666 & 100.00 \\
\hline
\end{tabular}




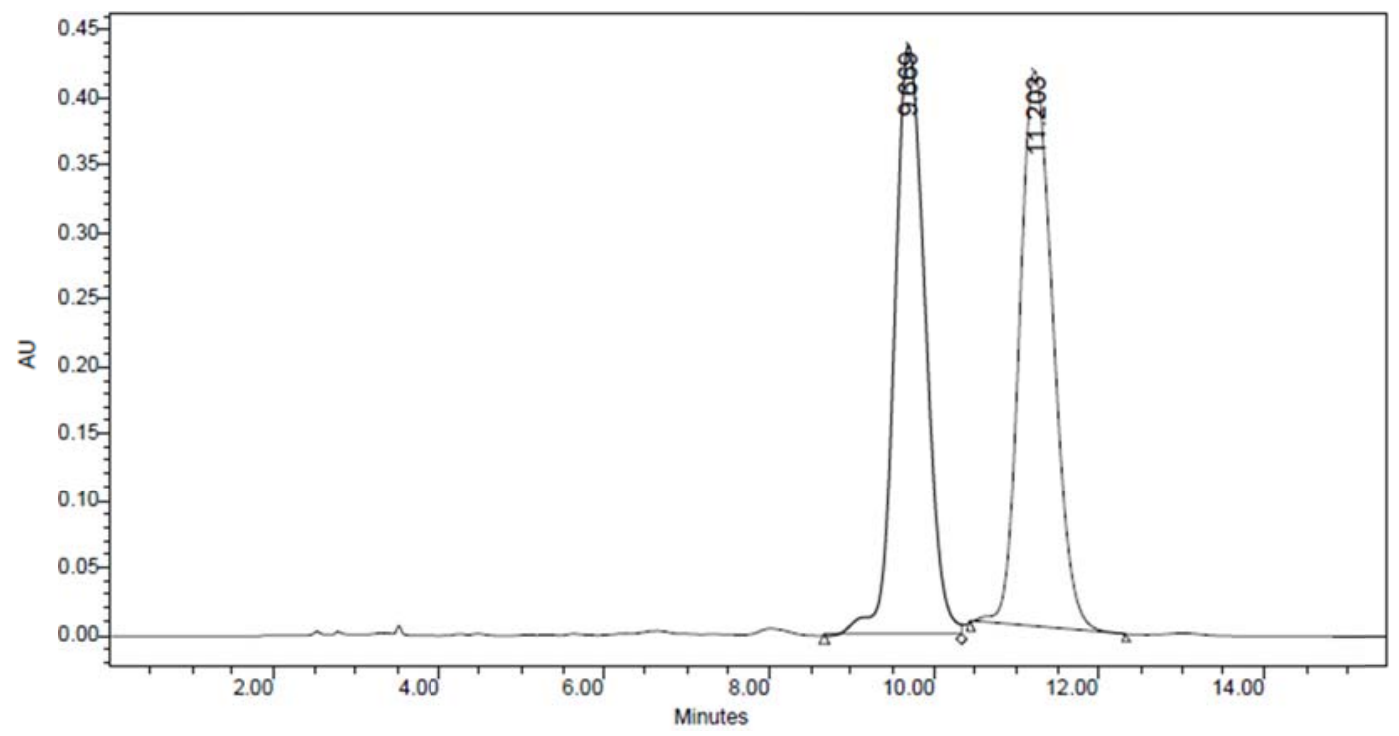

\begin{tabular}{|c|c|c|c|c|c|}
\hline & $\begin{array}{c}\text { RT } \\
(\mathrm{min})\end{array}$ & $\begin{array}{c}\text { Area } \\
\left(\mathrm{V}^{*} \mathrm{sec}\right)\end{array}$ & $\%$ Area & $\begin{array}{c}\text { Height } \\
(\mathrm{V})\end{array}$ & $\begin{array}{c}\% \\
\text { Height }\end{array}$ \\
\hline 1 & 9.669 & 11349802 & 49.58 & 439205 & 51.48 \\
\hline 2 & 11.203 & 11543167 & 50.42 & 414033 & 48.52 \\
\hline
\end{tabular}

Me

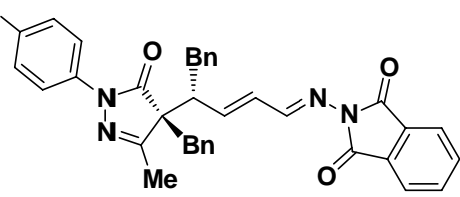

8

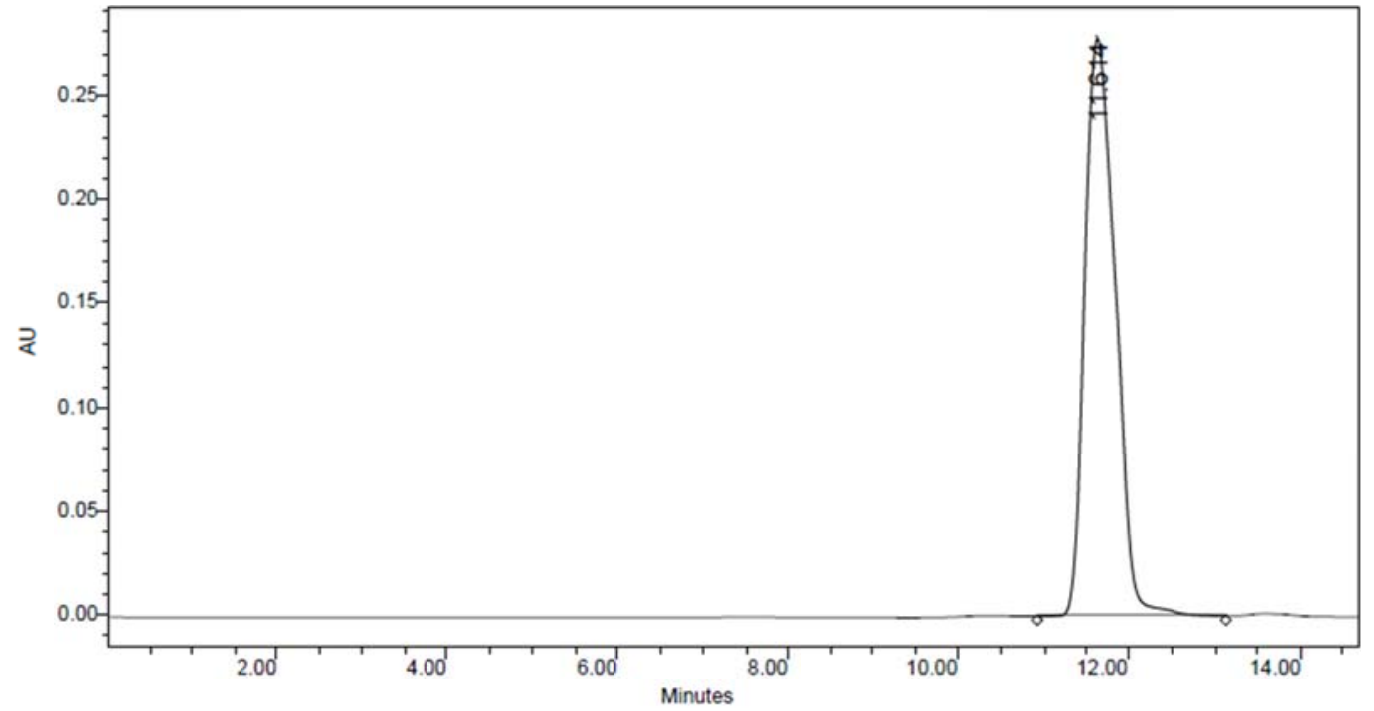

\begin{tabular}{|c|c|c|c|c|c|}
\hline & $\begin{array}{c}\text { RT } \\
(\mathrm{min})\end{array}$ & $\begin{array}{c}\text { Area } \\
\left(\mathrm{V}^{\prime} \mathrm{sec}\right)\end{array}$ & \% Area & $\begin{array}{c}\text { Height } \\
(\mathrm{V})\end{array}$ & $\begin{array}{c}\% \\
\text { Height }\end{array}$ \\
\hline 1 & 11.614 & 6959784 & 100.00 & 277872 & 100.00 \\
\hline
\end{tabular}




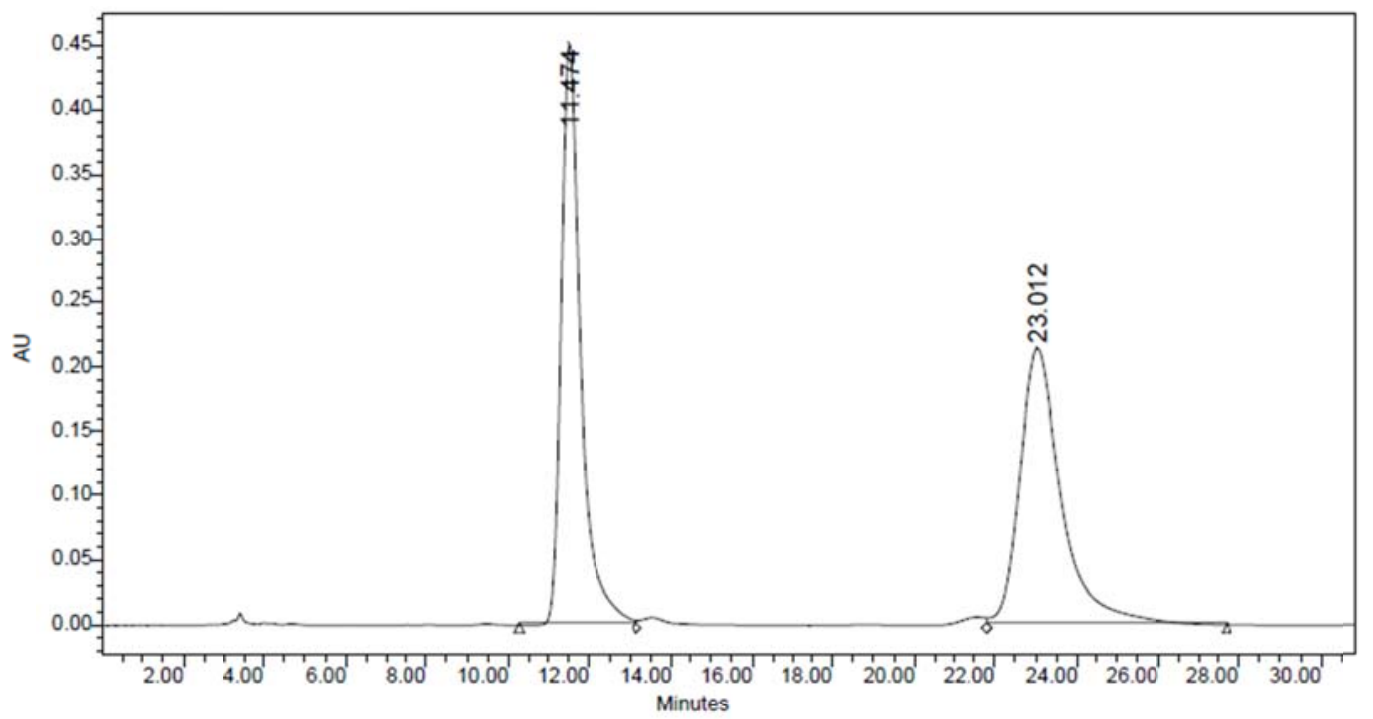

\begin{tabular}{|c|c|c|c|c|c|}
\hline & $\begin{array}{c}\mathrm{RT} \\
(\mathrm{min})\end{array}$ & $\begin{array}{c}\text { Area } \\
\left(\mathrm{V}^{*} \mathrm{sec}\right)\end{array}$ & $\%$ Area & $\begin{array}{c}\text { Height } \\
(\mathrm{V})\end{array}$ & $\begin{array}{c}\% \\
\text { Height }\end{array}$ \\
\hline 1 & 11.474 & 15196876 & 49.82 & 451452 & 67.77 \\
\hline 2 & 23.012 & 15305464 & 50.18 & 214684 & 32.23 \\
\hline
\end{tabular}

Me
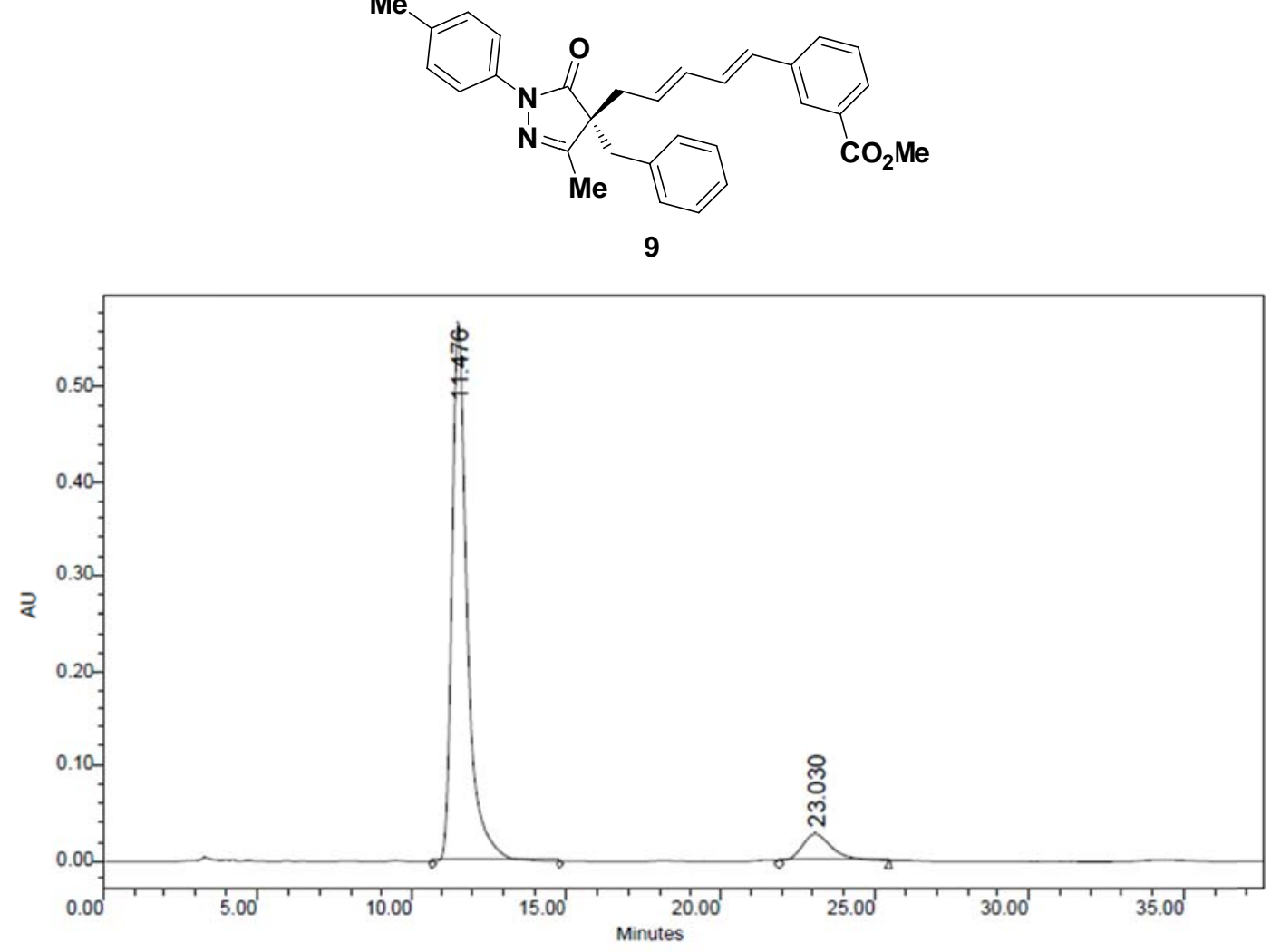

\begin{tabular}{|c|c|c|r|r|r|}
\hline & $\begin{array}{c}\mathrm{RT} \\
(\mathrm{min})\end{array}$ & $\begin{array}{c}\text { Area } \\
\left(\mathrm{V}^{*} \mathrm{sec}\right)\end{array}$ & \% Area & $\begin{array}{c}\text { Height } \\
(\mathrm{V})\end{array}$ & $\begin{array}{c}\% \\
\text { Height }\end{array}$ \\
\hline 1 & 11.476 & 19387368 & 90.94 & 568374 & 95.26 \\
\hline 2 & 23.030 & 1931543 & 9.06 & 28267 & 4.74 \\
\hline
\end{tabular}




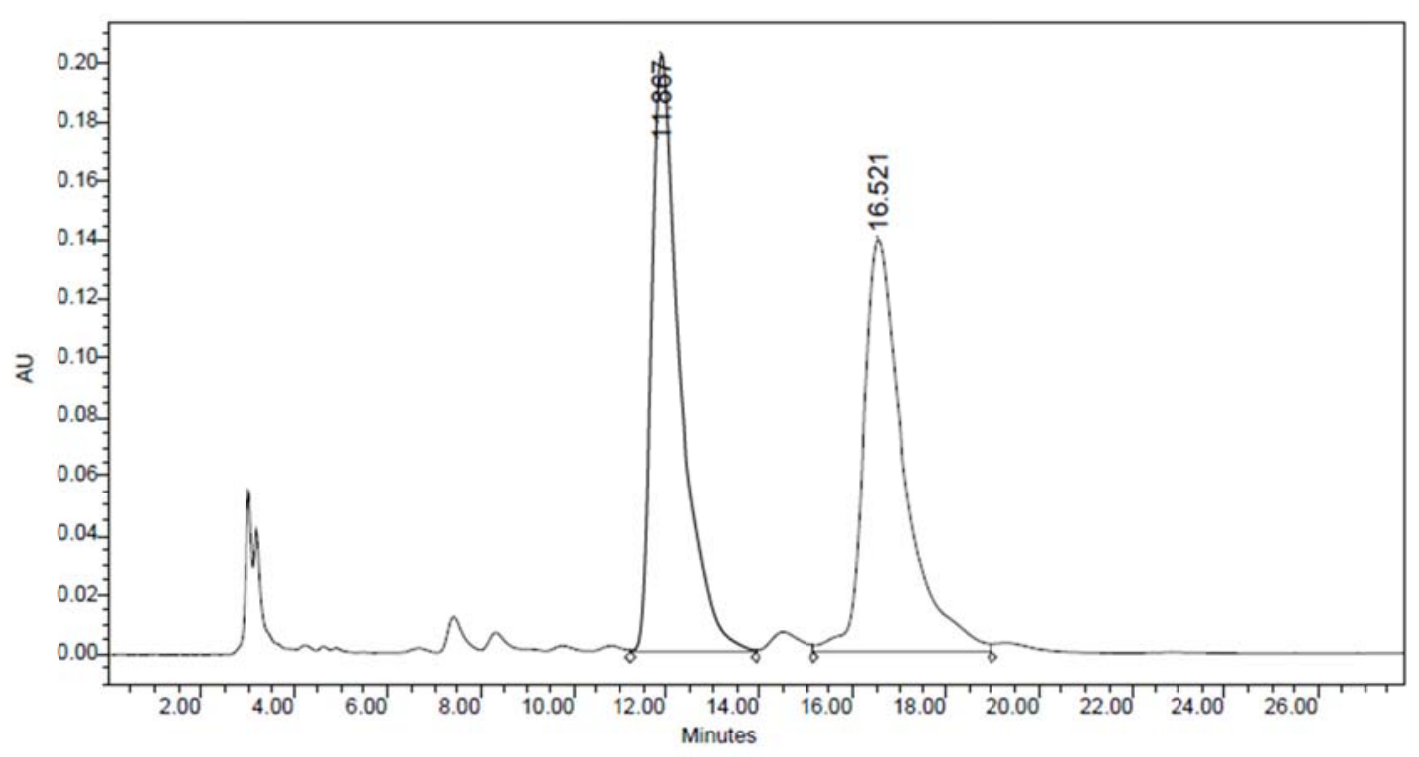

\begin{tabular}{|c|c|c|c|c|c|}
\hline & $\begin{array}{c}\mathrm{RT} \\
(\mathrm{min})\end{array}$ & $\begin{array}{c}\text { Area } \\
\left(\mathrm{V}^{*} \mathrm{sec}\right)\end{array}$ & $\%$ Area & $\begin{array}{c}\text { Height } \\
(\mathrm{V})\end{array}$ & $\begin{array}{c}\% \\
\text { Height }\end{array}$ \\
\hline 1 & 11.867 & 8770160 & 50.46 & 203371 & 59.11 \\
\hline 2 & 16.521 & 8610625 & 49.54 & 140659 & 40.89 \\
\hline
\end{tabular}
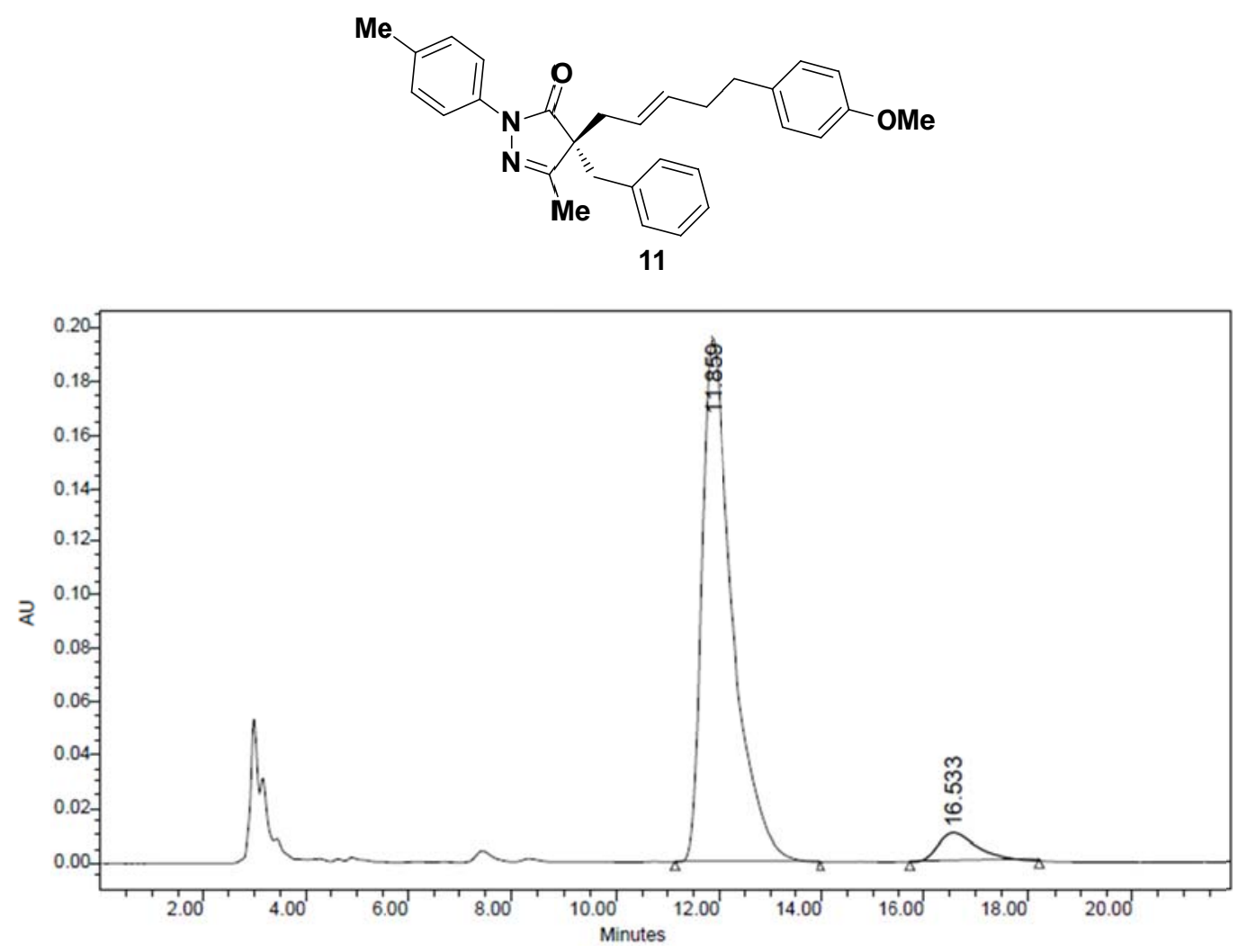

\begin{tabular}{|c|c|r|r|r|r|}
\hline & $\begin{array}{c}\text { RT } \\
(\mathrm{min})\end{array}$ & $\begin{array}{c}\text { Area } \\
\left(\mathrm{V}^{*} \mathrm{sec}\right)\end{array}$ & \% Area & $\begin{array}{c}\text { Height } \\
(\mathrm{V})\end{array}$ & $\begin{array}{c}\% \\
\text { Height }\end{array}$ \\
\hline 1 & 11.859 & 8061119 & 93.39 & 195896 & 94.85 \\
\hline 2 & 16.533 & 570213 & 6.61 & 10632 & 5.15 \\
\hline
\end{tabular}




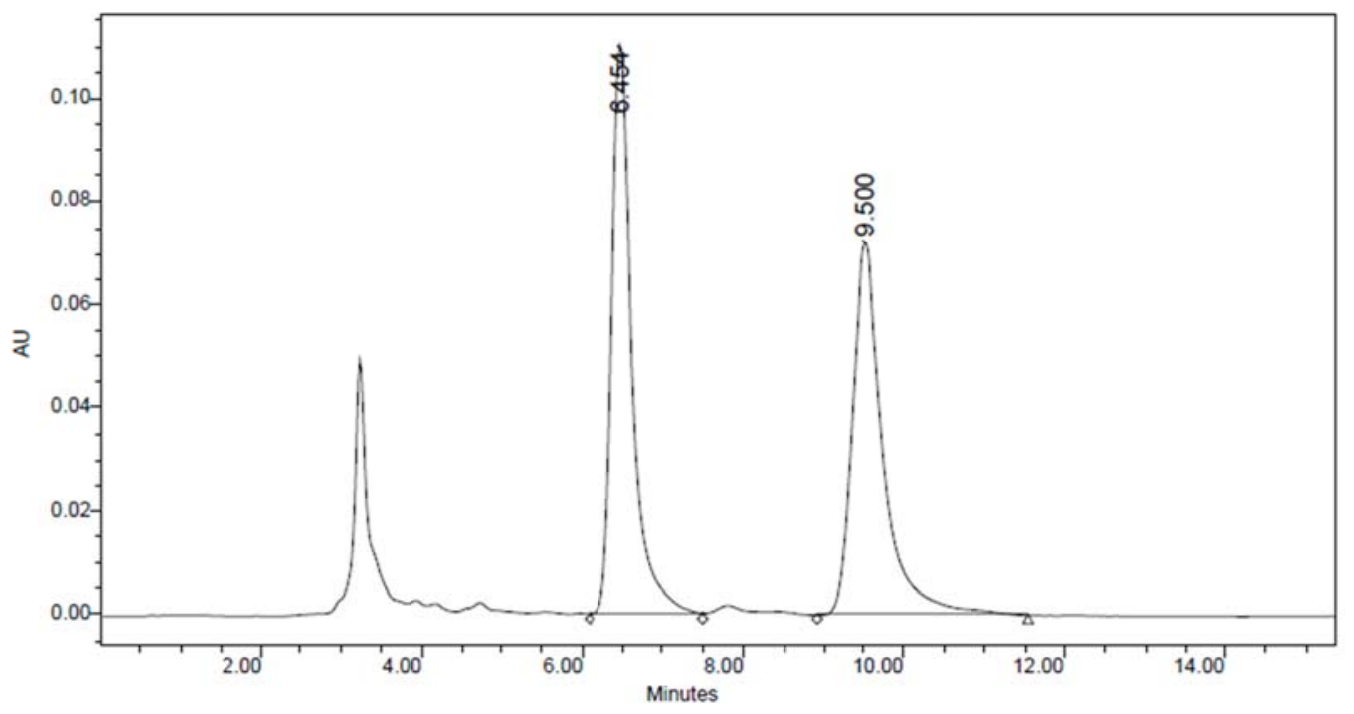

\begin{tabular}{|c|c|c|c|c|c|}
\hline & $\begin{array}{c}\text { RT } \\
(\mathrm{min})\end{array}$ & $\begin{array}{c}\text { Area } \\
(\mathrm{V} \text { *sec })\end{array}$ & $\%$ Area & $\begin{array}{c}\text { Height } \\
(\mathrm{V})\end{array}$ & $\begin{array}{c}\% \\
\text { Height }\end{array}$ \\
\hline 1 & 6.454 & 1966793 & 50.75 & 110611 & 60.48 \\
\hline 2 & 9.500 & 1908497 & 49.25 & 72283 & 39.52 \\
\hline
\end{tabular}

Me

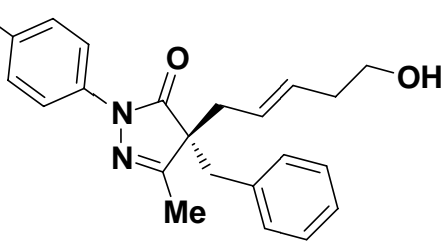

12

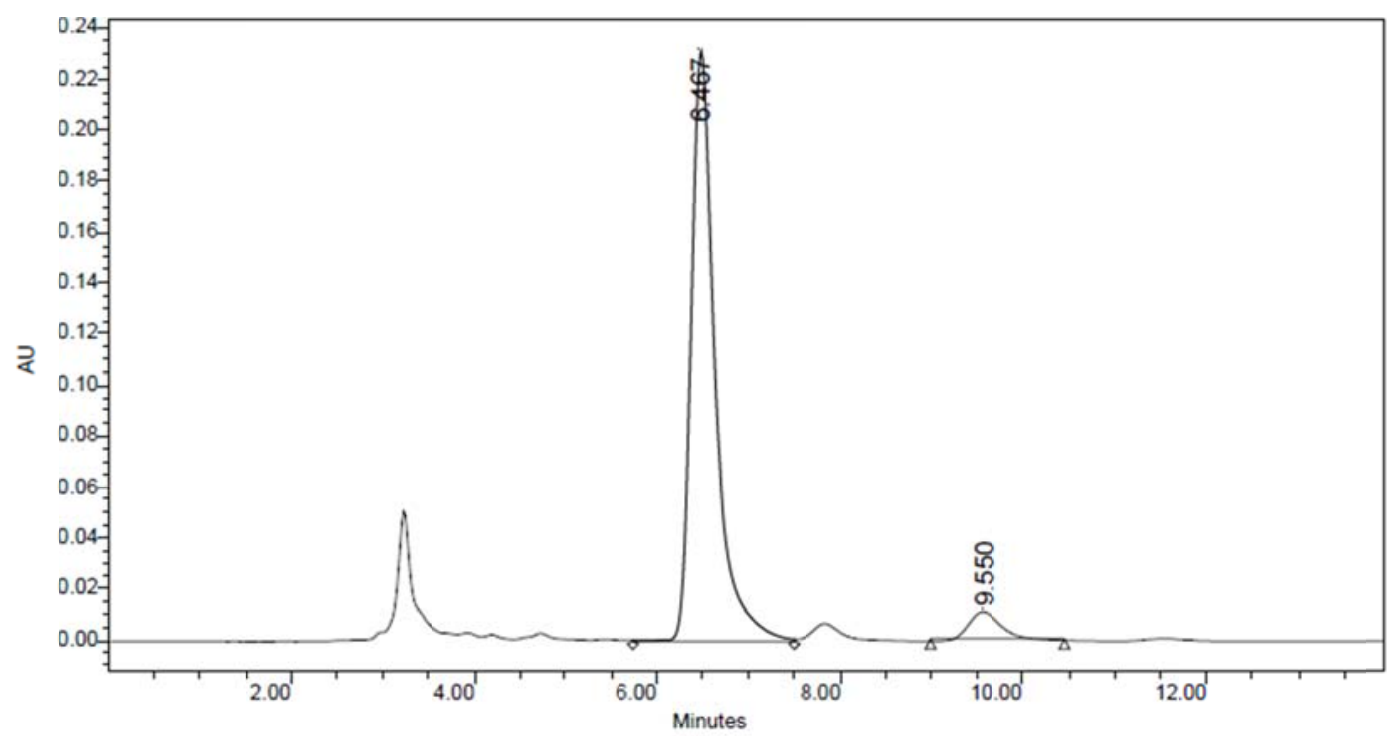

\begin{tabular}{|l|c|c|r|r|r|}
\hline & $\begin{array}{c}\mathrm{RT} \\
(\mathrm{min})\end{array}$ & $\begin{array}{c}\text { Area } \\
\left(\mathrm{V}^{*} \mathrm{sec}\right)\end{array}$ & $\%$ Area & $\begin{array}{c}\text { Height } \\
(\mathrm{V})\end{array}$ & $\begin{array}{c}\% \\
\text { Height }\end{array}$ \\
\hline 1 & 6.467 & 4193897 & 93.51 & 231748 & 95.35 \\
\hline 2 & 9.550 & 291258 & 6.49 & 11314 & 4.65 \\
\hline
\end{tabular}

